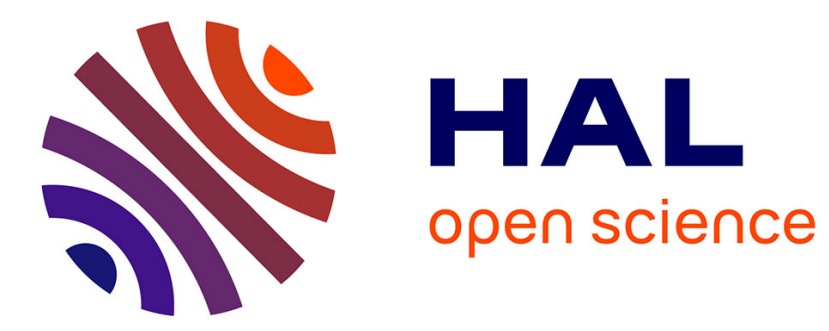

\title{
Poterie, métallurgie et verrerie au début du VIe s. à Gardanne (B.-du-Rh.)
}

Jean-Pierre Pelletier, Luc Poussel, Yves Rigoir, Jacqueline Rigoir, Lucy

Vallauri, Danièle Foy, Frédérique Audoin-Rouzeau, Françoise Brien-Poitevin

\section{- To cite this version:}

Jean-Pierre Pelletier, Luc Poussel, Yves Rigoir, Jacqueline Rigoir, Lucy Vallauri, et al.. Poterie, métallurgie et verrerie au début du VIe s. à Gardanne (B.-du-Rh.). Documents d'archéologie méridionale, 1991, 14, pp.277 - 350. 10.3406/dam.1991.1039 . halshs-01414501

\section{HAL Id: halshs-01414501 https://shs.hal.science/halshs-01414501}

Submitted on 12 Dec 2016

HAL is a multi-disciplinary open access archive for the deposit and dissemination of scientific research documents, whether they are published or not. The documents may come from teaching and research institutions in France or abroad, or from public or private research centers.
L'archive ouverte pluridisciplinaire HAL, est destinée au dépôt et à la diffusion de documents scientifiques de niveau recherche, publiés ou non, émanant des établissements d'enseignement et de recherche français ou étrangers, des laboratoires publics ou privés. 


\section{Poterie, métallurgie et verrerie au début du Vle s. à Gardanne} (B.-du-Rh.)

Jean-Pierre Pelletier, Luc Poussel, Yves Rigoir, Jacqueline Rigoir, Lucy Vallauri, Danièle Foy, Frédérique Audoin-Rouzeau, Françoise Brien-Poitevin

\section{Citer ce document / Cite this document :}

Pelletier Jean-Pierre, Poussel Luc, Rigoir Yves, Rigoir Jacqueline, Vallauri Lucy, Foy Danièle, Audoin-Rouzeau Frédérique, Brien-Poitevin Françoise. Poterie, métallurgie et verrerie au début du Vle s. à Gardanne (B.-du-Rh.). In: Documents d'Archéologie Méridionale, vol. 14, 1991. Le sanctuaire protohistorique de Roquepertuse. pp. 277-350; doi : 10.3406/dam.1991.1039

http://www.persee.fr/doc/dam_0184-1068_1991_num_14_1_1039

Document généré le 07/11/2016 


\title{
Résumé
}

Une vaste fosse-dépotoir a livré un échantillonnage varié de documents sur la vie de la localité durant l'Antiquité tardive. La vaisselle est représentée principalement par les Dérivées-desSigillées Paléochrétiennes et par un lot important de verrerie; les ustensiles culinaires le sont par les céramiques communes grises. Ossements et coquillages témoignent d'une alimentation variée. Des creusets et des moules de fonderie attestent l'existence d'ateliers où étaient travaillés le verre, le fer et le bronze. Dans ce dernier métal étaient produits des objets luxueux comme des éléments de pieds de lit ou de chandelier. Certaines caractéristiques des poteries font supposer une fabrication de proximité. Ces artisanats suffisaient à la clientèle locale : les importations (d'Afrique du Nord ou d'Italie), généralement bien représentées à cette époque, sont ici pratiquement absentes. Poteries et verres sont ornés de chrismes et de croix.

\begin{abstract}
A large garbage-pit has yielde varied samples of documents on the life of the locality during late Antiquity. Crockery is mainly represented by Sigillata-derived Early Christian pottery and a large batch of glassware ; kitchen ustensils by common gray pottery. Bones and shells bear witness to a varied diet. Crucibles and foundry moulds prove the existence of workshops where glass, iron and bronze were processed. The latter metal was used for luxury objects like legs for beds and bases for candelsticks. Some characteristics of the pottery suggest manufacture in the neighbourhood. This industry met the requirements of the local customers : imported goods (from North Africa or Italy), usually well represented in this period, are practically absent here. Pottery and glassware are decorated with the monogram of Christ and with crosses.
\end{abstract}




\title{
Poterie, métallurgie et verrerie au début du VI' s. à Gardanne (B.-du-Rh.)
}

\author{
par \\ Jean-Pierre PELLETIER *, Luc POUSSEL **, Yves et Jacqueline RIGOIR *, \\ Lucy VALLAURI * et Danièle FOY * \\ avec les contributions de Frédérique AUDOIN-ROUZEAU ** et Françoise BRIEN-POITEVIN ****
}

Résumé - Une vaste fosse-dépotoir a livré un échantillonnage varié de documents sur la vie de la localité durant l'Antiquité tardive. La vaisselle est représentée principalement par les Dérivées-des-Sigillées Paléochrétiennes et par un lot important de verrerie ; les ustensiles culinaires le sont par les céramiques communes grises. Ossements et coquillages témoignent d'une alimentation variée. Des creusets et des moules de fonderie attestent l'existence d'ateliers où étaient travaillés le verre, le fer et le bronze. Dans ce dernier métal étaient produits des objets luxueux comme des éléments de pieds de lit ou de chandelier. Certaines caractéristiques des poteries font supposer une fabrication de proximité. Ces artisanats suffisaient à la clientèle locale : les importations (d'Afrique du Nord ou d'Italie), généralement bien représentées à cette époque, sont ici pratiquement absentes. Poteries et verres sont ornés de chrismes et de croix.

Mots-clés : Céramique commune grise, Dérivées-des-Sigillées Paléochrétiennes, Verre, Métallurgie, Faune, Coquillages, Christianisme, Antiquité tardive, Gardanne, Bouches-du-Rhône.

Abstract - A large garbage-pit bas yielde varied samples of documents on the life of the locality during late Antiquity. Crockery is mainly represented by Sigillata-derived Early Christian pottery and a large batch of glassware; kitchen ustensils by common gray pottery. Bones and shells bear witness to a varied diet. Crucibles and foundry moulds prove the existence of workshops where glass, iron and bronze were processed. The latter metal was used for luxury objects like legs for beds and bases for candelsticks. Some characteristics of the pottery suggest manufacture in the neighbourbood. This industry met the requirements of the local customers : imported goods (from North Africa or Italy), usually well represented in this period, are practically absent bere. Pottery and glassware are decorated with the monogram of Christ and with crosses.

Keywords : Common gray pottery, Sigillata-derived Early Christian pottery, Glassware, Metallurgy, Fauna, Shells, Christianity, Late Antiquity, Gardanne, Bouches-du-Rhône. 


\section{Le site, la découverte}

A dix km au sud-est d'Aix-en-Provence et à un $\mathrm{km}$ à l'est du gros bourg de Gardanne, le quartier Notre-Dame est situé en bordure de plaine, à proximité du ruisseau de Saint-Pierre (fig. 1).

L'occupation antique, signalée par l'abbé M. Chaillan au début du siècle (Chaillan 1910, 7), a été confirmée par des prospections de la Société Archéologique Méditerranéenne [S.A.M.] depuis

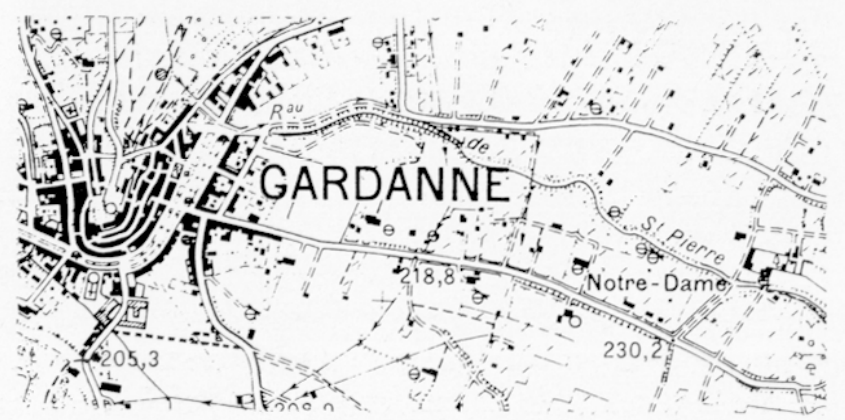

1 Situation du quartier Notre-Dame. Carte I.G.N. Aix $n^{\circ} 5$ au $1 / 20000$.

12 Quartier Notre-Dame : plan de situation de la fosse et des vestiges. $1:$ zone avec constructions antiques entrevues (murs, stuc...). 2 : zone avec constructions en grand appareil entrevues. 3 : sépultures sur deux niveaux constatées dans une tranchée. 4 : sépultures "médiévales" en surface. 5 : Château Pitty (école).

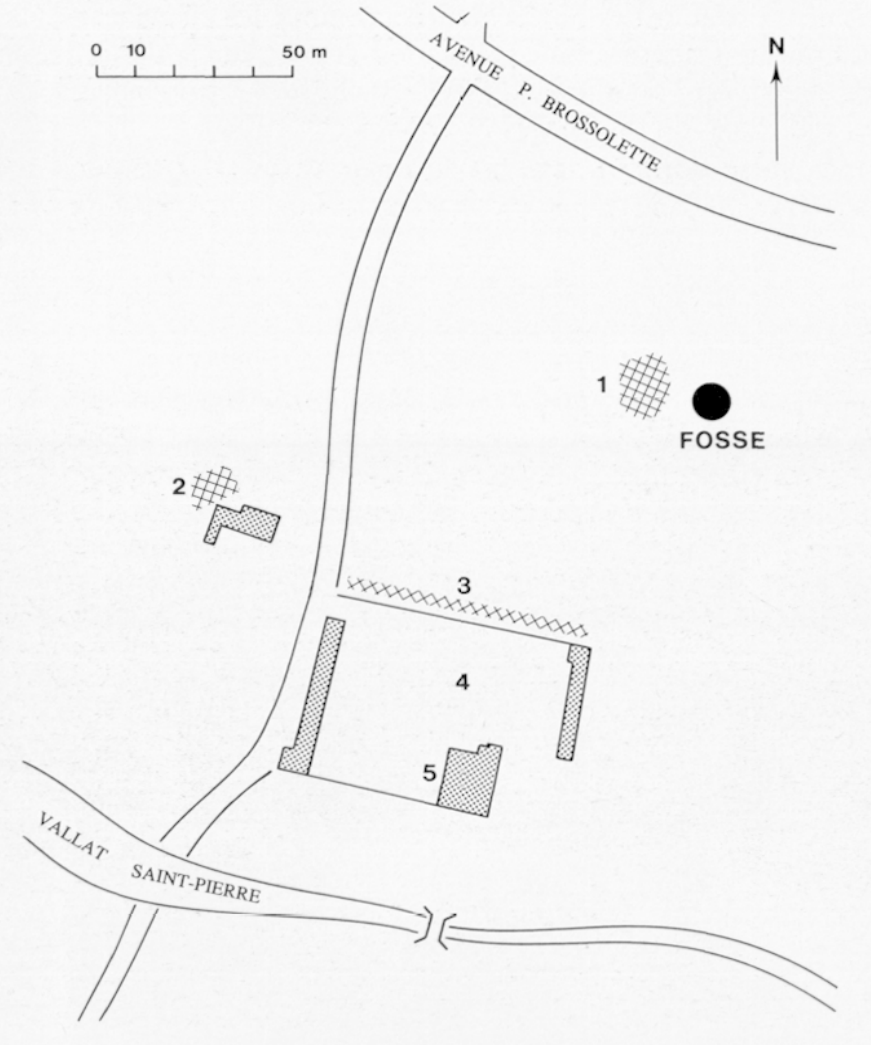

1975 (ramassages de tessons de céramiques campaniennes, sud-gauloises, Dérivées-des-Sigillées Paléochrétiennes [DS.P.], communes grises, d'amphores...). L'implantation d'une Z.A.C. en 1980 a donné lieu à de très importants terrassements, au cours desquels des traces de constructions furent entrevues, ainsi que des sépultures attribuables à une nécropole de l'Antiquité tardive et du Moyen Age (fig. 2).

\subsection{L'EGLISE NOTRE-DAME}

Le toponyme cadastral Notre-Dame correspond au vocable de l'église paroissiale mentionnée en tant que telle dans le cartulaire de l'abbaye de Saint-Victor à la fin du XI ${ }^{e}$ s. (Guérard 1857, chartes 222 de 1093 et 224 de 1098). M. Chaillan avait remarqué que des restes du monument se trouvaient encore dans la "campagne" de M. F. Baret (Chaillan 1910, 51 ; Marco 1989, 34-36), qui est une bastide du $\mathrm{XVIII}^{\mathrm{e}}$ s. aujourd'hui connue sous le nom de Château Pitty et devenue école communale (fig. $2, \mathrm{n}^{\circ} 5$ ).

Dans le bâtiment récemment restauré et aménagé pour cette destination pédagogique, trois supports sont encore observables, selon un alignement nordsud. Le plus méridional est constitué de blocs de grand appareil marqués d'une rainure verticale médiane ; un trou de louve est apparent. Le support médian est constitué d'une colonne reposant sur une base antique en réemploi. Au nord, un pilastre appareillé fait penser à un support médiéval adossé. Une arcature en plein cintre, visible dans un placard à l'étage, réunit le support méridional au support médian, sur lequel il prend appui par une imposte à mouluration complexe. Dans le cas normal d'une orientation de l'église à laquelle semblent appartenir ces vestiges, la disposition de ces trois supports impliquerait un lieu de culte formé de deux nefs larges de 3,30 m environ. Selon M. Fixot, un tel type de plan à deux nefs n'est pas exceptionnel en Provence (Codou 1989 et 1991). En outre, il ne serait pas impossible que le bras nord du transept subsiste dans la saillie de l'angle nord-est du bâtiment. Une tombe orientée a été découverte dans la chaufferie, à $2 \mathrm{~m}$ à l'ouest des supports médiévaux (1). D'autres sépultures orientées, en "fosses recouvertes de pierres", ont été observées dans la cour de l'école (fig. $2, \mathrm{n}^{\circ} 4$ ) et dans les coupes d'une tranchée où était visible un deuxième niveau plus profond de tombes orientées, formées de coffrages en tegulae disposées en bâtière ou posées à plat sur les corps (fig. $2, \mathrm{n}^{\circ} 3$ ).

De nombreux lieux de culte sont mentionnés sur le territoire de Gardanne au XIe s., mais l'église Sainte-Marie est qualifiée de paroisse. Ainsi, l'occupation du quartier Notre-Dame pourrait refléter un système paroissial ancien (antérieur au castrum), implanté 


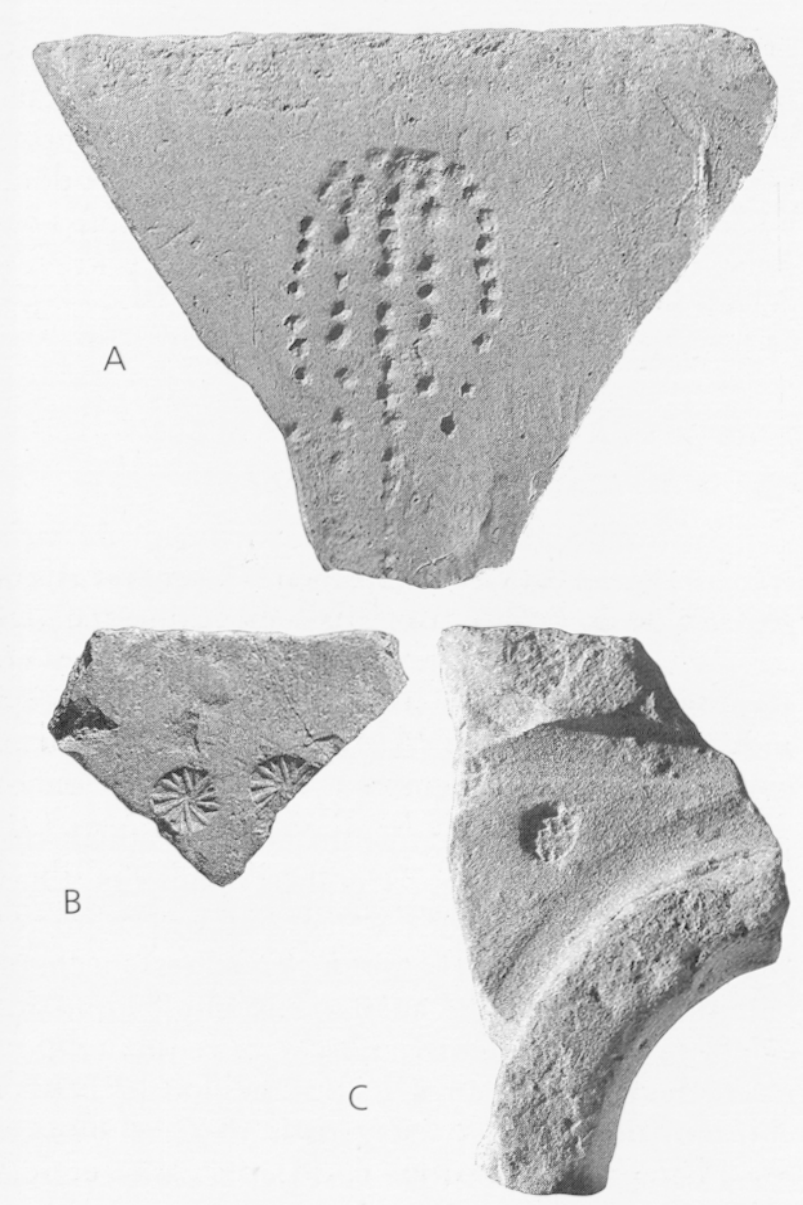

13 Briques et tuile trouvées à proximité de la fosse. A : fragment de brique avec empreinte de semelle cloutée. B : fragment de brique décoré de rouelles imprimées. $C$ : fragment de tuile façonnée avec un orifice circulaire et décorée de nombreuses empreintes de rouelles [éch. 1/3].

14 La fosse en cours de dégagement. tivel, que se fixa l'agglomération. La paroisse y fut transférée dans le courant du Moyen Age aux dépens de l'ancienne église du quartier Notre-Dame, où le cadastre du XIX ${ }^{e}$ s. ne montre que quelques bâtisses isolées dans la campagne autour de la bastide.

\subsection{LA FOSSE-DEPOTOIR}

Lors de la construction de la Z.A.C. en 1980, les travaux de terrassement exécutés très rapidement par les promoteurs ont empêché toute recherche méthodique. Divers ramassages ont pu être effectués à quelques dizaines de mètres plus au nord. Ils ont fourni notamment de la sigillée claire africaine, de la luisante, de la DS.P. (fig. $8 ; 9 ; 31 ; 32$ ) et divers fragments de briques et de tuiles (fig. 3).

La seule intervention de sauvetage archéologique a pu être conduite pendant un week-end, en l'absence des ouvriers, sur un tronçon de fosse mis en évidence le vendredi et qui fut vidé de son contenu et rempli de béton le lundi suivant. Cette fosse, creusée dans le substrat argileux, était profonde de $2 \mathrm{~m}$ pour une largeur de $4 \mathrm{~m}$ (fig. 2 et 4). En bordure, l'emplacement d'une tombe était encore matérialisé par la présence de tegulae; le squelette, toujours en connexion anatomique (fig. 5), avait glissé un mètre plus bas, dans la fosse, peu après le creusement de celle-ci. Au-dessus du crâne, une des tuiles de couverture, la seule décorée, semblait avoir été posée après le glissement. Il est peu fréquent de retrouver un décor aussi soigné sur une tegula; pourtant un fragment analogue a été recueilli lors de prospections par la S.A.M. au col Sainte-Anne, à $5 \mathrm{~km}$ au sud, sur la commune de Simiane-Collongue.

Le comblement de la fosse était constitué d' un sédiment sablonneux gris foncé contenant de nom-

auprès d'une des villae d'origine antique. D'autres vestiges antiques ont été signalés au quartier Saint-Pierre, près du centre actuel du village (Statistique des B.-d.Rh. 1824, 894 ; Chaillan 1910, 7 ; Benoît 1934, 42, $n^{\circ}$ 106-107). En 1022, l'église Saint-Pierre, qui devient alors prieuré de l'abbaye de Saint-Victor, est déjà située non loin du castrum (Guérard 1857, charte 257), et à la fin du $\mathrm{XI}^{\mathrm{e}}$ s. les églises et chapelles des saints Michel, Pierre, Valentin et Baudile semblent composer un même groupe, bien distinct de la paroissiale Sainte-Marie. C'est près de ce pôle, la colline du Cap-

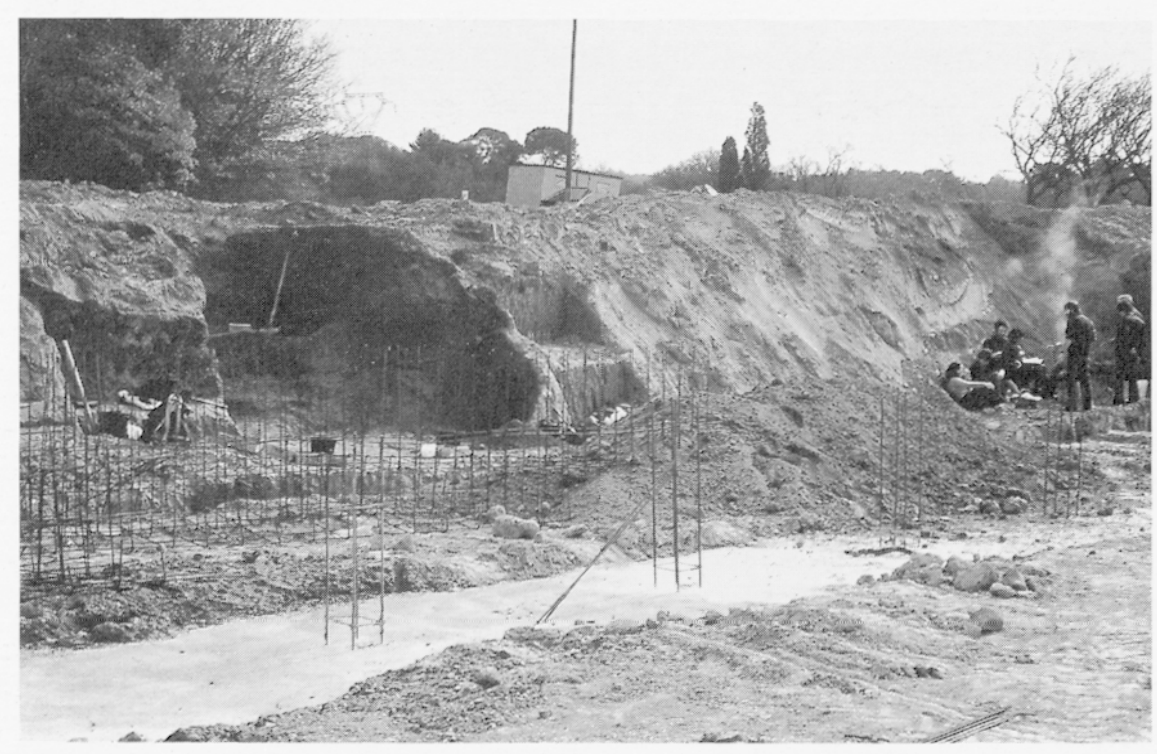



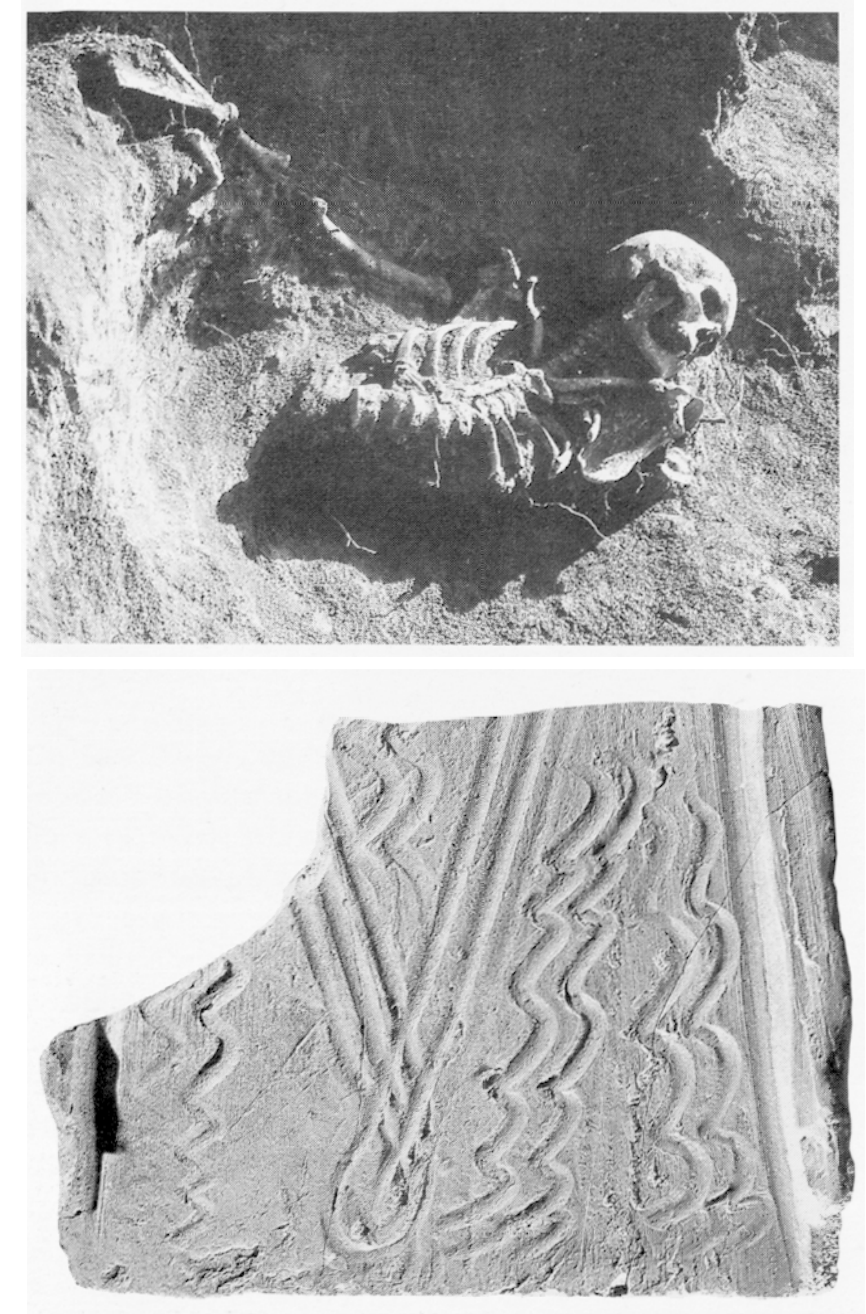

15 Le squelette dans la fosse et la tuile de couverture décorée [éch. 1/3].

breux tessons de poteries, verres, creusets et moules de bronziers, ossements animaux, coquillages, fragments métalliques et débris divers. Le matériel a été récupéré en partie par la fouille rapide, et en partie par le tamisage des terres restantes, "expurgées" par les pelles mécaniques et mises à disposition en bordure du chantier avant le bétonnage. Aussi ne peuton considérer être en présence que d'une fraction des informations concernant ce dépotoir, proche d'un habitat qui n'a pu être reconnu. Toutefois, malgré ces lacunes, la quantité et la qualité des éléments recueillis justifient une étude détaillée qui n'apparaissait pas évidente ou nécessaire au premier abord. Une présentation partielle des céramiques par Michel Piskorz lors d'une réunion de la C.A.T.H.M.A. en 1987 avait éveillé notre curiosité, dans le cadre des recherches entreprises sur les DS.P. et les communes grises, les pièces de comparaison étant encore peu nombreuses. L'examen de l'ensemble du matériel dans le dépôt de la S.A.M. confirma la richesse et la diversité des découvertes, et entrainna cette publication préparée au Laboratoire d'Archéologie Médiévale Méditerranéenne.

\section{Le mobilier céramique}

\subsection{LES FREQUENCES RELATIVES}

Au total 5269 tessons se répartissent de façon très inégale : 185 “indéterminés" (éclats d'amphores, céramiques communes résiduelles diverses, etc.), 4 sigillées claires importées, 673 DS.P. et 4407 communes grises. Pour les 5084 tessons identifiés, la sigillée claire représente $0,078 \%$, les DS.P. $13,23 \%$, et la commune grise $86,68 \%$.

$\mathrm{La}$ fosse présente ainsi un faciès original, bien différent de ce qui a pu être observé ailleurs. Outre l'absence presque totale de céramiques importées (sigillées africaines ou communes méditerranéennes), les amphores sont elles aussi inexistantes, à quelques éclats près, non identifiables. Conséquence de la rareté des sigillées claires, les poteries locales DS.P. et communes grises se partagent le matériel de cette fosse dans les proportions de 13 et $87 \%$ (comptage par tessons). Plusieurs points autorisent l'établissement de rapports entre ces deux productions pourtant très différentes à première vue ; ils feront l'objet de paragraphes communs.

\subsection{LA FRAGMENTATION}

Dans l'étude de Saint-Blaise (2), il avait été proposé pour les trois catégories de vaisselle un "coefficient de fragmentation" établi d'après le rapport entre le nombre de tessons et le nombre de "vases vrais" (ou N.M.I.). Il était de 10,63 pour les DS.P. et de 33,6 pour les céramiques communes grises. $\mathrm{Ce}$ calcul a été reproduit ici avec les chiffres de 673 tessons et de 191 vases pour les premières, de 4407 tessons et de 425 vases pour les secondes. Les résultats obtenus : 3,5 pour les DS.P. et 10,37 pour les communes, sont très éloignés de ceux de Saint-Blaise (tabl. I et fig. 6).

Cependant, si l'on compare non plus de site à site, où il est évident que les conditions de fragmentation et de dispersion peuvent être différentes, mais de catégorie à catégorie, on observe un rapport constant de 1 à 3 , et ce malgré les nuances évoquées ci-dessus. Très peu de recollages ont pu être effectués sur les DS.P., alors qu'ils sont apparus particulièrement nombreux sur les communes : outre une quin- 


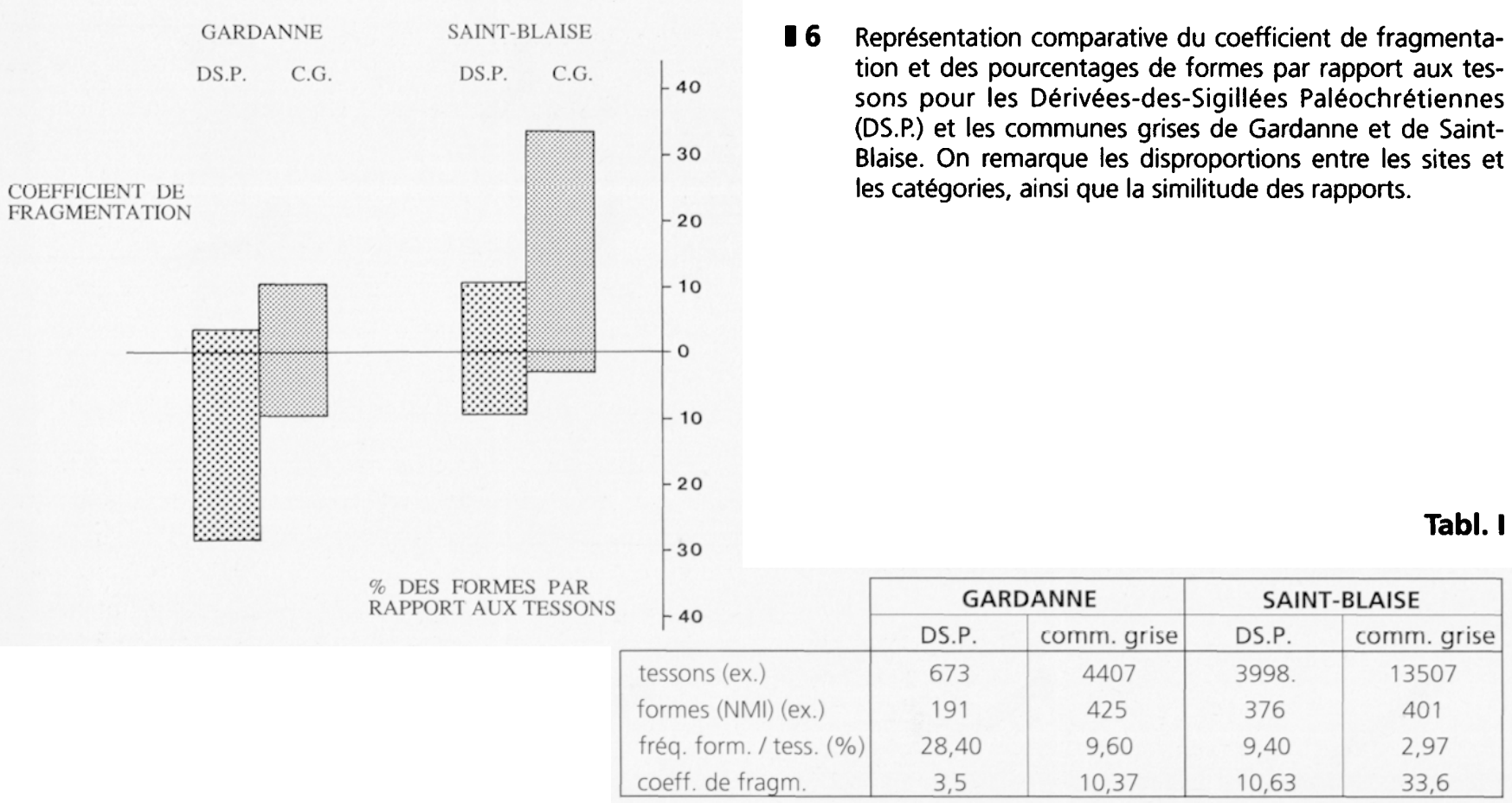

zaine de formes pour lesquelles les profils sont complets après recollage, plusieurs dizaines d'objets sont largement identifiables ainsi qu'en témoignent les planches de dessins. Ce résultat a pu être obtenu par la dimension plus importante des tessons et la nature $\mathrm{du}$ contexte, un dépotoir homogène bien différent de niveaux successifs d'occupation en habitat.

La nature des poteries influe elle aussi sur la fragmentation. Dans les DS.P., il y a peu de tessons de panses ou de tessons non décorés pour lesquels on utilise le terme "informe" : cela tient au fait qu'il y a peu de cruches, pratiquement jamais d'urnes, mais au contraire des formes basses et peu pansues. Alors que dans les céramiques communes, les tessons de panse sont toujours très nombreux, isolés ou sur un même objet reconstitué en tout ou partie ; en outre, l'absence de décors tend elle aussi à faire diminuer le nombre d'objets identifiables.

\subsection{LES CERAMIQUES SIGILLEES CLAIRES IMPORTEES}

Les céramiques sigillées claires importées (fig. 7 et 8) sont presque absentes de la fosse : 4 tessons au total ont été retrouvés et appartiennent seulement à trois formes.

- La céramique sigillée claire africaine est représentée par un fragment de lampe dont seul le bec brûlé a été conservé, ce qui ne permet aucune identification de type en l'absence du décor et de la queue de préhension.

Un fragment de plat rectangulaire de même origine est remarquable par son riche décor moulé. Sur le marli d'une forme Hayes 56, en pâte D1-D2, on observe une panthère chargeant à gauche et derrière elle un sanglier marchant à droite. Ce tesson exceptionnel pour notre région a fait l'objet d'une étude particulière par R. Guéry (1990, 266-271); il se rattache à des modèles fréquents en Afrique du Nord du dernier quart du $\mathrm{IV}^{e}$ à la première moitié du $\mathrm{V}^{e} \mathrm{~s}$. à Carthage (Salomonson 1969, pl. IV 1, sur pâte C4 ; Guéry 1972, 121, fig. 24, sur pâte D1).

- Un rebord de céramique sigillée phocéenne tardive (fig. 7), de forme Hayes 3, fournit un terminus a quo pour le comblement de la fosse : cette forme est attestée dans le midi de la France dès le milieu du V' s. (C.A.T.H.M.A. 1986, 36-38).

Un ramassage effectué autour de la fosse a permis de récolter 28 tessons de céramique sigillée africaine, en pâtes DI$\mathrm{D} 2$ et $\mathrm{C5}$, dont la plupart se rattachent à la seconde génération des productions tunisiennes (Hayes 1972 ; Atlante 1981 ; C.A.T.H.M.A. 1986, 36-39; Démians d'Archimbaud et al. à paraître, fig. 43 à 66). Cette observation plaiderait en faveur d'une datation plutôt tardive pour l'habitat environnant.

Inventaire (fig. 8):

$\mathrm{N}^{\circ} 2$ : Hayes 59A; 3 : Hayes 61, type Lamboglia 54, Atlante, tav. XXXV n 3 ; 1 et 4 : Hayes 67, type Lamboglia 42 Vintimiglia, Atlante, tav. XXXVIII $n^{\circ} 3 ; 5$ : Hayes $81 ; 6$ : Hayes $84 ; 7:$ Hayes 82 (deux exemplaires) ; 8 : Hayes 87A

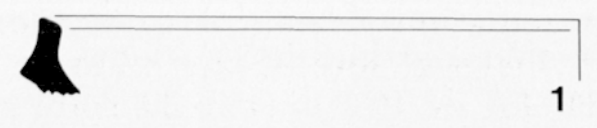

17 Sigillées Claires trouvées dans la fosse : sigillée phocéenne tardive forme Hayes 3 (le plat moulé $H$. 56 est figuré dans Guéry 1990, 268, fig. 6) [éch. 1/3]. 

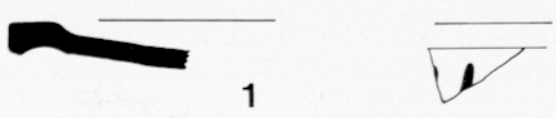

2
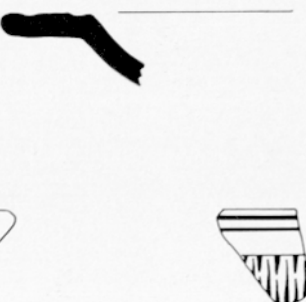

5
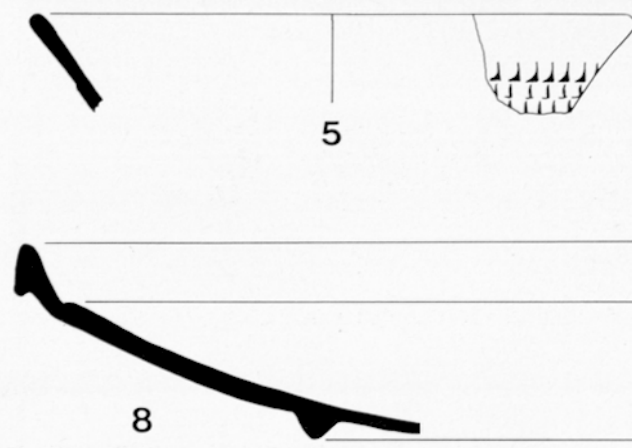

8

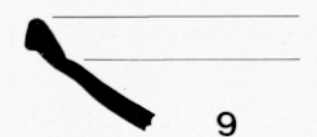

9

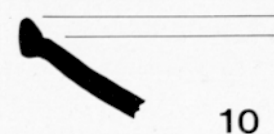

10

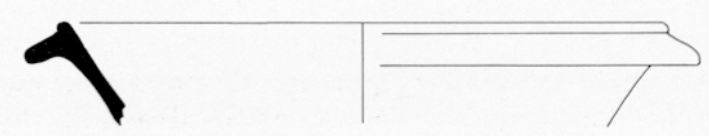

13

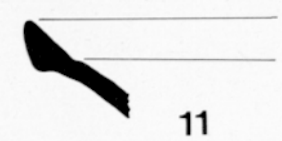

11
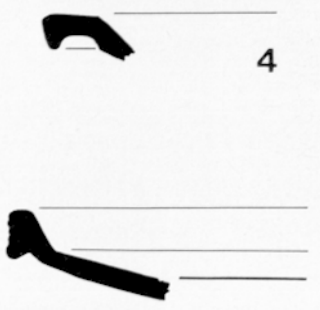

7

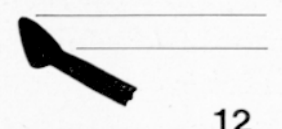

12

18 Sigillées Claires africaines trouvées autour de la fosse. $\mathrm{N}^{\circ} 1:$ plat moulé rectangulaire ; $2: \mathrm{H} .59 \mathrm{~A}($ diam. $: 35 \mathrm{~cm}) ; 3: \mathrm{H} .61$ (diam. : $38 \mathrm{~cm}$ ) ; $4:$ H. 67 (diam. : $40 \mathrm{~cm}$ ) ; $5:$ H. $81 ; 6:$ H. $84 ; 7:$ H. $82 ; 8:$ H. 87 A $; 9:$ H. 87 B ; 10 et $11:$ H. $87 / 61 / 104$ ?: $12:$ H. 61 variante tardive; $13:$ H. $91 \mathrm{C} ; 14:$ H. $12 ; 15:$ Martin NV IV [éch. 1/3].

(quatre ex.) ; $9:$ Hayes 87B ; 10 et $11:$ Hayes 87-61-104 ? (deux ex.); 12 : Hayes 61 variante tardive, Atlante, tav. XXXV $n^{\circ} 6 ; 13$ : Hayes $91 \mathrm{C} ; 14$ : Hayes $12 \mathrm{n}^{\circ} 1$, Atlante, tav. III $n^{\circ} 11 ; 15$ : Martin NV IV, Hayes 93 ; un rebord de plat rectangulaire moulé de forme inédite (Guéry 1990, 268, fig. $1 \mathrm{n}^{\circ} 3$ ).

\subsection{LES CERAMIQUES DERIVEES- DES-SIGILLEES PALEOCHRETIENNES}

\subsubsection{Formes}

Les 673 tessons de cette catégorie représentent un ensemble constitué de 31 assiettes ( $23 \%), 11$ coupelles $(8,2 \%), 60$ bols $(44,8 \%), 23$ vases fermés $(17 \%)$ et 9 mortiers, soit $76 \%$ de vaisselle de table et $24 \%$ d'ustensiles culinaires.

Dans le détail, on peut compter 12 formes 1,7 formes 3, 13 formes 18, 9 formes 29 et 4 formes 63 .
Ce catalogue est fragmentaire : de nombreuses variantes, quelquefois étapes intermédiaires entre les formes types, n'ont pas encore été répertoriées. On constate d'après cet inventaire que la typologie est loin d'être close.

Les assiettes se partagent à peu près également entre assiettes creuses et assiettes plates. Celles-ci, de grand diamètre, sont probablement des plats collectifs de présentation d'aliments solides. Les premières étaient plutôt des récipients individuels où pouvaient être servis des mets liquides ou pâteux, soupes ou purées, dont l'existence est attestée par la présence des mortiers. Plus exactement appelés en allemand "Reibschalen", ancêtres des moulinettes et des mixeurs, nombreux depuis le Haut Empire, leur surface intérieure rendue rugueuse par des guillochis dans la forme 91 de sigillee claire $D$ l'est encore plus efficacement en DS.P. et en commune par des inclusions, le plus fréquemment de fragments de basalte. La présence systématique de becs verseurs sur leur 

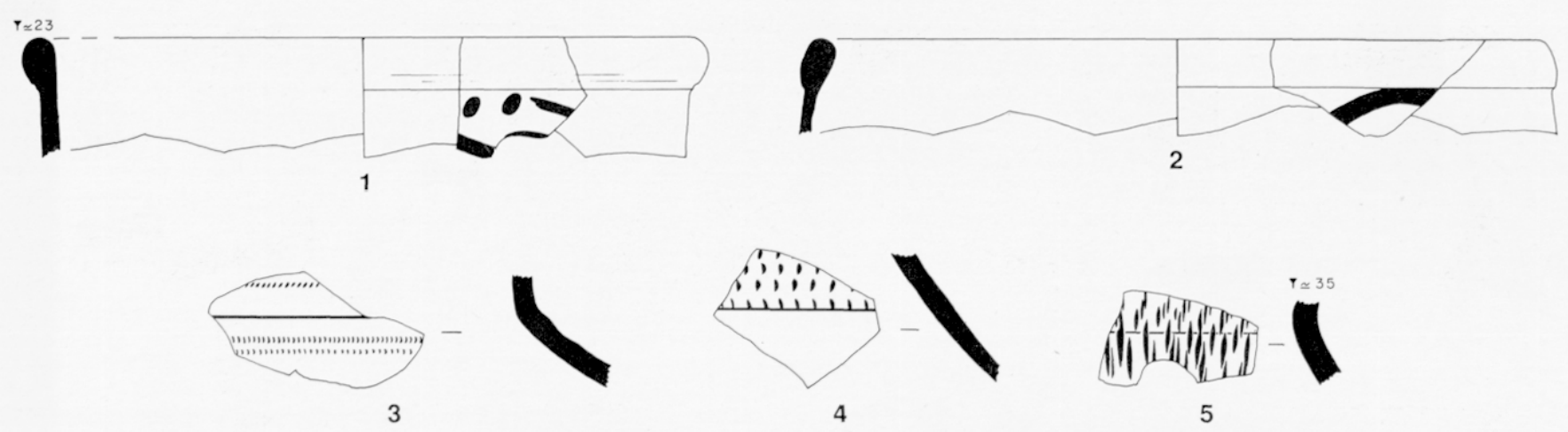

19 Luisante trouvée autour de la fosse. $N^{\circ} 1$ et $2:$ bords de forme Lamboglia $1 / 3$ décorés de peinture blanche; 3 et $4:$ fragments de panses de la même forme ; 5 : fragment de panse, forme Lamb. 2 [éch. 1/3].

rebord témoigne de la fluidité des farines ou brouets obtenus. La collerette ou "listel" a pu servir à caler ces ustensiles, peut-être sur un autre récipient. Dans ce cas, la possibilité d'utilisation en bain-marie a parfois été évoquée, bien que des vases creux d'ouverture correspondante soient rares.

Les bols, suivant leur contenance bols, soupières ou saladiers dans lesquels des analyses pourraient indiquer des traces d'huile, pouvaient avoir la même destination que ceux qui constituent encore aujourd'hui le service de vaisselle courant ; mais, sur certaines formes, la présence de marlis empêchant d'y boire directement, il faut envisager l'usage de couverts, cuillères ou fourchettes - simples tiges pointues ? - suivant le contenu. Plus énigmatique est la présence des bols de petite dimension et plus encore des coupelles : il existait peut-être des récipients pour quelques friandises à côté des nourritures simples. La rareté des vases à verser est aussi remarquable : seulement deux cols à bec pincé suggèrent des cruches et trois becs tubulaires des formes plus pansues, DS.P. et commune grise confondues. Des brocs se trouvent plus fréquemment en avançant dans le Moyen Age.

\section{$\square$ Assiettes creuses}

La section de la panse dessine un quart de cercle dont la droite qui relie les points extrêmes fait avec l'horizontale un angle d'environ $50^{\circ}$.

- Formes 1 (fig. 10) : le $\mathrm{n}^{\circ} 14$ est peut-être une forme intermédiaire entre l'assiette et la coupelle. Les marlis d'un diamètre intérieur de moins de $15 \mathrm{~cm}$ y ont été joints : ils appartiennent probablement à des assiettes, aucun bol ou coupelle de cette dimension ne se trouve ici ; ailleurs, ils sont statistiquement rares (fig. 33). Seulement deux parties de pose sont conservées : un pied annulaire et un fond plat peu dégagé de la panse. D'autres variantes de fonds d'assiette sont représentées sur les figures $11,12,13$ et 14 .

Six marlis sont guillochés, trois imprimés, le $n^{\circ} 2$ au moyen du poinçon 4442 , un seul est profondément gravé d'une onde quadruple. Trois panses, les $n^{\circ} 7,13$ et 15 , présentent des guillochis sur la paroi extérieure. Ce décor se trouve rarement à cet emplacement ; deux seuls exemplaires sont répertoriés : un identique à Fréjus $\left(n^{\circ} 15152\right)$ et l'autre à
Saint-Blaise $\left(\mathrm{n}^{\circ} 5758\right)$ sur lequel les excisions sont plus profondes et très aiguës. Sur les $\mathrm{n}^{\circ} 7$ et 15 , comme sous le marli $\mathrm{n}^{\circ} 1$, ces traces sont certainement accidentelles.

- Formes 4 (fig. 11) : trois exemplaires classiques $\left(\mathrm{n}^{\circ} 16,17\right.$ et $18)$ et quatre variantes $\left(n^{\circ} 29,30,31\right.$ et 32$)$.

- Formes 8 (fig. 11) : un seul bord classique, le $n^{\circ} 20$, contrairement à d'autres sites ruraux du Var et du Vaucluse où ce profil est abondant. La lèvre du $n^{\circ} 19$ est atrophiée mais guillochée et soulignée d'une rainure.

\section{$\square$ Fonds d'assiettes décorés}

Deux fonds d'assiettes sont décorés de larges couronnes de guillochis (fig. 11, $\mathrm{n}^{\circ} 23$ et 26), ornement rare dans la production provençale. Cinq sont imprimés : le $n^{\circ} 24$ de la rouelle 4548 , le $n^{\circ} 25$ de la palmette provençale 3421 , le $n^{\circ} 22$ du petit arceau sommaire 4561 , le $n^{\circ} 21$ de la colonnette 4560 . Le fond entier $n^{\circ} 27$ présente le décor composé de la double rouelle 2015 , de l'arceau 4545 et de la croix 4544 ; son profil fruste (liaison continue entre la panse et le fond creusé d'une unique rainure) est inédit et n'est pas classique.

\section{$\square$ Assiettes plates}

Le rapport diamètre/profondeur est plus grand que pour les assiettes creuses, surtout à cause des diamètres importants de certaines de ces formes. Leur panse, de section presque rectiligne, est plus inclinée $\left(20\right.$ à $\left.35^{\circ}\right)$.

Les profils variés des lèvres (fig. 12) permettent de les rapprocher des bords en amande de la forme 8 ( $n^{\circ} 45$ et 47 ), parfois plus ou moins abâtardis ( ${ }^{\circ} 35$ et 37 ). C'est surtout à Apt que se retrouvent des parallèles à ces formes, principalement les profils à gorge interne $\left(n^{\circ} 33,39,41\right.$ et 43$)$ de forme 56 (Kauffmann 1987, 69, fig. 6).

Les parties de pose sont variées et vont du fond plat simplement rainuré $\left(n^{\circ} 46\right)$ au pied annulaire $\left(n^{\circ} 38\right)$ en passant par les gorges plus classiques ( ${ }^{\circ} 40$ et 42 ).

On retrouve sur le $n^{\circ} 37$ des guillochis accidentels. L'intérieur du $n^{\circ} 45$ est décoré de deux groupes de trois rainures circulaires concentriques.

$\square$ Fonds d'assiettes sommairement décorés (fig. 13)

Le souci de décoration se manifeste quelquefois par des éléments rudimentaires : le $\mathrm{n}^{\circ} 54$ est simplement strié de cercles concentriques, de même que les $\mathrm{n}^{\circ} 45$ et 48 (fig. 12), mais cette fois à l'extérieur comme à l'intérieur. Les $\mathrm{n}^{\circ} 55$ et 56 sont guillochés, ainsi que les $n^{\circ} 23$ et 26 (fig. 11).

$\square$ Fonds d'assiettes décorés au poinçon (fig. 14) - $n^{\circ} 57$ : une impression centrale du grand motif 4546 est entourée de quatre empreintes du “ $S$ " 4547 . Le fond est plat. 

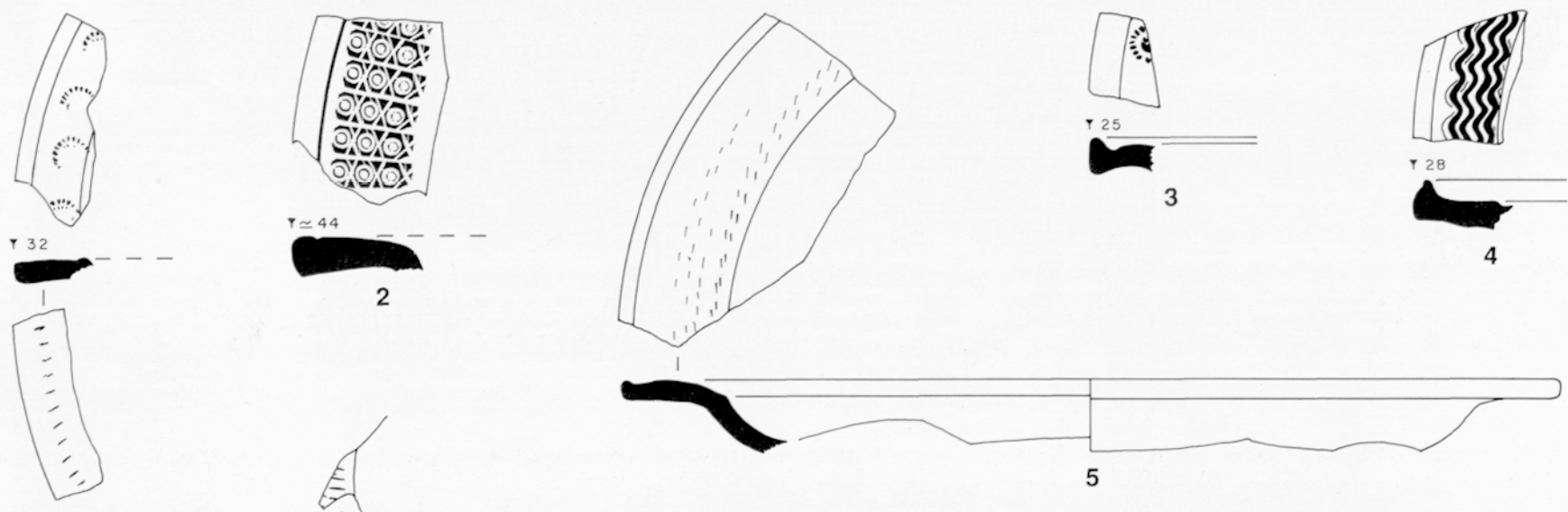

5
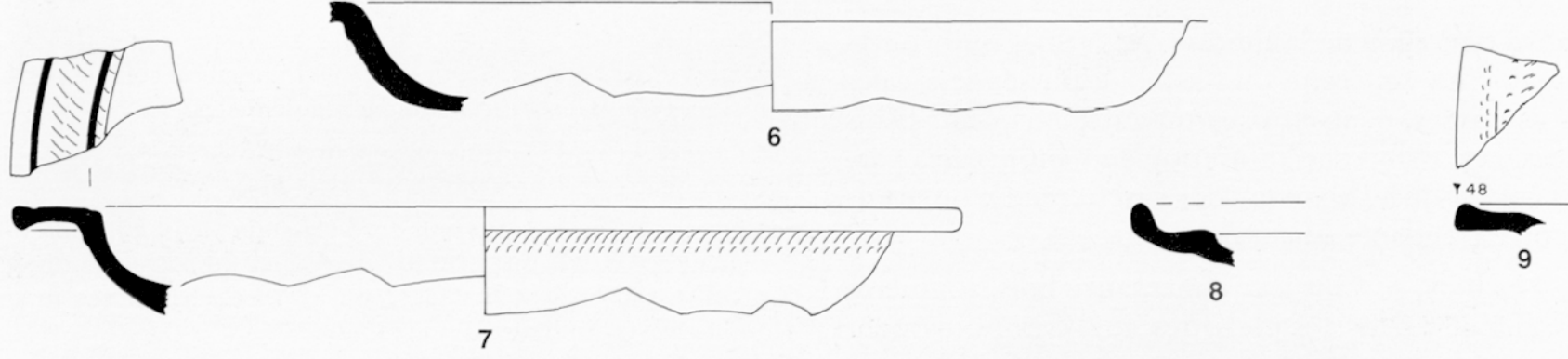

$\uparrow 48$

7
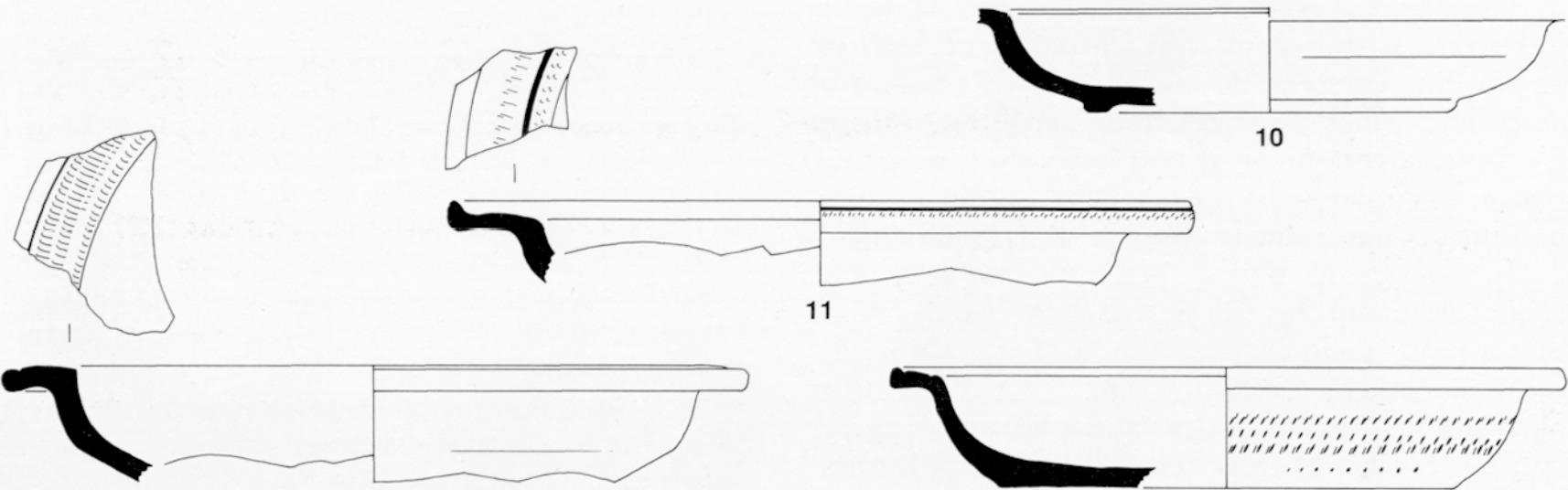

12

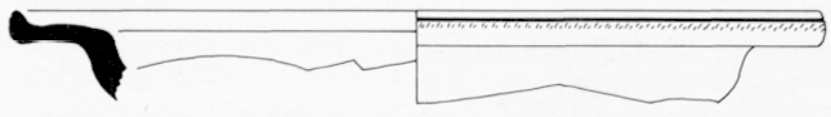

11
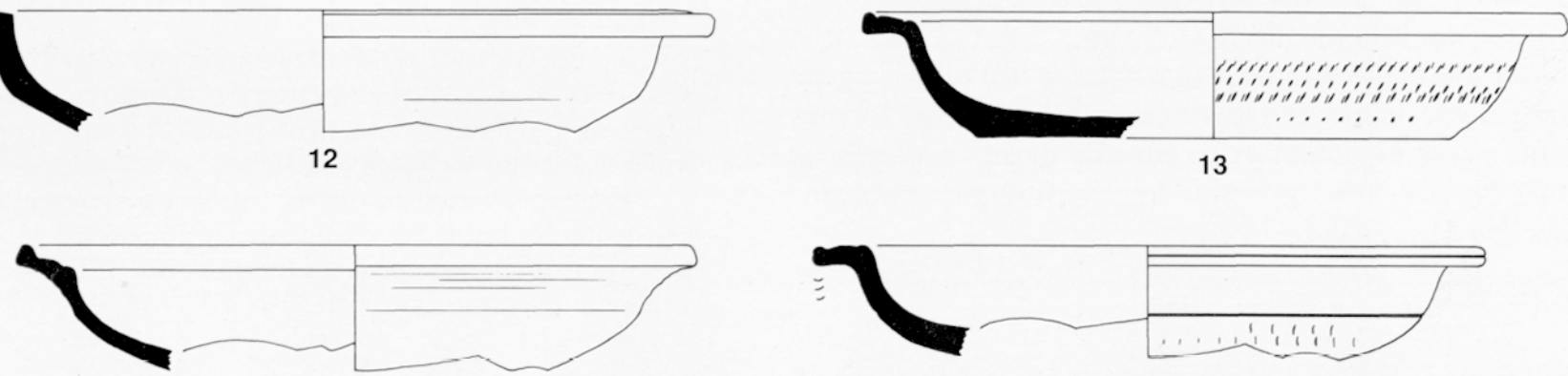

14

10 DS.P. Assiettes creuses. Formes 1 [éch. 1/3].

- $n^{\circ} 58$ : orangé ; un cercle entoure des impressions de la palmette 4552. Le fond plat est creusé d'une rainure.

- $\mathrm{n}^{\circ} 59$ : un cercle et la colonnette 4554 . Le pied est dégagé et creusé d'une large gorge.

- n 60 : rainures internes et partie de la grande palmette 4551. Pied classique creusé de deux gorges. Peut-être marseillais.

- $n^{\circ} 61$ : pièce rustique, de façonnage comme de décor ; rouelle 4559 .

Au total, on dénombre neuf fonds décorés au poinçon (voir fig. 11). $\square$ Récipients (coupes ?) de profondeur intermédiaire entre les formes hautes et plates (fig. 15)

Aucun exemplaire complet n'est connu. La différence avec les autres types est déterminée par l'inclinaison de la panse, mais la hauteur totale et par conséquent le diamètre du pied ne peuvent être évalués. De plus, aucune des bases retrouvées ne peut correspondre avec ces bords qui se terminent par une lèvre en amande ou arrondie. Un numéro ne sera attribué à cette forme que lorsque des profils reliant bord et pied pourront être établis. 


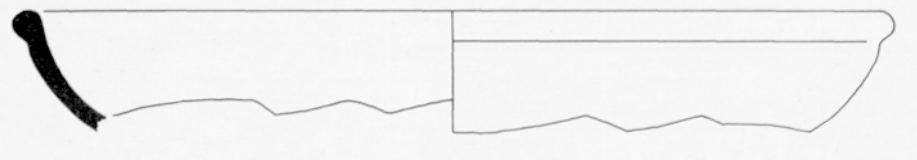

16

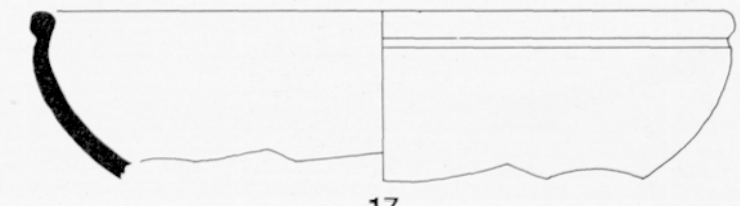

17
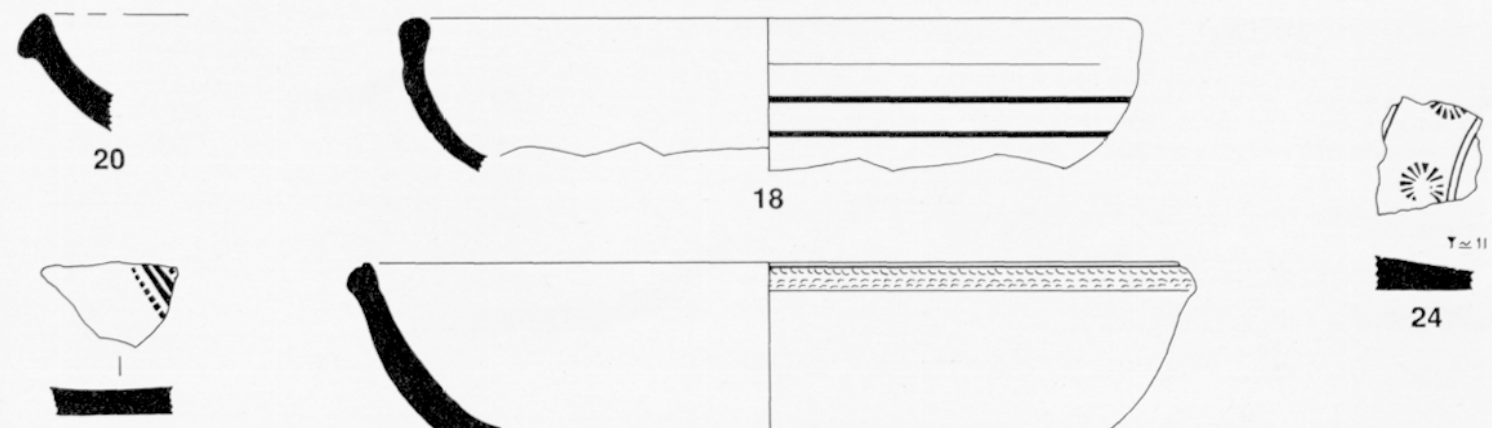

21
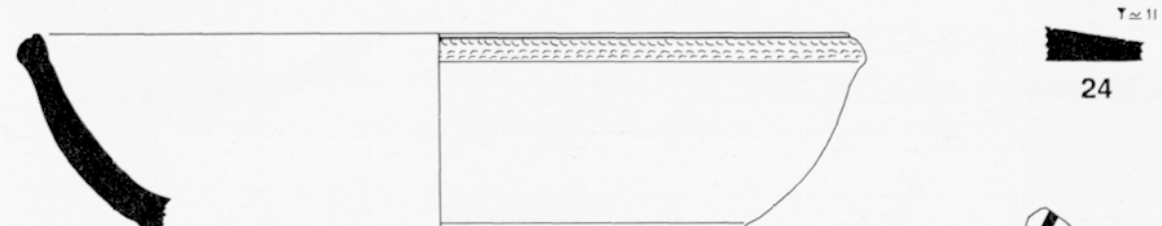

24

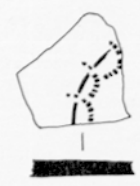

22

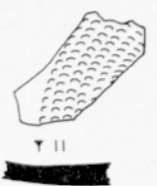

23

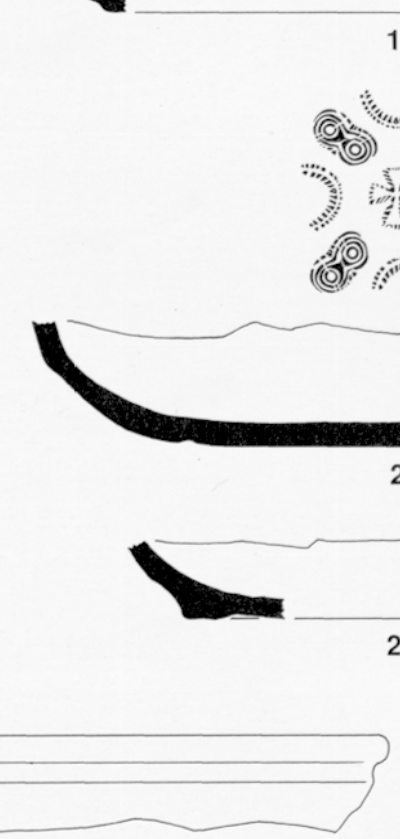

29

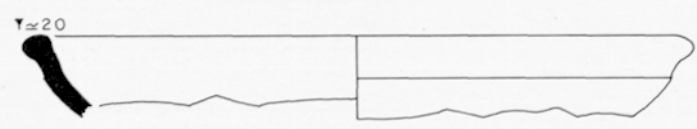

31
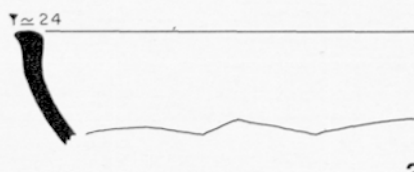

30

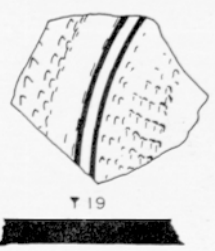

26

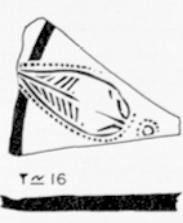

25

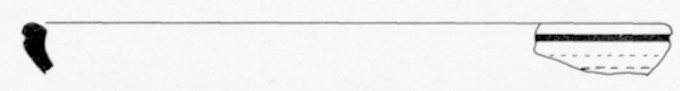

32

11 DS.P. Autres assiettes creuses. No 16,17 et $18:$ formes $4 ; 29,30,31$ et $32:$ variantes de la même forme ; 19 et $20:$ formes 8 [éch. 1/3].

\section{$\square$ Coupelles (fig. 16)}

Ce type de formes est particulièrement bien représenté ici par des profils variés dont deux formes $2\left(n^{\circ} 74\right.$ et 75 ), deux formes $52\left(n^{\circ} 79\right.$ et 85$)$, une forme $55\left(n^{\circ} 84\right)$, deux formes 70 ( $n^{\circ} 82$ et 83 ), une troisième (fig. $62, n^{\circ} 6$ ) est un exemplaire en pâte grossière.

Le profil du marli est semblable sur les deux formes 2 ; sur l'une il est guilloché ainsi que la panse du second. La lèvre et l'extérieur de la panse le sont aussi sur la forme 52 alors que le bas de la panse $n^{\circ} 85$ et de la forme 55 sont striés de rainures. Les traces de guillochis décelables à l'intérieur du $n^{\circ} 81$ sont accidentelles.

Le fond $n^{\circ} 86$ est imprimé autour d'un cercle central de quatre palmettes 4550 .

\section{$\square$ Bols}

- Forme 3 (fig. 17) : le diamètre intérieur est de 9 à $24 \mathrm{~cm}$. Quatre exemplaires sont décorés au poinçon : le $\mathrm{n}^{\circ} 94$ d'un motif illisible dont on peut se demander s'il s'agit réellement d'un outil gravé ou simplement de l'extrémité d'un objet 

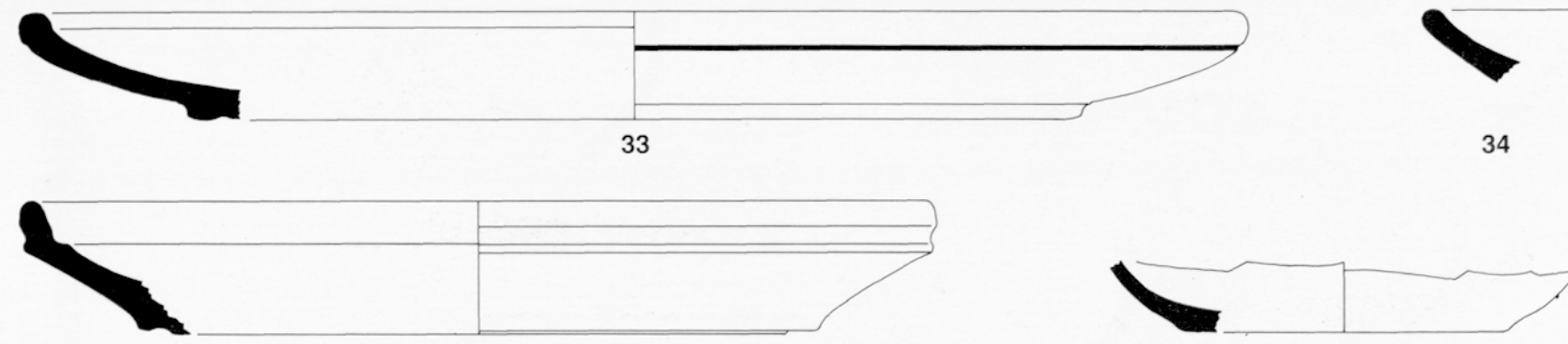

35
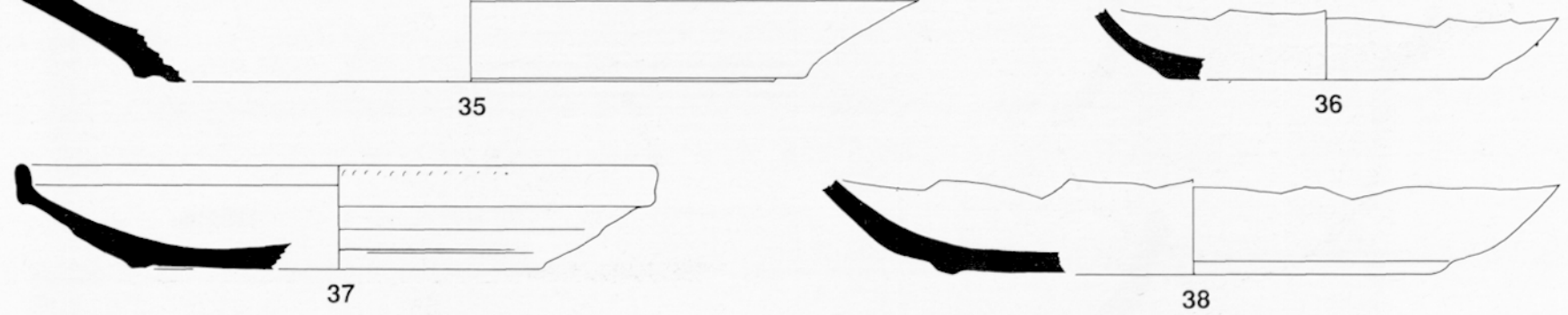

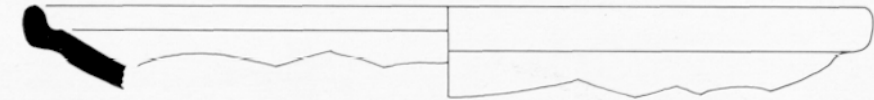

39

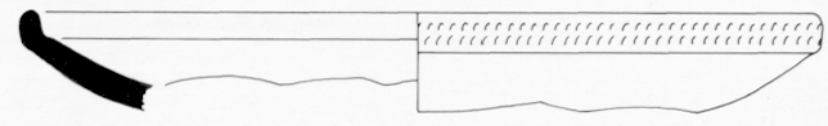

41

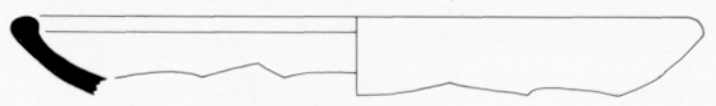

43

III III
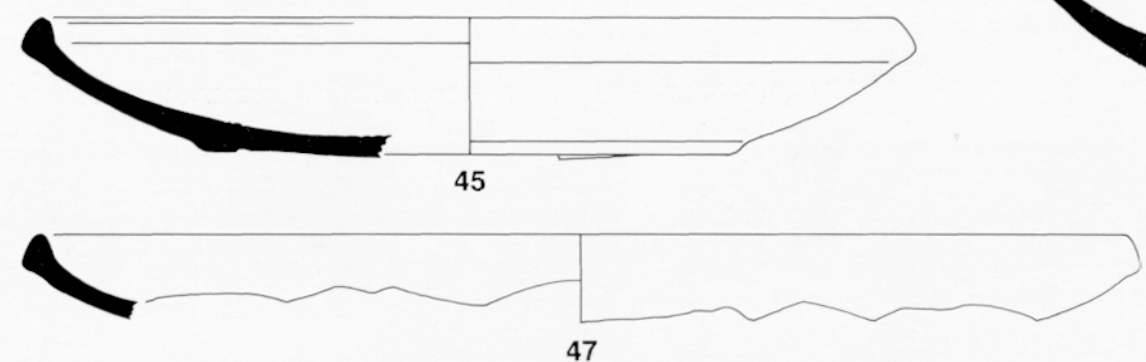

47
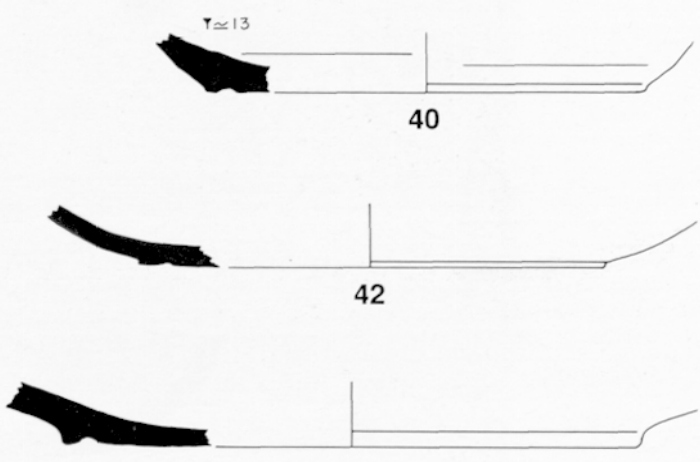

44
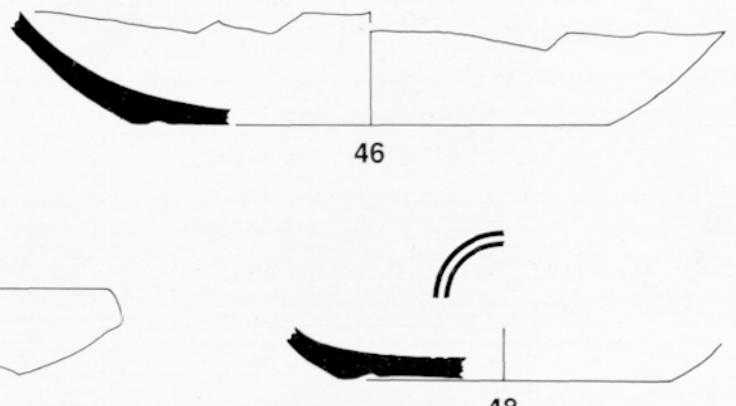

48
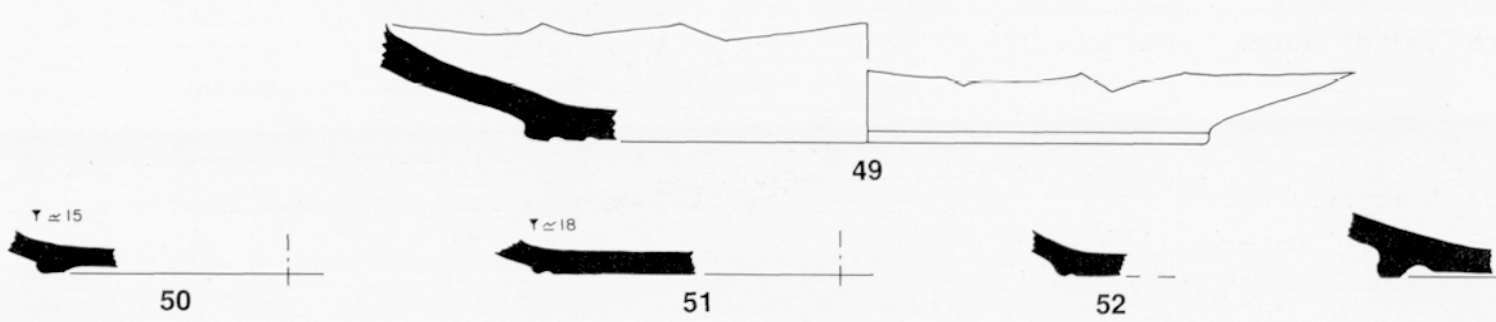

53

I 12 DS.P. Assiettes plates [éch. 1/3].

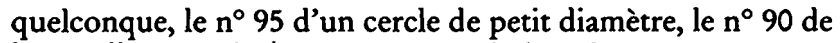
la rouelle 4236 , le fragment $n^{\circ} 92$ de la colonnette 4442 que l'on retrouve plus complète sur le marli de forme $1 n^{\circ} 2$. Le $\mathrm{n}^{\circ} 93$ est orangé, fin et large ; il porte des empreintes sur deux rangs d'une rouelle peu lisible, la surface étant fortement écaillée.

Cinq marlis sont guillochés ainsi que trois panses. Les faibles traces sur le $n^{\circ} 91$ sont accidentelles. Sur le $n^{\circ} 98$, les 


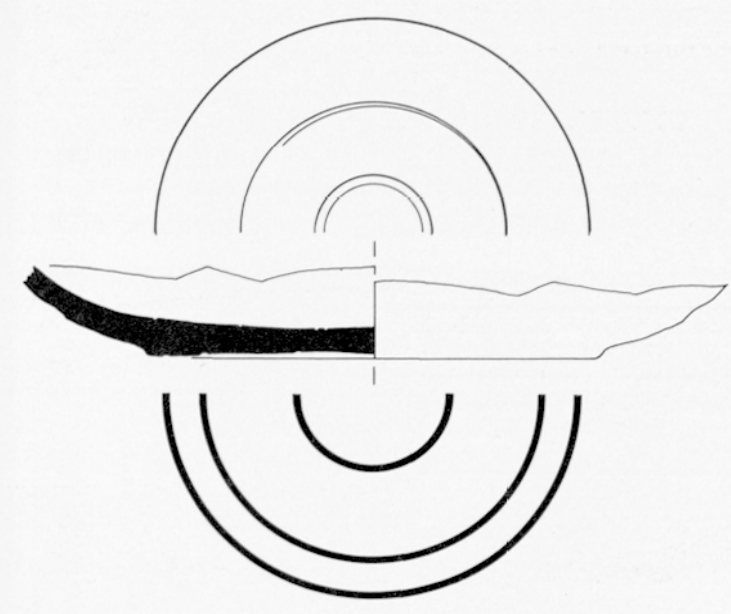

54

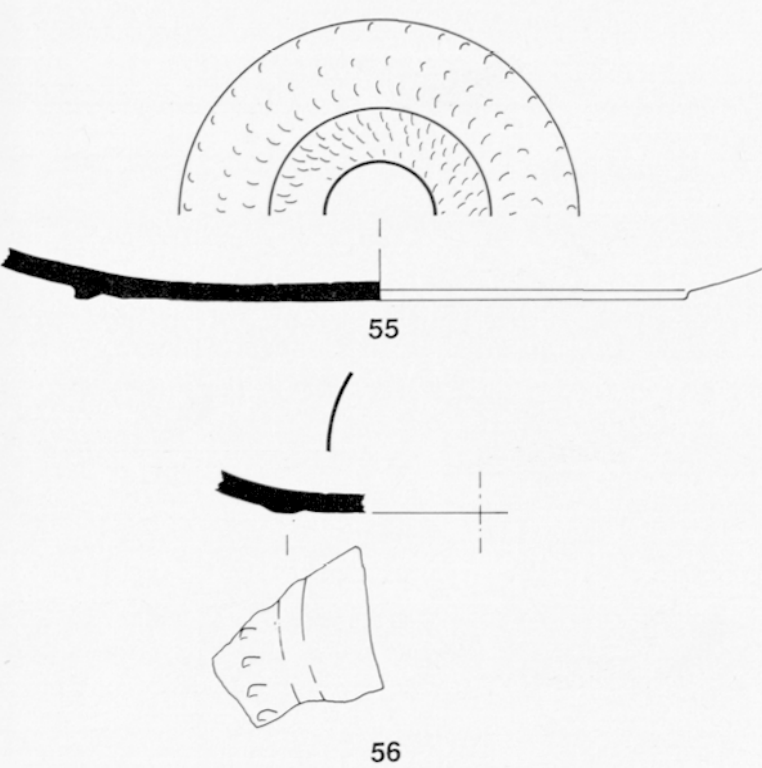

11 DS.P. Fonds d'assiette sommairement décorés [éch. 1/3].

14 DS.P. Autres fonds décorés au poinçon [éch. 1/3].

excisions sont en forme de "coups d'ongle" et se retrouvent sur et sous le marli et sur la panse.

Il est vraisemblable que les trois fonds isolés appartiennent à cette forme en raison de leur galbe ; le $n^{\circ} 105$ conserve d'ailleurs une amorce de marli.

- Forme 3c (fig. 18) : un fragment représente cette forme qui, bien que rare, se rencontre dans presque toutes les fouilles de quelque importance avec deux autres pièces trouvées hors de la fosse (fig. $31, \mathrm{n}^{\circ} 4$ et 5 ; fig. 32 ).

- Forme 6 (fig. 19) : ces profils variés ont une lèvre plus ou moins marquée : absente sur le $n^{\circ} 110$, très saillante sur le $n^{\circ} 114$ ou qui rappelle le style languedocien sur les $n^{\circ} 111$, 118 et 126.

Trois pièces sont décorées au poinçon : le $\mathrm{n}^{\circ} 108$ porte, disposée en chevrons, la grande colonnette 4549, le $n^{\circ} 112$ la
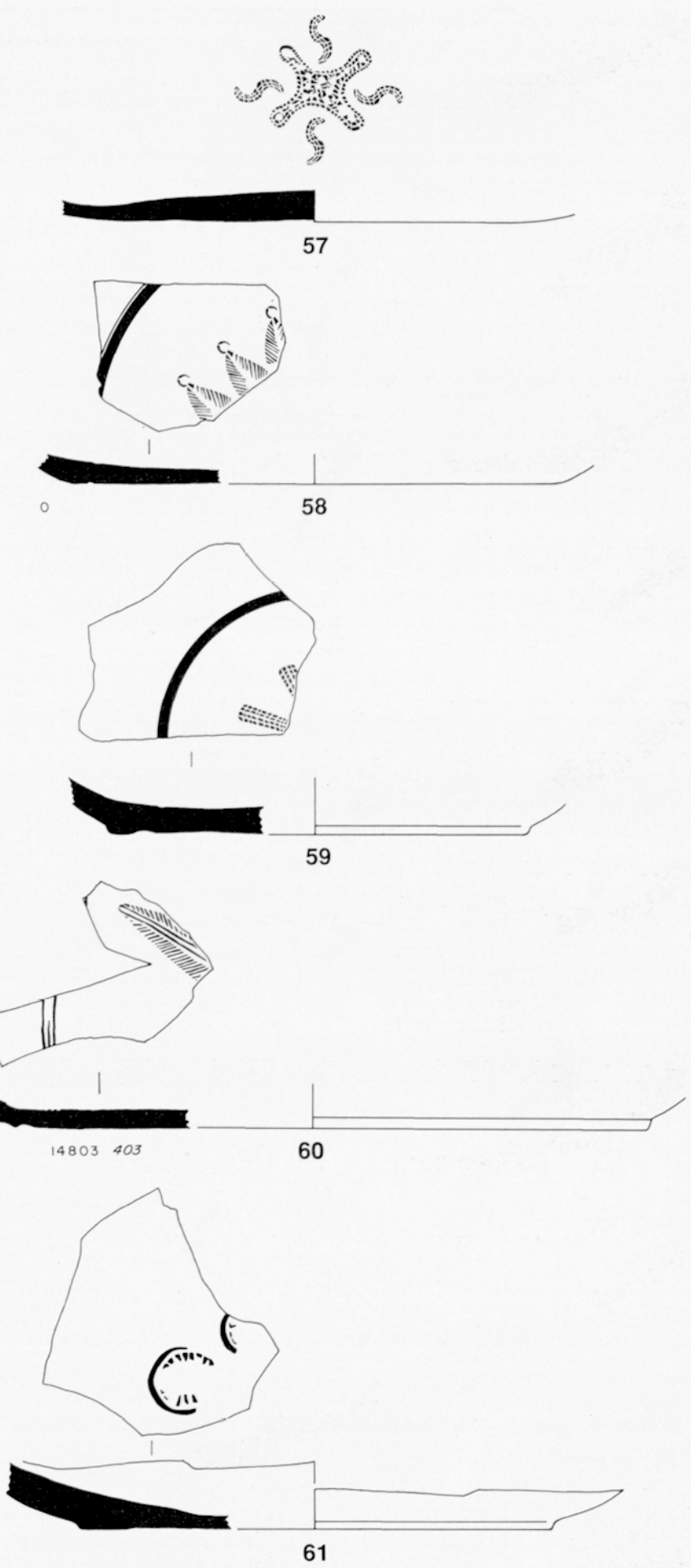

rouelle 4555 et le $n^{\circ} 110$ la palmette 2340 . Ce dernier décor et la couleur orangée du tesson permettent d'attribuer sans équivoque cette pièce à une production homogène dont un nombre important de pièces ont été trouvées dans les fouilles de Saint-Julien-les-Martigues (B.-du-Rh.) (Rigoir 1985). Il s'agit donc d'une proche importation.

Trois autres sont guillochées. Les traces visibles sous la lèvre du $n^{\circ} 111$ sont accidentelles. Le $n^{\circ} 109$ porte une trace d'ondè. Un autre exemplaire, orangé, provient de l'extérieur de la fosse (fig. $31, n^{\circ} 2$ ). 


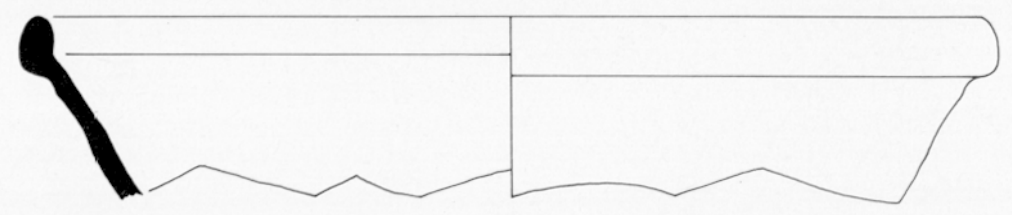

62

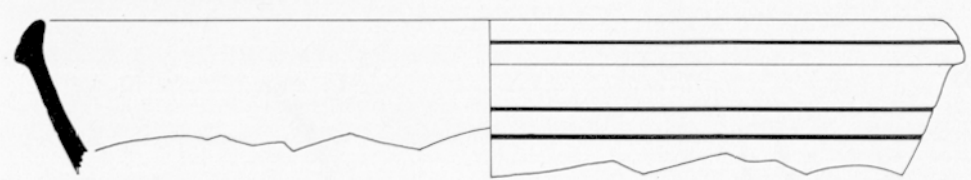

63

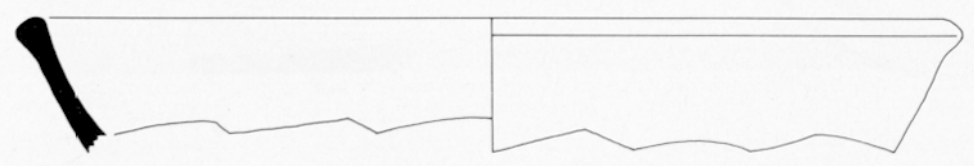

64

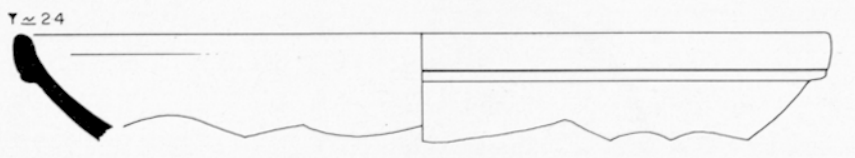

65

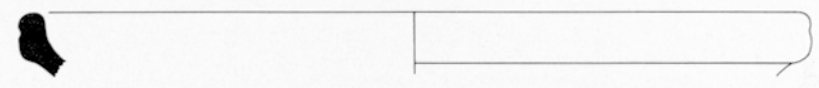

66

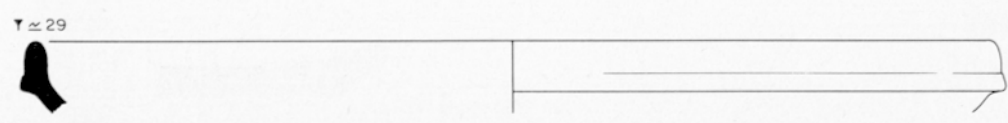

67

69
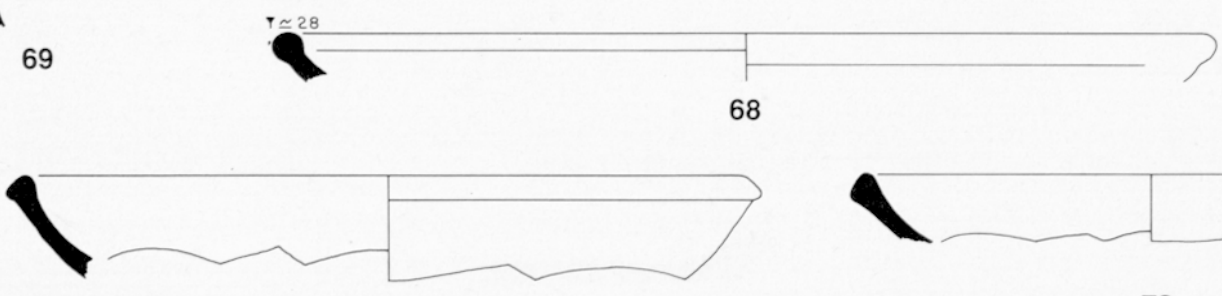

71

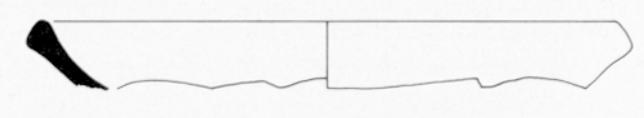

72

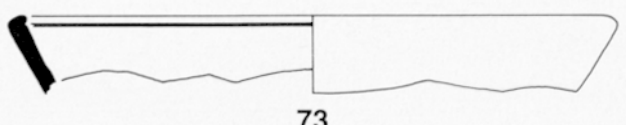

115 DS.P. Récipients (coupes ?) de profondeur intermédiaire entre les formes hautes et plates [éch. 1/3].

- Forme 18 (fig. 20) : deux fragments révèlent le profil type des productions classiques marseillaises (répertoriées OLV/OSL dans le catalogue des formes Rigoir 1985), les $\mathrm{n}^{\circ} 146$ et 149 . Bien que moins précisément dessinés, les $n^{\circ} 127,132$ et 134 appartiennent peut-être à cette production. Les autres profils sont peu caractéristiques.

Trois sont décorés au poinçon : le col $n^{\circ} 130$ de l'arceau 4553 qui se retrouve sur la forme à bec tubulaire $n^{\circ} 222$. Les deux autres fragments sont très corrodés, ce qui interdit toute identification d'une palmette sur le $\mathrm{n}^{\circ} 139$ et d'une colonnette sur le $n^{\circ} 140$.

Huit pièces sont guillochées, sur le bord et/ou sur la panse. Comme sur les autres formes, ces guillochis sont parfois peu nets.

$\square$ Panses décorées (fig. 21)

Le $n^{\circ} 165$ appartient à une grande panse cylindrique. Un seul fragment $\left(n^{\circ} 164\right)$ est décoré au poinçon : colon- nettc 4557. Les guillochis sont généralement flous, de dessin varié, donc exécutés avec des outils différents ou tenus différemment.

\section{$\square$ Cruches}

- Le $\mathrm{n}^{\circ} 174$ est un haut de forme $26 b$ (fig. 22). Le bec verseur est nettement pincé, le raccord col/panse bien marqué ; l'anse est tournée. Le profil de la lèvre du $n^{\circ} 171$ rappelle celui de la forme 63 (fig. 25) mais le col est plus long, vertical et la paroi est fine.

Trois autres fragments représentent vraisemblablement trois objets distincts, dont une grande forme $\left(n^{\circ} 175\right)$.

Un pot à deux anses, dont il ne manque qu'une de cellesci, a été trouvé à quelque distance de la fosse (fig. 31, $\mathrm{n}^{\circ} 3$ ).

- Forme 63 (fig. 25) : le profil complet est connu grâce à un exemplaire inédit de Saint-Jean-de-Garguier (B.-du-Rh.) dont la contenance est de 6,39 l (fig. 26). 


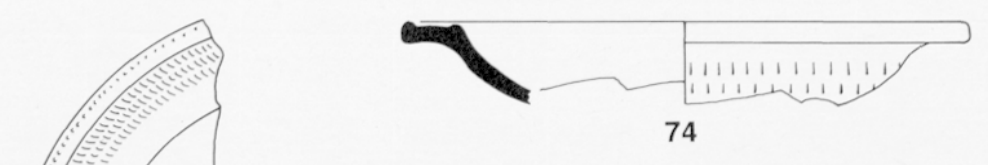

74

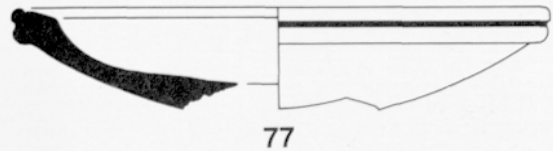

77

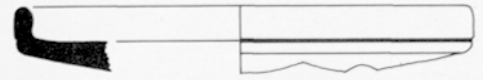

78

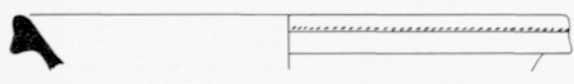

82

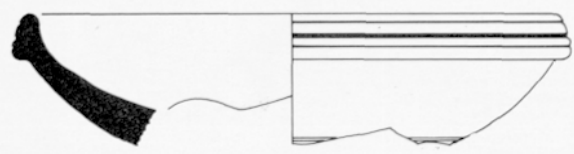

85
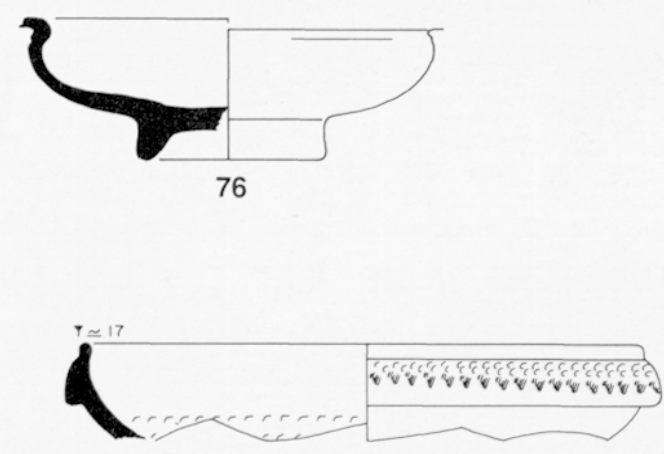

79

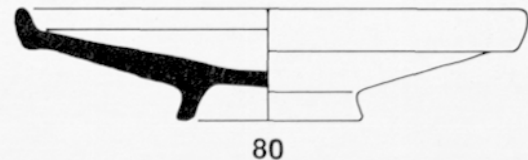

81

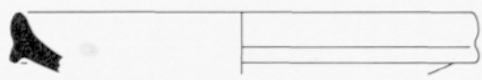

83

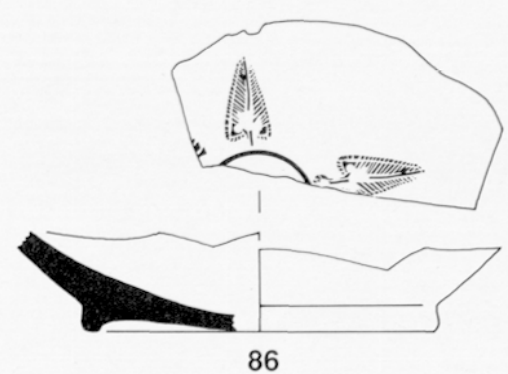

84

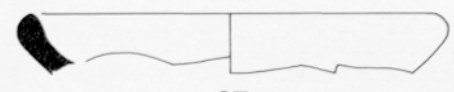

87

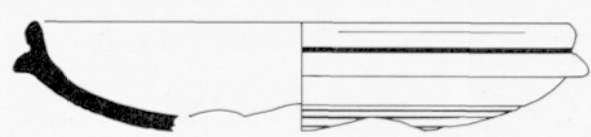

116 DS.P. Coupelles. No 74 et $75:$ forme $2 ; 87:$ forme $5 ; 79$ et $85:$ forme $52 ; 84:$ forme $55 ; 82$ et 83 : forme 70 [éch. 1/3].

Le col d'une pièce d'assez grande dimension (fig. 62 , $\mathrm{n}^{\circ} 1$ ), plus grand que celui de Saint-Jean, témoigne de l'emploi de pâtes grossières dans la fabrication des DS.P.

$\square$ Becs tubulaires (fig. 27)

Ces appendices se trouvent appliqués sur des formes de profils variés : formes $29,36 \ldots$ et leur position par rapport à la lèvre semble un indice chronologique (Bonifay 1986, 217). Il ne s'agirait pas, d'après ces observations, d'exemplaires très tardifs. Le $\mathrm{n}^{\circ} 221$ est particulièrement long. Les deux parois sont décorées, le $n^{\circ} 221$ du " $S^{\prime}$ " 4556 et le $n^{\circ} 222$ de l'arceau 4553 qui décore aussi le bol $\mathrm{n}^{\circ} 130$ (fig. 20).

$\square$ Pieds divers (fig. 23)

La majorité de ces bases présente un bourrelet annulaire bas sauf le $n^{\circ} 180$ qui est plus élevé (coupelle ?) et les $n^{\circ} 189$,
194, 197 et 199 qui sont plats et appartiennent peut-être à des formes 63. Parmi les premiers, trois supportent des panses guillochées.

\section{$\square$ Mortiers (fig. 24)}

Aucun profil classique parmi ces fragments de forme 29; le moins bâtard est le $n^{\circ} 203$. Les deux parties de versoir conservées, $\mathrm{n}^{\circ} 204$ et 210 , sont des becs pincés et non pas découpés comme le $n^{\circ} 4$, fig. 52 de céramique commune grise. Le listel atrophié du $n^{\circ} 211$ et son petit diamètre le rapprochent des formes tardives de Saint-Blaise (Démians d'Archimbaud et al. à paraître, fig. $95, \mathrm{n}^{\circ} 121$ et 124 ; fig. 96, $\mathrm{n}^{\circ}$ 129).

Les fonds présentent soit des inclusions de basalte $\left(n^{\circ} 212\right.$ à 214$)$, de quartz $\left(n^{\circ} 215\right.$ et 216$)$ ou de terre cuite broyée $\left(\mathrm{n}^{\circ} 217\right)$. 


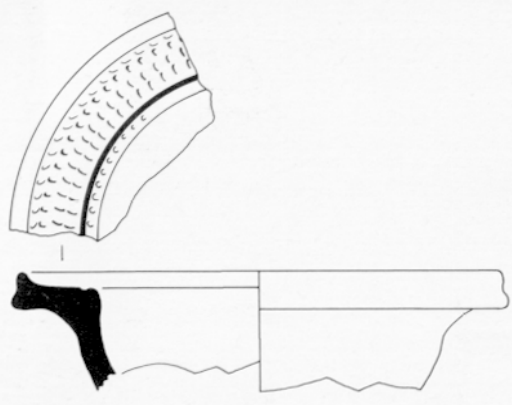

88
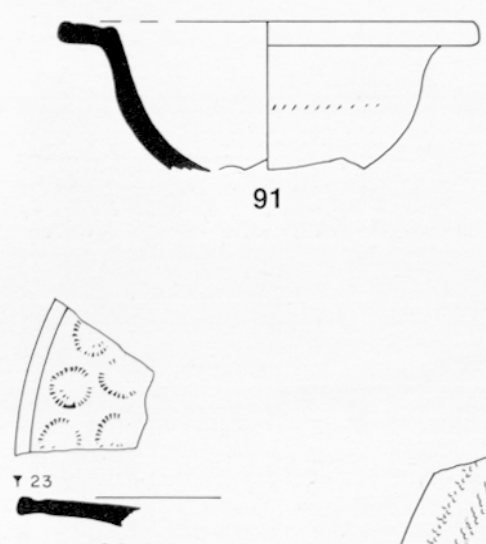

93
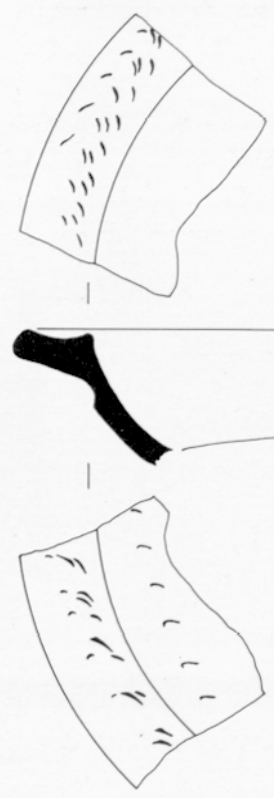

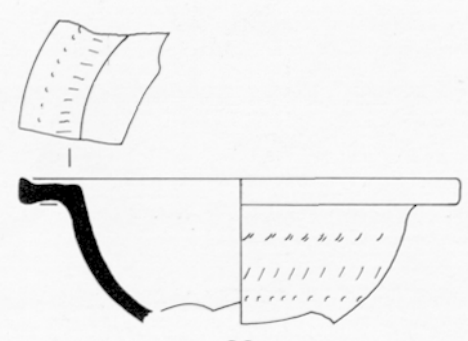

89

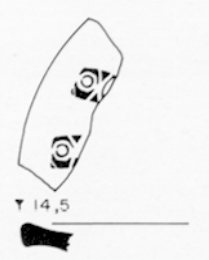

92
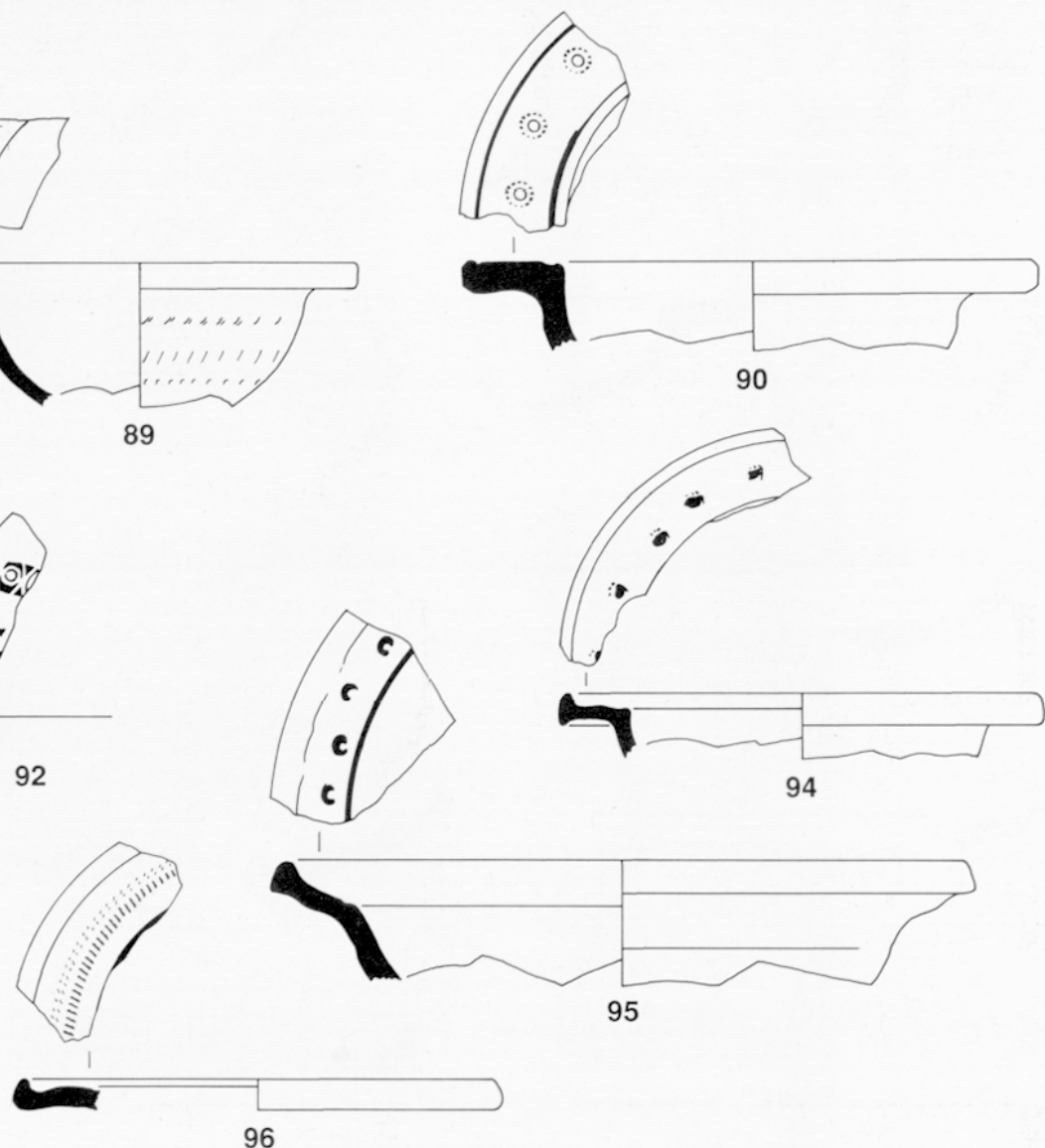

96

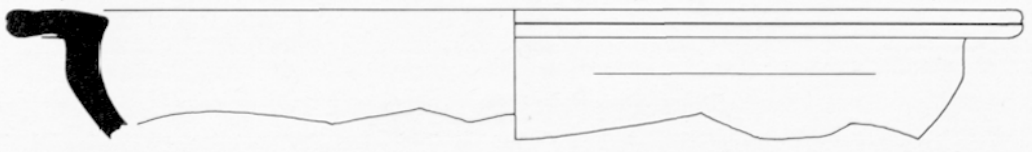

97

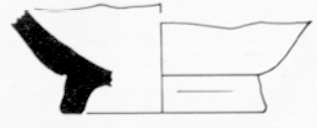

103

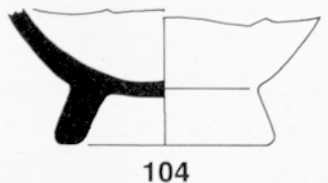

100

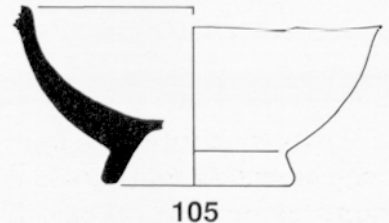

11 DS.P. Forme 3. Bols à marli de dimensions et de profils variés [éch. 1/3].

11 DS.P. Forme 3c. Grand bol à marli façonné de dépressions digitales verticales internes. Sur tous les exemplaires connus, on remarque au moins une rainure horizontale sous les dépressions. Deux autres pièces ont été trouvées hors de la fosse (fig. $31, n^{\circ} 4$ et 5 ; fig. 32) [éch. 1/3].

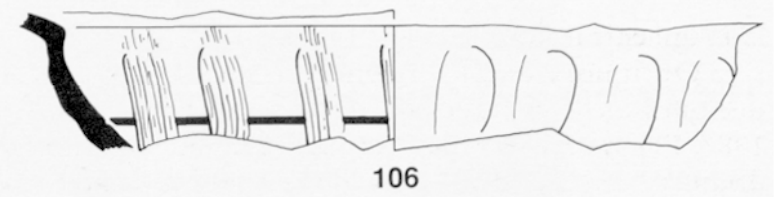




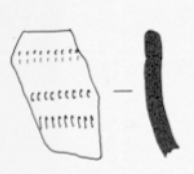

107

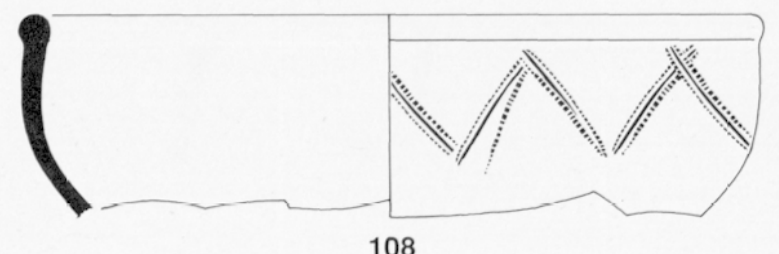

108
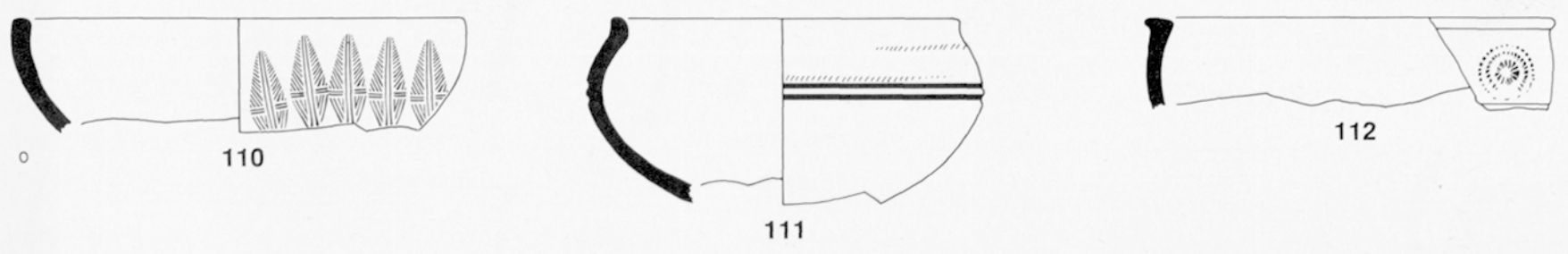

112
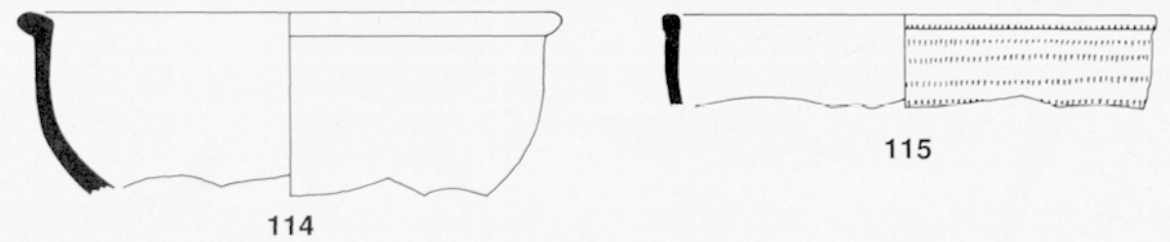

115

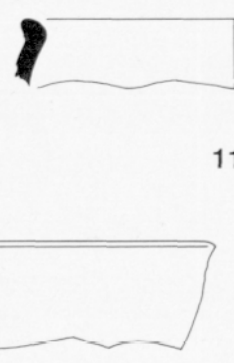

121

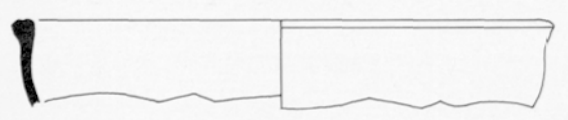

123

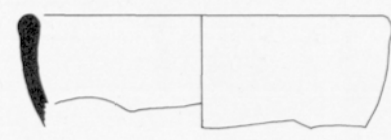

117
122

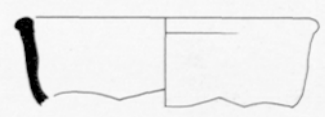

119
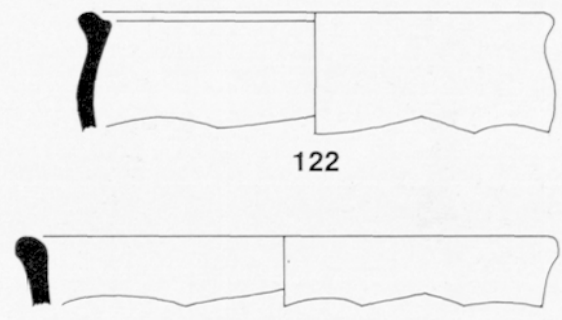

125

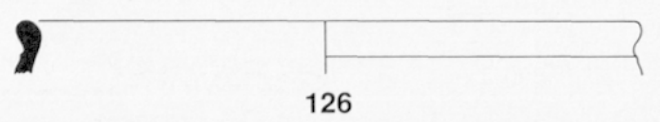

19 DS.P. Forme 6. Un autre exemplaire, orangé, provient de l'extérieur de la fosse (fig. $31, n^{\circ} 2$ ) [éch. 1/3].

Un fragment de fond, trop petit pour être dessiné, est soigneusement tournassé à l'extérieur et est souligné de deux rainures peu profondes. Il est similaire à celui trouvé à Notre-Dame-de-la-Seds (Aix-en-Provence).

$\square$ Couvercles (fig. 28)

Deux formes 54, à emboîtement, les $n^{\circ} 223$ et 224 . Le second est plus aplati. Sur le bouton du premier se voit nettement la trace du décollement à la ficelle. Les $n^{\circ} 225$ et 226 sont vraisemblablement des bords de couvercles à poser.

\section{$\square$ Fragments isolés}

On ignore quelles formes pouvaient supporter les quelques pieds coniques recensés (par exemple Kauffmann $1987,71)$, et comment se poursuivaient le fragment cylindrique $n^{\circ} 228$ (fig. 29) - peut-être un pied de lampe ou d'un calice inspiré de la forme Hayes 170.1 de sigillée claire D ou le fragment de tube de la figure 30.

\subsubsection{Origine}

Manipuler des poteries documente sur le mode de vie et, plus intimement, nous renseigne sur les ateliers qui les ont fabriquées. Les mains qui ont façonné pour une importante proportion les DS.P. de Gardanne se manifestent pour la première fois, exception faite de fabrications "marseillaises" et de quelques pièces dont les analyses en cours doivent déterminer si elles sont analogues à des exemplaires 


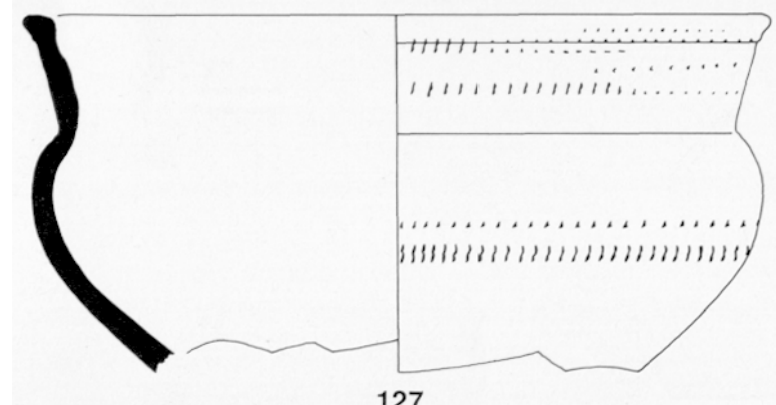

127
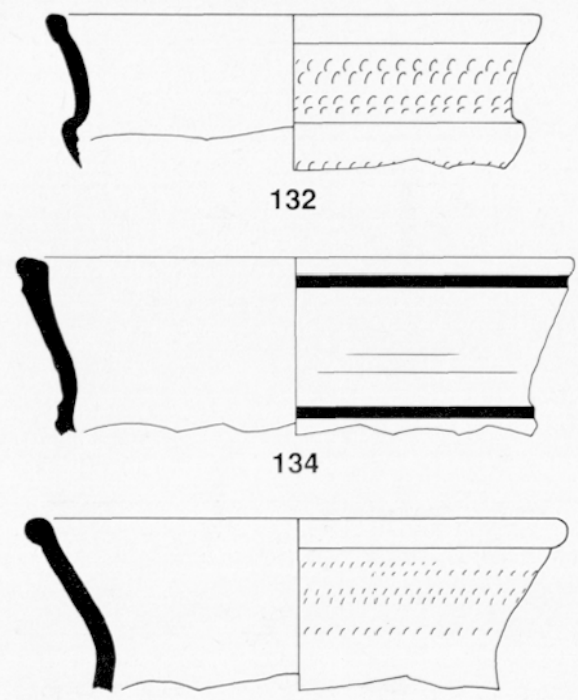

137

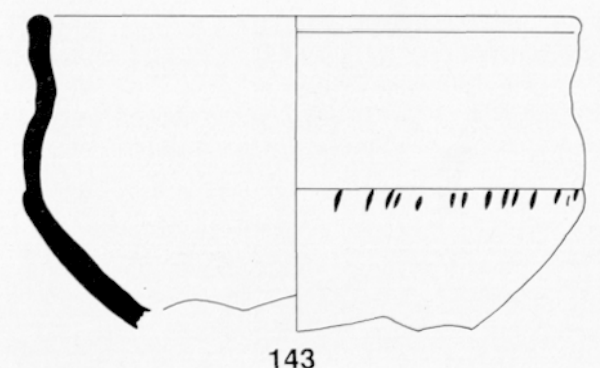

143
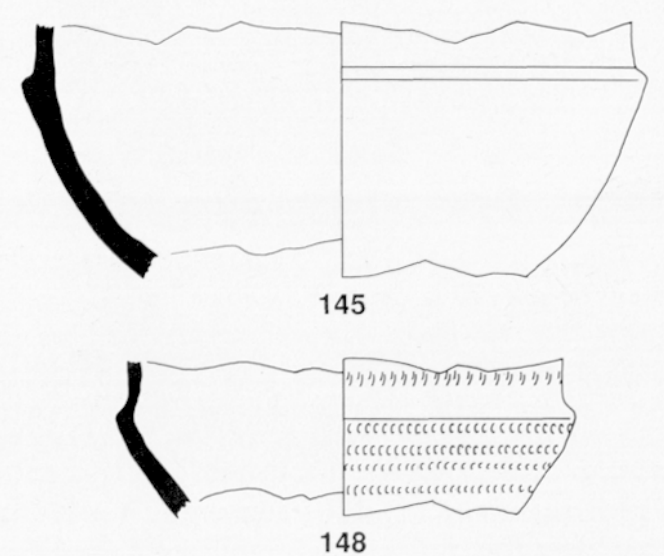
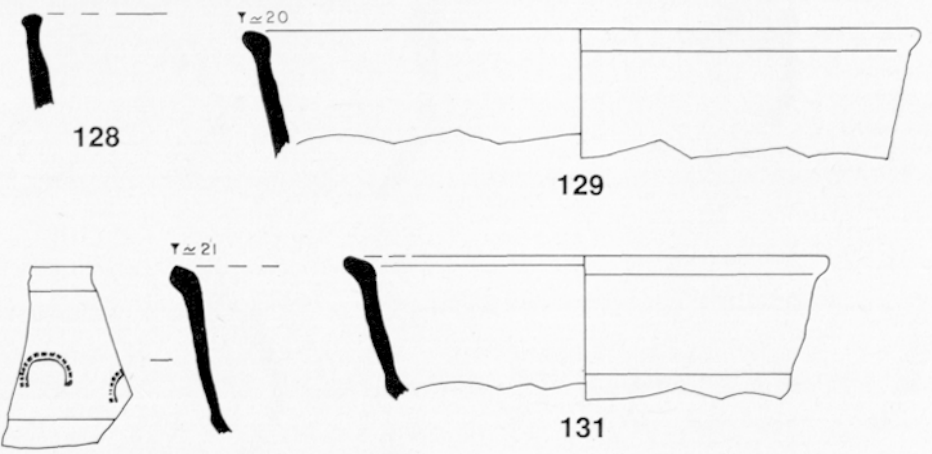

130

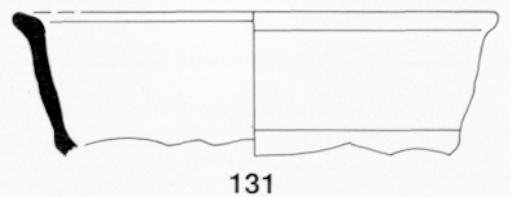

131

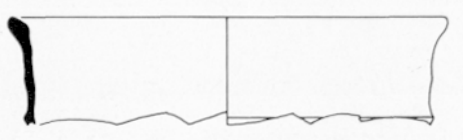

133

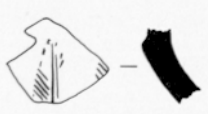

139

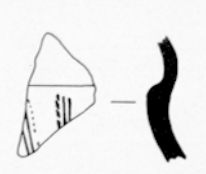

140

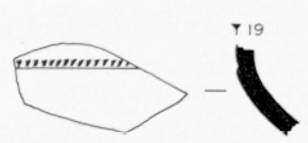

141

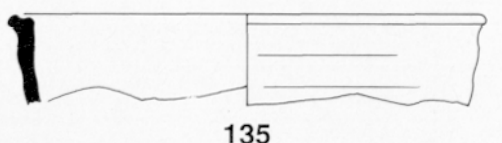

135

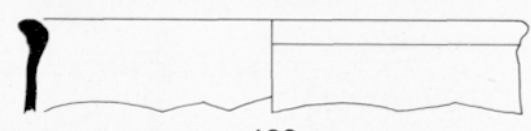

136

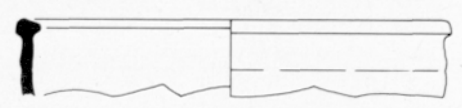

138

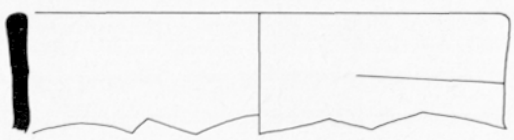

142
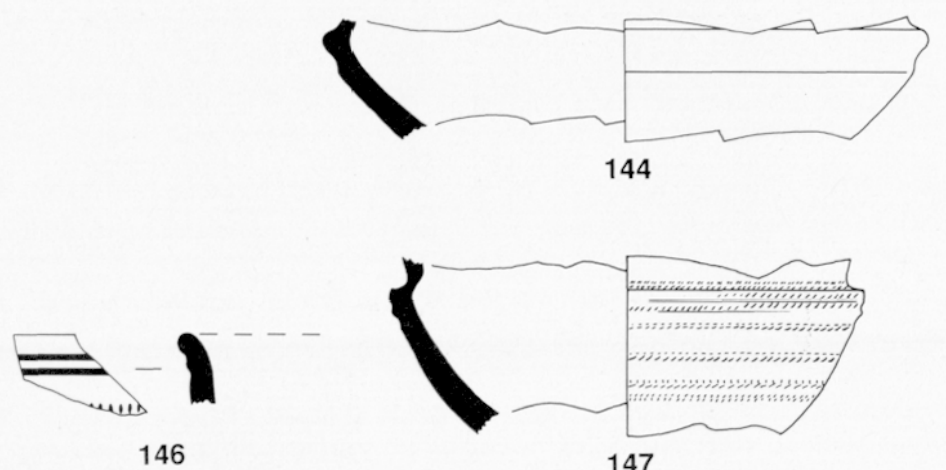

147
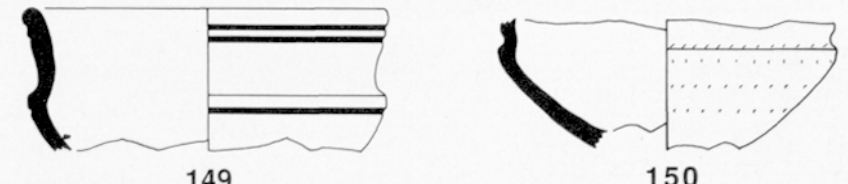

20 DS.P. Forme 18. Ensemble peu homogène. Les bords isolés semblent pouvoir être attribués à la même forme [éch. 1/3]. 

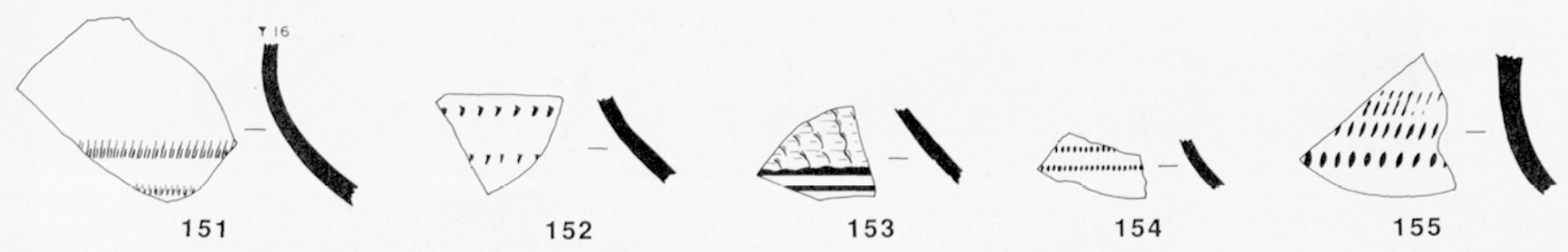

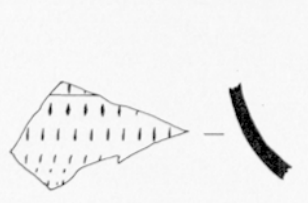

156

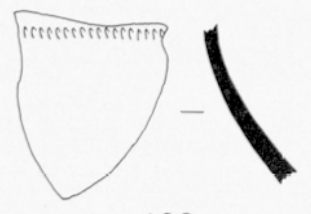

160

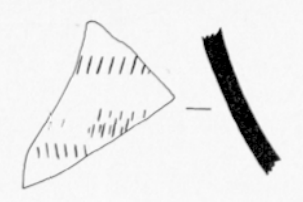

157

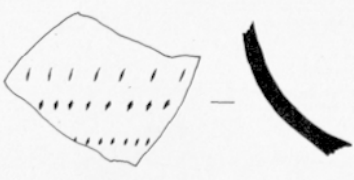

158
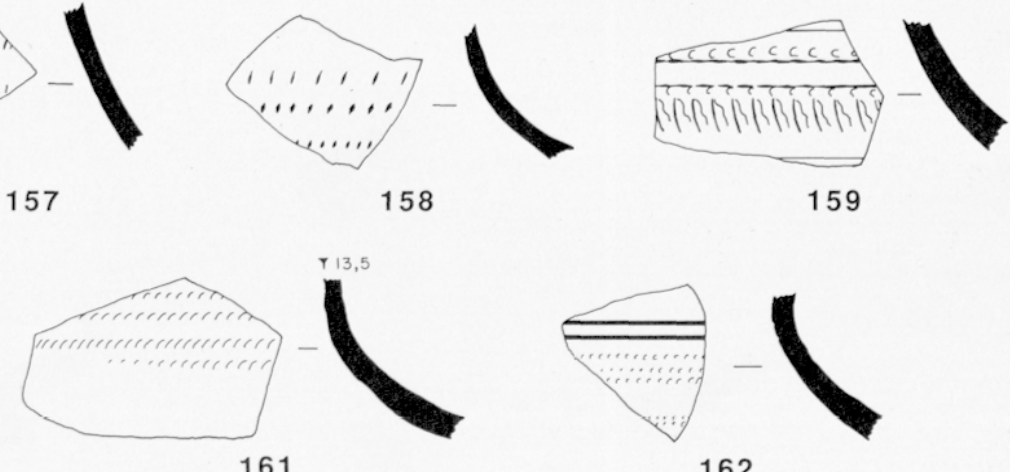

162

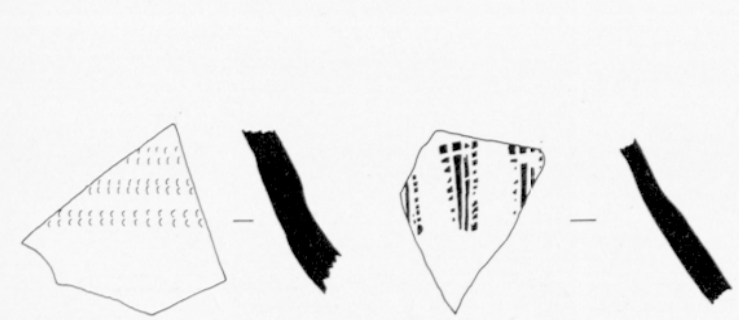

163
164

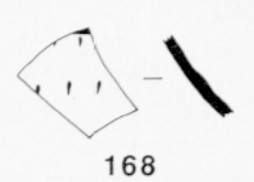

161

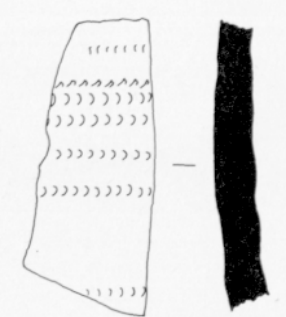

165

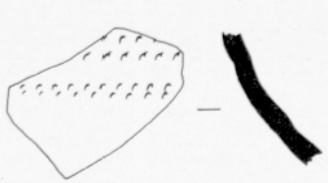

166
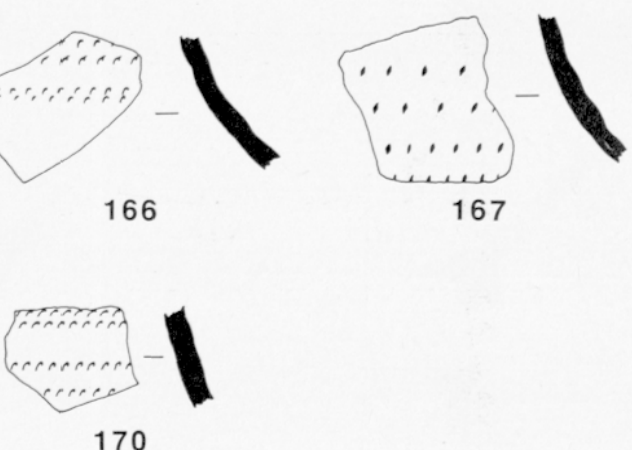

170

169

69

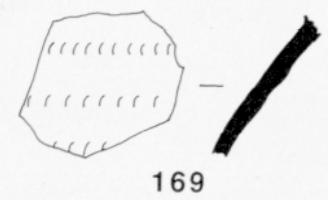

121 DS.P. Panses décorées. № 151 à 162 : fragments de formes ouvertes (intérieur lissé) ; 163 à 170 : fragments de formes fermées (traces de tournage subsistant à l'intérieur) [éch. 1/3].

122 DSP. Cruches. N ${ }^{\circ} 174$ : haut de forme $26 b$.
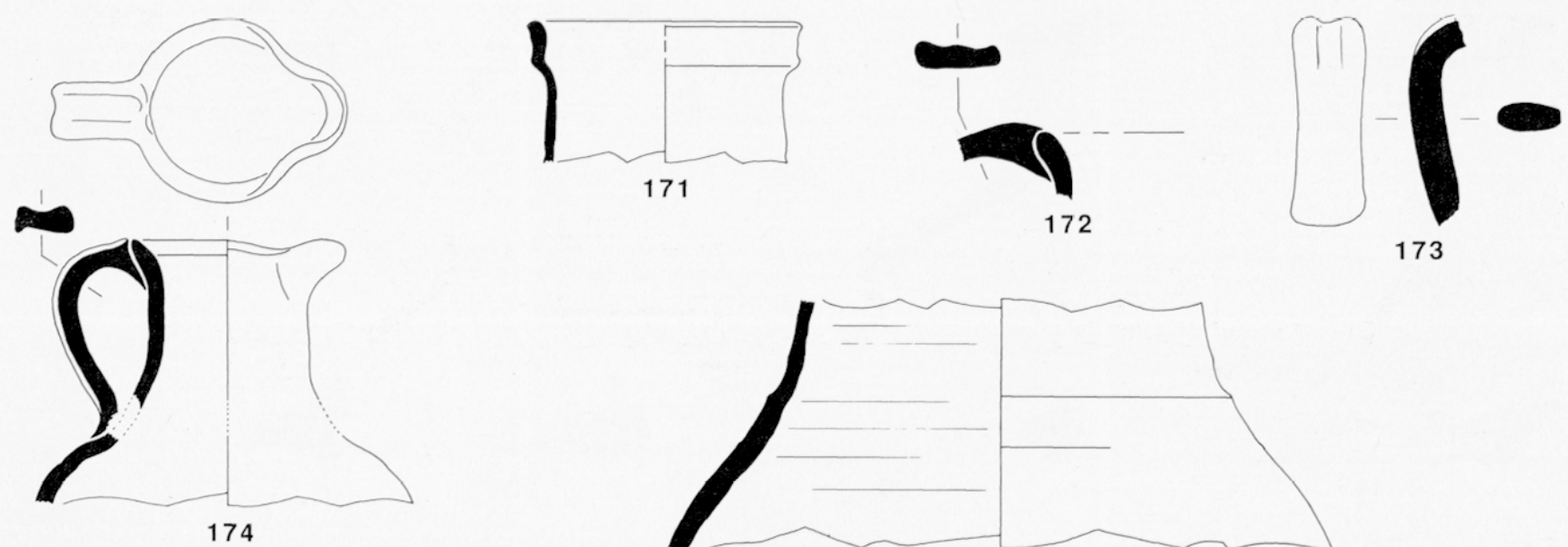

173

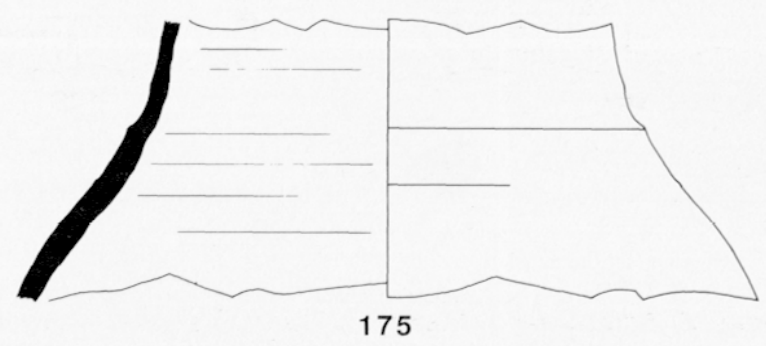



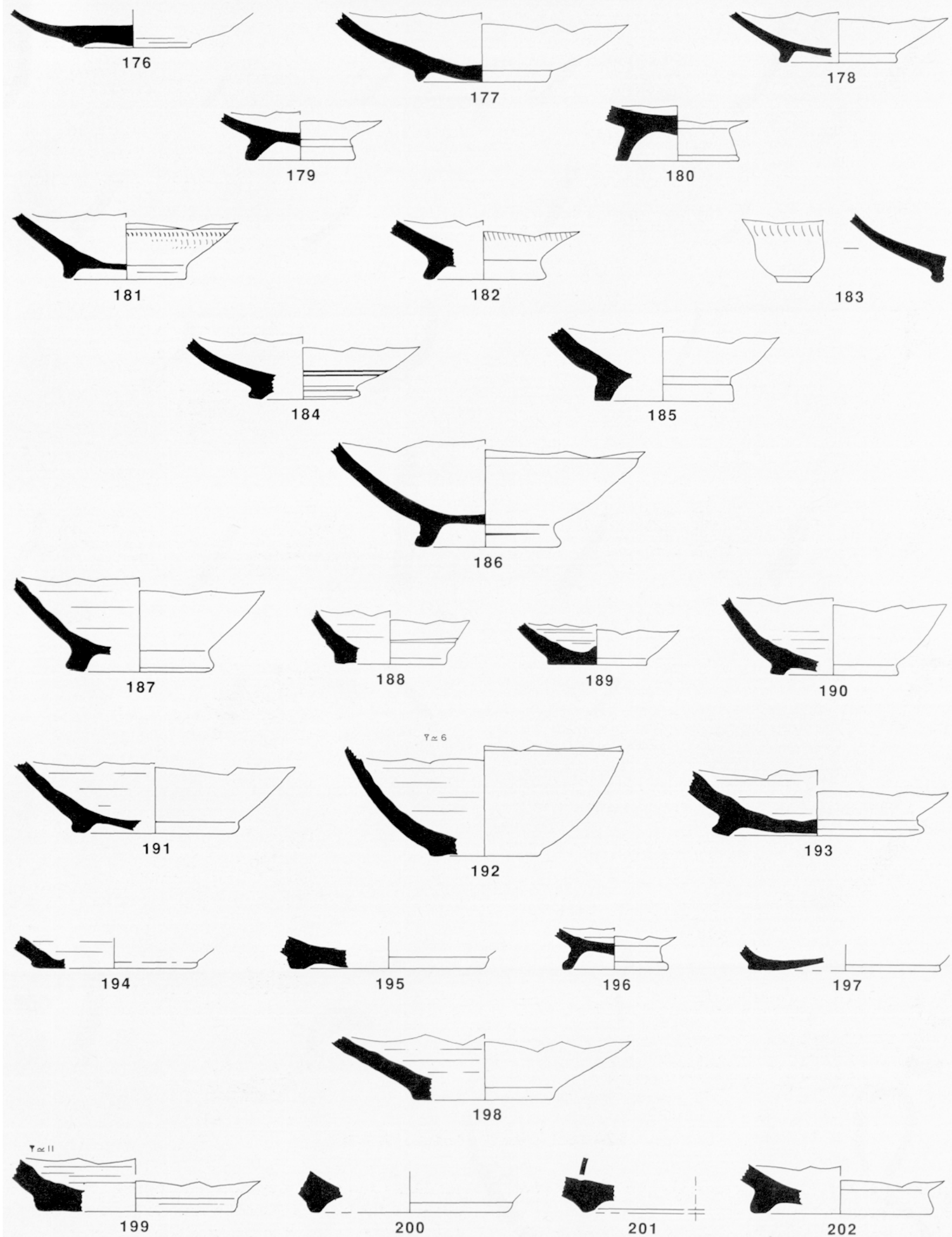

123 DS.P. Pieds divers. № 176 à 186 : vases ouverts ; 187 à 199 : vases fermés. Mêmes observations que pour les fragments ue panse [éch. 1/3]. 

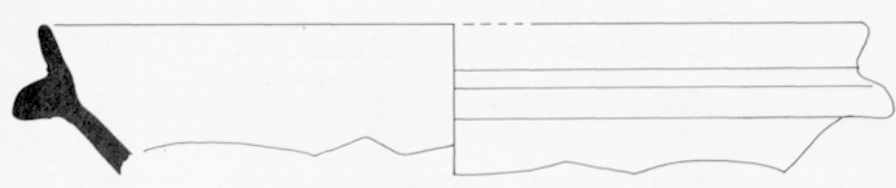

203
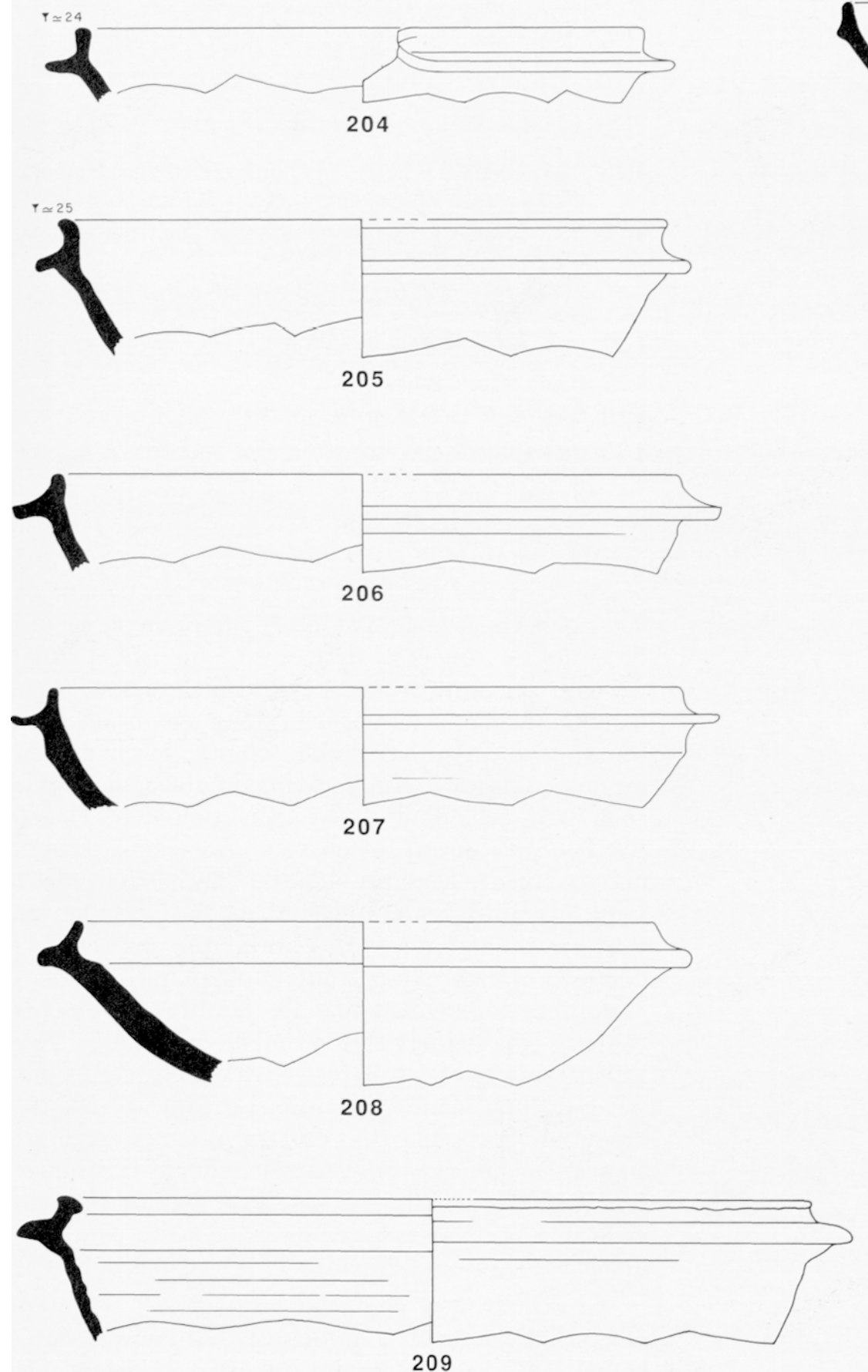

24 DS.P. Forme 29. Mortiers [éch. 1/3].
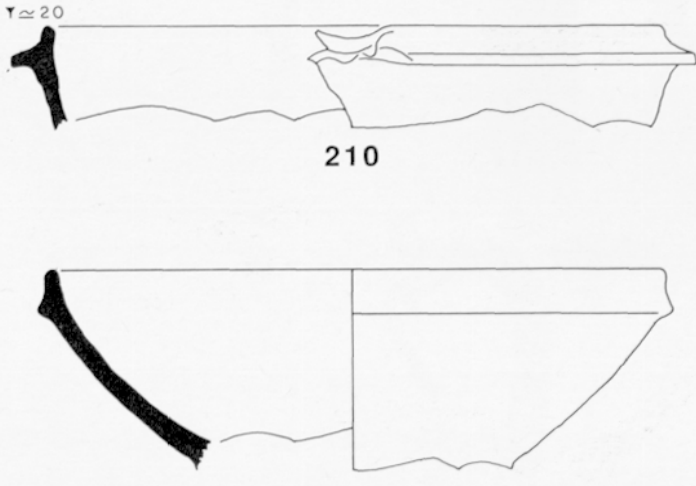

211

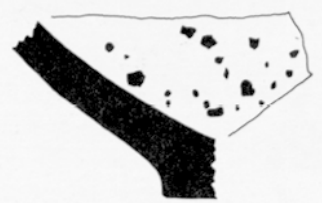

212
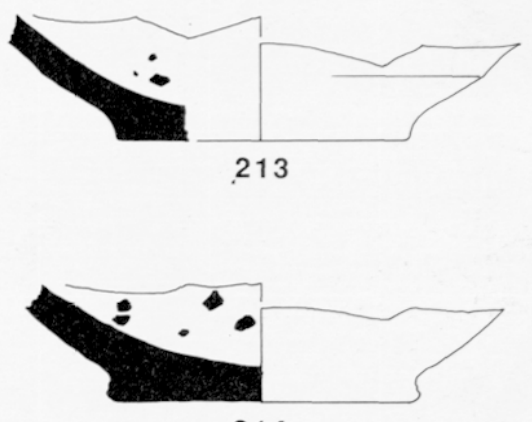

214
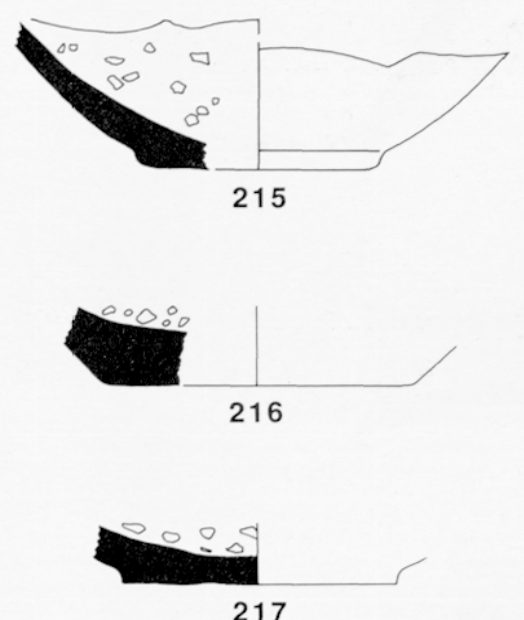

trouvés à Apt. Des caractéristiques évidentes révèlent un groupe inédit.

L'impression qui s'en dégage est d'être en présence de tentatives de "faire comme" des ateliers évolués sans avoir la capacité d'atteindre les mêmes qualités technologiques dans le façonnage et les traitements de surface ou esthétiques dans l'ornementation. Le souci de décorer est évident, même s'il se limite à tracer quelques stries concentriques sur un fond d'assiette, et que dire de ces empreintes sur un 

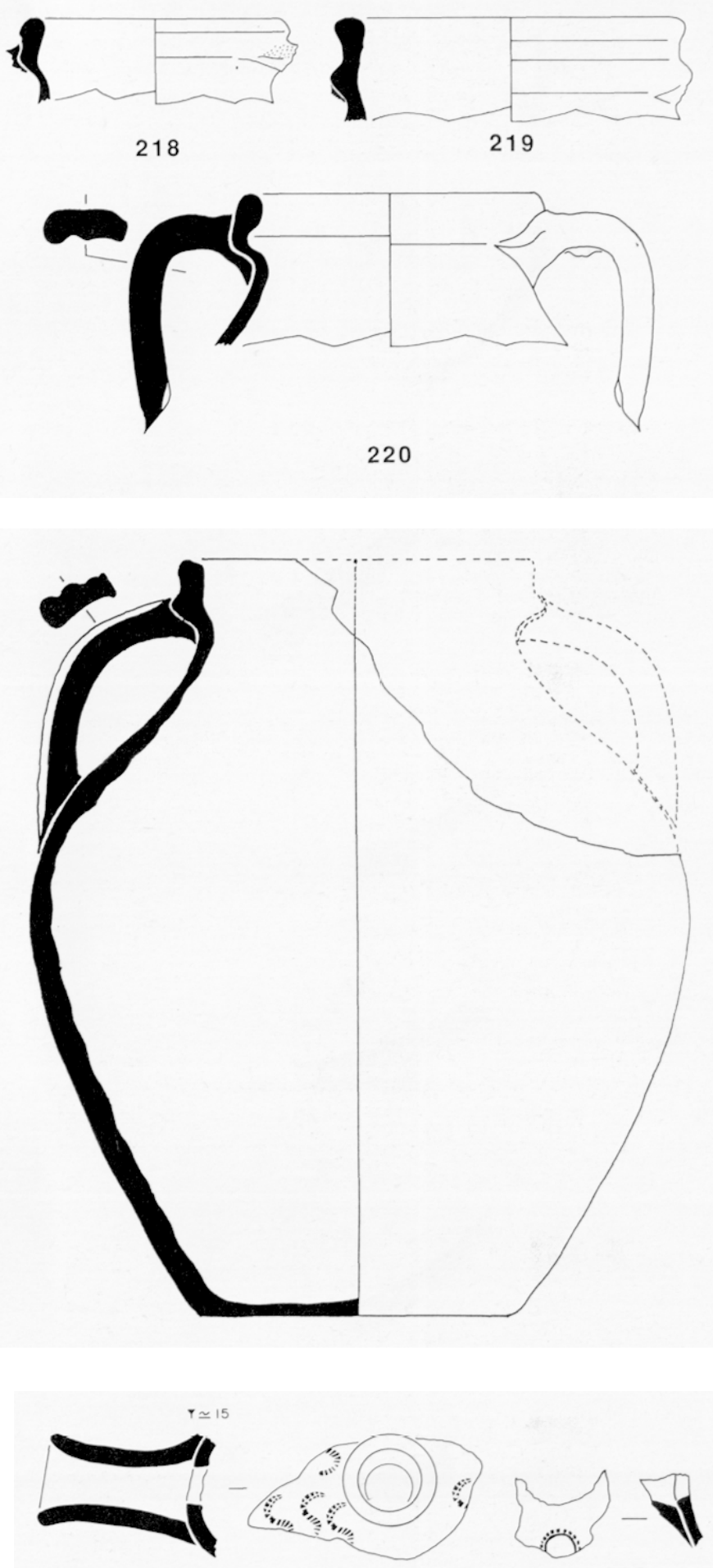

221
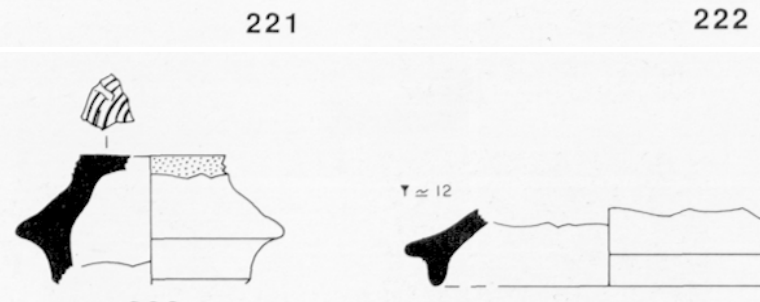

223
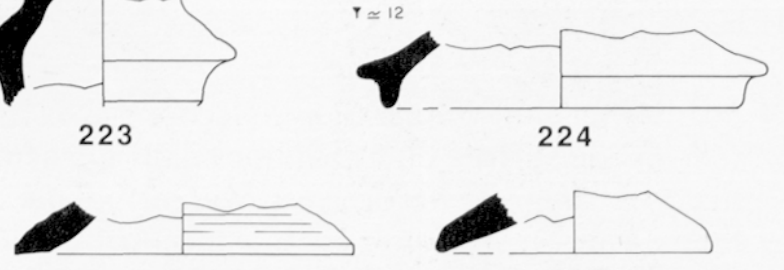

225

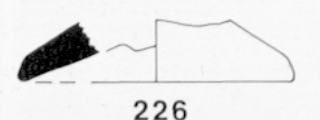

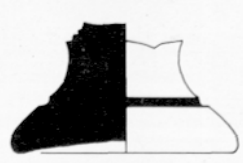

227

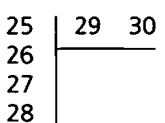

$\square 25$ DS.P. Forme 63. Petite amphore à lèvre en baïonnette généralement bien représentée [éch. 1/3].

$\square 26$ DS.P. Forme 63. Exemplaire provenant de Saint-Jean-deGarguier

(dessin aimablement communiqué par J.-B. Féraud) [éch. 1/3].

27 DS.P. Becs tubulaires [éch. 1/3].

28 DS.P. Couvercles [éch. 1/3].

129 DS.P. Pieds hauts [éch. 1/3].

130 DS.P. Fragment de tube cylindrique. Peut-être un manche de poêlon... [éch. 1/3].

marli faites avec cet objet que l'on n'a même pas pris le soin de façonner (fig. 17, $\mathrm{n}^{\circ}$ 94). Il est tentant de laisser aller son imagination avec le poinçon fissuré de la double rouelle et de trouver simpliste et banal d'y voir un outil toujours utilisé malgré son usure dans un atelier sur le déclin... Ne peut-on imaginer l'histoire d'un apprenti allant tenter sa chance en montant son propre atelier dans un village des environs ? Ou celle d'un ouvrier peu compétent, chassé par son patron qui lui laisse emporter des rebuts pour exercer ailleurs un métier qu'il maîtrisait mal ? L'un ou l'autre tentera cependant de le poursuivre avec les mêmes notions, comme en témoigne la décoration élaborée à laquelle participe ce motif. Comme en témoignent aussi les nombreux guillochis toujours malhabiles et souvent involontaires. Les tournassins qui les ont réalisés n'étaient certes pas dirigés d'une main très ferme. En tout cas sans ce doigté indispensable à la réalisation de ces excisions aiguës et régulières qui se retrouvent dans la production marseillaise. Elles exigent, il est vrai, une grande virtuosité pour réaliser ces séries de stries parallèles qui courent autour des panses et le long des bords.

Si maladroit et négligent qu'il ait été, ce potier marque d'une croix l'ambiance de l'époque dans laquelle il vivait, justement qualifiée de paléochrétienne mais que la laïcisation du vocabulaire appelle aujourd'hui plus banalement Antiquité tardive. Cette empreinte et les chrismes moulés en verre rappellent ici la naissance de croyances qu'il n'est pas nécessaire de partager pour en reconnaître la réalité historique et dont l'iconographie des DS.P. apporte des témoignages de plus en plus nombreux. 


\subsubsection{Catalogue des décors}

- Non répertorié. Petite rouelle sommaire mal imprimée, formée d'un cercle épais. Sur le marli de forme $3, n^{\circ} 95$ (fig. 17).

- 4548. Rouelle composée d'environ 15 secteurs. Sur le fond $d^{\prime}$ assiette $n^{\circ} 24$ (fig. 11).

- 4236. Rouelle classique : 13 points autour d'un cercle. Sur le marli de forme $3, \mathrm{n}^{\circ} 90$ (fig. 17). Se retrouve à Sainte-Propice (B.-du-Rh.) avec les motifs 209, 2712, 4234 et 4235 sur une forme 23 à bec tubulaire (Boixadéra 1987).

- Non répertorié. Partie de rouelle de même construction que la précédente. Impression floue sur le fragment de marli $n^{\circ} 3$ (fig. 10).

- Non répertorié. Rouelle formée de cercles hachurés. Mal imprimée et peu lisible en raison de la corrosion de la surface. Sur le marli $\mathrm{n}^{\circ} 1$ (fig. 10).

- Non répertorié. Peut-être la même que la précédente. Sur le large marli de bol orangé $n^{\circ} 93$ (fig. 17).

- 16. Deux cercles de 15 et 24 pointillés en double rangée interrompue par une impression dans l'axe. Sur le grand marli de forme $1, n^{\circ} 1$ (fig. 31 ; hors fosse). Aussi en double rangée sur un marli de forme 1 à Marseille et, à Fréjus, sur un angle de marli découpé.

- 4555. Rouelle assez grande composée de deux cercles pointillés entourant une couronne de hachures. Sur la panse du bol de forme $6, n^{\circ} 112$ (fig. 19).

- 4559. Rouelle de gravure grossière. Sur le fond d'assiette à panse inclinée $n^{\circ} 61$ (fig. 14).
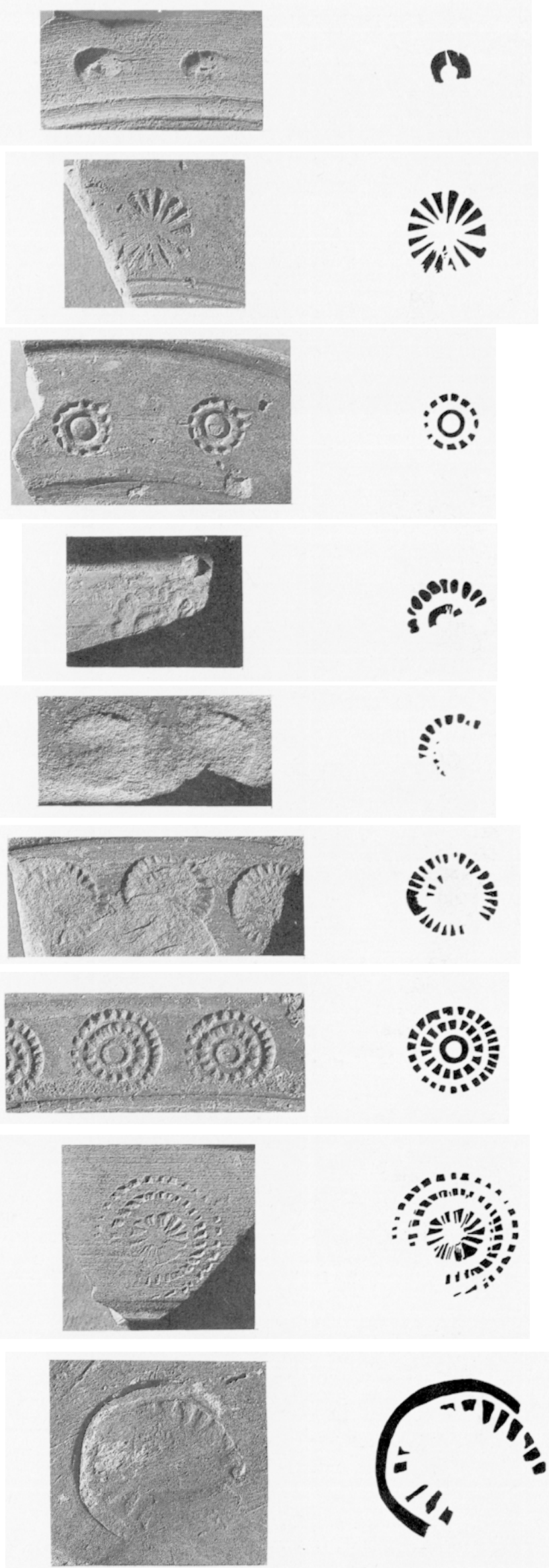

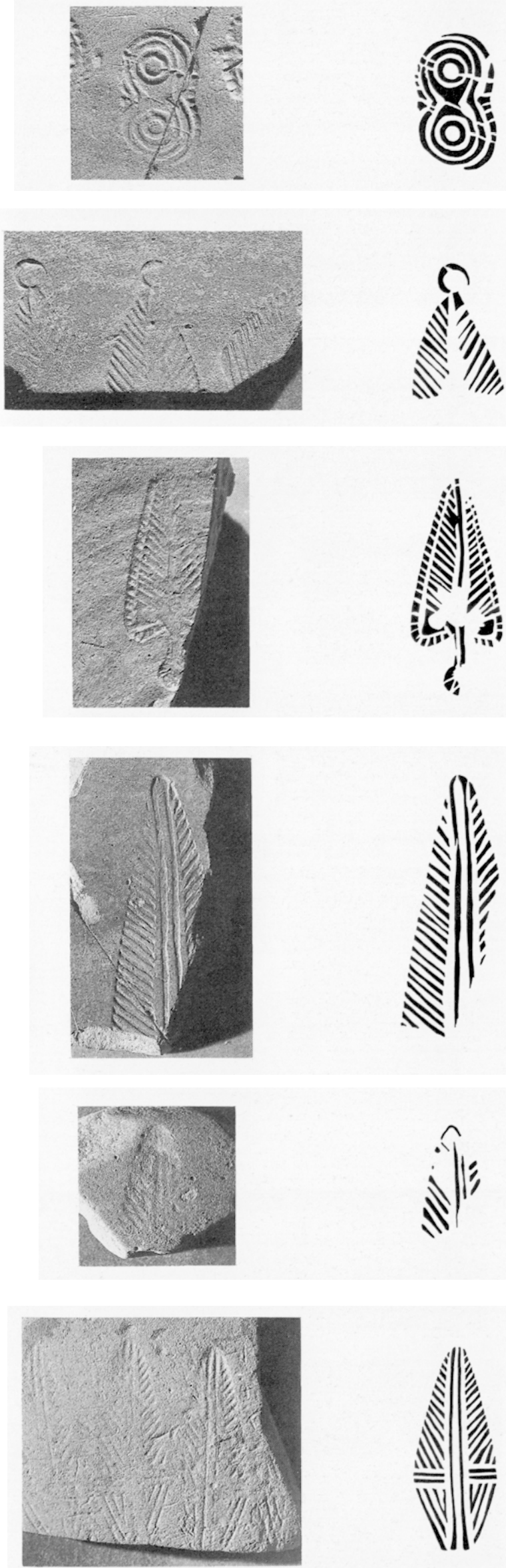

- 2015. Rouelle double. Poinçon fissuré. Avec l'arceau 4545 autour de la croix 4544, sur le fond d'assiette $n^{\circ} 27$ (fig. 11). Nombreux exemplaires intacts, donc plus anciens : dans les Bouches-du-Rhône, à Marseille Vieux-Quartiers sur 9 objets : 3 fonds de coupelles, un marli de forme 3, 3 panses de forme 15, une panse de forme 18 ; à La Bourse sur 6 pièces : 4 fonds d'assiettes et 2 de coupelles; 1 à Saint-Victor; 9 à Saint-Blaise dont un marli de forme 1 et un fond d'assiette ; 1 à Vernègues sur un fond d'assiette; 2 à Istres sur un fond de coupelle de forme 2 et une panse de forme 6. A Cimiez (Nice, Alpes-Maritimes), 1 sur une panse de forme 18.

- 4552. Haut de palmette à nervures divergentes, surmontée d'une rouelle. Sur le fond d'assiette orangé n ${ }^{\circ} 58$ (fig. 14).

- 4550. Palmette complexe à nervure centrale verticale et nervures latérales divergentes, entourée de pointillés. Pastille à la base qui se termine par un pédoncule portant une rouelle. Sur le fond de coupelle $n^{\circ} 86$ (fig. 16).

\begin{abstract}
- 4551. Haut de palmette à double rainure centrale verticale et nervures latérales divergentes. Sur le fragment de fond
\end{abstract} d'assiette $n^{\circ} 60$ (fig. 14).
- Non répertorié. Sommet de palmette à double rainure centrale verticale et nervures latérales divergentes. Peut-être un motif en losange. Sur la panse de forme $18 n^{\circ} 139$ (fig. 20).

- 2340. Palmette en losange à doubles nervures centrales, verticales et horizontales ; nervures divergentes dans la partie basse comme dans la partie haute. De l'atelier dit "de SaintJulien" (Rigoir 1985). Sur la panse de forme 6 orangée, $n^{\circ} 110$ (fig. 19). A Saint-Julien-les-Martigues (B.-du-Rh.), sur 9 pièces : 1 fond de forme 1,7 panses de bols dont une forme 6 , deux formes 15 et une forme 18 ; à Marseille Vieux-Quartiers, sur deux panses de forme 6 dont une orangée et sur un fond d'assiette ; à Pélissanne (B.-du-Rh.), sur une panse de forme 6 ; à Nice (A.-M.), sur une panse de bol et à Aix-enProvence (B.-du-Rh.) sur une panse de forme 6. 
- 3421. Palmcttc complexe. Nervure centrale verticale unique mais nervures latérales divergentes à la base et convergentes dans la partie haute. Entourée d'une ligne continue puis de pointillés. Rouelle à la base. Sur le fragment de fond d'assiette $n^{\circ} 25$ (fig. 11). Aussi à Saint-Blaise sur un fond d'assiette.

- 4561. Petit arceau simplement formé d'une ligne pointillée. Mal imprimé, la partie concave tournée vers le centre du fond, probablement d'assiette, $n^{\circ} 22$ (fig. 11).

- 4553. Arceau franchement imprimé sur la partie verticale du bol caréné $n^{\circ} 130$ (fig. 20) et sur la forme à bec tubulaire $n^{\circ} 222$ (fig. 27).

- 4545. Arceau plus ouvert participant au décor du fond $d^{\prime}$ 'assiette $n^{\circ} 27$ (fig. 11) en alternance avec la rouelle double 2015 autour de la croix 4544.

- Non répertorié. Partie de colonnette. Poinçon usé imprimé sur la panse de forme $18, n^{\circ} 140$ (fig. 20).

- 4549. Longue colonnette composée comme la précédente d'une ligne continue centrale flanquée de pointillés. Imprimée irrégulièrement de façon à en écarter ou resserrer les éléments. Compose des zigzags sur la panse de forme $6, \mathrm{n}^{\circ} 108$ (fig. 19).

- 4557. Colonnette composée de deux traits parallèles entourés de trois côtés de pointillés. Sur le fragment de panse (de vase fermé ?) $n^{\circ} 164$ (fig. 21).
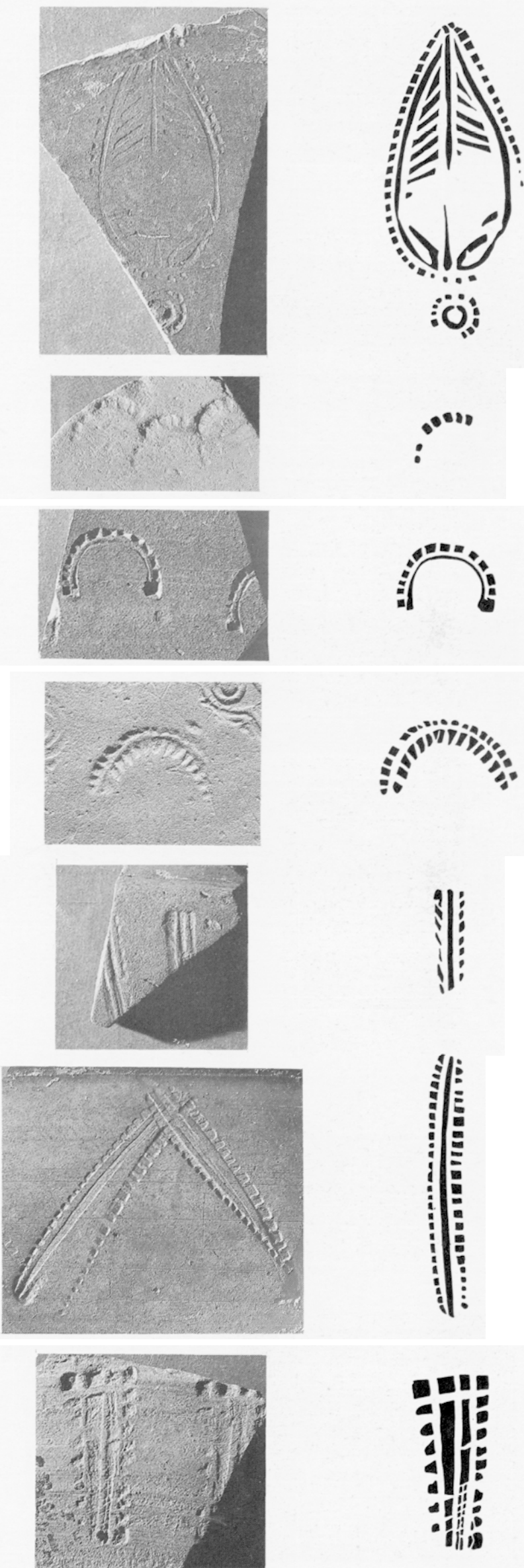


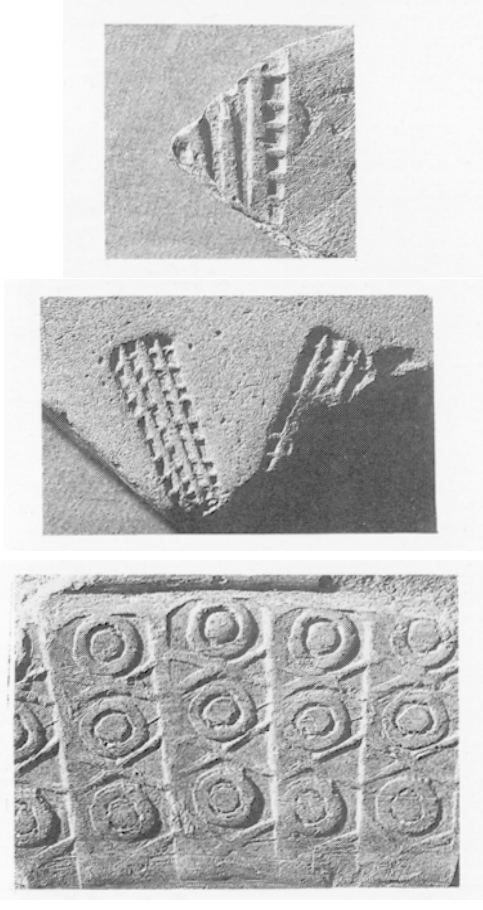
- 4560. Partie de colonnette. Fragment de même composition
que le précédent imprimé sur le fond d'assiette ${ }^{\circ} 21$ (fig. 11).
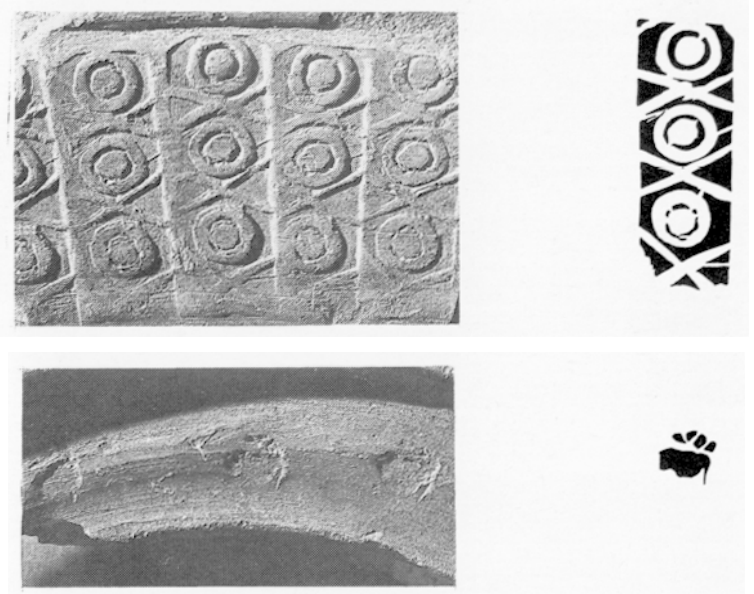

- 4554. Colonnette composée de quatre lignes parallèles pointillées découpées en oblique. En étoile sur le fond

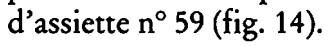

- 4442. Belle composition rectangulaire d'au moins trois rouelles séparées par des croisillons. Sur le marli ${ }^{\circ} 2$ (fig. 10) et le fragment de marli $n^{\circ} 92$ (fig. 17).
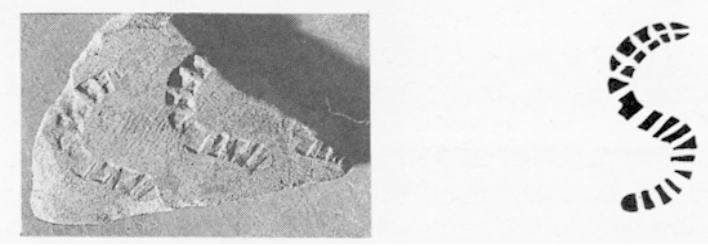

- 4558. Petit motif imprécis, imprimé au moyen d'un outil indéterminé qui ne paraît pas avoir été gravé en vue de cet usage. Sur le marli de forme $3, n^{\circ} 94$ (fig. 17).

- 4556. Motif en “S” imprimé sur la panse de la forme à bec tubulaire $n^{\circ} 221$ (fig. 27).

- 4547. Autre " $S$ " plus complexe dont quatre impressions accompagnent le motif suivant sur le fond d'assiette $n^{\circ} 57$ (fig. 14).
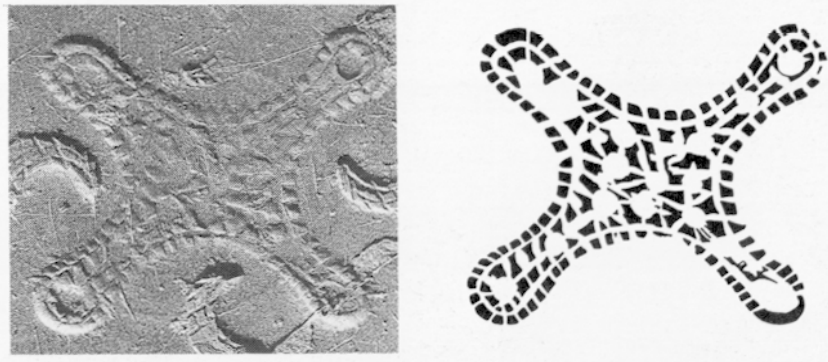

- 4546. Motif très complexe mais de gravure négligée : croix (?) entourée de pointillés, remplie de pastilles disposées à chaque extrémité des branches et en trois lignes de trois au centre, reliées par une résille de traits inorganisés. Avec le précédent.
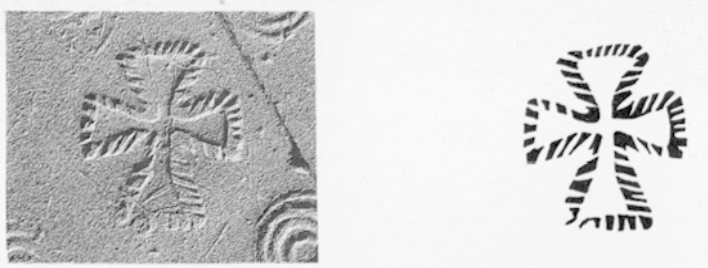

- 4544. Croix latine dessinée d'une ligne hachurée. Gravure libre. Au centre du fond d'assiette $n^{\circ} 27$ (fig. 11), avec la double rouelle 2015 et l'arceau 4545 . Cet exemplaire porte à 16 le nombre de poinçons répertoriés qui figurent une croix ou dont le principal élément est la croix. 

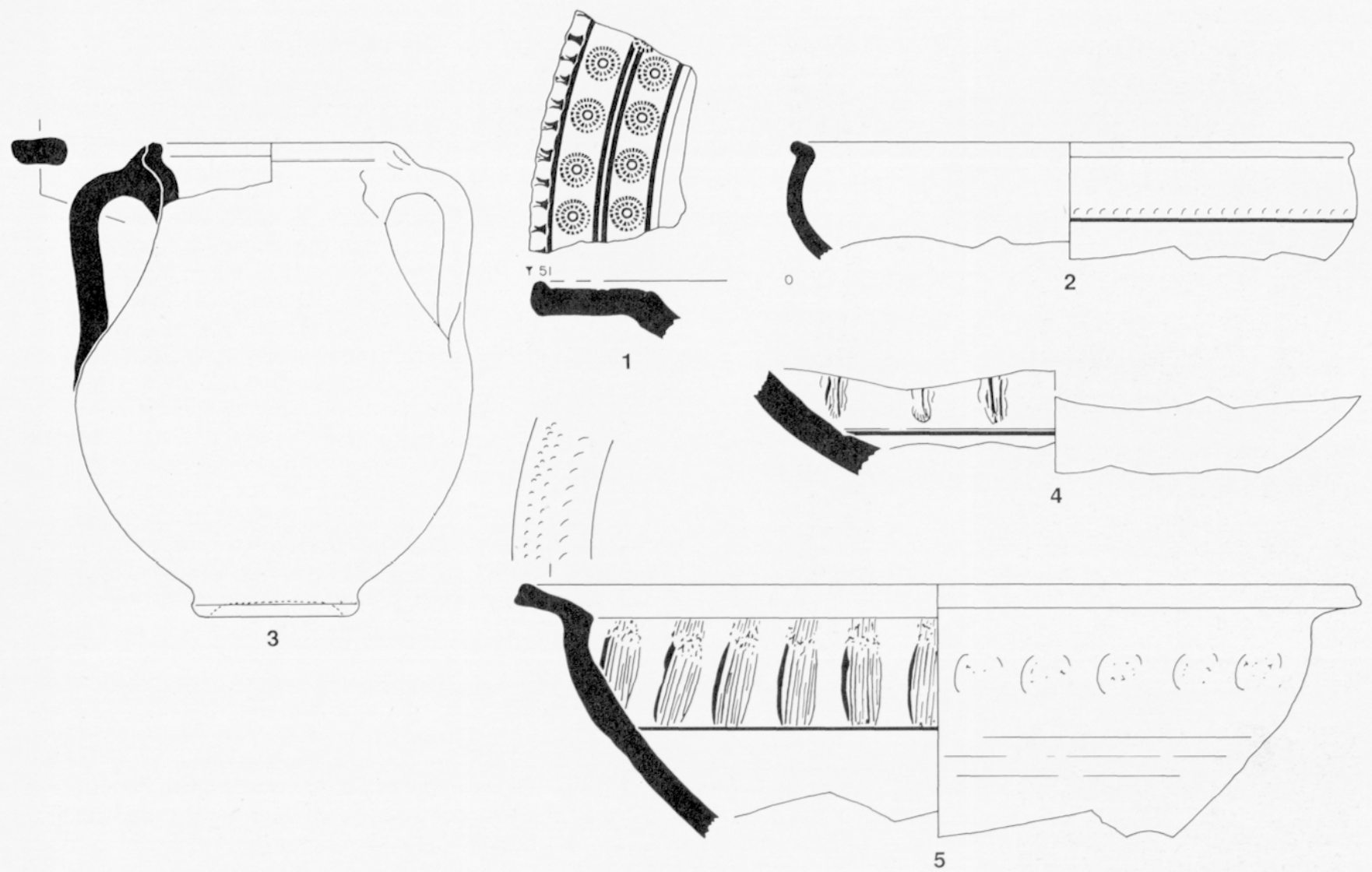

131 DS.P. hors fosse. $N^{\circ} 1$ : forme 1 ; grand marli décoré de godrons et de la rouelle 16. Décor non continu : sur le tesson sont visibles huit impressions sur deux rangs puis une dans l'axe. Ce fragment montre le danger des reconstitutions : une cassure à un centimètre près aurait laissé présumer d'un décor répétitif. $N^{\circ} 2$ : forme 6 ; orangée, de profil languedocien. Traces de guillochis involontaires au-dessus d'une rainure. $\mathrm{N}^{\circ} 3$ : forme 59 ; cuite partiellement en oxydation, cette pièce est une bonne illustration de cette forme, plus grande et plus pansue que celle de Saint-Julien (Rigoir 1987, 195). Contenance : 1,57 I. N $N^{\circ} 4$ et 5 : forme $3 c$; le $n^{\circ} 4$ est très grossier ; il semble que les empreintes aient été faites avec l'extrémité du doigt, des stries plus profondes ayant pu être marquées par l'ongle. Le $n^{\circ} 5$ est soigné, son marli est décoré de guillochis très légers. On distingue à l'extérieur les empreintes des doigts qui maintenaient la paroi pendant que l'autre main formait les dépressions [éch. 1/3].

$\square 32$ Formes $3 c$ retrouvées hors de la fosse ; dessinées figure 31. Si le travail de l'argile, surtout au tour, est réputé pour adoucir la peau, il ne semble pas que ce soit l'état des doigts qui ont tracé ces creux où ils ont laissé des stries bien marquées [sans éch.].
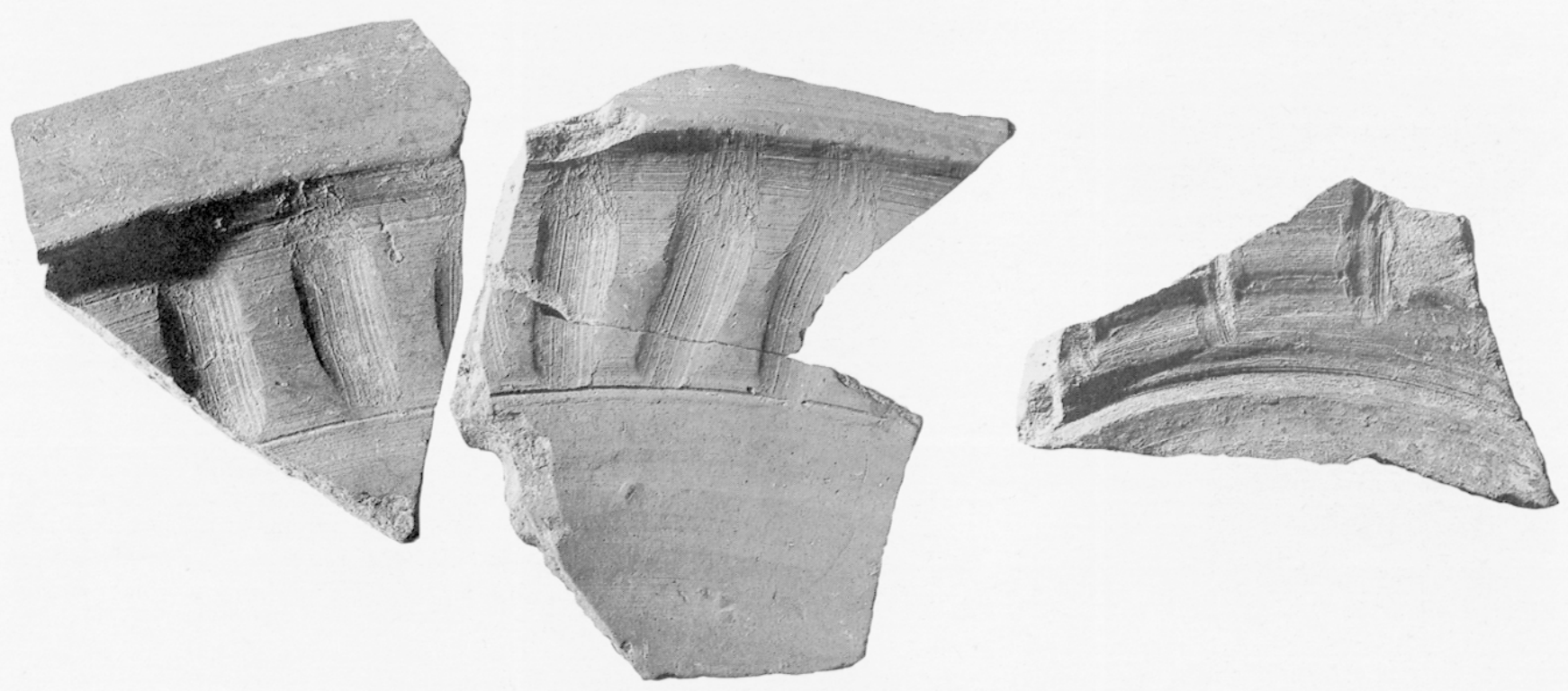


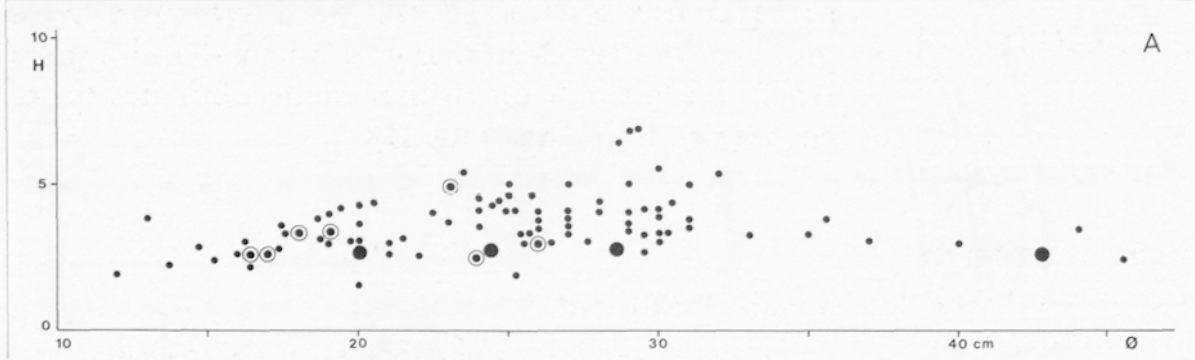

\section{3}

Corrélations des mesures profondeur/diamètre effectuées sur les DS.P. En abscisses les diamètres, en ordonnées les profondeurs. Les cotes sont relevées à l'intérieur des vases, la présence de marlis perturbant notablement les comparaisons avec les autres formes ; la largeur de ces bords peut en effet atteindre le tiers du diamètre de certains bols. Pour deux types de formes, on obtient des nuages d'orientation différentes. Diagramme $\boldsymbol{A}$ : les assiettes, toutes formes confondues. Grosses pastilles noires : les assiettes plates de Gardanne ; cercles pointés : assiettes creuses du même site ; petits points : assiettes provenant d'autres sites mais toujours de la production provençale. Les points s'étalent autour d'une horizontale à $3 / 4 \mathrm{~cm}$ en moyenne, la fourchette la plus large correspondant aux largeurs les plus fréquentes. Cette constante s'explique par le fait de la construction même de la forme plate "assiette". Diagramme B : les bols, toutes formes confondues. Grosses pastilles noires : les bols de Gardanne ; petits points : bols provenant d'autres sites. Un axe moyen incliné d'environ $35^{\circ}$ par rapport à l'horizontale peut être tracé. Le rapport hauteur/argeur demeure donc constant pour ces récipients aux formes continues : issues sur le tour de la masse de la pâte, le profil monte et s'évase suivant une courbe spontanée dont seuls les accidents (carène, lèvre) sont consciemment maîtrisés.

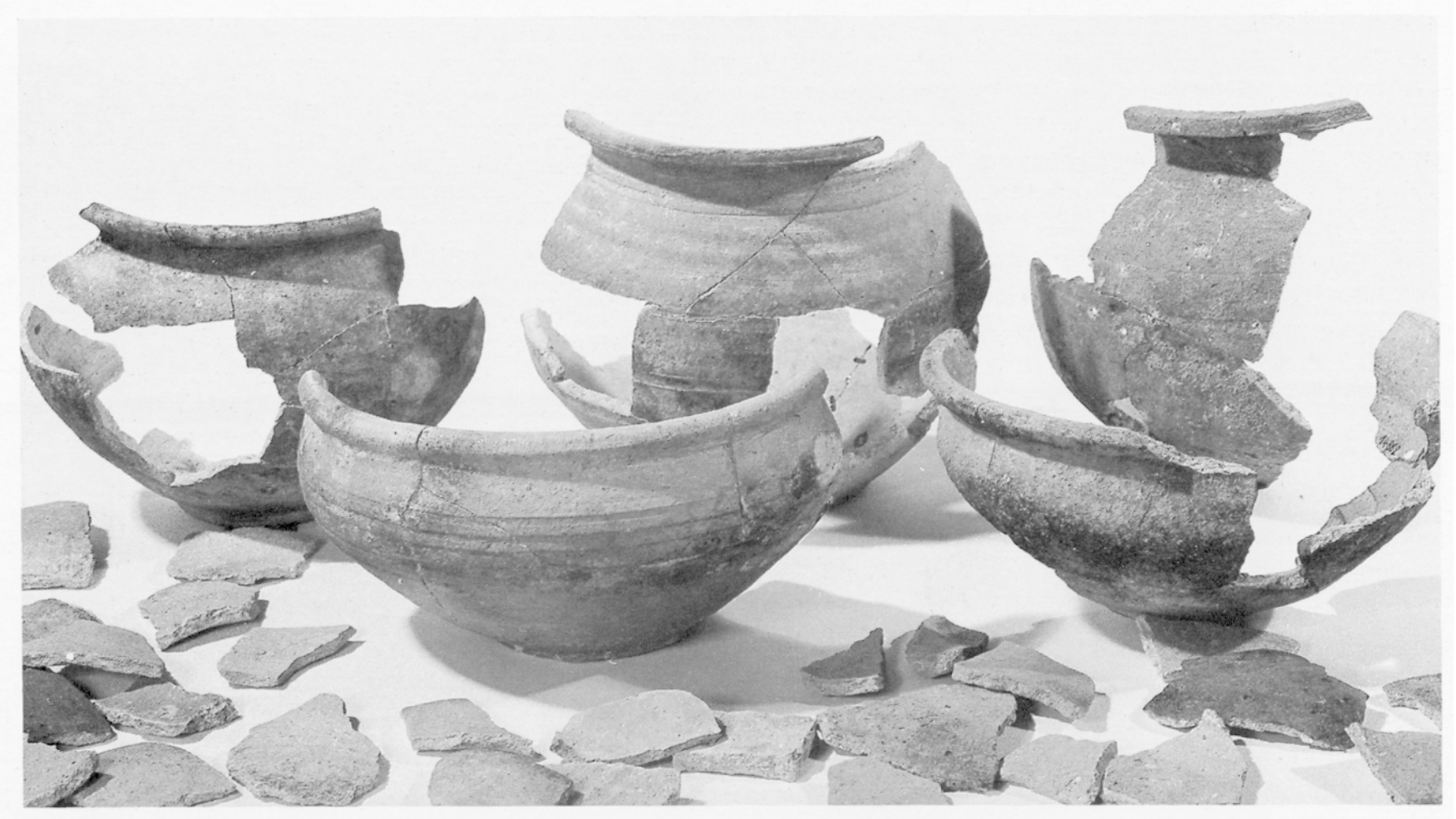

A Un exemple des formes de céramique commune grise reconstituées. 


\subsection{LA CERAMIQUE COMMUNE GRISE}

\subsubsection{Introduction au catalogue des formes}

Le catalogue des céramiques communes grises, en pâte siliceuse cuite en atmosphère réductrice, utilise la typologie élaborée à partir des premières études réalisées sur le matériel des sites de Marseille, Aix-en-Provence, $\mathrm{La}$ Gayole et Rougiers. Présentée lors du $3^{\mathrm{e}}$ Congrès international de céramologie de Sienne en 1984 (C.A.T.H.M.A. 1986, 45-48) et complétée par de nouvelles séries de céramiques du Vaucluse (Kauffmann 1987, 74-78) et des Bouches-du-Rhône (Boixadéra 1987, 102-110), cette typologie s'est ensuite considérablement enrichie avec la variété du répertoire fourni par le site de Saint-Blaise.

Aujourd'hui, les découvertes de Gardanne permettent d'élargir encore cette nomenclature des poteries communes dont les faciès micro-régionaux reflètent la multiplicité des ateliers producteurs.

La répétition de nouveaux éléments bien marqués nous a contraints à adjoindre dans certains cas une lettre ou un chiffre aux catégories définies dans un schéma de base ouvert sans cesse au renouvellement de la documentation.

La céramique commune grise de Gardanne, tournée rapidement et en grandes séries, offre des ensembles homogènes, même s'il est parfois difficile d'attribuer tel ou tel rebord à un type précis. La quasi-uniformité technologique des lèvres et des profils rencontrés en grand nombre sur le site de NotreDame plaiderait en faveur d'une production issue d'un ou plusieurs ateliers, dans une aire géographique restreinte. Cette nouvelle piste nous inciterait à parler désormais de "céramiques communes grises provençales", ou plus précisément "de la région marseillaise", malgré l'apparente uniformité qui semblait caractériser à première vue ce groupe régional au sens large du terme (C.A.T.H.M.A. 1986, 50).

\subsubsection{Formes $A$}

Ce sont des formes fermées (ollae), plutôt globulaires, rarement munies d'anses, avec un diamètre maximum situé à mi-hauteur et un fond plat étroit. Elles ne sont pratiquement jamais décorées : tout au plus la panse est-elle parfois animée de quelques raies de tournage ou d'incisions horizontales plus marquées, mais ces exemples restent très rares (fig. 37, $\mathrm{n}^{\circ} 4$ et 7 ; fig. $39, \mathrm{n}^{\circ} 2$ ).

La typologie des formes A ne concerne que les différents types de rebords. Le chiffre distingue les groupes principaux, complété de la lettre A pour la présence d'anses (ex. : A2A) ou d'une autre lettre précisant des séries secondaires mieux définies (ex. : A3T). La lettre A est utilisée seule pour des types encore peu fréquents (tabl. II).

\section{CATALOGUE}

\section{- Forme A1 (fig. 34)}

Caractères : rebord de section très simple, légèrement déversé, d'épaisseur comparable à celle de la panse. La section de la lèvre peut être anguleuse (par ex. $n^{\circ} 8$ à 9,11 à 15), ou arrondie ( $n^{\circ} 5$ et 6$)$, ou former un petit bourrelet ( $n^{\circ} 2$ à 4$)$. Il n'y a pratiquement pas de col (sauf peut-être sur les $n^{\circ} 10$ et 13 ) : la liaison bord-panse est anguleuse (par ex. $\left.n^{\circ} 2,11,12,15\right)$ ou arrondie $\left(n^{\circ} 4,5,9 \ldots\right)$.

\section{- Forme A2 (fig. 35 ; 36 ; 37)}

Caractères: les rebords comportent toujours une gorge interne plus ou moins accentuée qui les différencie du type A1. Les autres caractères sont comparables : la forme est simple, la section de la lèvre anguleuse avec arête externe marquée, ou arrondie, ou en bourrelet. L'épaisseur du bord est comparable à celle de la panse ou un peu plus importante. Le col est peu marqué ou inexistant.

Mises à part quelques variantes isolées (fig. $35, \mathrm{n}^{\circ} 1$ à 8 ), la plupart des éléments sont à rattacher à une série que l'on qualifiera de "locale", étant donné leur grand nombre dans la fosse. Cette série homogène présente malgré tout des nuances de détail :

- fig. 36, n ${ }^{\circ} 1$ à 6 : rebord déversé, formant avec la panse un angle voisin de $90^{\circ}$, et absence de col ;

- fig. $36, n^{\circ} 7$ à 9 : lèvre plus verticale, la liaison bord-panse est plus arrondie;

- fig. $37, \mathrm{n}^{\circ} 1$ à 7 : la lèvre est assez épaisse ( 8 à $9 \mathrm{~mm}$ ), avec l'arête externe marquée.

\section{- Forme A2A (fig. 38)}

Une dizaine d'objets au rebord de type A2 sont munis de deux anses $\left(n^{\circ} 1\right.$ à 5$)$, à la section plus ou moins aplatie. La fixation exécutée rapidement contre le rebord entraîne parfois des déformations $\left(n^{\circ} 1\right)$.

\section{- Forme A3 (fig. 39 et 40)}

Caractères : les rebords ont une section à peu près carrée, plus épaisse que la panse. Le replat supérieur est horizontal ou légèrement incliné vers l'intérieur. L'arête externe, marquée, est à peu près verticale (fig. $39, \mathrm{n}^{\circ} 1$ à 12 ).

Une variation de la technique de façonnage peut aboutir à des sections légèrement différentes, parfois triangulaires (forme A3T ; fig. 40, $n^{\circ} 1$ et 2), ou aux arêtes émoussées (forme $A 3$, variantes; fig. $40, \mathrm{n}^{\circ} 3$ à 6 ).

Les formes $\mathrm{A} 3$ constituent elles aussi un groupe important et assez homogène parmi lequel on dénombre 10 formes $\mathrm{A} 3 \mathrm{~T}$.

\begin{tabular}{|l|c|c|c|c|c|c|c|}
\hline Formes & A1 & A2 & A2A & A3 & A4 & A div. & Total \\
\hline NMI & 24 & 88 & 10 & 64 & 15 & 18 & 219 ex. \\
\hline$\%$ & 11 & 40,2 & 4,5 & 29,2 & 6,8 & 8,2 & $100 \%$ \\
\hline
\end{tabular}

Tabl. II Formes A : répartition par variantes. 


$\begin{array}{ll}3 & 3 \\ 1 & 3 \\ 1 & 3 \\ 1 & 3 \\ 1 & 3 \\ 1 & 1\end{array}$



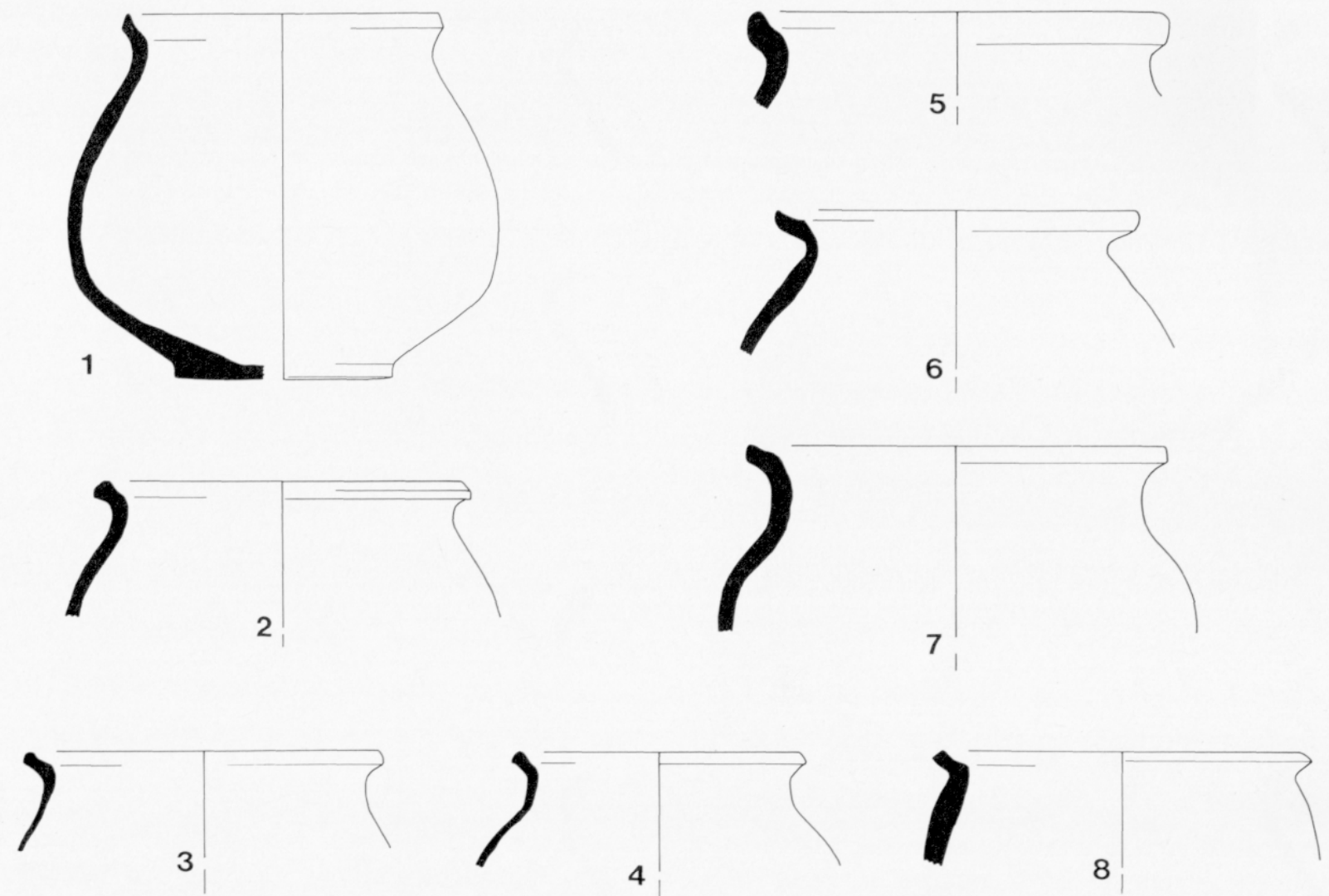

I 35 Commune grise. Forme A2, variantes isolées [éch. 1/3].

avec les marmites médiévales, de forme très pansue ou globulaire, comportant plusieurs anses.

- Formes A, types isolés (fig. 42)

Dix-huit objets de forme A ne peuvent être rattachés directement à une des séries précédemment décrites. Six sont façonnés avec une gorge interne très marquée $\left(\mathrm{n}^{\circ} 1\right)$. Cinq comportent un bord très simple, allongé, presque vertical $\left(n^{\circ} 2\right)$. Les autres sont plus ou moins proches des types $\mathrm{A} 4\left(\mathrm{n}^{\circ} 3\right.$ et 4$)$, ou A3T ( $n^{\circ} 5$ et 6$)$, ou alors exceptionnels $\left(n^{\circ} 7,8\right.$ et 9$)$.

\section{LES DIMENSIONS ET LEURS RAPPORTS (tabl. III)}

\section{- Diamètres des bords}

- Type A1 (fig. 34) : compris entre $9 \mathrm{~cm}$ pour les objets les plus petits et $15 \mathrm{~cm}$ pour les plus gros, la plupart (environ les trois quarts) étant compris entre 12 et $14 \mathrm{~cm}$.

- Types A2 divers (fig. 35) : compris entre 10,5 et $15 \mathrm{~cm}$.

$\mathrm{La}$ distinction entre ces deux catégories (présence d'une gorge interne) n'est pas toujours évidente $:$ il s'agit avant tout de bords aux formes très simples. Les quelques exemplaires A2 divers de la figure 35 ne sont que des variantes isolées. On constate dans les deux cas des objets aux dimensions bien différentes.

- Types A2 “locaux” (fig. 36 et 37) : par contre, dans cette catégorie la plus importante numériquement, on constate que les diamètres des bords sont beaucoup plus standardisés; ils sont compris entre 13 et $14 \mathrm{~cm}$ pour les objets à lèvre mince (fig. 36) à l'exception du $n^{\circ} 2$ pour lequel une ovalisation peut être évoquée. Les objets à lèvre plus épaisse (fig. 37) ont un diamètre compris entre 14 et $15 \mathrm{~cm}$. Parmi les formes à anses

\begin{tabular}{|l|c|c|c|c|c|c|c|}
\hline Formes & fig./ $\mathbf{n}^{\circ}$ & $\begin{array}{c}\text { diam. bord } \\
\text { (en } \mathrm{cm})\end{array}$ & $\begin{array}{c}\text { diam. max. } \\
\text { (en } \mathrm{cm})\end{array}$ & $\begin{array}{c}\text { diam. fond } \\
\text { (en cm) }\end{array}$ & $\begin{array}{c}\text { hauteur } \\
\text { (en cm) }\end{array}$ & $\begin{array}{c}\text { angle fond } \\
\text { (en degrés) }\end{array}$ & $\begin{array}{c}\text { contenance } \\
\text { (en litres) }\end{array}$ \\
\hline A2 & $35 / 1$ & 11,5 & 15,5 & 8 & 13 & $35-40$ & 1,4 \\
A2 & $36 / 1$ & 13 & 17 & 8 & 15,5 & 40 & 2 \\
A2 & $37 / 1$ & 15 & 19 & 7,5 & 16,5 & $35-40$ & 2,5 \\
A2A & $38 / 4$ & 14 & 19 & $?$ & $17 ?$ & $?$ & $2,9 ?$ \\
A3 & $39 / 1$ & 16 & 19 & 8 & 15,5 & 30 & 2,7 \\
A3 & $39 / 5$ & 13,5 & 16,5 & 8 & 14,5 & 45 & 1,7 \\
A3 & $40 / 4$ & 12 & 15,5 & 6,5 & 12,5 & 40 & 1,3 \\
A4A & $41 / 2$ & 16 & 23,5 & 9 & 21 & $30-35$ & 5,2 \\
\hline
\end{tabular}

Tabl. III

Mesures des formes A complètes. 

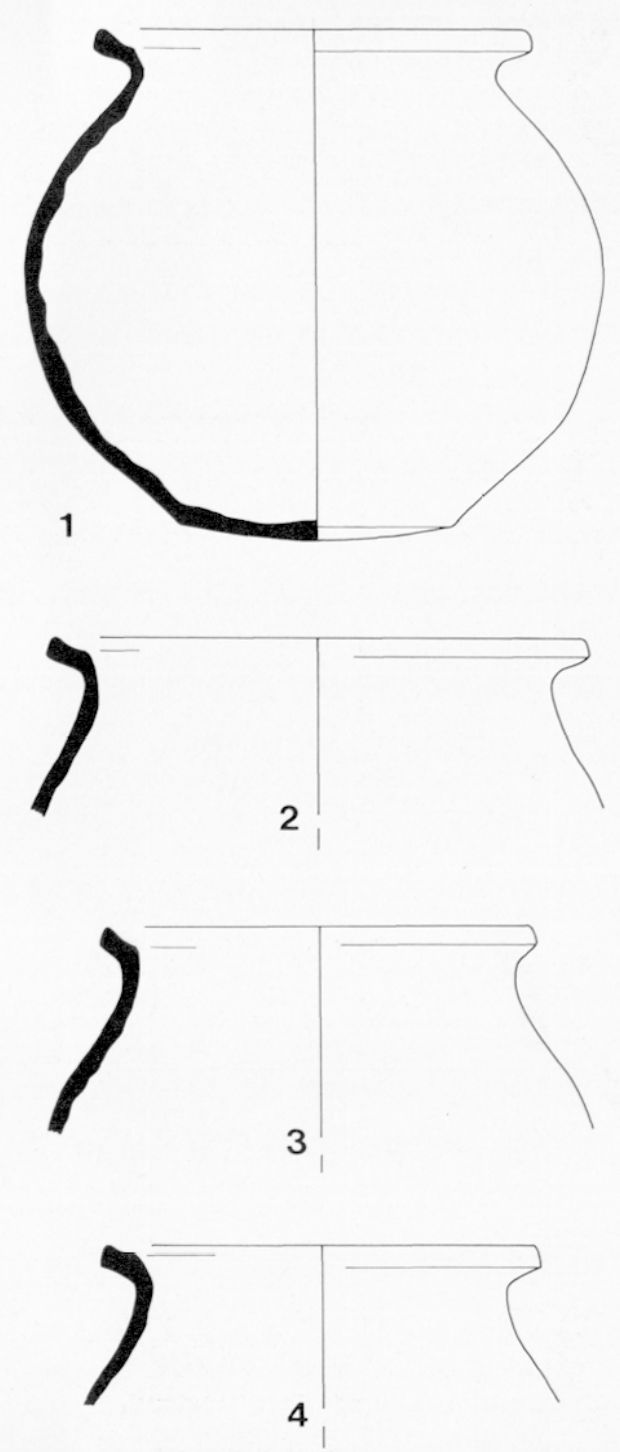

A2A (fig. 38), on retrouve ces mêmes dimensions sur les objets à rebord de type "local".

- Types A3 et A3T (fig. 39 et 40)) : compris entre 12 et $16 \mathrm{~cm}$.

- Types A4 et A4A (fig. 41) : compris entre 13 et $17 \mathrm{~cm}$.

- Types A diverses rares (fig. 42) : compris entre 12 et $15 \mathrm{~cm}$.

- Rapport entre le diamètre des bords et le diamètre maximum de la panse (fig. $43 \mathrm{~A}$ )

Les mesures effectuées sur 22 objets font apparaître un groupement assez homogène (à l'exception de la grosse marmite A4A). Le diamètre des bords est compris entre 12 et $15 \mathrm{~cm}$, le diamètre maximum entre 15 et $19 \mathrm{~cm}$, ce qui donne un rapport diamètre maximum/diamètre du bord compris entre 1,2 et 1,4 avec une moyenne à 1,3 . A l'inverse, on peut considérer le rapport diamètre du bord/diamètre maximum, soit une fourchette de 0,7 à 0,8 , avec une moyenne à 0,75 . L'approximation est ainsi de l'ordre de 7,5\%.

- Rapport entre le diamètre du bord et la hauteur (fig. 43 B)

Les mesures ne portent que sur 8 objets. La progression en fonction de leur taille est régulière. Le groupe d'objets de dimensions moyennes montre un rapport hauteur/diamètre du bord compris entre 0,96 et 1,2 (à l'inverse, diamètre/hauteur : 0,8 à 1). Seule la grosse marmite A4A se détache de ce
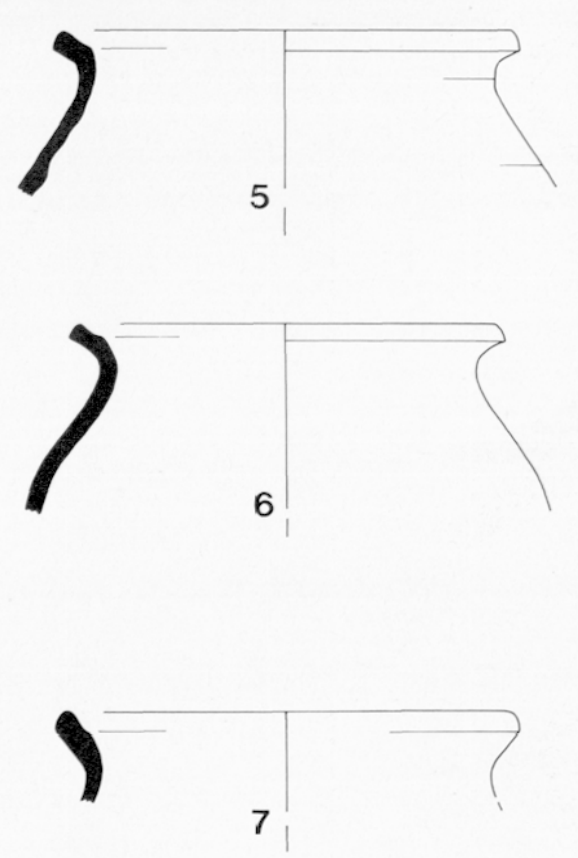

I 36

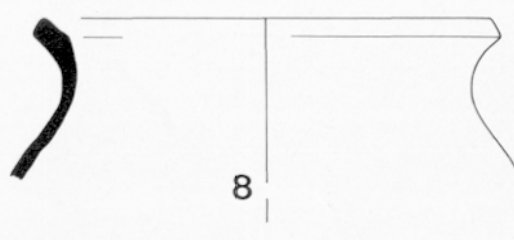

Commune grise. Forme $A 2$, série "locale" à rebord peu épais [éch. 1/3].

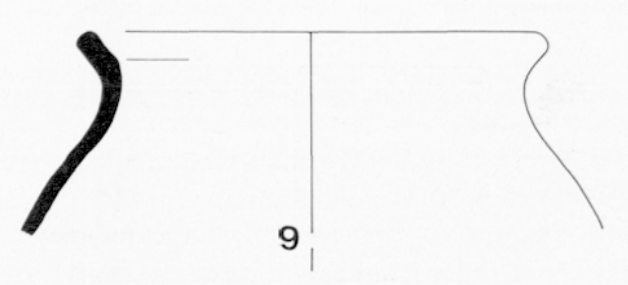

groupe (les rapports sont respectivement 1,3 et à l'inverse $0,76)$ : le diamètre du bord, dans ce cas, n'est pas proportionné à la taille de l'objet.

Sur le graphique figurent aussi des mesures de formes complètes déjà publiées attribuables à la même période et provenant de La Fourbine (Congès 1983), La Gayole (C.A.T.H.M.A. 1986, 47-48), Marseille-Bourse (Bonifay 1983), Saint-Blaise, Saint-Jean-de-Garguier (C.A.T.H.M.A. 1989 ; Foy 1990) et Velaux (Boixadéra 1987). La comparaison des proportions, bien que significative, est cependant moins évidente que pour les rapports entre le diamètre maximum et la hauteur.

- Rapport entre le diamètre maximum de la panse et la bauteur (fig. $43 \mathrm{C}$ )

On retrouve ici les mêmes phénomènes que ceux évoqués dans le paragraphe précédent. Mais la marmite A4A s'inscrit sur le même axe que les objets de taille moyenne. Les rapports diamètre/hauteur varient entre 1,1 et 1,3 avec une moyenne s'établissant vers 1,15 (à l'inverse le rapport hauteur/diamètre se situe entre 0,8 et 0,9$)$.

On peut constater que ces compléments s'organisent aussi suivant le même segment de droite incliné de $40^{\circ}$ à $45^{\circ}$ par rapport à l'horizontale. 


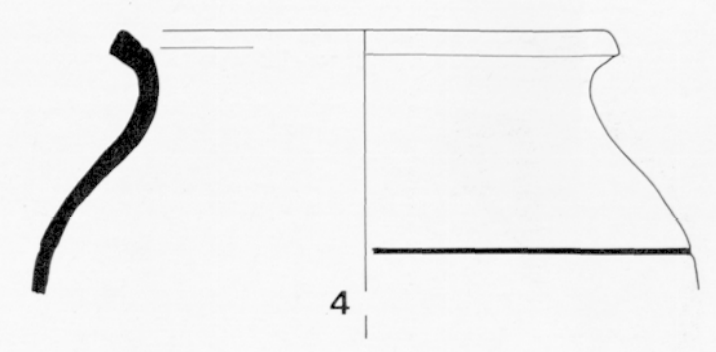

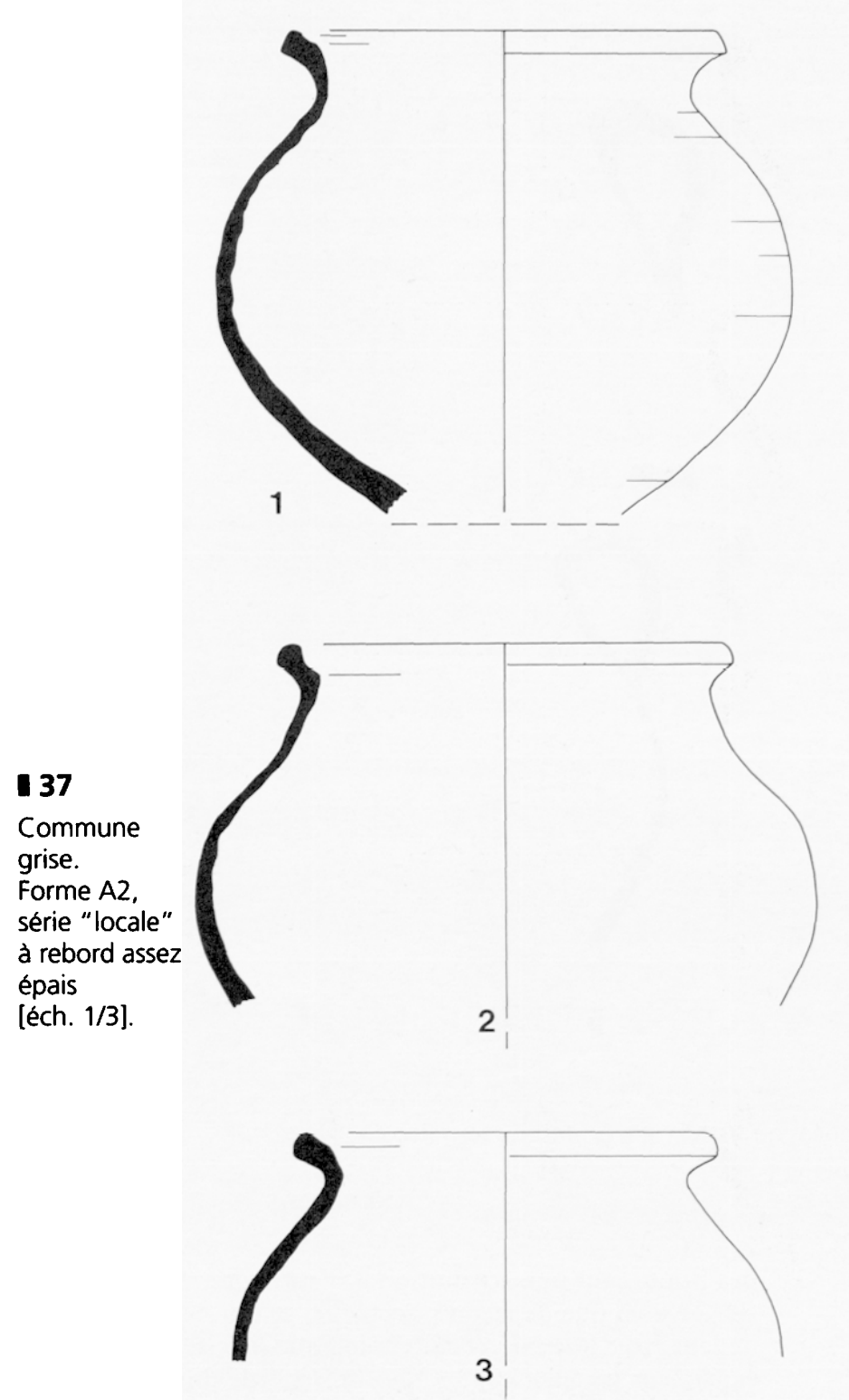

137

Commune grise.

Forme A2, série "locale" à rebord assez épais [éch. 1/3]

\section{- Position du diamètre maximum par rapport à la bauteur}

Le diamètre maximum est toujours situé à peu près à mihauteur, parfois un peu au-dessus, plus rarement un peu au-dessous, l'écart en pourcentage de la hauteur n'excédant pas $6 \%$.

- Mesures, proportions et types des fonds (voir chap. 2.5.4. et tabl. VI)

Une dizaine de fonds seulement peuvent être attribués avec une absolue certitude à des formes $\mathrm{A}$. Mais bien que leurs diamètres, compris entre 5 et $9 \mathrm{~cm}$, varient en fonction de la taille des objets, il ne semble pas que ce soit de façon particulièrement proportionnelle pour autant que l'on puisse en juger. Le rapport diamètre du fond/diamètre maximum varie entre 0,38 et 0,47 (à l'exception du $n^{\circ} 1$, fig. 35 , à la silhouette très lourde pour lequel ce coefficient atteint 0,51 ). On retrouve exactement le même écart, les mêmes chiffres, en comparant les mesures effectuées sur 14 formes A découvertes sur les sites déjà évoqués sans qu'il soit possible de préciser une valeur préférentielle; la moyenne calculée sur l'ensemble est de 0,4 .
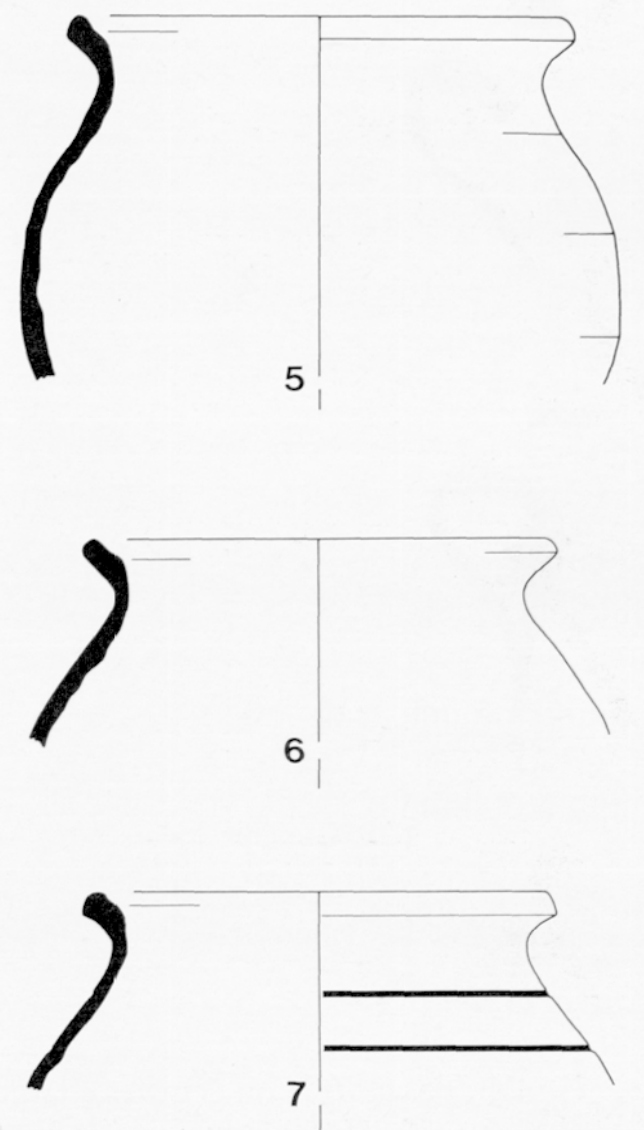

Le profil de ces fonds est indifféremment dégagé de la panse, avec un petit pied ou un petit bourrelet, ou très simple avec jonction directe ou continue entre la base de la panse et la face inférieure. La partie inférieure peut être plane, ou légèrement concave, le plus souvent convexe. L'angle compris entre la base de la panse et l'horizontale peut varier entre $30^{\circ}$ et $55^{\circ}$, bien que sa valeur la plus fréquente se situe autour de $40^{\circ}$.

Les fonds restent très comparables aux fonds des autres formes, B notamment, et il a semblé préférable, malgré ces quelques précisions, d'étudier un peu plus en détail les fonds dans leur ensemble (chap. 2.5.4.).

\section{- Les contenances (tabl. III)}

Les contenances mesurées sur les objets complets, vases ou marmites de taille moyenne et d'usage courant, les plus répandus, sont comprises entre 1,3 et 2,9 litres. Seule la grosse marmite A4A se détache de l'ensemble. Les objets les plus petits doivent avoir une contenance de trois quarts de litre environ. 


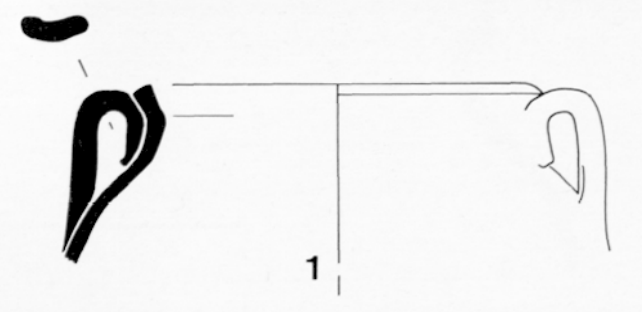

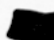

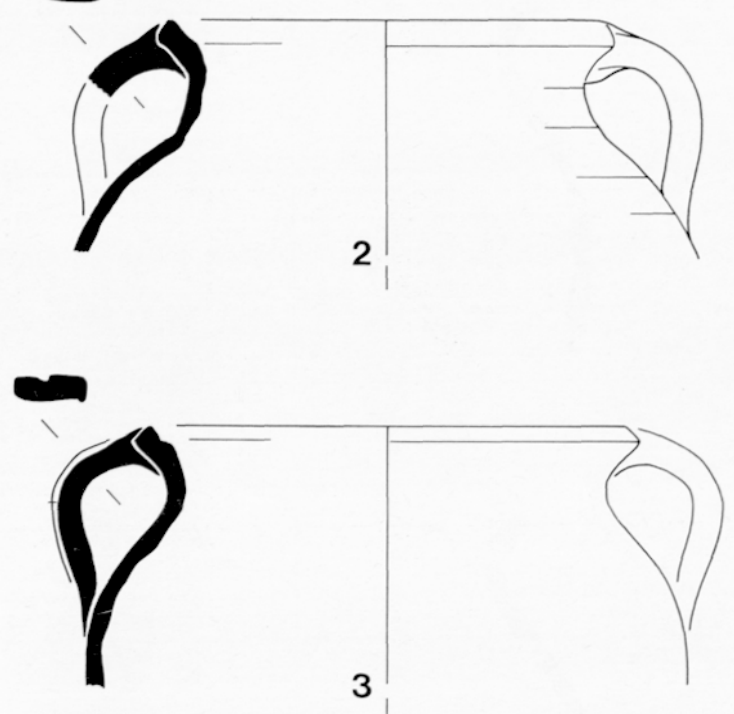

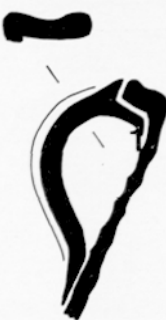

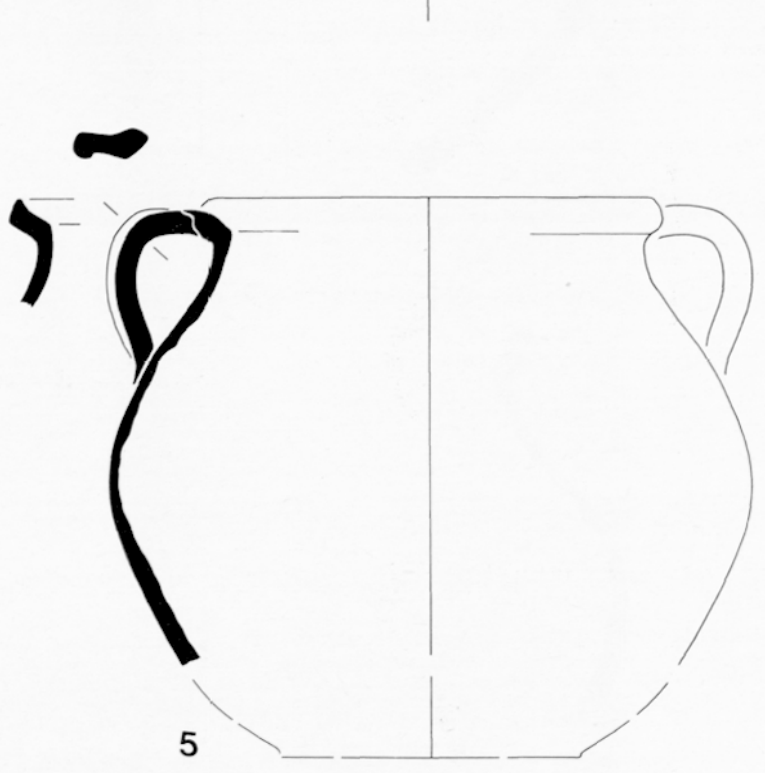

38 Commune grise. Forme A2A. No 1 : fabrication fruste ; 2 à 5 : fabrication "locale" [éch. 1/3].

\subsubsection{Formes $B$}

Ce sont des coupes ou écuelles à large ouverture, évasées, dont la hauteur avoisine la moitié du diamètre maximum. La panse présente souvent une carène ou un épaulement plus ou moins accentué, mais un certain nombre d'objets, à Gardanne, en sont dépourvus, ce qui leur confère une allure plus lourde, proche de celle des bols. Les fonds, étroits, semblent toujours être façonnés avec un petit pied (alors que sur les formes $\mathrm{A}$, on trouve indifféremment des fonds avec ou sans petit pied).

\section{CATALOGUE (tabl. IV)}

\section{- Autres variantes de forme B (fig. 44)}

Il peut paraître étonnant que deux objets découverts presque intacts ne présentent pas un rebord qui corresponde bien aux séries trouvées dans la fosse. Mais la fabrication reste tout à fait comparable et, dans le cas du $n^{\circ} 2$, la pâte caractéristique à inclusions blanches est identique à celle des objets $\mathrm{A} 2 \mathrm{n}^{\circ} 1$, fig. 35 et $\mathrm{n}^{\circ} 7$, fig. 36. La forme générale est classique, galbée, élégante pour le premier, et trapue, lourde, "locale" pour le second. Aussi ne doit-on pas les dissocier de l'ensemble, mais plutôt évoquer une modification du geste des potiers ou une attribution à d'autres séries que l'on ne retrouve ici que de façon ponctuelle, ce qui ne permet guère de leur fixer une référence typologique précise. On retrouve cependant les mêmes variantes de rebords qui caractérisent les ollae, ce qui renforce l'hypothèse d'une production homogène locale.

\section{- Forme B1 (fig. 45)}

Caractères: rebord simple, d'épaisseur comparable à celle de la panse, constitué souvent d'une sorte de bourrelet, sans gorge intcrnc marquée. L'épaulement ou carène peut être situé très haut $\left(\mathrm{n}^{\circ} 1\right.$ à 3$)$, mais le plus souvent il est peu marqué et placé assez bas, ce qui donne une allure générale moins galbée, plus molle $\left(\mathrm{n}^{\circ} 4\right.$ à 7$)$. Les diamètres sont très variables, entre 15,5 et $26 \mathrm{~cm}$.

- Forme B2 (fig. 46)

Caractères : comme pour les formes A2, la section du rebord est simple, de même épaisseur que la panse, et comporte une gorge interne marquée. Les profils les plus courants ( $\mathrm{n}^{\circ} 1$ à 9 ) se trouvent sur des objets à épaulement assez bas ou peu marqué, ou même inexistant $\left(\mathrm{n}^{\circ} 1\right.$ et 5$)$, ce qui leur confère un caractère plutôt "local". Par contre, les fragments $n^{\circ} 10$ et 11 , d'un type assez particulier, montrent une carène bien marquée. L'objet $\mathrm{n}^{\circ} 9$, avec un diamètre de $9 \mathrm{~cm}$ seulement, est de taille exceptionnellement réduite. Les autres diamètres sont compris entre 13 et $20 \mathrm{~cm}$. On retrouve la même proportion que pour les types $\mathrm{A} 2$ au sein des formes $\mathrm{A}$. 

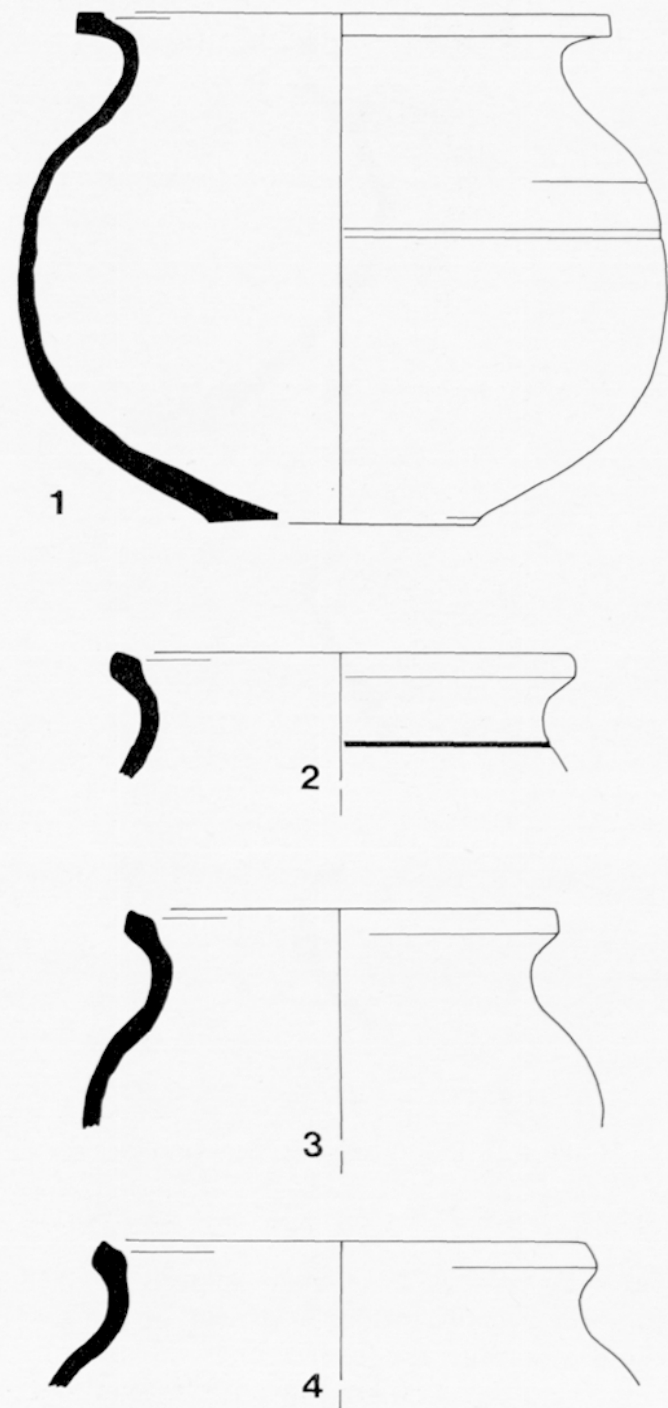

Commune grise. Forme A3

[éch. 1/3]

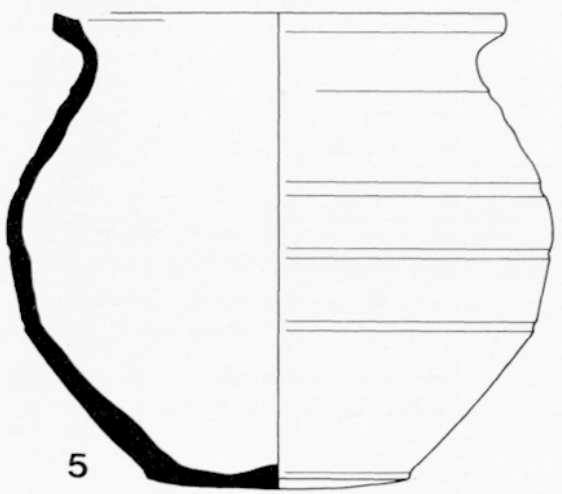

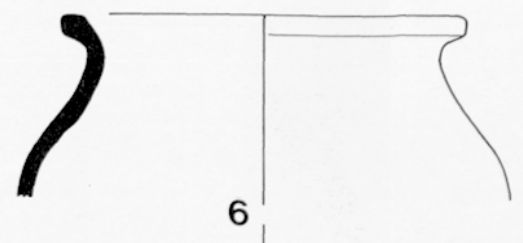
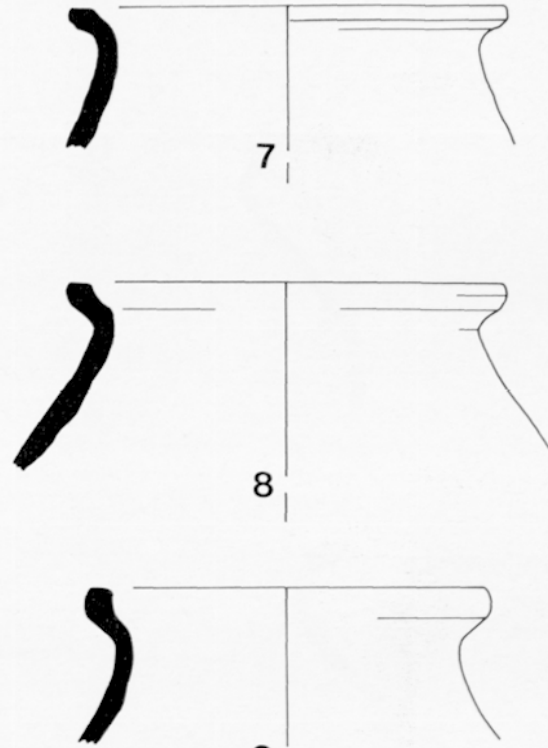

9
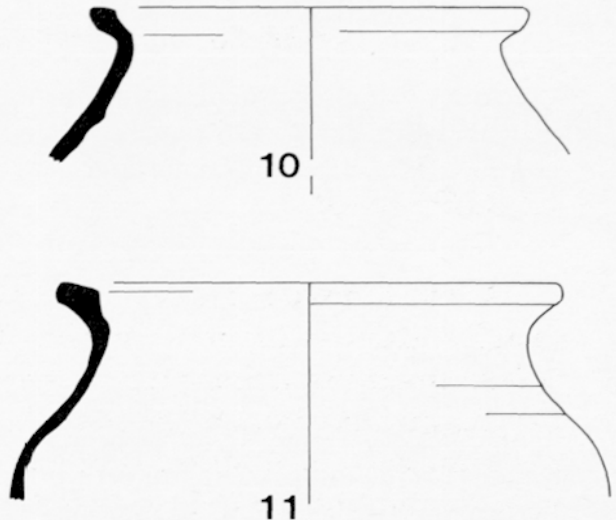

11

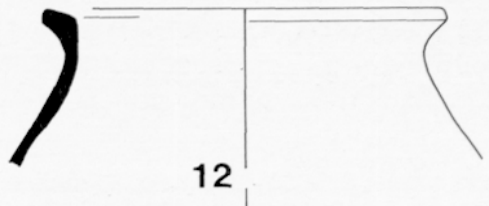

\begin{tabular}{|l|c|c|c|c|c|c|}
\hline Formes & B & B1 & B2 & B3/B3B & B7 & Total \\
\hline NMI & 2 & 16 & 30 & 23 & 4 & $75 e x$ \\
\hline$\%$ & 2,6 & 21,3 & 40 & 30,6 & 5,3 & $100 \%$ \\
\hline
\end{tabular}

Tabl. IV Formes B : répartition par variantes.
- Forme B3 (fig. 47)

Caractères : comme pour les profils $\mathrm{A} 3$, les rebords ont une section à peu près carrée, plus épaisse que la panse, avec un replat supérieur horizontal ou légèrement incliné vers l'intérieur. L'arête extérieure est à peu près verticale ( ${ }^{\circ} 1$ à 6 ). 

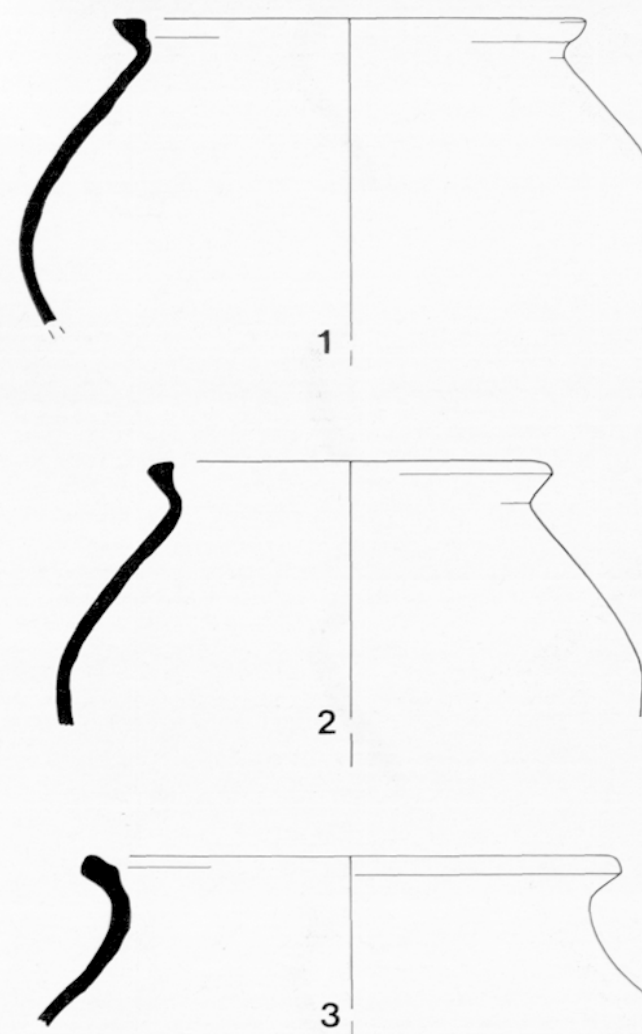

3
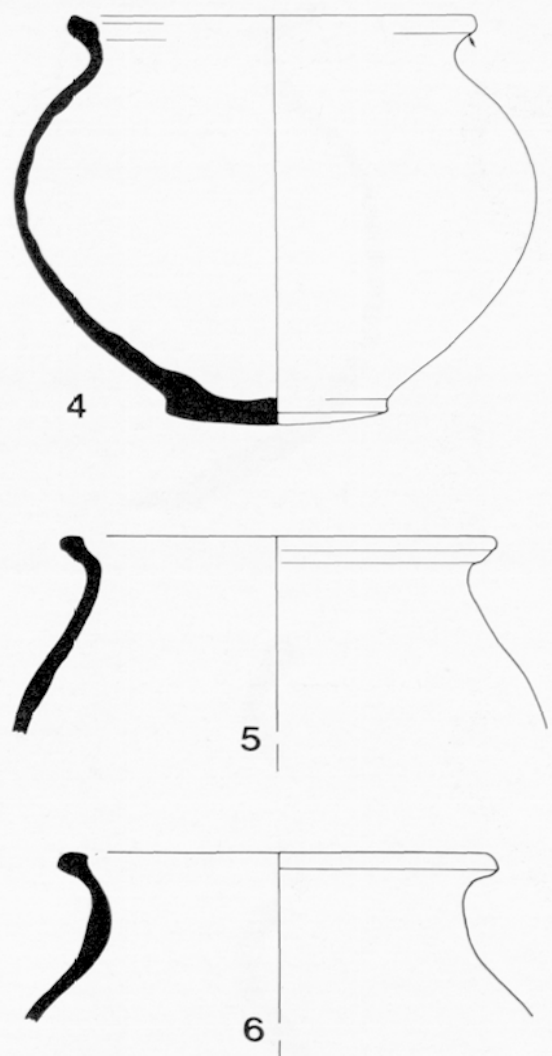

140 Commune grise. Forme $A 3$, variantes. $N^{\circ} 1$ et 2 : forme $A 3 T$, triangulaire, fabrication "locale" ; 3 à 6 : variantes isolées [éch. 1/3]

Quelques variantes proches sont tournées suivant les mêmes principes $\left(n^{\circ} 7\right.$ et 8$)$. On remarque sur l'objet $n^{\circ} 3$, presque complet, la section différente que peut présenter un même rebord.

Les diamètres sont moyens, entre 16 et $21 \mathrm{~cm}$. La plupart des panses ont un épaulement assez marqué, placé haut.

\section{- Forme B3B (fig. 48)}

Caractères : constituant une petite série bien affirmée à Gardanne, cette variante de la forme $\mathrm{B} 3$ a un rebord au profil caractéristique qui présente un petit bourrelet occasionné par l'écrasement de la lèvre vers l'intérieur en fin de tournage. Un exemple isolé de ce type avait été remarqué à Saint-Blaise (Démians d'Archimbaud et al. à paraître, fig. 130, $\mathrm{n}^{\circ} 89$ ). On retrouve sur l'objet $n^{\circ} 1$ la même allure particulièrement lourde due à l'absence d'épaulement que sur le $n^{\circ} 2$ (fig. 44). Les diamètres sont moyens, entre 15 et $20 \mathrm{~cm}$.
Les 23 objets de type $B 3$ et $B 3 B$ représentent près du tiers des formes $\mathrm{B}$, soit encore la même proportion déjà notée au sujet des formes A3.

- Forme B7 (fig. 50)

Cette forme retrouvée en quatre exemplaires vient compléter la typologie. A Saint-Blaise (Démians d'Archimbaud et al. à paraître, fig. 142, $\left.\mathrm{n}^{\circ} 154-156\right)$, les quelques fragments comparables avaient été classés avec les formes rares mal définies. Mais sa répétition nous autorise maintenant à les rattacher aux formes $\mathrm{B}$.

Caractères : les rebords sont de différents types, soit à rapprocher du type B2 ( $n^{\circ} 1$ et 2 ), soit exceptionnels ( $n^{\circ} 3$ et 4 ). Mais les diamètres sont importants, supérieurs à la moyenne (entre 20 et $24 \mathrm{~cm}$ ), et la section de la panse plutôt verticale et rectiligne. On retiendra l'association de ces deux critères pour différencier le type B7, qu'il importera de préciser avec d'autres découvertes.

\begin{tabular}{|l|c|c|c|c|c|c|c|}
\hline Formes & fig./ $\mathbf{n}^{\circ}$ & $\begin{array}{c}\text { diam. bord } \\
\text { (en cm) }\end{array}$ & $\begin{array}{c}\text { diam. max. } \\
(\mathrm{en} \mathrm{cm})\end{array}$ & $\begin{array}{c}\text { diam. fond } \\
\text { (en cm) }\end{array}$ & $\begin{array}{c}\text { hauteur } \\
(\mathrm{en} \mathrm{cm})\end{array}$ & $\begin{array}{c}\text { angle fond } \\
\text { (en degrés) }\end{array}$ & $\begin{array}{c}\text { contenance } \\
\text { (en litres) }\end{array}$ \\
\hline B & $44 / 1$ & 16 & 16,5 & 7 & 9,5 & 30 & 1,1 \\
B & $44 / 2$ & 16,5 & 16 & 8,5 & 7,5 & 30 & 0,9 \\
B1 & $45 / 1$ & 16 & 14 & 7 & 7,5 & 45 & 0,7 \\
B1 & $45 / 3$ & 16,5 & 15,5 & 5,7 & 8,5 & 30 & 0,85 \\
B2 & $46 / 1$ & 17 & 15 & 7 & 8,5 & 40 & 0,85 \\
B3 & $47 / 3$ & 21,5 & 21 & 7 & 11,5 & $35-40$ & 2,35 \\
B3B & $48 / 1$ & 20 & 19 & 9 & 12 & 30 & 2 \\
B3B & $48 / 4$ & 15 & 14 & 5,5 & 6,5 & $30-35$ & 0,6 \\
\hline
\end{tabular}

Tabl. V

Mesures des formes $B$ complètes. 

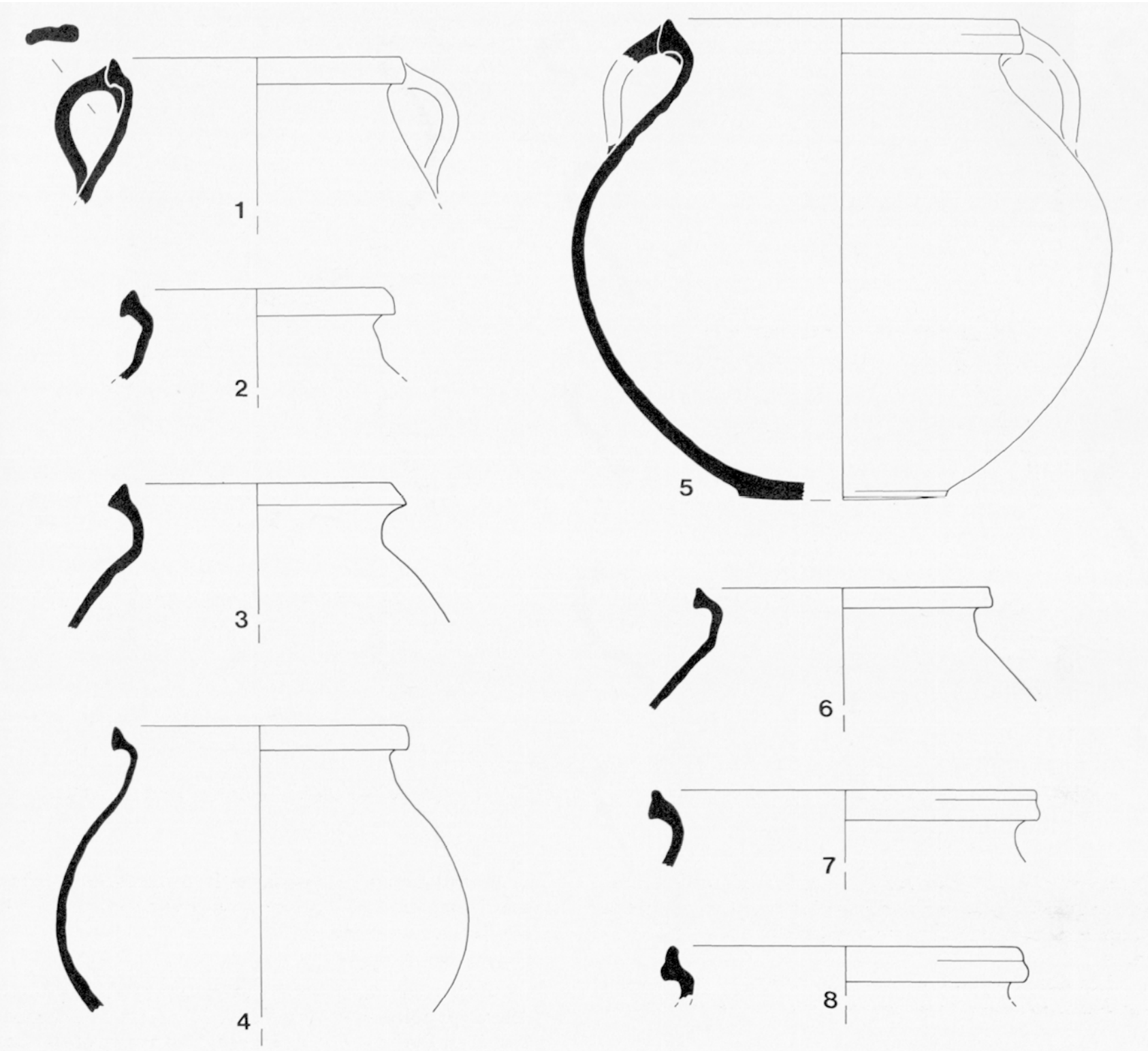

$\square 41$ Commune grise. Formes A4 et A4A [éch. 1/3].

LES DIMENSIONS ET LEURS RAPPORTS

Les formes B ont des dimensions très diverses (tabl. V) : les diamètres sont compris entre 15 et $25 \mathrm{~cm}$ (diamètre moyen : $18 \mathrm{~cm}$ ), et les contenances varient entre moins d'un demi-litre et trois litres. La question de la forme des panses a déjà été évoquée, et en l'absence de séries plus conséquentes il paraît difficile d'en dire davantage. La description des fonds aussi a déjà été abordée ; on peut noter toutefois que l'angle compris entre la base de la panse et l'horizontale, s'il peut varier entre $30^{\circ}$ et $45^{\circ}$, se situe le plus souvent entre $30^{\circ}$ et $35^{\circ}$.

Le rapport entre le diamètre maximum et la hauteur semble assez caractéristique (fig. $43 \mathrm{D}$ ). Les coefficients calculés s'organisent autour d'un axe rectiligne. Les formes "classiques" (bien galbées) plus ou moins basses se situent sur cet axe, ou légèrement au-dessous (rapport compris entre 1,9 et 2,3 ). Par contre, les deux formes lourdes "locales" se retrouvent au-dessus (rapport inférieur à 1,7), l'évolution en fonction de la taille des objets étant parallèle.

Même si les mesures ne portent que sur 8 objets, le graphique paraît déjà significatif, d'autant que la comparaison avec une dizaine d'autres formes régionales complètes (SaintBlaise et Saint-Jean-de-Garguier) confirme ces constatations : les coefficients compris entre 1,9 et 2,2 correspondent à des formes classiques, ceux égaux ou supérieurs à 2,3 à des formes particulièrement larges et basses, notamment d'origine plutôt rhodanienne pour deux d'entre elles, très à l'écart (Démians d'Archimbaud et al. à parâtre, fig. 144).

Curieusement, l'axe de la progression du rapport diamètre du rebord/hauteur forme avec l'horizontale un angle de $35^{\circ}$, qui correspond à l'inclinaison moyenne des bas de panse des formes $\mathrm{B}$. Le même phénomène a été remarqué au sujet des formes $A$, pour lesquelles cet axe (fig. 43B) forme un angle de $45^{\circ}$, soit l'inclinaison moyenne des bas de panse des formes $A$. 
$>$

1
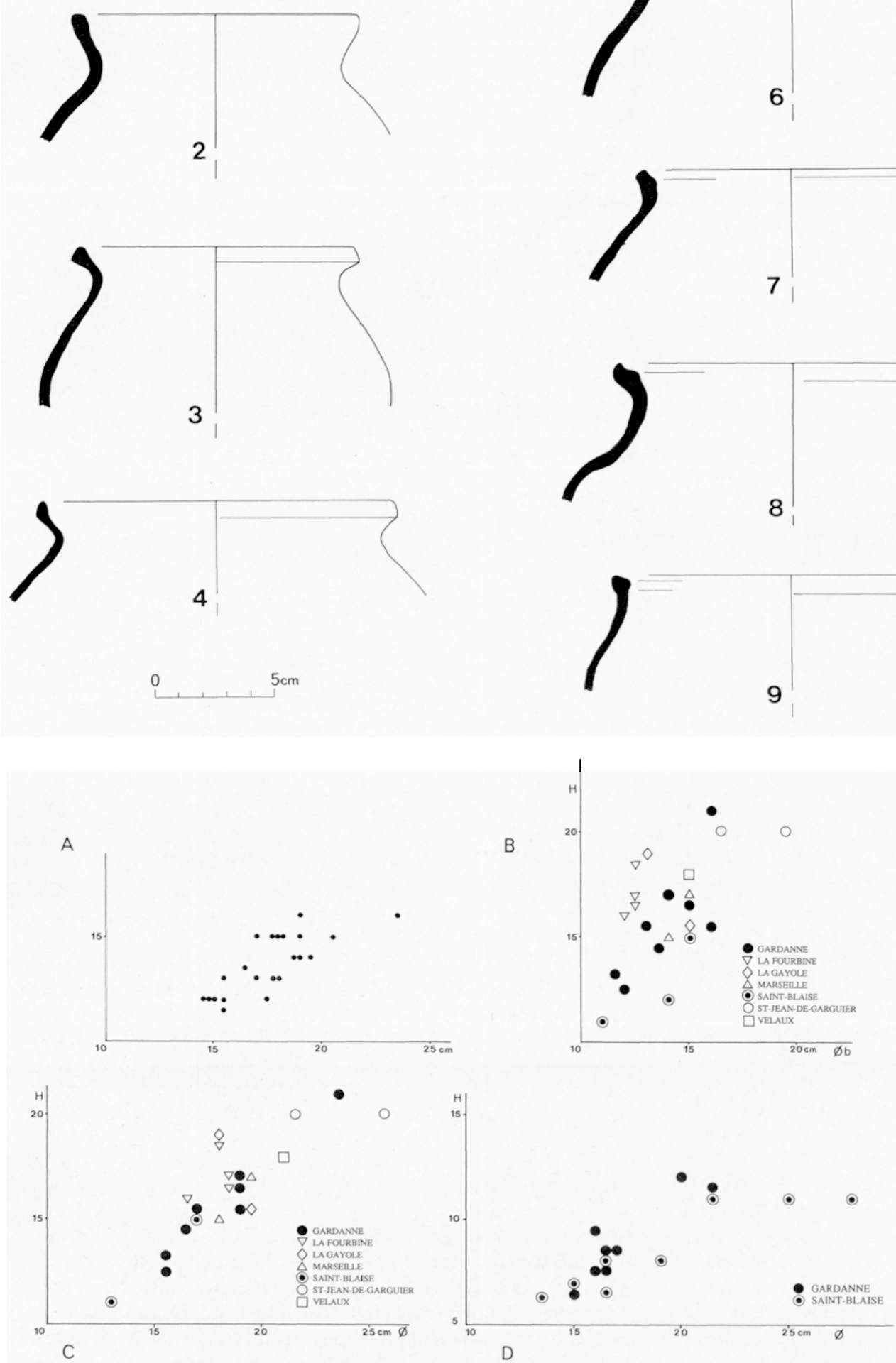

143 Commune grise. A : formes A ; rapport entre le diamètre du bord (ordonnée) et le diamètre maximum de la panse (abscisse). B : formes A; rapport entre le diametre du bord et la hauteur. $C$ : formes $A$; rapport entre le diamètre maximum de la panse et la hauteur. D : formes B.; rapport entre le diametre maximum et la hauteur.

\section{2}

Commune grise. Forme A types divers et isolés [éch. 1/3].

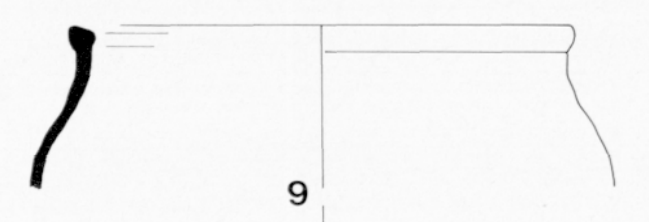

\subsubsection{Les autres formes}

- Forme AB 36 (fig. 51)

Cette forme est caractérisée par la présence d'un bec tubulaire sur un vase à large ouverture et panse globulaire, avec ou sans carène. Bien connue en DS.P. (forme 36 ; Bonifay 1986, 216219), elle n'avait jamais été identifiée en céramique commune grise jusqu'à ce qu'un exemplaire dissocié de tout contexte soit découvert dans les réserves de Glanum, au dépôt archéologique de Saint-Rémy-de-Provence, lors des recherches sur la céramique de Saint-Blaise. Aussi a-t-il paru intéressant de faire figurer ici ce fragment encore inédit $\left(n^{\circ} 1\right)$.

Il est pratiquement certain qu'il s'agit de copies d'objets en céramique fine réalisées par des potiers en céramique commune. Les proportions constatées sur les DS.P. montrent une allure 

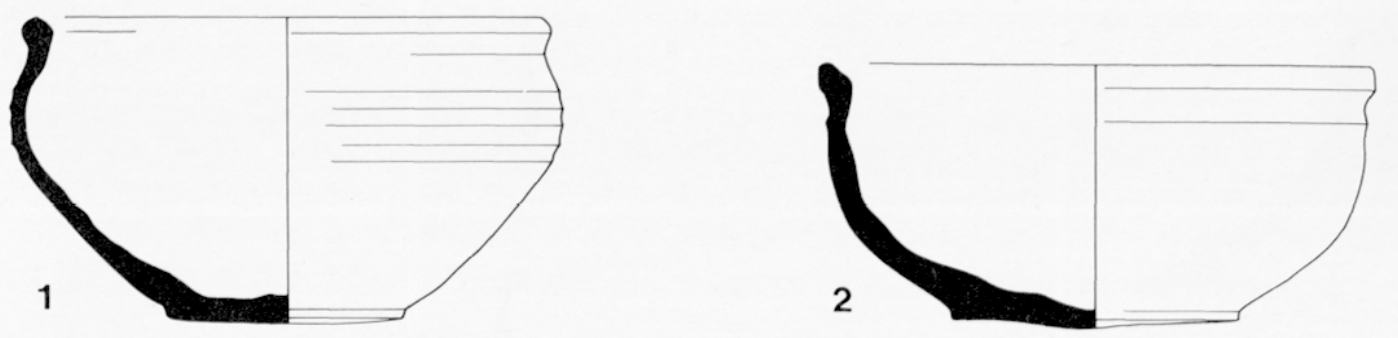

144 Commune grise. Formes B avec rebords de type isolé [éch. 1/3].

45 Commune grise. Forme B1 [éch. 1/3].
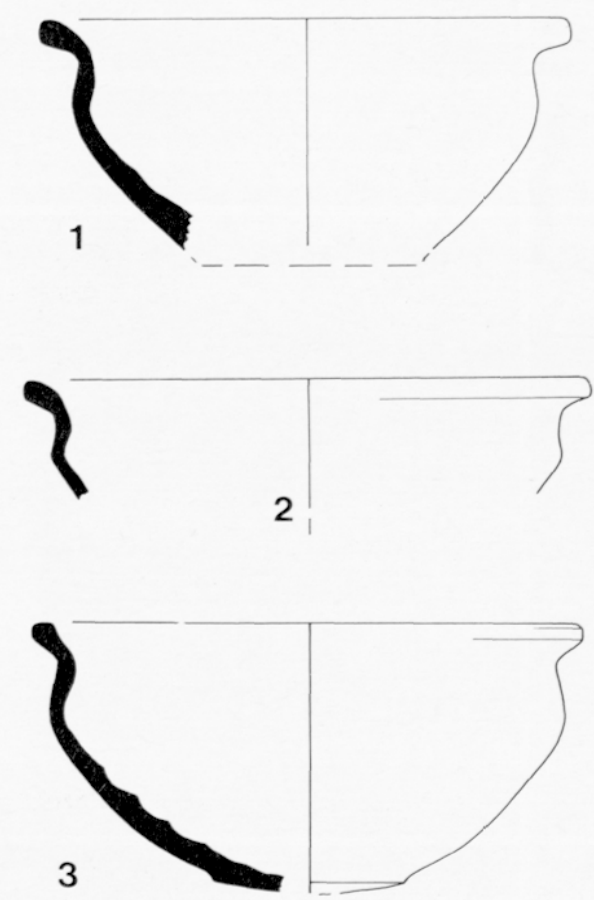

générale des vases à mi-chemin entre les formes $\mathrm{A}$ et $\mathrm{B}$. La hauteur que l'on peut restituer pour l'objet de Gardanne donne un coefficient de 1,37 pour le rapport diamètre du bord/hauteur et 1,58 pour le rapport diamètre maximum/hauteur, ce qui le situe bien nettement entre les deux catégories, et confirme la spécificité de ces poteries.

Selon toute vraisemblance, on doit imaginer une anse verticale à l'opposé du bec tubulaire cylindrique collé à la lèvre dans le cas de Gardanne $\left(n^{\circ} 2\right)$ et nettement dissocié dans celui de Glanum.

Les différentes questions posées par la forme AB 36 seront abordées dans le chapitre 2.6. traitant des interférences entre les DS.P. et les communes, comme pour la forme D.

\section{- Forme D (fig. 52)}

Les mortiers de Gardanne présentent des rebords de formes diverses, très classiques avec la collerette et la lèvre supérieure bien dégagées $\left(\mathrm{n}^{\circ} 1\right.$ à $\left.4,6,7 \ldots\right)$, mais aussi des variantes abâtardies fabriquées avec beaucoup moins de soin, où la section devient presque triangulaire $\left(n^{\circ} 12,13 \ldots\right)$ bien que l'on y trouve encore les mêmes principes, sur des panses plus ou moins inclinées.
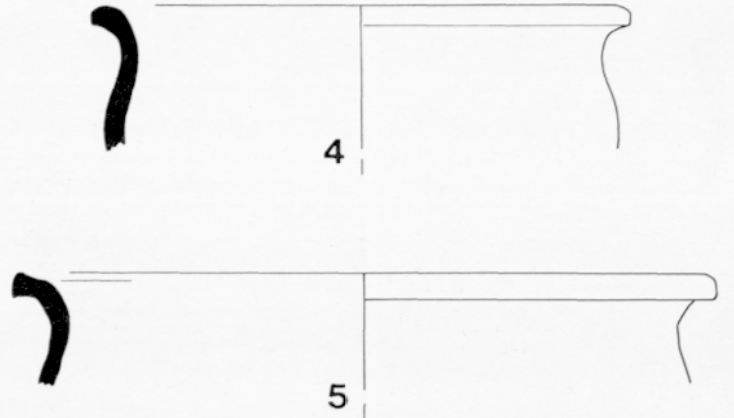

6

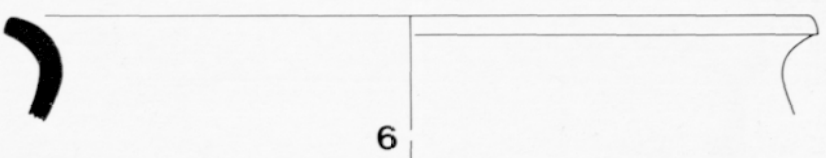

I

7

Les becs verseurs sont de deux types : à bord découpé $\left(n^{\circ} 4\right)$, ou à bec pincé ( $n^{\circ} 2$ et 3$)$.

L'objet $n^{\circ} 14$ est particulier, avec une collerette recourbée vers le bas et une lèvre supérieure presque inexistante, sur une panse très arrondie faisant penser à celle d'une forme $B$. Dans le Vaucluse, deux rebords de ce type ont été identifiés à Apt (Kauffmann 1987, fig. $11, \mathrm{n}^{\circ} 4$ et fig. 13, $\mathrm{n}^{\circ} 52$ ), mais plus d'une centaine de "bols et mortiers" identiques ont été dénombrés dans le four de Bollène-Jonqueirolle (Thiriot 1986, 248, pl. 7). La pâte du tesson de Gardanne semble indiscutablement de la région marseillaise, aussi peut-on évoquer à ce propos la copie d'une forme d'origine rhodanienne?

Outre le mortier $n^{\circ} 4$, seulement trois fonds comportent des inclusions de basalte permettant leur attribution certaine à la forme $\mathrm{D}$, pour un total de 23 rebords différents. Il faut en conclure que la plupart des fonds étaient dépourvus d'inclusions dures, la rugosité de la pâte grise s'avérant suffisante. Le diamètre important du fond des objets $n^{\circ} 4$ et 5 est exceptionnel $(12$ et $16 \mathrm{~cm})$ : il faut imaginer sur la plupart des mortiers un fond "normal", au diamètre inférieur à $10 \mathrm{~cm}$ (voir paragraphe sur les fonds, fig. 57 et tabl. VI). 

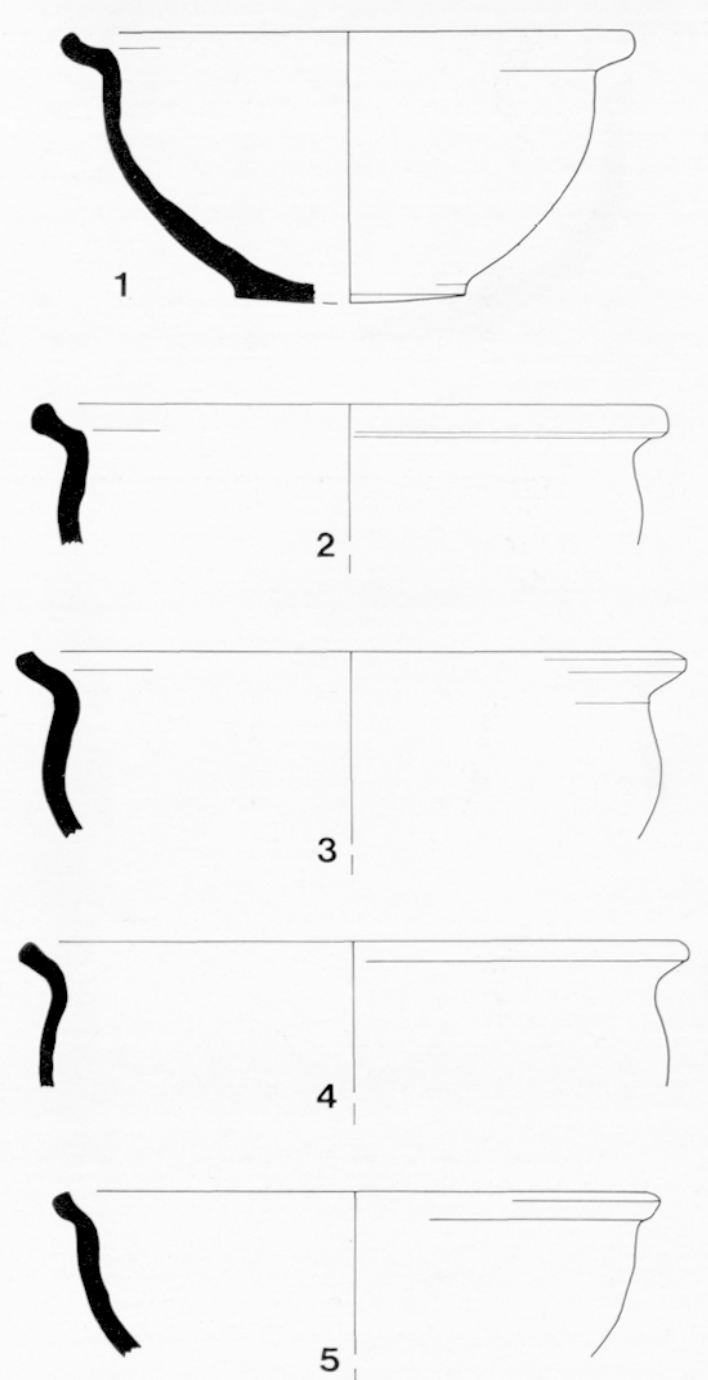
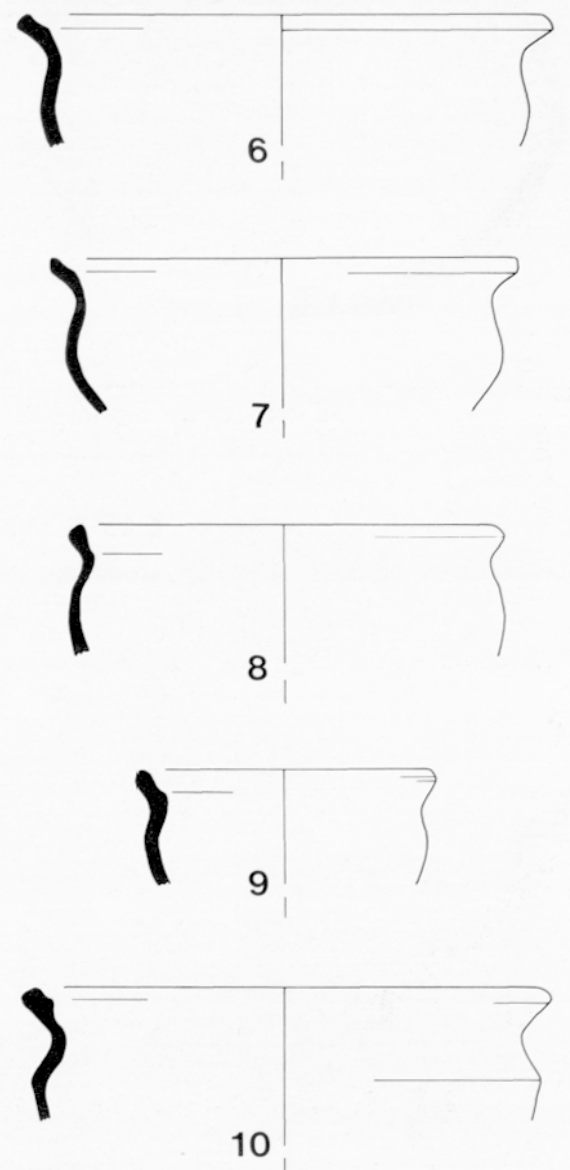

1

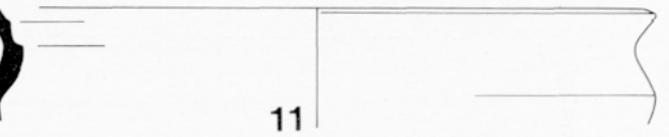

I 46 Commune grise. Forme B2 [éch. 1/3].

Exactement comme à Saint-Blaise, les mortiers représentent $5 \%$ du total des formes répertoriées en commune grise.

- Forme F (fig. 53, n 1 à 3 )

Dans le répertoire des communes grises, l'amphorette est une forme rare, connue seulement par des éléments très fragmentaires, mais qui se trouve mieux définie par les exemplaires connus en DS.P. (forme 63). L'exemplaire le plus important est représenté fig. 62 (chap. 2.6.).

Caractères: rebord de 11 à $12 \mathrm{~cm}$ de diamètre, en poulie. Le col tronconique, bien marqué, est assez large. Il faut imaginer deux anses, fixées sur le ressaut inférieur du rebord.

Les objets $n^{\circ} 1$ et 2 sont très comparables en forme et dimensions à ceux de Marseille (Bonifay 1983, fig. 40, $\mathrm{n}^{\circ} 299-$ 301 et fig. $41, n^{\circ} 315-316$; Cavaillès 1986, fig. $25, n^{\circ} 236$ ), Saint-Blaise (Démians d'Archimbaud et al. à paraître, fig. 138, $\mathrm{n}^{\circ} 137$ et 138 ) et Velaux (Boixadera 1987, fig. 21, $n^{\circ}$ 109). Par contre le $n^{\circ} 3$ est de taille nettement supérieure (diamètre du bord : $14 \mathrm{~cm}$ ), et l'anse particulièrement épaisse recouvre la totalité du rebord.

\section{- Forme K (fig. 53, $\mathrm{n}^{\circ} 4$ à 10)}

Les caractères définissant les cruches précisés dans l'étude du matériel de Saint-Blaise peuvent s'appliquer aux quelques fragments provenant de onze objets : formes à une seule anse, à bec pincé, à col étroit sur une panse globulaire. Les dimensions apparaissent ici assez variables.

L'anse $n^{\circ} 5$ est appliquée sur une panse décorée d'une raie horizontale surmontée d'ondes incisées avec le même outil. C'est le seul véritable décor rencontré sur une céramique commune grise.

Pour mémoire, un tesson proche de l'épaulement d'une forme A ou d'une cruche comporte un graffiti indéchiffrable (fig. 54).

L'anse à poucier $n^{\circ} 10$ est, elle aussi, exceptionnelle.

\section{- Forme L2 (fig. 55)}

Les restes de onze petits bols permettent de définir maintenant la catégorie $\mathrm{L} 2$, avec les objets tout à fait comparables découverts à Marseille (Bonifay 1983, fig. 40, $\mathrm{n}^{\circ} 302$ à 304), Martigues (Pelletier 1984, 183, fig. 1362), et Saint-Blaise (Démians d'Archimbaud et al. à paraître, fig. 140, $\mathrm{n}^{\circ} 145$ ). Caractères : les rebords sont très simples, avec un diamètre de 10 à $12 \mathrm{~cm}$, soit à peu près le double de la hauteur vraisemblale. Les panses sont hémisphériques.

La contenance probable du $n^{\circ} 2$, un quart de litre, est équivalente à celle du bol de Saint-Blaise. Sans doute peut-on évoquer à ce propos une fonction relativement précise pour 

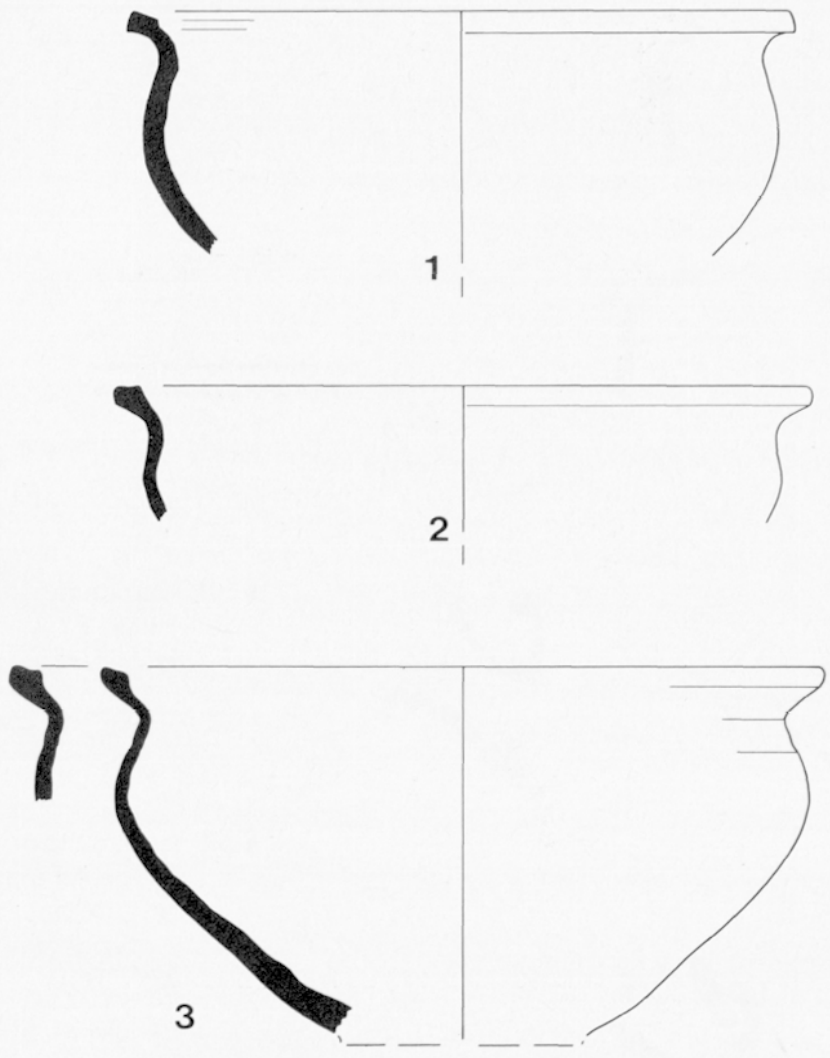

I 47 Commune grise. Forme B3 [éch. 1/3].
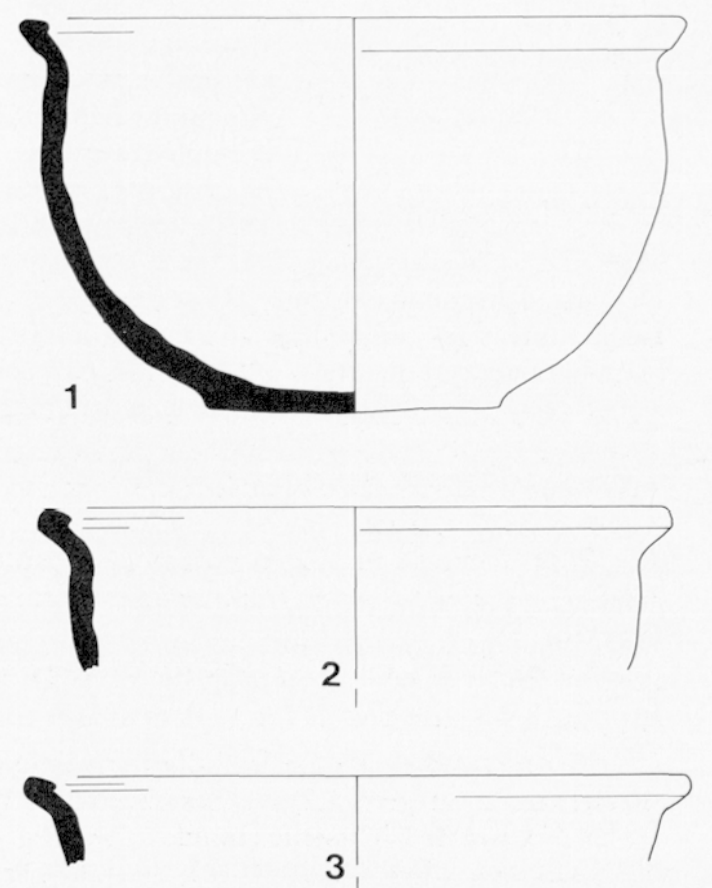

- 48 Commune grise. Forme B3B [éch. 1/3].
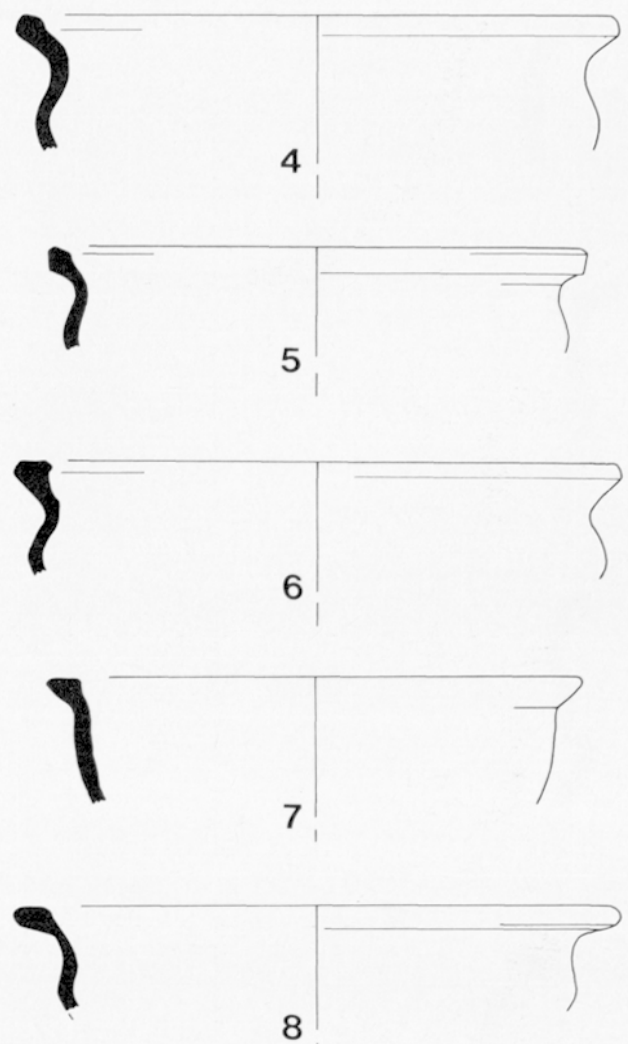

8
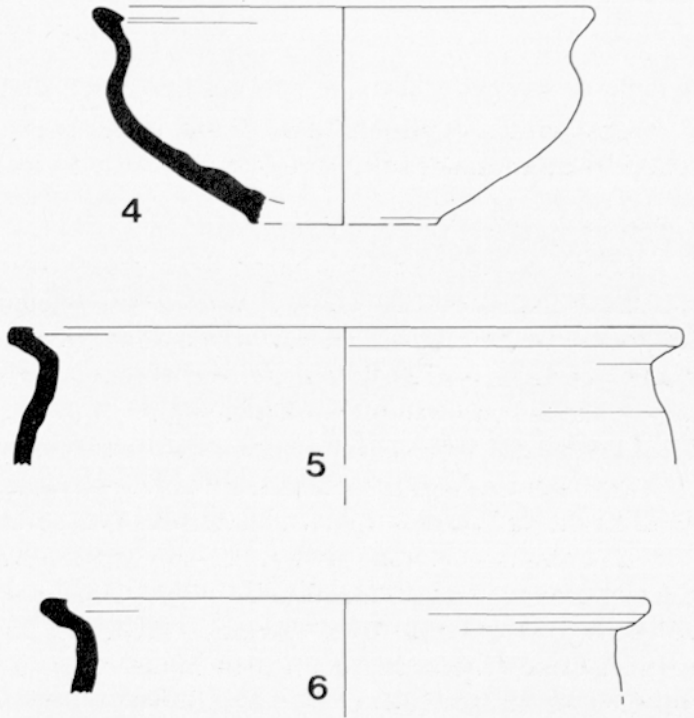

\49 Forme B5. Ce fragment unique n'est pas en céramique commune à pâte grise, mais en pâte commune orangée : il s'agit d'une importation, dont la forme dite "à col vertical épaissi" est fréquente à Arles et dans l'Hérault au Ve s. A Saint-Blaise, cette forme était bien attestée, en pâte orangée et aussi en pâte grise [éch. 1/3]. 

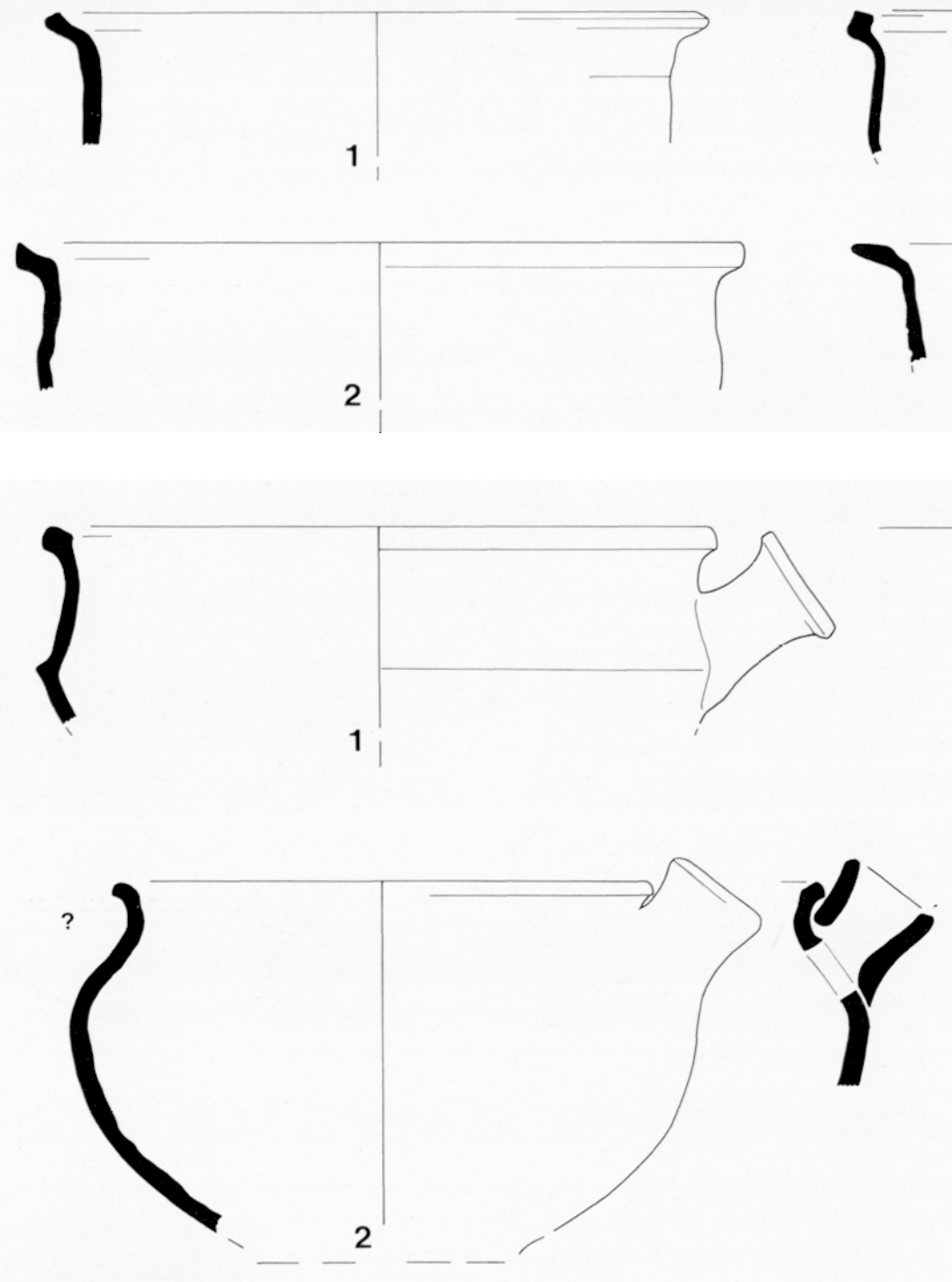

ce type de vaisselle, d'usage peu courant, bien distincte des formes B pour les plus petites, et des bols cylindriques ou tronconiques L1 dont la taille est sensiblement plus importante.

\section{- Forme G (fig. 56, $\mathrm{n}^{\circ} 1$ )}

Bien identifiés à Apt (Kauffmann 1987, fig. 11, $\mathrm{n}^{\circ} 1-3$; fig. 12, $\mathrm{n}^{\circ} 18-21$; fig. $13, \mathrm{n}^{\circ} 41-42$ ) et attestés cn Langucdoc (Raynaud 1990, fig. 123, $n^{\circ} 1-2$ ), les vases de forme $G$ ont une silhouette tronconique avec des parois inclinées de $50^{\circ}$ à $60^{\circ}$. Le fond est très vraisemblablement identique à celui des autres objets. Le rebord est assez large $(20$ à $25 \mathrm{~cm})$.

On ne les retrouve que de façon exceptionnelle en basse Provence : c'est ici le seul exemplaire de la fosse, en pâte locale. Un seul élément est attesté dans les fouilles de Saint-Blaise. Par contre, à Eyguières (prospections J.-P. Pelletier et M. Poguet), ce type apparaît plus fréquent, mais les productions comparables à celles retrouvées à Apt y sont mieux représentées. Ces mêmes remarques s'appliquent à la forme $\mathrm{H}$.

\section{- Forme $\mathrm{H}$ (fig. $56, \mathrm{n}^{\circ} 2$ )}

Cette coupelle de petites dimensions (diam. : $15 \mathrm{~cm}$ ), au profil bien distinct des formes $B$, est aussi un exemplaire unique, de fabrication sans doute locale, peu soignée (variation de la section du rebord).
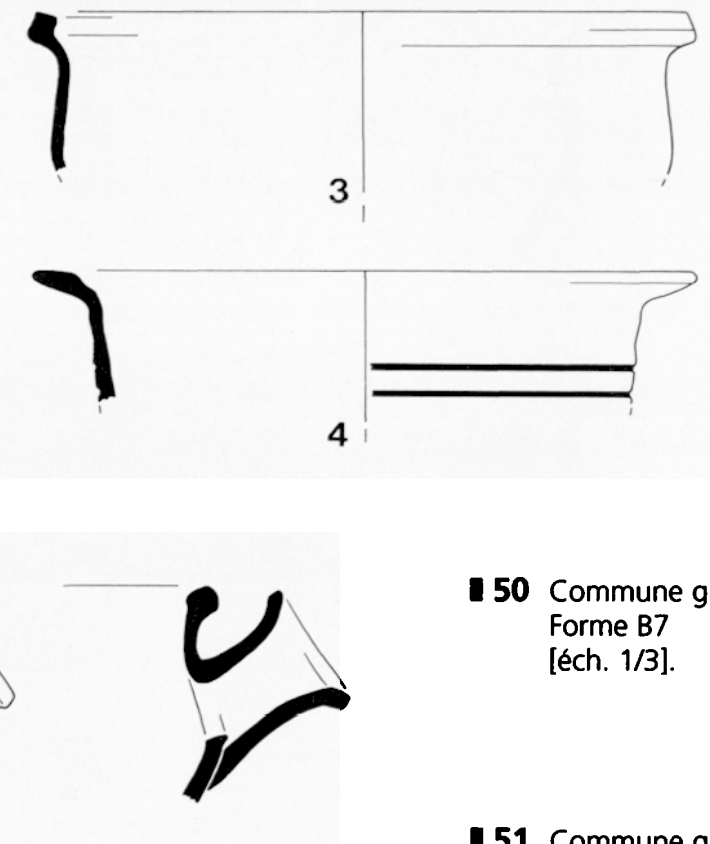

150 Commune grise. Forme B7 [éch. 1/3].

I 51 Commune grise. Forme AB 36. № 1 : Glanum ; 2 : Gardanne [éch. 1/3].
Marseille : Cavalles 1986, 198, fige $27, \mathrm{n}^{\circ} 32 \mathrm{~B}, \mathrm{n} \mathrm{C}^{\circ} 1408$; Démians d'Archimbaud 1980, 279 et fig. 223, n 7), mais plus fréquents sur les céramiques communes importées d'Italie (côte ligure et sud de l'Italie; C.A.T.H.M.A. à paraître, types 7 et 26; Démians d'Archimbaud et al. à paraître, fig. 70).

- Forme rare X (fig. 56, $n^{\circ} 3$ et 4)

Ces deux rebords rappellent des fragments tout aussi exceptionnels retrouvés à Saint-Blaise (Démians d'Archimbaud et al. à paraître, fig. 142, $\mathrm{n}^{\circ} 162-163$ ), Marseille (Bonifay 1983, fig. 39, $n^{\circ} 297$ ) et Saint-Julien-les-Martigues (Pelletier $\left.1984, n^{\circ} 9679\right)$ dans des contextes du VIe $s$. On pourrait les attribuer à des coupelles de forme $\mathrm{B}$, mais avec trop d'incertitudes en l'état actuel des éléments connus.

\section{- Forme L/E (fig. 56, $\mathrm{n}^{\circ} 5$ )}

Cet objet, intermédiaire entre le couvercle et le bol, peut prétendre aux deux modes d'utilisation. La fabrication fruste est celle des couvercles (décollement à la ficelle, déformation de l'intérieur nettement plus accentuée que sur les fonds normaux, épaisseur de la panse...), mais le profil arrondi de la panse et la forme du pied différente des boutons l'apparentent aux petits bols L2 (fig. 55). Sa contenance est tout à fait comparable : 0,24 litre. 

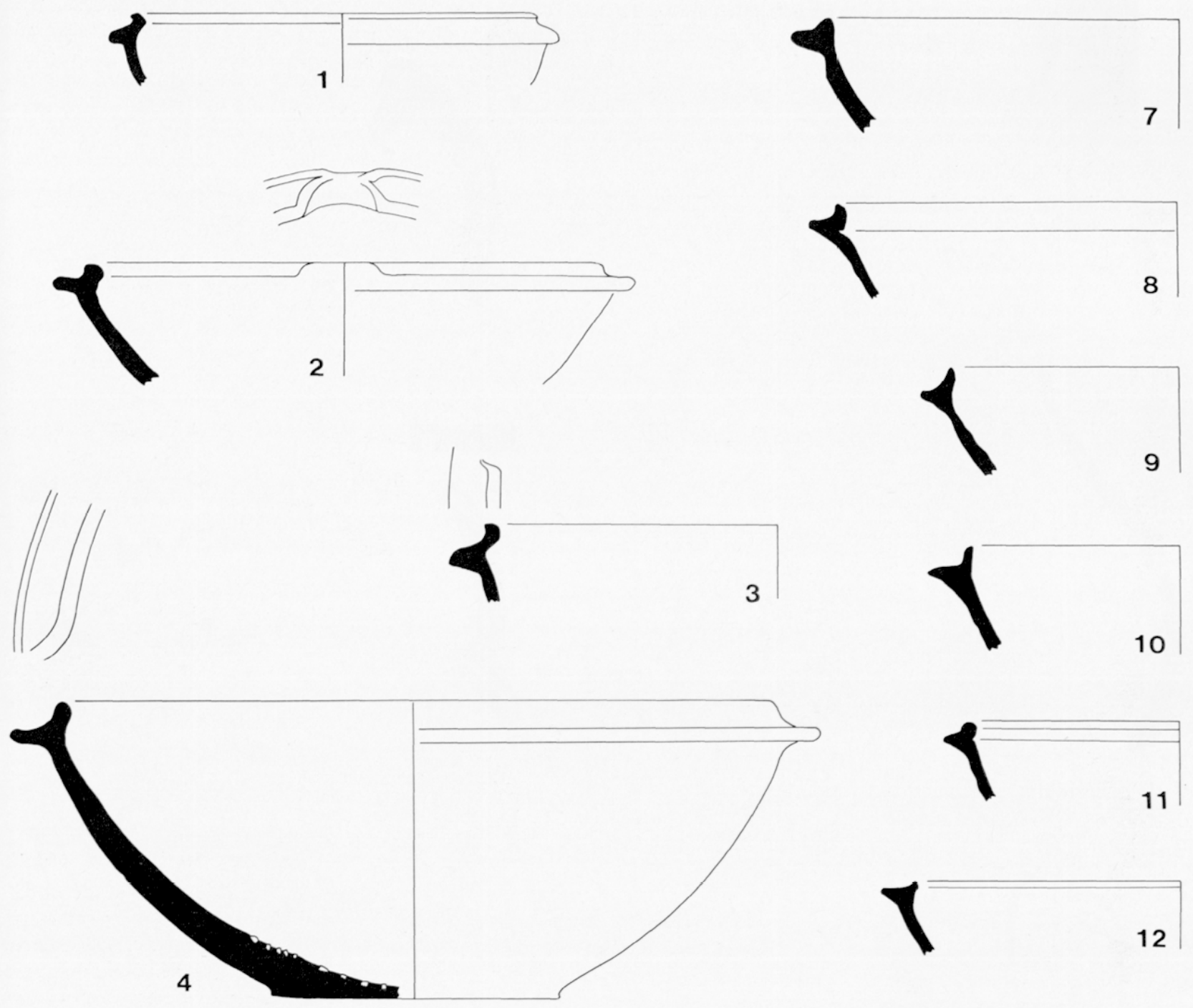

3

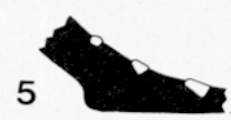

6

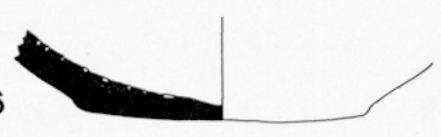

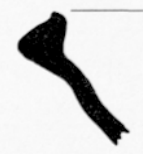
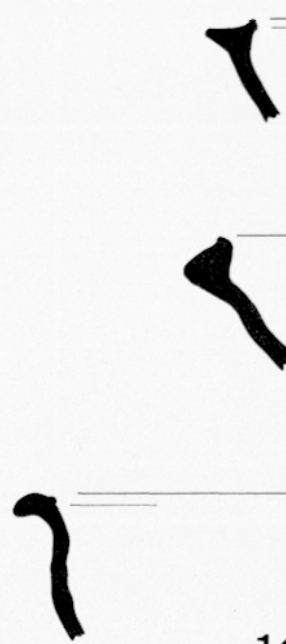

13

52 Commune grise. Forme D [éch. 1/3].

\subsubsection{Les fonds}

\section{FONDS DE TYPE CLASSIQUE}

Leur forme est simple ou à bourrelet peu marqué avec bourrelet ou petit pied (fig. $57, \mathrm{n}^{\circ} 1$ à 21 ).

En additionnant les fonds isolés et les fonds des formes complètes (ceux des formes $\mathrm{D}$ avec inclusions et les petits fragments - comme pour les rebords n'ont pas été comptabilisés), on obtient un total de 275 qui constitue un chiffre minimum (tabl. VI).

Caractères : les fonds sont pratiquement tous rectifiés, le vase étant à l'envers ; cette opération a effacé les traces de décollement (traces que l'on retrouve sur les boutons des couvercles). Variant entre 5 et $10 \mathrm{~cm}$, les diamètres, pour la plupart, sont compris entre 6 et $9 \mathrm{~cm}$, plus des deux tiers entre 7 et $9 \mathrm{~cm}$. La 

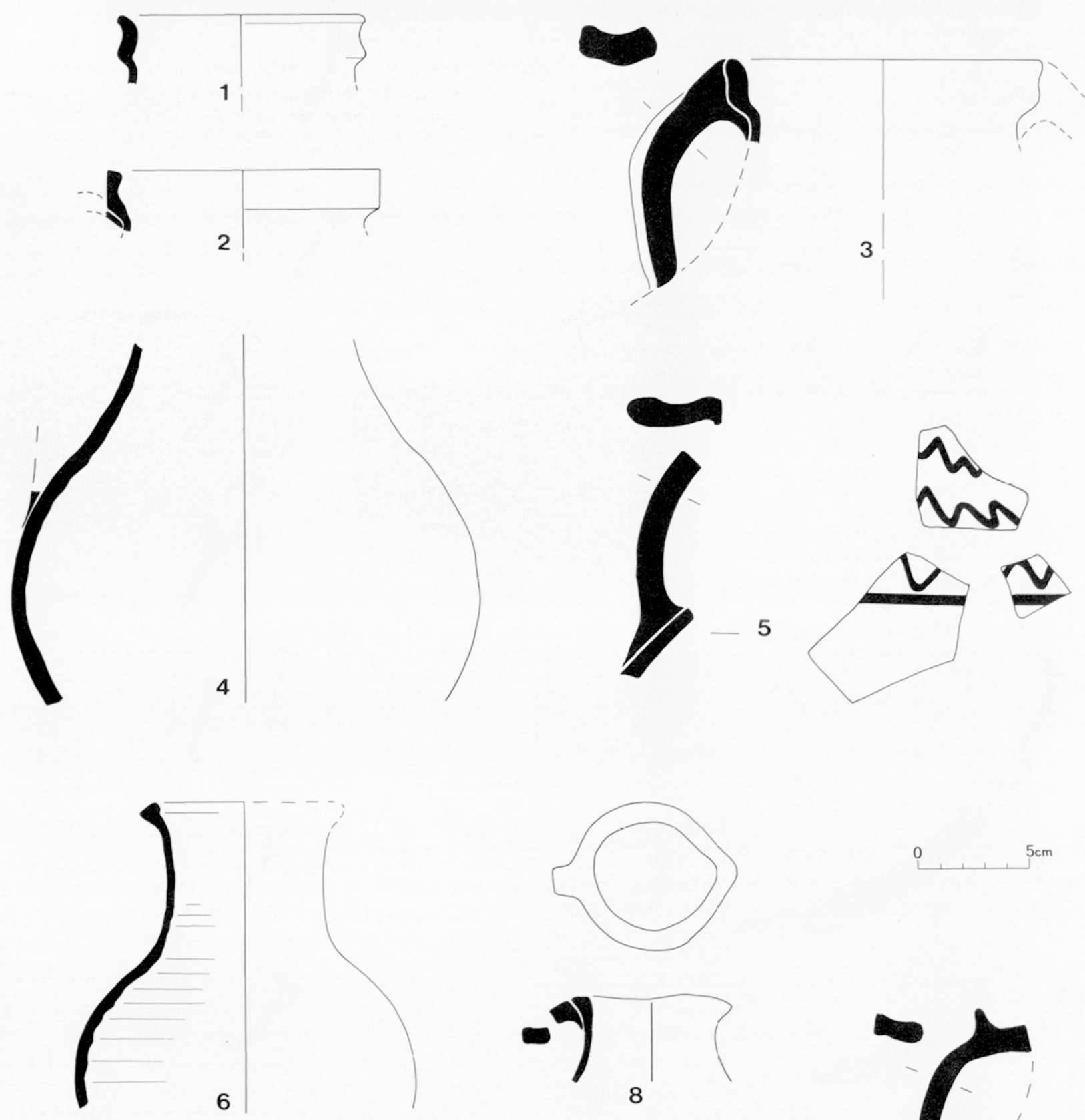

0 $\mathrm{cm}$
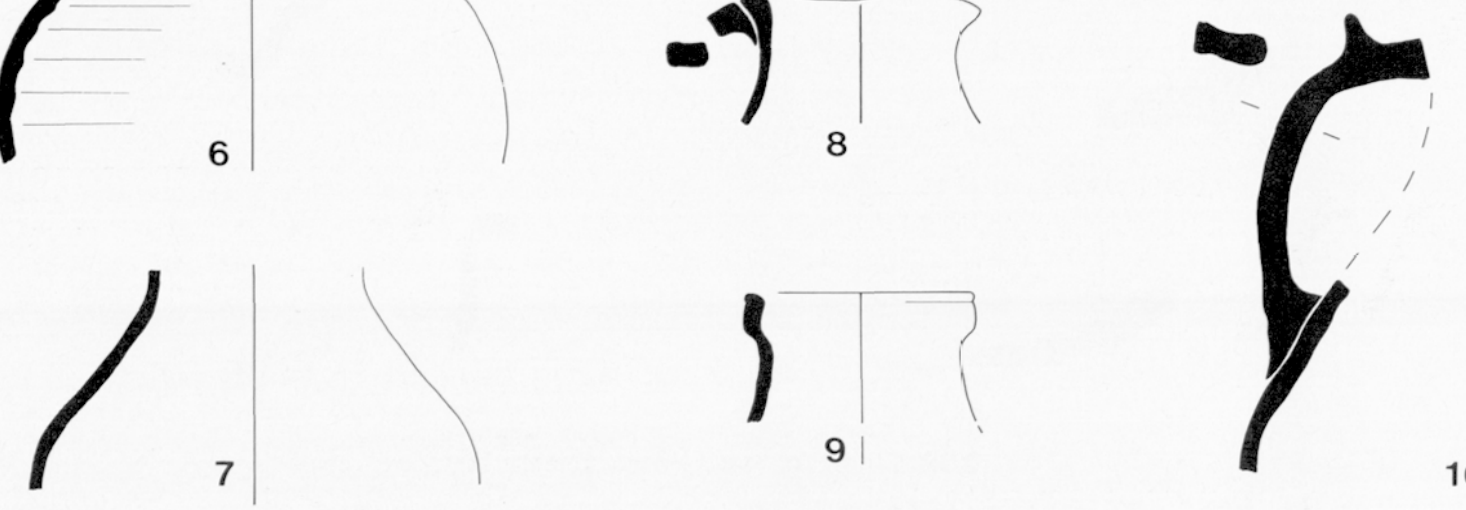

153 Commune grise. $N^{\circ} 1$ à 3 : forme $F$; 4 à 10 : forme $K$ [éch. 1/3].

face inférieure est à peu près plate ou légèrement convexe, très rarement concave. La section est simple $\left(n^{\circ} 1,6,12,14 \ldots\right)$ ou présente un petit pied ou un bourrelet $\left(n^{\circ} 2,5,7,9 \ldots\right)$.
L'angle compris entre la base de la panse et l'horizontale peut varier en fonction de la nature des objets. A partir des quelques mesures effectuées sur les formes complètes, on 


\begin{tabular}{|l|c|c|c|}
\hline $\begin{array}{l}\text { Diamètre } \\
(\mathrm{en} \mathrm{cm})\end{array}$ & $\begin{array}{c}\text { Fonds 1 } \\
\text { (nb ex.) }\end{array}$ & $\begin{array}{c}\text { Fonds 2 } \\
\text { (nb ex.) }\end{array}$ & $\begin{array}{c}\text { Fréq. total } \\
\text { (en \%) }\end{array}$ \\
\hline 5 à 6 & 15 & 4 & 6,9 \\
6 à 7 & 32 & 25 & 20,7 \\
7 à 8 & 41 & 48 & 32,3 \\
8 à 9 & 6 & 94 & 36,3 \\
9 à 10 & 7 & 3 & 3,6 \\
\hline
\end{tabular}

Tabl. VI Fonds classiques; répartition par diamètre et par forme (fonds 1 : forme simple ou à bourrelet peu marqué : fonds 2 : forme avec bourrelet ou petit pied).

154 Commune grise. Fragments de panse à décor ondé (voir fig. 53. $n^{\circ} 5$ ) et fragment de panse avec graffiti [éch. 1/1].

155 Commune grise. Forme L2 [éch. 1/3].

56 Commune grise. $N^{\circ} 1$ : forme $\mathrm{G} ; 2$ : forme $\mathrm{H} ; 3$ et 4 : forme rare ; 5 : forme L/E ; 6 et 7 : mamelons de préhension sur formes $O$ [éch. 1/3].

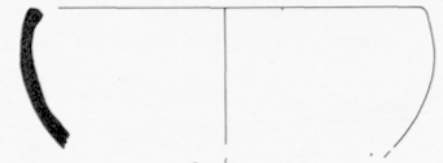

1
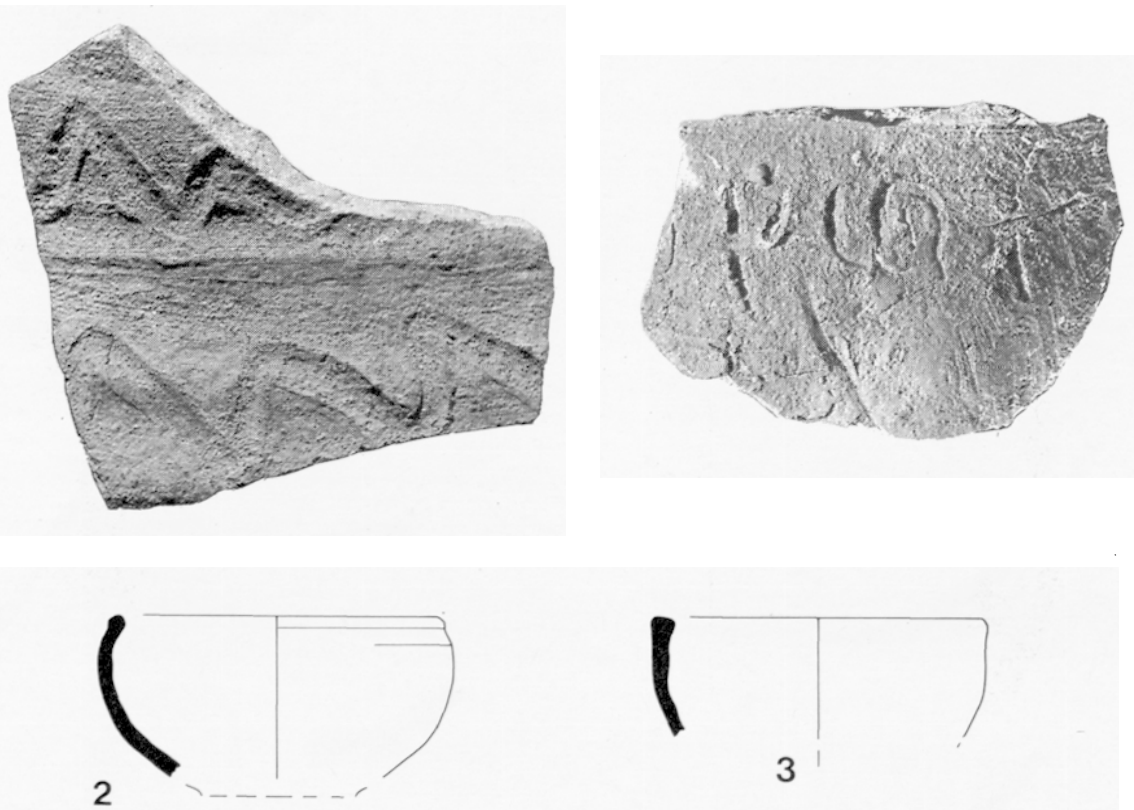

3

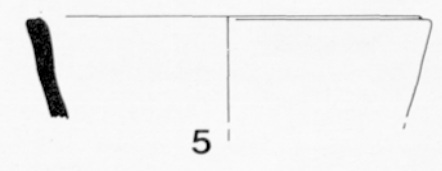

0

4

5
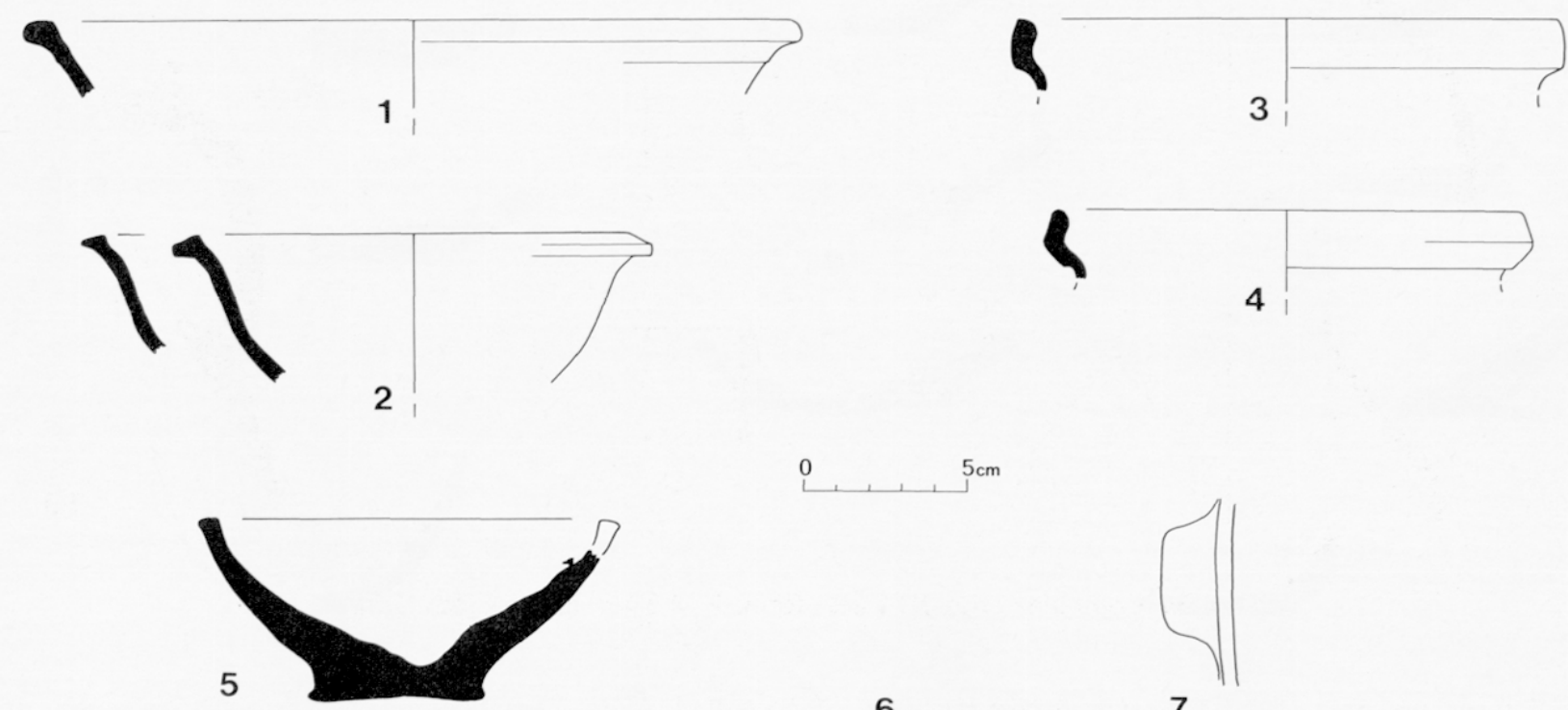

0 $5 \mathrm{~cm}$
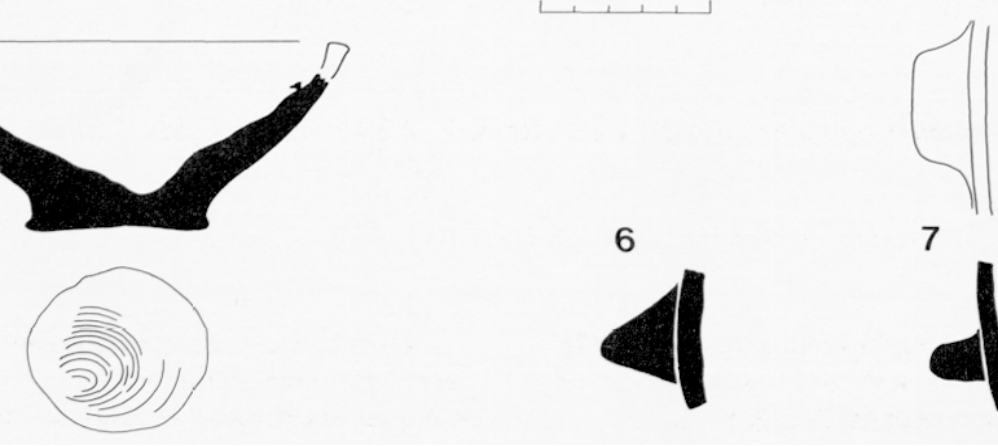

7

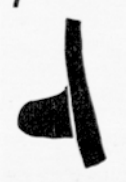



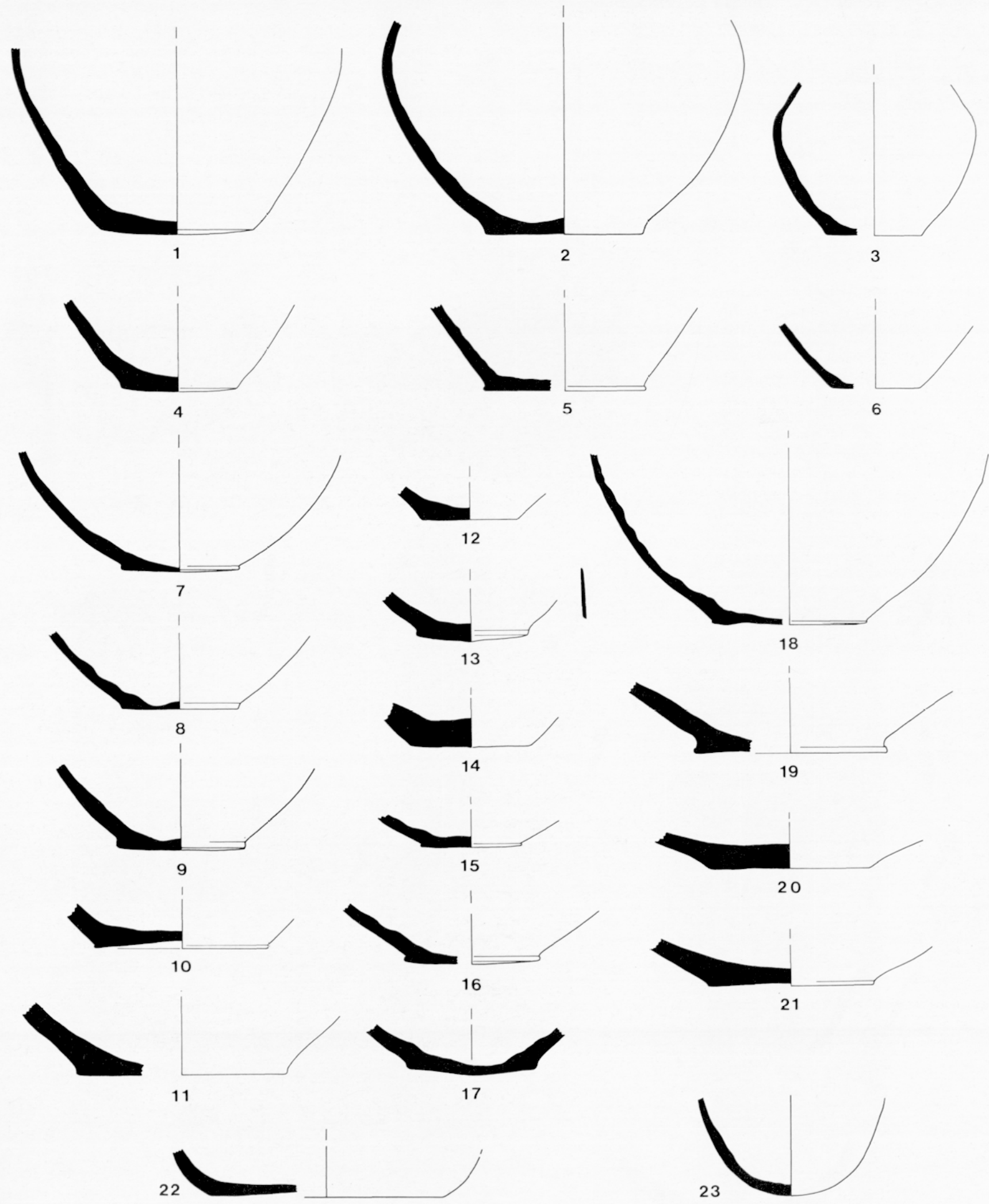

$\square 57$ Commune grise. $N^{\circ} 1$ à 21 : fonds de type classique, formes A, B et autres ; 22 et 23 : fonds de type exceptionnel [éch. 1/3].

peut considérer qu'il se situe en moyenne autour de $40^{\circ}$ pour les formes A (variation entre $30^{\circ}$ et $55^{\circ}$ ) et un peu moins de $35^{\circ}$ pour les formes $B$ (variation entre $30^{\circ}$ et $45^{\circ}$ ).
Il est difficile d'attribuer avec certitude un élément à une forme précise. Statistiquement, la plupart proviennent de formes $\mathrm{A}$ et $\mathrm{B}$, mais la fabrication sommaire, les variations 
d'épaisseur, le fait que les types soient peu différenciés doivent inciter à la prudence. Par exemple, les fonds de mortiers $D$ ne comportant pas d'inclusions ne peuvent être distingués, de même les fonds de cruches, et des autres formes...

\section{FONDS DE TYPE EXCEPTIONNEL}

Le $\mathrm{n}^{\circ} 23$ (fig. 57), pièce unique, pourrait-il être attribué à une sorte de petite amphore ? Le $n^{\circ} 22$ ne correspond, lui non plus, à aucune des formes connues en commune grise.

\subsubsection{Forme $E$ : les couvercles}

On peut estimer leur nombre minimum à 80 objets répartis en quatre types (fig. 58):

- Forme E1 ( ${ }^{\circ} 2$ et 3$)$ : rebord de forme simple (16 exemplaires, soit $20 \%$ ).

- Forme E2 ( ${ }^{\circ} 4$ à 6) : rebord en bourrelet, plat ou arrondi, formant une gorge interne plus ou moins marquée (14 ex., soit 17,5\%).

- Forme E3 ( ${ }^{\circ} 7$ à 10) : rebord assez large, posant plus ou moins à plat (14 ex., soit 17,5\%).

- Forme E4 ( ${ }^{\circ} 11$ à 14) : rebord anguleux, posant sur la pointe ( 36 ex., soit $45 \%$ ).

- Caractères : les couvercles sont de forme tronconique, la panse à peu près rectiligne formant un angle de $30^{\circ}$ à $40^{\circ}$ par rapport à l'horizontale. Ils comportent un bouton de préhension décollé à la ficelle. Le mode de fabrication "à la motte", très rapide, entraîne de fréquentes déformations, défauts de centrage $\left(n^{\circ} 7,21 \ldots\right)$, et cavités sur le bouton $\left(n^{\circ} 18\right.$ à 20$)$.

Pour 27 boutons dénombrés, cinq sont percés afin de laisser un passage à la vapeur $\left(\mathrm{n}^{\circ} 21\right.$ à 23). Le diamètre des boutons, variant entre 3 et $6 \mathrm{~cm}$, est le plus souvent voisin de $4 \mathrm{~cm}$. Le diamètre maximum, variant entre 13 et $18 \mathrm{~cm}$, se situe en moyenne entre 14 et $15 \mathrm{~cm}$. La hauteur est de 6 à $7 \mathrm{~cm}$ environ.

Les couvercles doivent avoir été utilisés essentiellement sur des formes $\mathrm{A}$, et les dimensions des deux types d'objets correspondent bien. Mais il ne semble pas que la gorge interne des rebords A2 ait une fonction en rapport avec la pose des couvercles : en effet, les diamètres ne permettent pas une adaptation systématique dans cette gorge.

L'objet $\mathrm{n}^{\circ} 1$ a un profil bien différent des séries couramment utilisées, et il semble qu'il ait pu aussi servir de bol.
Il comporte une longue lèvre verticale et une ébauche de collerette horizontale. On peut le rapprocher d'un couvercle - moins haut - découvert à Saint-Blaise (Démians d'Archimbaud et al. à paraître, fig. 137, $\mathrm{n}^{\circ} 136$ ), et évoquer alors son adaptation sur une cruche ou amphorette de forme $\mathrm{F}$, les diamètres respectifs correspondant. Les bouchons adaptables sur les cruches $\mathrm{K}$ comportent également une collerette et une lèvre inférieure à peu près verticale, mais leur diamètre est beaucoup plus réduit (Boixadéra 1987, 104, fig. 20, $n^{\circ} 72$; Raynaud 1990, fig. 120, $\left.n^{\circ} 15\right)$. Le rapprochement s'impose avec les formes DS.P. 54 : nous reprenons le même indice pour caractériser cette forme E 54.

\subsubsection{Comptage et répartition des différentes formes}

L'observation des comptages des différentes formes de la fosse de Gardanne entraîne certaines remarques qui précisent l'utilisation de la céramique commune grise à cette époque. La comparaison avec Saint-Blaise, seul site pour lequel on dispose de chiffres portant sur le même matériel, apporte des éléments complémentaires.

\section{$\square$ Les formes A}

Le répertoire de Gardanne est essentiellement constitué des types $\mathrm{A} 1, \mathrm{~A} 2$ et $\mathrm{A} 3$ : avec $85 \%$ du total, ces trois formes caractéristiques de la région marseillaise dominent largement. A Saint-Blaise, où les faciès sont plus différenciés avec les apports de la vallée du Rhône, elles ne représentent plus que le tiers du total $(32,7 \%)$.

\section{$\square$ Les formes B}

En ce qui concerne les formes $B$, le phénomène est encore plus marqué. La catégorie " $B$ autres" comprend les quatre objets B7 (dont deux proches de B2) mais aussi deux objets considérés comme étant de fabrication "locale" malgré leurs profils sensiblement distincts (fig. 44). On peut donc penser que les types régionaux, avec 71 ou 73 pièces, constituent la presque totalité du répertoire, alors qu'à Saint-Blaise les formes $\mathrm{B}$ autres que $\mathrm{B} 1, \mathrm{~B} 2$ et $\mathrm{B} 3$ c'est-à-dire principalement B5 et B4, formes plutôt

Tabl. VII Répartition comparative des formes A, avec pourcentages par rapport au total des formes $A$.

\begin{tabular}{|c|c|c|c|c|c|c|c|}
\hline Formes & A1 & A2 & A2A & A3 & A4 & A autres & Total \\
\hline GARDANNE & 24 & 88 & 10 & 64 & 15 & 18 & $219 \mathrm{ex}$. \\
$\%$ & 11 & 40,2 & 4,5 & 29,2 & 6,8 & 8,2 & \\
\hline SAINT-BLAISE & 15 & 34 & 6 & 13 & 75 & 65 & $208 \mathrm{ex}$. \\
$\%$ & 7,2 & 16,3 & 2,9 & 6,2 & 36 & 31,2 & \\
\hline
\end{tabular}

Tabl. VIII Répartition comparative des formes B, avec pourcentages par rapport au total des formes B.

\begin{tabular}{|c|c|c|c|c|c|}
\hline Formes & B1 & B2 & B3 & B autres & Total \\
\hline GARDANNE & 16 & 30 & 23 & 6 & 75 ex. \\
$\%$ & 21,3 & 40 & 30,6 & 8 & \\
\hline SAINT-BLAISE & 19 & 14 & 19 & 48 & $100 \mathrm{ex}$. \\
$\%$ & 19 & 14 & 19 & 48 & \\
\hline
\end{tabular}



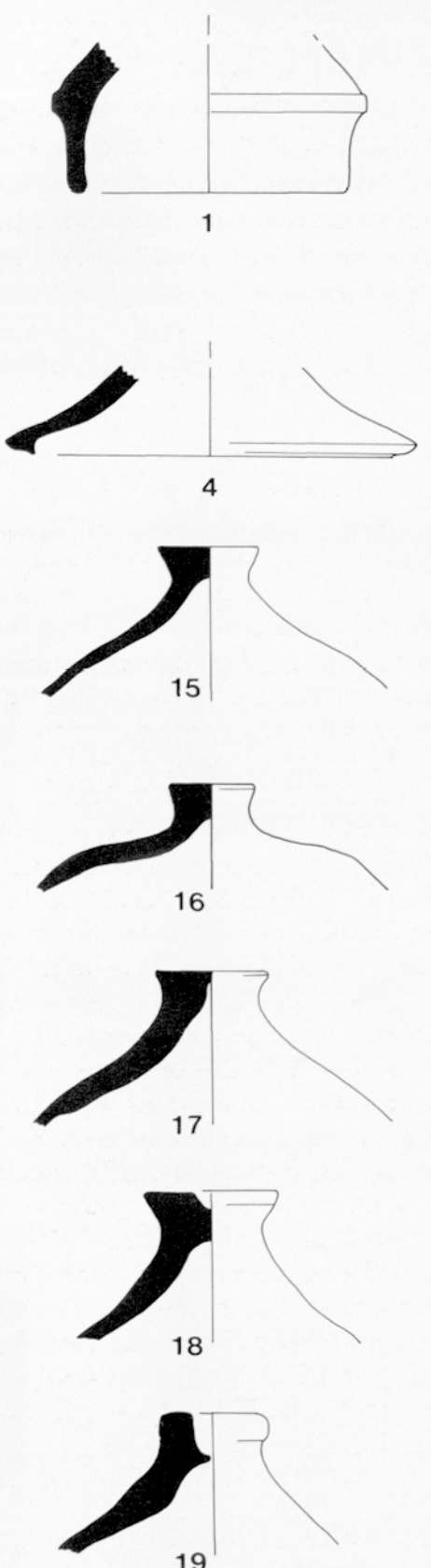

19

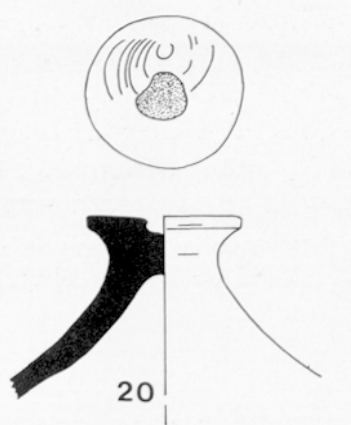

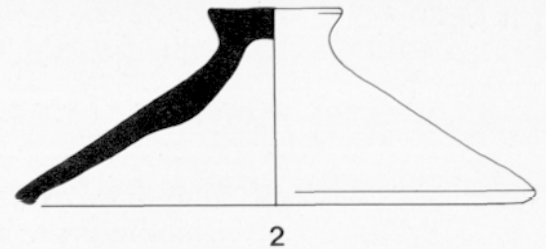
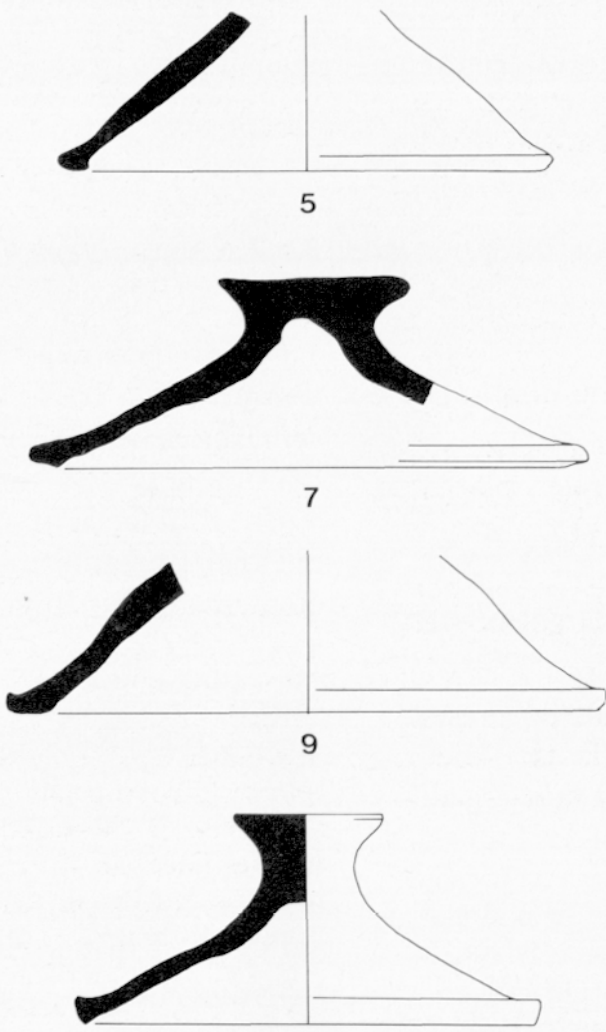

11

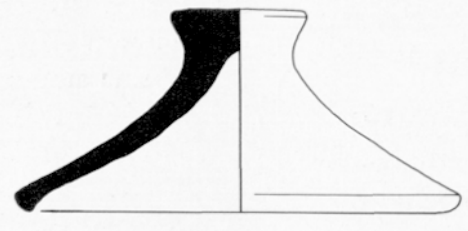

13
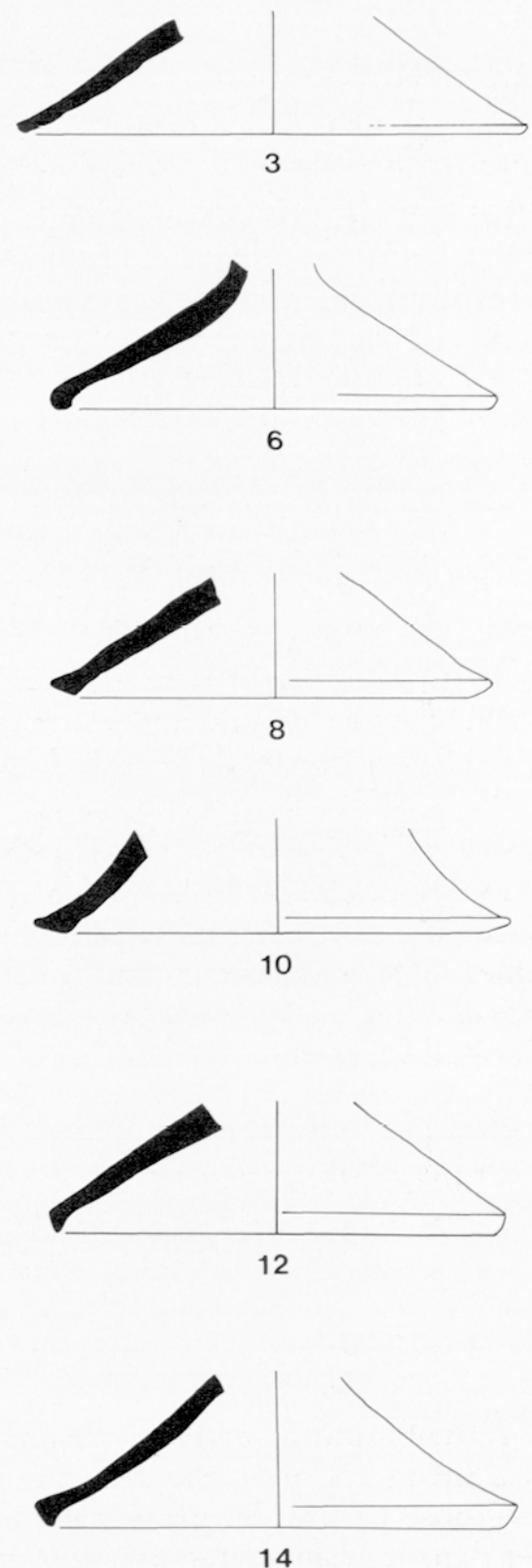

14
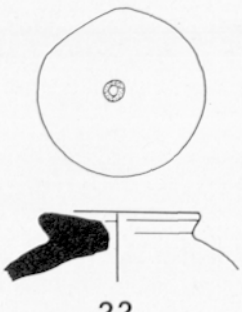

22

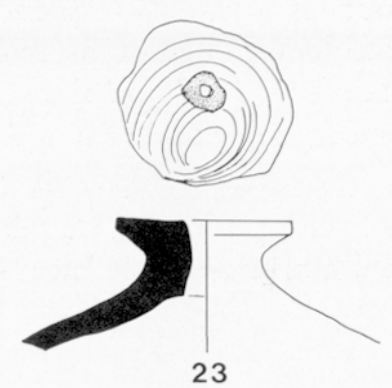

23

21

158 Commune grise. Couvercles. $N^{\circ} 1:$ forme $E 54 ; 2$ et $3:$ forme $E 1 ; 4$ à $6:$ forme $E 2 ; 7$ à $10:$ forme $E 3 ; 11$ à $14:$ forme $E 4$; 20 à 23 : boutons de préhension [éch. 1/3]. 
rhodaniennes - représentent près de la moitié des objets.

\section{$\square$ Ensemble des formes}

Les comparaisons des proportions des différentes formes montrent des analogies assez frappantes entre les deux sites, qui ne sont peut-être que coüncidences et demanderont à être reconsidérées lors d'études ultérieures.

Les formes A représentent la moitié des vases (51,9\% à Saint-Blaise et $50,9 \%$ à Gardanne), les formes $D: 5 \%$ et $5,3 \%$, les formes $F$ et $K: 4 \%$ et $3,2 \%$.

Sensible sans être importante pour les formes B (respectivement $24,9 \%$ et $17,4 \%$ ), la différence est plus affirmée pour les couvercles, trois fois plus nombreux à Gardanne qu'à Saint-Blaise (18,8 \% et 6,2 \%).

Le total des trois formes $A, B$ et $E$ reste très proche dans les deux cas: il est respectivement de $83 \%$ à Saint-Blaise et de $87 \%$ à Gardanne.

On peut dire aussi que les couvercles constituent à peu près le sixième de l'ensemble de la vaisselle commune grise; ils sont aussi nombreux que les formes $B$, et on en compte presque un pour trois formes A. Mais sans doute faut-il voir là des aspects particuliers, dûs à la nature du gisement et de l'habitat dont il dépendait.

\subsection{LES RAPPORTS ENTRE PRODUCTIONS CERAMIQUES}

\subsubsection{Les interférences}

L'attribution de certains tessons à la catégorie des DS.P. plutôt qu'à celle des communes, ou viceversa, pose parfois des problèmes. On retrouve en effet dans les deux catégories des caractéristiques technologiques identiques à la fois pour les pâtes et pour les formes. Ce phénomène avait déjà été pressenti lors de l'étude du matériel de Marseille-Bourse (Bonifay 1983, 334) et mieux observé avec les apports de celui de Saint-Blaise.

Le premier critère de distinction visuelle concerne la composition de la pâte : la présence ou l'absence d'inclusions plus ou moins grossières, la surface plus ou moins douce au toucher peuvent influer sur le choix dans un sens ou dans l'autre. Au stade des comptages, les tessons douteux ont été regroupés avec les DS.P., et les différenciations ne seront jamais assez précises pour en faire une catégorie particulière. Ensuite intervient le critère des formes, et le choix peut être contradictoire car des objets de profil analogue se retrouvent dans les deux répertoires. Principalement, les mortiers, forme 29 (fig. 24) ou D (fig. 52), et les "amphorettes", forme 63 (fig. 25) ou F (fig. 53).

Parmi les mortiers, il n'existe cependant aucun exemplaire équivoque. Par contre, si l'aspect de surface de l'une des formes 63 (fig. $62, n^{\circ} 1$ ) la ferait attribuer aux DS.P., sa pâte dans les cassures est extrêmement proche des communes (fig. 61, $n^{\circ} 3$ ), ce qui justifie le fait d'attirer l'attention sur cette particularité ou anomalie. Au contraire, le $\mathrm{n}^{\circ} 2$ de la figure 62 , que l'aspect de surface aussi bien que les cassures (fig. 61, $\mathrm{n}^{\circ}$ 6) caractérisent comme une DS.P. authentique, présente un profil inhabituel dans cette catégorie, et que l'on pourrait rapprocher de la forme de commune AB 36 par la section du rebord (type $\mathrm{A} 3$ ou $\mathrm{B} 3$ ?) et les dimensions.

Les récipients comportant un bec tubulaire ont presque toujours été retrouvés dans un état très fragmenté, mais la présence d'une anse opposée au bec est attestée sur trois formes presque complètes (Bonifay 1986, 216-219, fig. 1, $\mathrm{n}^{\circ} 1$ et 2 et fig. 2). A Gardanne, en DS.P., deux becs tubulaires appliqués sur des panses décorées (fig. 27) pourraient correspondre à la forme 36 . Sur le gros fragment de commune grise (fig. $51, \mathrm{n}^{\circ} 2$ ), le bec est collé à la lèvre, ce qui n'est pas le cas sur les DS.P. au Ve s., mais devient courant au VI ${ }^{\mathrm{e}}$ s. Il est remarquable qu'en Languedoc les deux exemplaires de La Lombarde soient aussi des productions qualifiées "d'intermédiaires" : l'un en pâte "granuleuse" - donc grossière - est décoré d'arceaux (Bonifay 1986, fig. 1, $\mathrm{n}^{\circ}$ 1) ; l'autre (Bonifay 1986, fig. 2), cuit en atmosphère oxydante, est considéré dans cette région comme une céramique commune. De même, on retrouve en Ligurie les formes à bec tubulaire en céramique commune rouge cuites selon ce même mode, attestées du IV au VI ${ }^{e}$ s. (Lamboglia 1950, fig. 85, 86 et 94 ; Gandolfi 1986 ; Olcese 1989, 212-213 et fig. 18).

En pâte de DS.P., le vase $n^{\circ} 3$ de la figure 62 peut être rapproché du type de la forme 36 , mais aussi des formes hémisphériques $\mathrm{O}$ de Saint-Blaise (Démians d'Archimbaud et al. à paraître, fig. 141, $\mathrm{n}^{\circ}$ 149-150).

Une forme non répertoriée dans l'une ou l'autre catćgorie (fig. 62, $n^{\circ} 4$ ), au façonnage fruste et dont la pâte grossière (fig. 61, $n^{\circ}$ ) pourrait la faire attribuer aux communes, est cependant décorée de guillochis, technique traditionnellement employée sur les céramiques fines. Un fond dont la section est normale parmi les DS.P., avec une paroi extérieure et la face inférieure soigneusement tournassées formant un pied annulaire (fig. $62, \mathrm{n}^{\circ} 5$ ), est en pâte véritablement commune. Un bord de coupelle (fig. $62, \mathrm{n}^{\circ} 6$ ) au profil répertorié en

Tabl. IX Répartition comparative des différentes formes (pour Saint-Blaise, toutes séquences $V e-V \mid e s$. confondues).

\begin{tabular}{|c|c|c|c|c|c|c|c|}
\hline Formes & A & B & D & E & F/K & Autres & Total \\
\hline GARDANNE & 219 & 75 & 23 & 81 & 14 & 18 & $430 \mathrm{ex}$. \\
$\%$ & 50,9 & 17,4 & 5,3 & 18,8 & 3,2 & 4,2 & \\
\hline SAINT-BLAISE & 208 & 100 & 20 & 25 & 16 & 32 & $401 \mathrm{ex}$. \\
$\%$ & 51,9 & 24,9 & 5 & 6,2 & 4 & 8 & \\
\hline
\end{tabular}




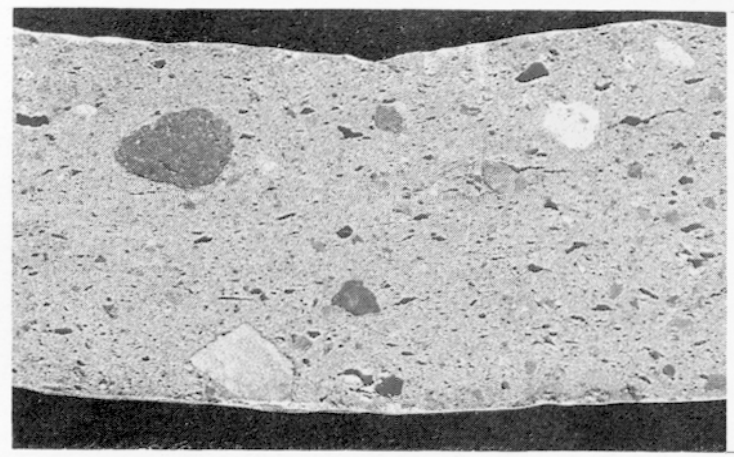

1
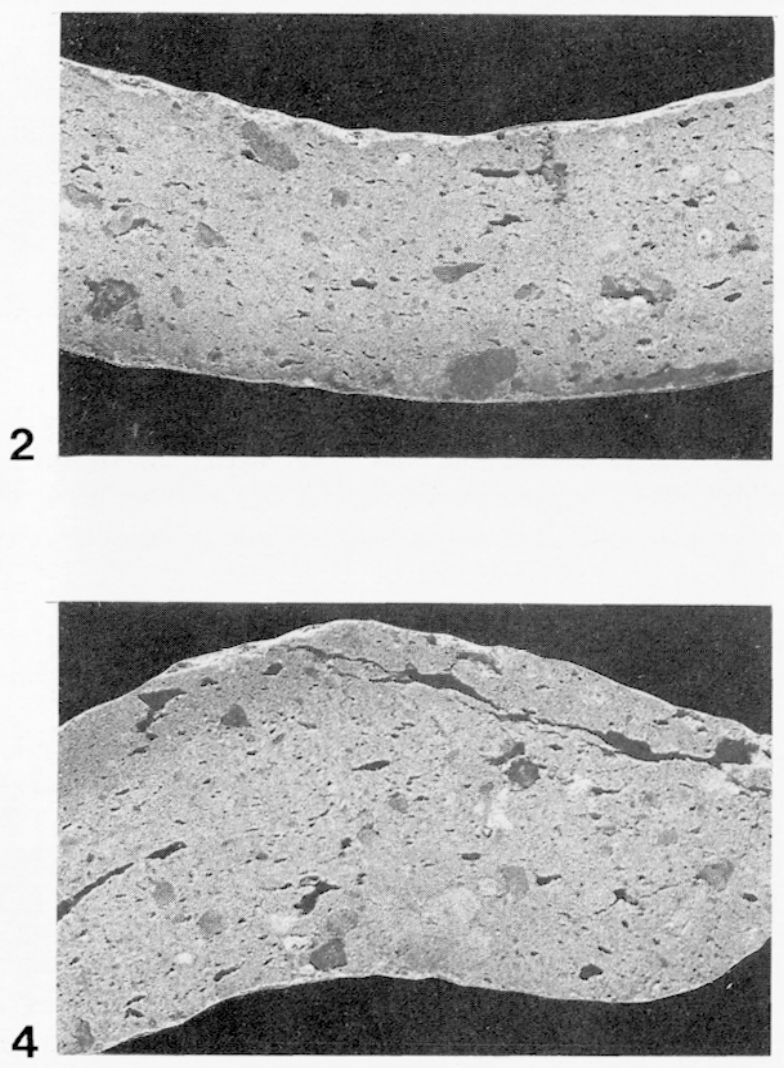
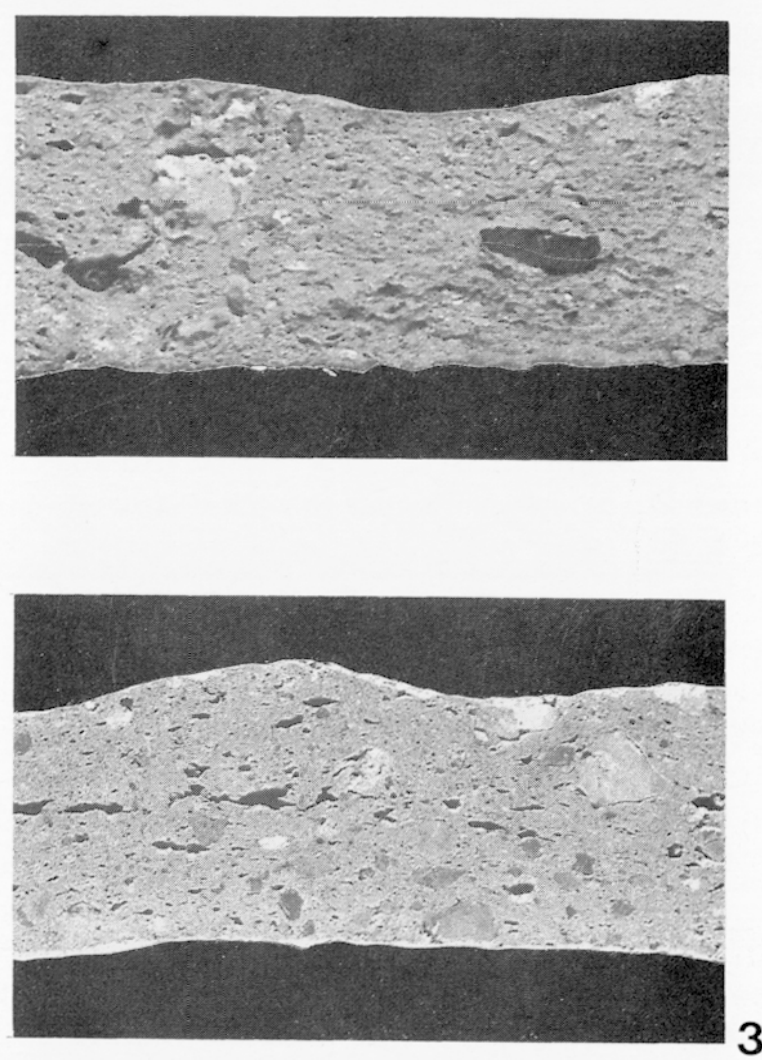

$\longleftarrow \mathrm{mm}$

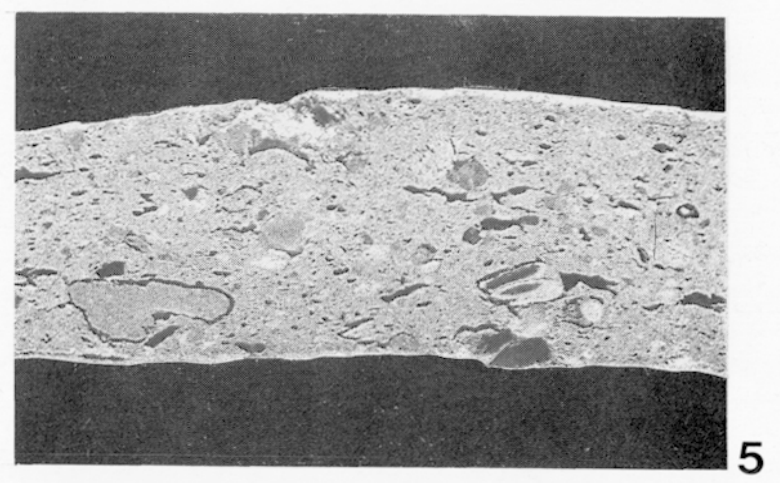

I 59 Commune grise. Exemples de pâtes assez fines et de dureté moyenne, très fréquentes.

DS.P. (forme 70) mais n'existant pas en céramique commune, est en pâte intermédiaire, avec des inclusions plus fines que les communes mais pourtant bien distincte des DS.P. (fig. 61, $\mathrm{n}^{\circ} 4$ ). Une forme peu fréquente en DS.P. (plus creuse que les assiettes et plus ouverte que les bols : fig. 15), a déjà été retrouvée à Âpt avec des caractères de pâte et de décor qui la situaient déjà à cheval sur les deux catégories (Kauffmann 1987 , fig. $11, \mathrm{n}^{\circ} 7$ et 8 ), et à Saint-Blaise en pâte grossière (Démians d'Archimbaud et al. à paraître, fig. 144, $\mathrm{n}^{\circ} 187$ ). Le fragment de Gardanne (fig. $62, \mathrm{n}^{\circ} 7$ ) est en pâte intermédiaire, mais toujours bien distincte des DS.P.

Pour mémoire, on observe le phénomène ponctuel de formes rares représentées dans les deux catégories. Ainsi pour la forme 26 (fig. 22) ou $\mathrm{K}$ (fig. 53), correspondant à des cruches à col étroit et allongé, à bord simple et à bec pincé. Le principe des couvercles ou bouchons à collerette adap- tables sur les cruches est comparable dans les deux catégories (en DS.P. : fig. $28, \mathrm{n}^{\circ} 223-224$; en commune : fig. $58, \mathrm{n}^{\circ} 1$ ). De même les jattes à panse conique ou "tians", formes ouvertes à large fond plat, existant en DS.P. (fig. 15) et non représentées en commune grise à Gardanne, mais connues sur d'autres sites, dans des niveaux de la première moitié du $\mathrm{V}^{\mathrm{e}}$ s. et répertoriées forme C (Aix-en-Provence : Guild 1980, 159, fig. 33b, $\mathrm{n}^{\circ} 2574$ et C.AT.H.M.A. 1986, 46, fig. 11, $\mathrm{n}^{\circ} 5$; Apt (Vaucluse) : Kauffmann 1987, fig. 11, n 5-6; Mormoiron (Vaucluse) : réunion C.A.T.H.M.A. du 4 avril 1991, compte-rendu dactylographié).

\subsubsection{Etude comparative des pâtes}

L'observation visuelle et tactile est une première étape pour l'appréciation de la structure et des 

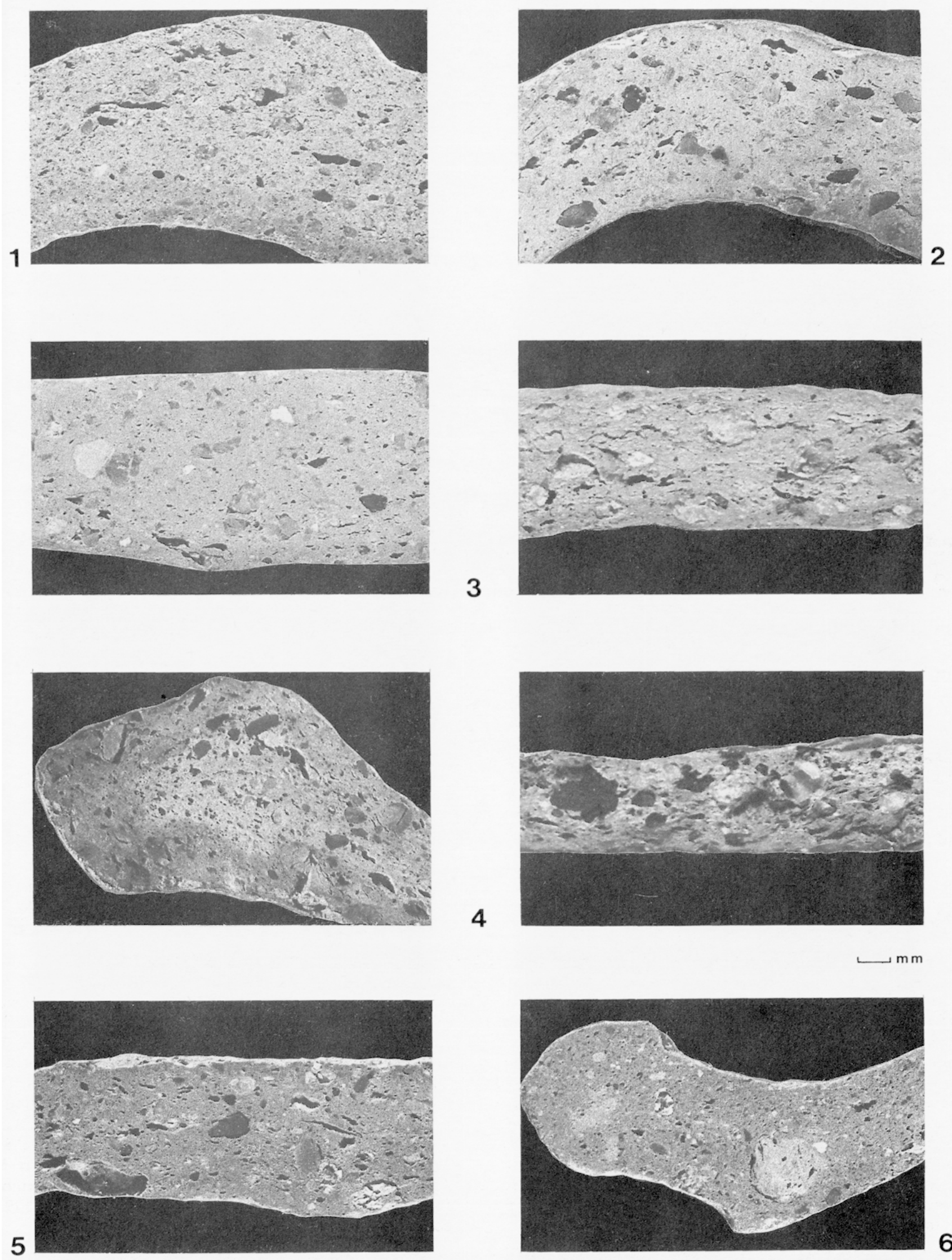

6

160 Commune grise. Exemples de pâtes assez grossières et assez dures, très fréquentes. 

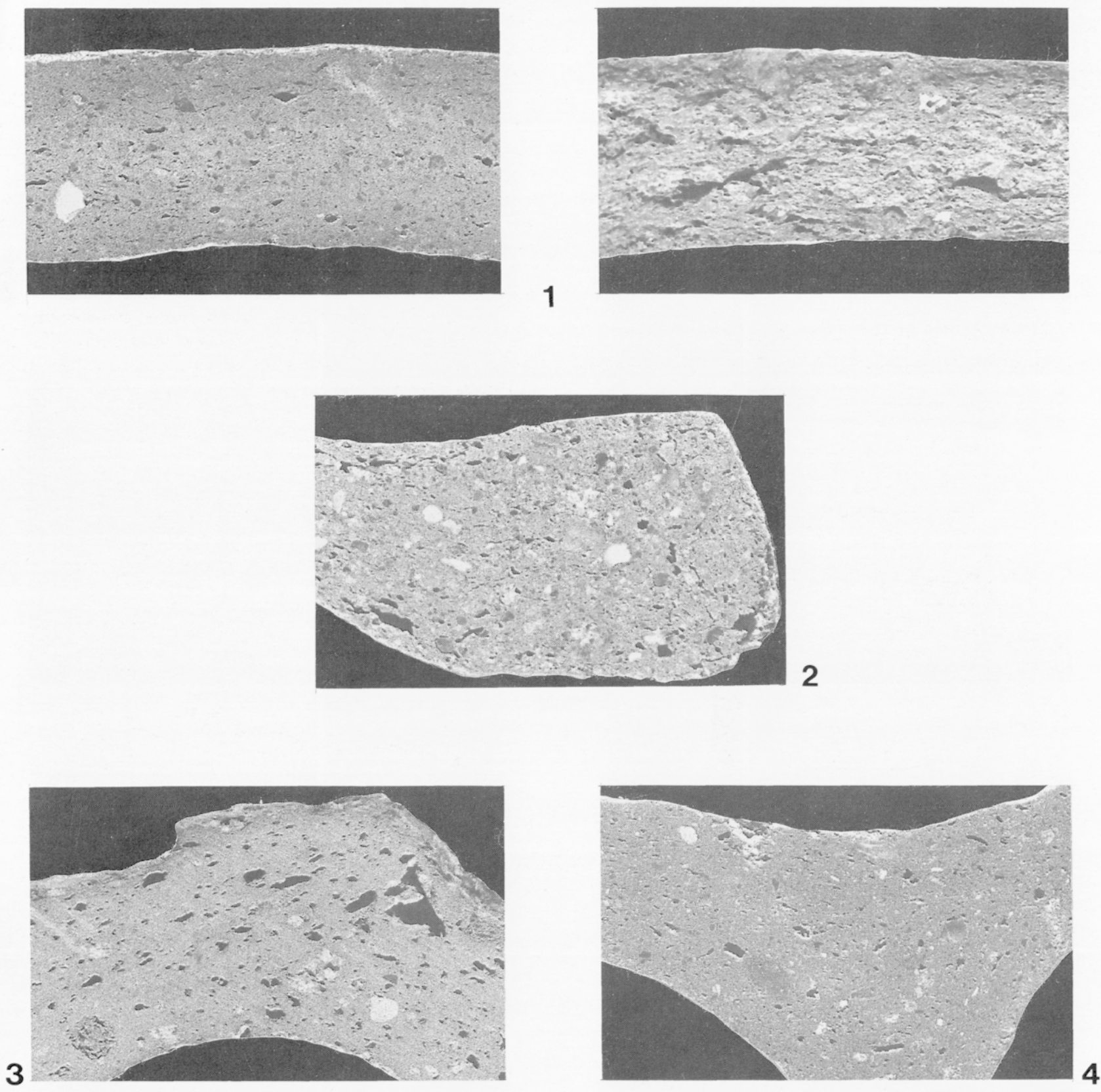

$\llcorner\mathrm{mm}$
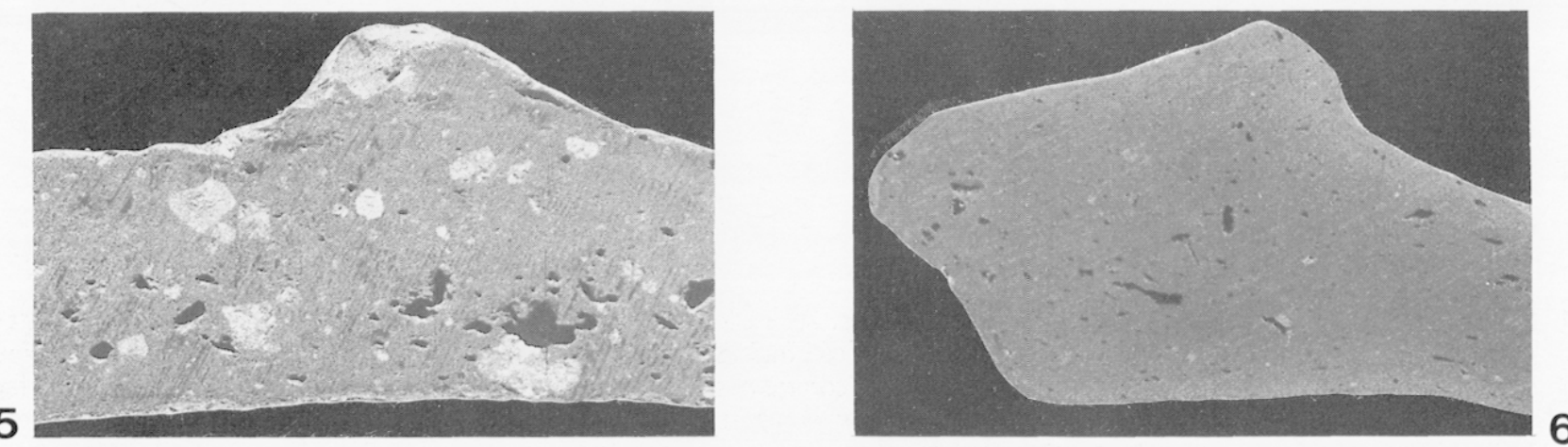

$161 N^{\circ} 1$ : commune "grise", pâte brun clair, fine, exceptionnelle ; 2 : commune "grise", pâte assez fine, granuleuse, dure, assez exceptionnelle ; 3 à 5 : pâtes intermédiaires entre les DS.P. et les communes ; 6 : pâte typique des DS.P. 
constituants des pâtes (fig. 59, 60, 61). Elle n'exige qu'un matériel sommaire, des connaissances limitées, et peut ainsi être facilement appliquée, comme par exemple le font nos collègues de l'Université de Barcelone (Buxeda 1992), parallèlement à des méthodes plus sophistiquées.

Pour notre matériel, des analyses géochimiques sont en cours (3). Les résultats seront présentés au $\mathrm{V}^{\mathrm{e}}$ colloque international sur la Céramique médiévale en Méditerranée occidentale, au Maroc, en novembre 1991.

Nous avons distingué ici trois catégories principales : communes grises, productions intermédiaires, et DS.P.

\section{Communes grises}

- Exemples de pâtes assez fines et de dureté moyenne, très fréquentes (fig. 59)

$-n^{\circ} 1$ (coupe et cassure) : forme B2 (fig. $46, n^{\circ} 1$ ). Surface douce. Texture serrée, vacuoles minuscules. Inclusions blanches, brunes, noires et transparentes ; les grossières sont rares, les fines nombreuses.

$-\mathrm{n}^{\circ} 2$ : forme A3 (fig. $39, \mathrm{n}^{\circ} 1$ ). Surface douce, légèrement micacée. Texture très serrée, vacuoles minuscules. Inclusions moyennes et fines, blanches et noires.

$-n^{\circ} 3$ : forme A2 (fig. $37, n^{\circ} 3$ ). Surface plutôt rugueuse, micacée. Texture serrée, vacuoles moyennes. Inclusions moyennes et fines, blanches, grises, brunes et transparentes. $-n^{\circ} 4$ : forme A2 (fig. $35, n^{\circ} 1$ ). Surface assez douce. Texture serrée, vacuoles allongées. Grosses inclusions blanches, noires, et transparentes fines.
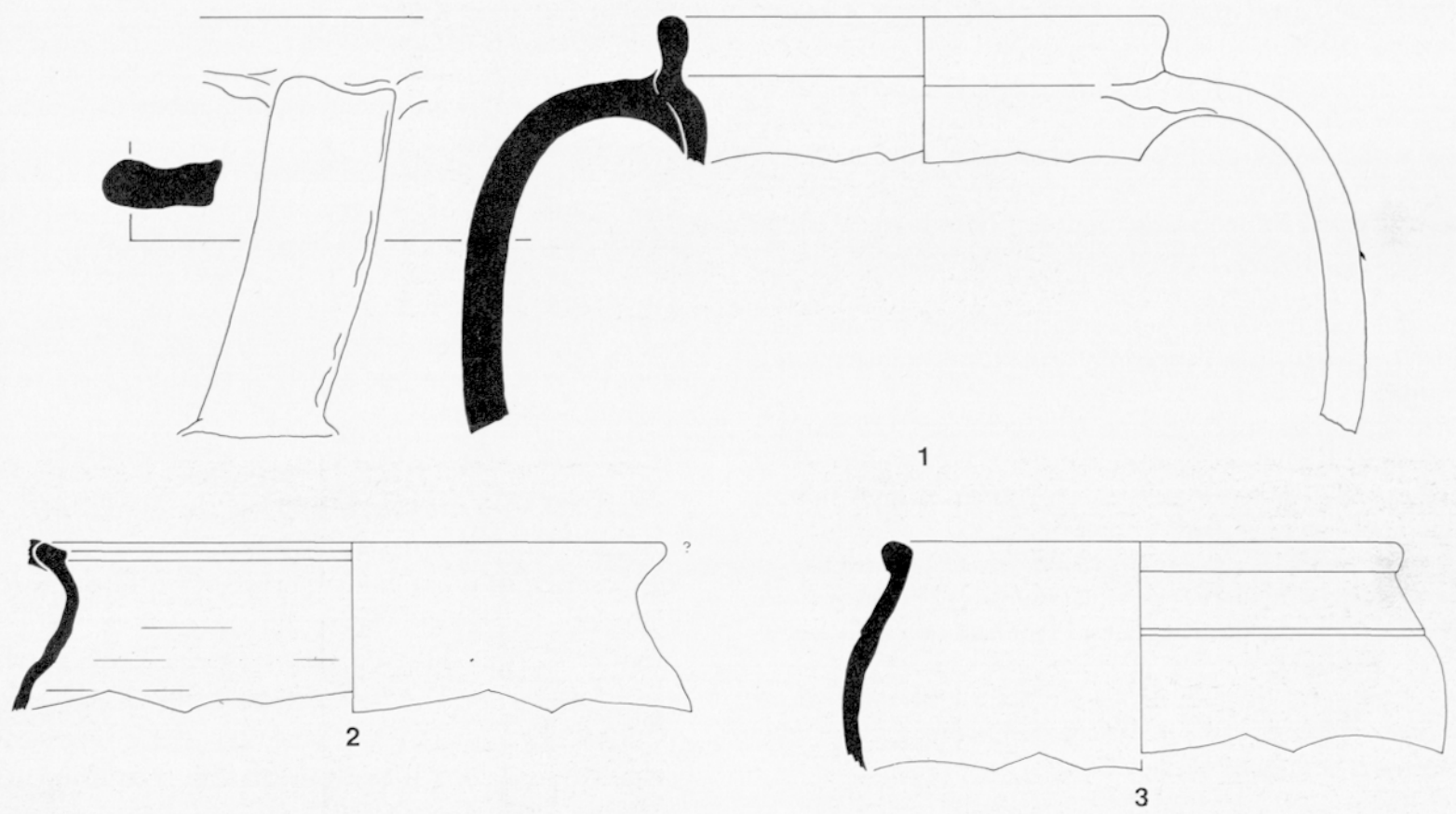

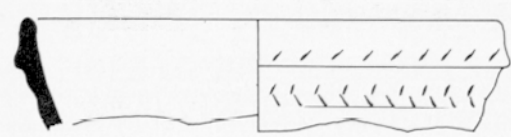

4

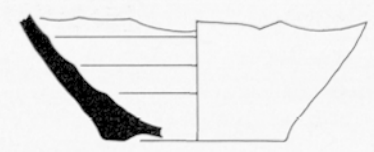

5
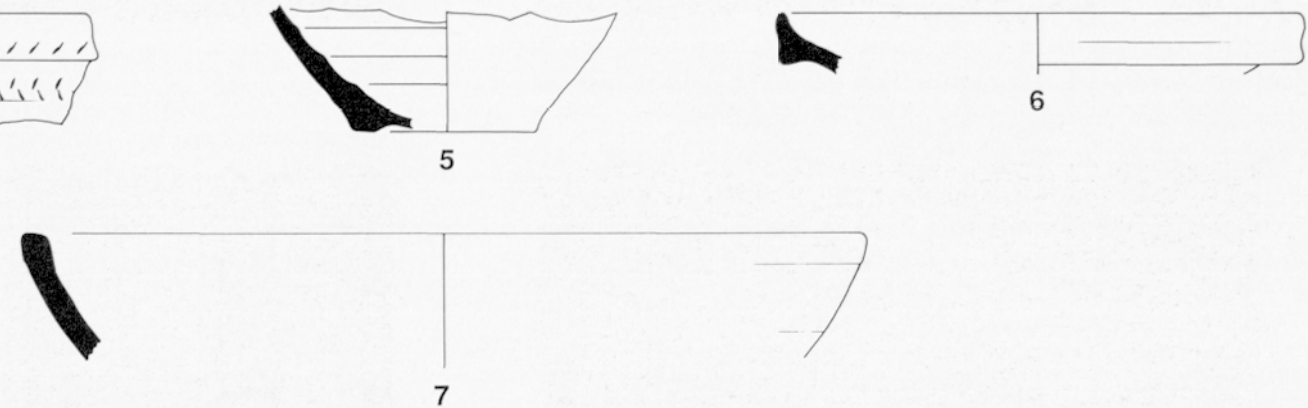

162 Exemples d'interférences entre les DS.P. et les communes. № 1 : forme 63 , aspect de surface type DS.P. et påte grossière dans les cassures (voir figure $61, n^{\circ} 3$ ) ; 2 : forme proche des communes $A B 36$, mais avec une pâte et un aspect de surface typique des DS.P. (voir figure $61, n^{\circ} 6$ ) ; 3 : forme proche des communes $O$ ou $A B 36$, mais avec une pâte et un aspect de surface typique des DS.P. ; 4 : forme exceptionnelle, non répertoriée, assez fine en surface mais grossière dans les cassures, décorée de guillochis (voir figure $\left.61, n^{\circ} 5\right) ; 5$ : fond de vase fermé (cruche ?), tournassé comme une céramique fine, mais en pâte commune comparable aux exemples présentés figure $59 ; 6$ : bord de coupelle forme 70 des DS.P., mais en pâte relativement grossière bien distincte de ces séries (voir figure $\left.61, n^{\circ} 4\right) ; 7$ : forme rare, existant uniquement avec des pátes intermédiaires, toujours distinctes des DS.P. comme des communes [éch. 1/3]. 


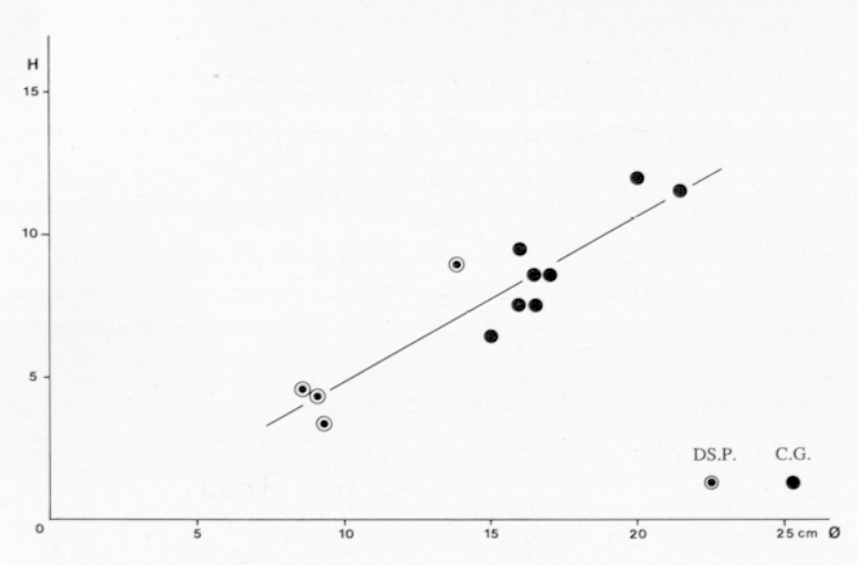

$-n^{\circ} 5$ : forme A1 (fig. $34, n^{\circ} 1$ ). Surface assez douce. Texture serrée, nombreuses vacuoles minuscules. Grosses inclusions blanches et grises, transparentes fines.

- Exemples de pâtes assez grossières et assez dures, très fréquentes (fig. 60)

$-n^{\circ} 1$ : forme A2 (fig. 37, $n^{\circ} 7$ ). Surface assez rugueuse, micacée. Texture assez serrée, vacuoles rares et minuscules. Nombreuses inclusions moyennes et fines, blanches et grises.

$-n^{\circ} 2$ : forme B3 (fig. 47, $n^{\circ} 4$ ). Surface granuleuse. Texture très serrée, pratiquement sans vacuoles. Nombreuses inclusions grossières et moyennes, noires et transparentes.

$-n^{\circ} 3$ (coupe et cassure) : forme A2A (fig. 38, $n^{\circ} 5$ ). Surface granuleuse. Texture serrée, petites vacuoles peu nombreuses. Nombreuses inclusions moyennes blanches et transparentes, rares noires.

$-n^{\circ} 4$ (coupe et cassure) : forme A3 (fig. 39, $n^{\circ} 12$ ). Surface granuleuse et rugueuse. Texture serrée, avec vacuoles. Très nombreuses inclusions grossières, moyennes et fines noires, rares grises et blanches grossières et fines.

$-n^{\circ} 5$ : forme A1 (fig. $34, n^{\circ} 14$ ). Surface granuleuse et rugueuse. Texture serrée, petites vacuoles. Nombreuses inclusions grossières et moyennes, blanches, noires et transparentes.

$-n^{\circ} 6$ : forme A2 (fig. $35, n^{\circ} 4$ ). Surface rugueuse. Texture très serrée, vacuoles rares. Grosses inclusions grises rares, fines nombreuses noires et blanches.

\section{- Exemples de pâtes "grises" exceptionnelles}

- Fig. 61, $\mathrm{n}^{\circ} 1$ (coupe et cassure) : forme A4A (fig. 41, $\mathrm{n}^{\circ} 5$ ). Pâte de couleur brun clair. Surface assez rugueuse, micacée. Texture serrée, avec quelques vacuoles. Inclusions moyennes blanches, moyennes et fines grises, et mica fin.

- Fig. 61, $\mathrm{n}^{\circ} 2$ : forme A1 (fig. $34, \mathrm{n}^{\circ} 8$ ). Surface assez rugueuse. Texture assez serrée, nombreuses petites vacuoles. Nombreuses inclusions moyennes et fines, blanches, grises, brunes et transparentes.
163 Comparaison des corrélations diamètres / hauteurs des bols DS.P. (formes $3,6,18$ ) et des formes B en commune. Les différents objets s'organisent autour d'un axe commun incliné d'environ $30^{\circ}$ par rapport à l'horizontale. La seule différence se trouve dans les dimensions plus réduites des bols en DS.P.

\section{Exemples de pâtes intermédiaires}

- Fig. 61, $\mathrm{n}^{\circ} 3$ : forme 63 (fig. 62, $\mathrm{n}^{\circ} 1$ ). Surface très douce. Texture très serrée, nombreuses vacuoles grosses, moyennes et petites. Rarcs grosscs inclusions blanches et noires, et inclusions blanches moyennes.

- Fig. 61, $\mathrm{n}^{\circ} 4$ : forme 70 (fig. 62, $\mathrm{n}^{\circ}$ 6). Surface corrodée. Texture serrée, petites vacuoles. Inclusions moyennes transparentes, blanches fines.

- Fig. $61, n^{\circ} 5$ : forme non répertoriée (fig. $62, n^{\circ} 4$ ). Surface très douce. Texture serrée, nombreuses vacuoles. Nombreuses inclusions blanches moyennes et fines.

\section{Exemple de pâte typique des DS.P.}

- Fig. 61, n० 6 : forme $A B 36$ ? (fig. 62, $n^{\circ}$ 2). Surface très douce. Texture serrée, rares petites vacuoles. Rares et minuscules inclusions grises.

Il ressort en premier lieu de ces observations que, si l'on écarte les inclusions, la texture de la pâte des communes est aussi serrée, et quelquefois plus, que celle des DS.P. Cette dernière est toujours similaire - à quelques variations près dans les dimensions des vacuoles - à l'exemple donné (fig. 61, $\left.n^{\circ} 6\right)$. Cela peut être attribué aux différences entre les constituants ou à la température de cuisson qui donnent un matériau plus sonore en commune grise. $\mathrm{La}$ distinction est donc facile entre les deux catégories.

Un rapport direct existe aussi entre l'aspect de surface et la pâte ; celui-là est d'autant plus granuleux que les inclusions sont grosses. Des distinctions apparaissent aussi dans la nature des sables. En multipliant ces observations, il sera peut-être possible de les mettre en relation avec des séries de formes ou

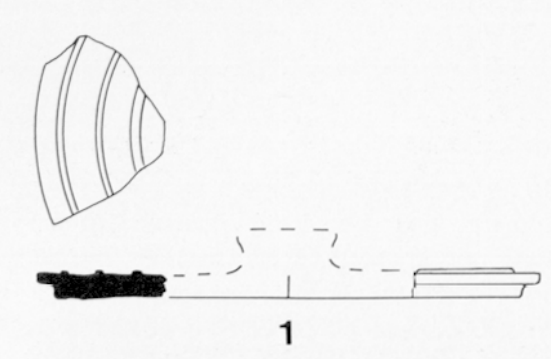

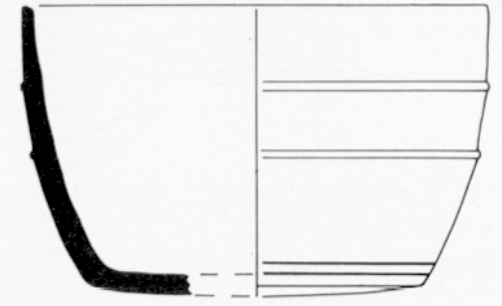

2

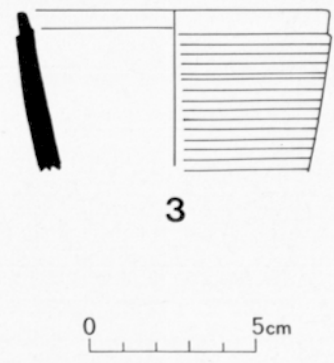

164 Pierre ollaire. № 1 : couvercle ; 2 : vase tronconique ; 3 : gobelet [éch. $1 / 3$ ]. 
des origines différentes, ce qui, dans ce lot, n'apparaît pas encore.

Les premières analyses géochimiques de commune grise effectuées sur cinq échantillons montrent une certaine diversité de composition qui tient à la nature même des kaolinites qui ne sont pas homogènes, avec cependant un faible taux de calcaire compris entre 1 et $7 \%$. Il s'agit toujours de pâtes siliceuses réfractaires kaolinitiques, mais qui ne se rattachent pas directement aux éléments régionaux déjà analysés (C.A.T.H.M.A. 1986, 50) : cela déjà pourrait autoriser à les considérer comme un groupe particulier.

Contrairement à ce que laisseraient penser les exemplaires que nous qualifions d'intermédiaires, il semble qu'il n'y ait en aucun cas de pont entre les communes et les DS.P. En effet, la pâte de ces dernières comporte un pourcentage de chaux toujours plus élevé, de l'ordre de 8 à $20 \%$.

Les recherches en cours sur les poteries et les gisements d'argile de la région marseillaise et des environs du massif de l'Etoile devront permettre de préciser ces questions, car il paraît possible de cerner les affleurements kaolinitiques de cette zone, et par là même de mieux situer les aires de production encore imparfaitement localisées.

\section{Les autres mobiliers}

\subsection{LES OBJETS EN PIERRE OLLAIRE}

Quatre récipients différents en matériau non argileux complètent le vaisselier culinaire en pâte grise siliceuse résistant au feu. Trouvé en petit nombre, ce matériel lithique (fig. 64) est un produit marginal qui est souvent associé aux céramiques de l'Antiquité tardive dans la plupart des fouilles provençales. Dans l'aire transalpine, il est abondant.

Un couvercle $\left(n^{\circ} 1\right)$, un petit vase tronconique $\left(n^{\circ} 2\right)$ et un gobelet $\left(n^{\circ} 3\right)$ auxquels $s^{\prime}$ ajoute un fond, ont en commun une texture très fine de couleur gris-verdâtre, et un tournage très soigné. D'autre part, ces formes étonnent par leur petite dimension et la faible épaisseur des parois qui présentent à l'intérieur des traces de tournage. A l'extérieur, des sillons en creux réguliers forment décor dans le cas du gobelet. Des petits bourrelets espacés régulièrement scandent les parois du vase tronconique et du couvercle qui s'y adapte parfaitement.

C'est dans le matériel ligure et du Tessin que l'on retrouve des répliques exactes de ces petites
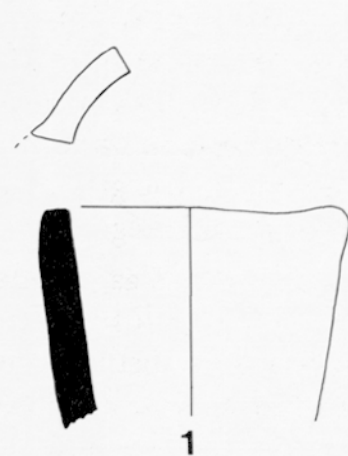

1

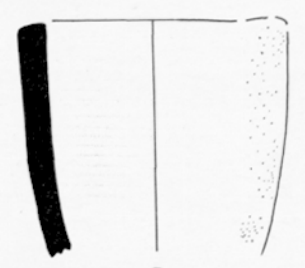

6
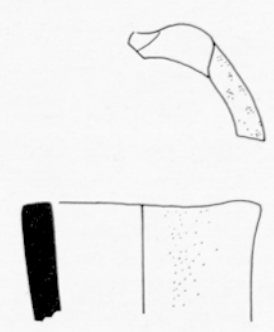

2

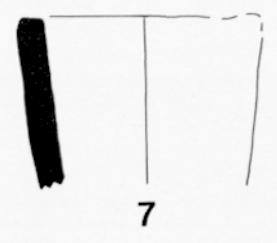

7

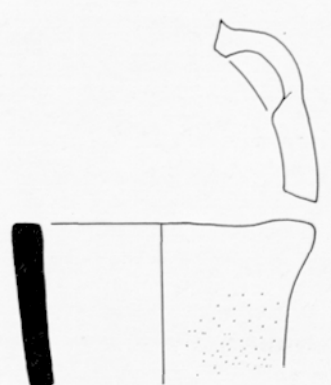

3

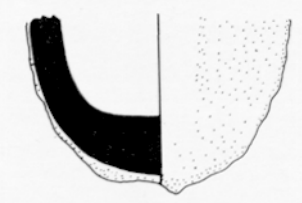

8

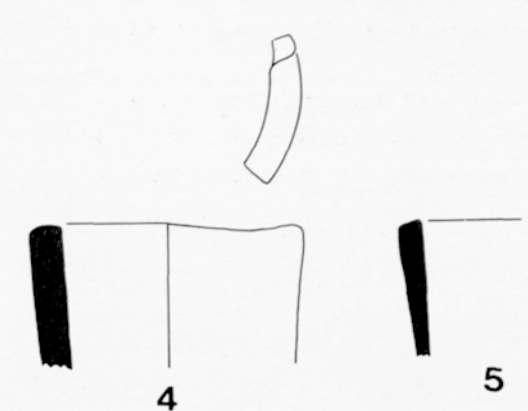

4

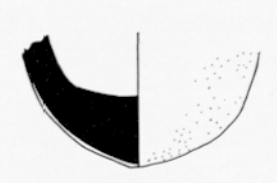

9

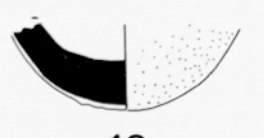

10

165 Creusets de bronziers. NN 1 à 4 : rebords avec bec verseur ; 5 à $7:$ fragments de rebords ; 8 à 10 : fonds ovoïdes avec coulées d'oxydes [éch. 1/3]. 

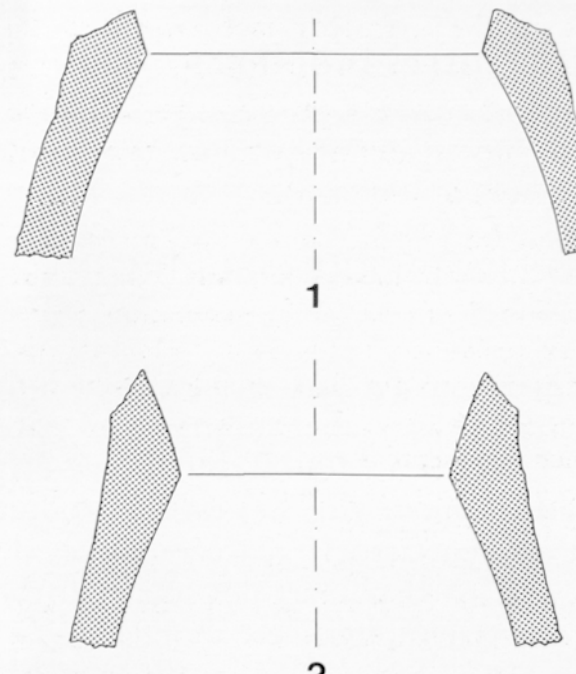

2

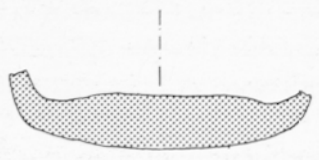

5

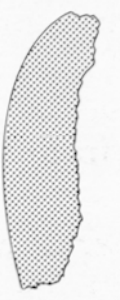

8
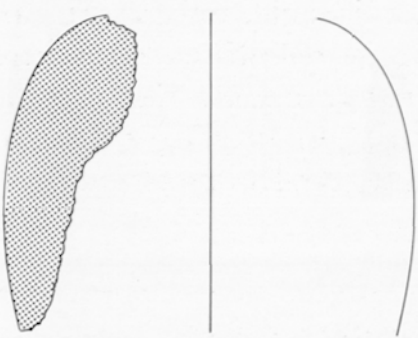

11

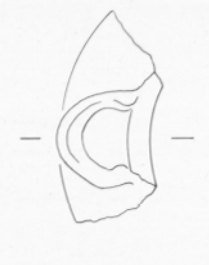

3

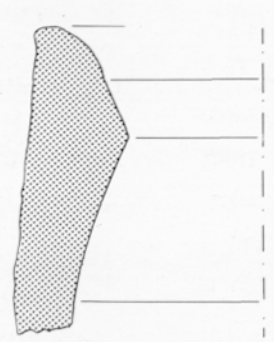

A

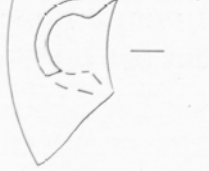

4
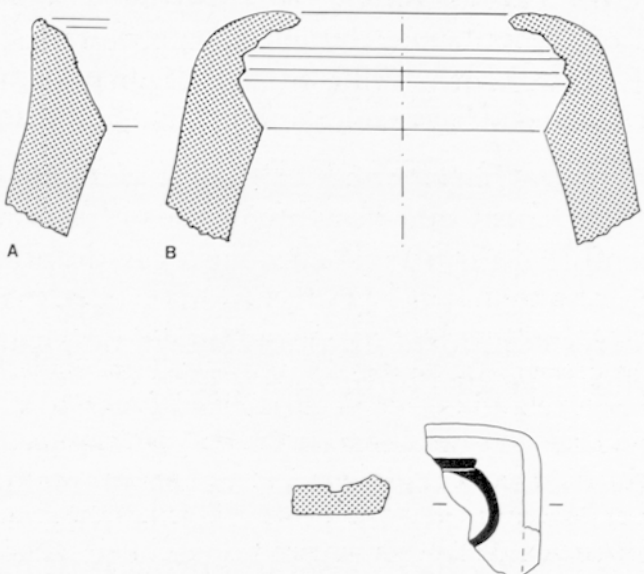

7
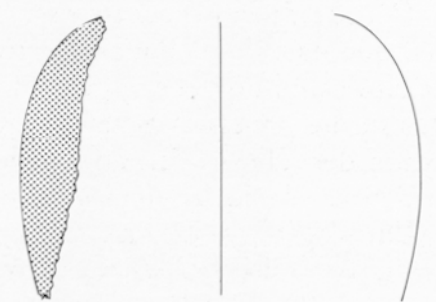

10
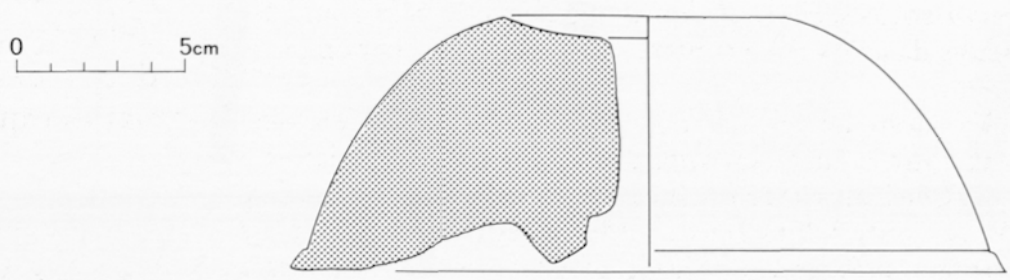

12

166 Moules de fonderie de bronze. № 1 à 4 : éléments externes bitronconiques ; 5 et $6:$ disques ?; $7:$ boucle de ceinture ; 8 à 11 : éléments internes ovoïdes ; 12 : élément interne hémisphérique [éch. 1/3].

formes de vase, verre et couvercle peu documentées en Provence (Pfeifer 1986 ; Murialdo 1986 ; La pietra olla- re... 1986 ; La pietra ollare... 1987 ; Gandolfi 1986 ; Démians d'Archimbaud et al. à paraître, fig. 86-87). 


\subsection{LE TRAVAIL DU BRONZE}

Quarante-deux fragments de creusets et cent trente-huit fragments de moules témoignent d'un artisanat de bronziers d'une certaine importance.

\subsubsection{Les creusets}

Les creusets (fig. 65) sont façonnés en argile réfractaire siliceuse, granuleuse, surcuite et grésée. Ils ne présentent aucune trace de tournage. L'intérieur est lissé par la coulée du métal en fusion. L'extérieur présente des traces de métal, des coulures de vitrification des crasses et d'oxydes de cuivre rouge et vert sur les lèvres et les fonds.

La forme est légèrement tronconique, presque cylindrique, avec un fond ovoüde. Les parois dont l'épaisseur est à peu près constante $(8 \mathrm{à} 12 \mathrm{~mm})$ n'ont pas de lèvre marquée, mais le rebord est simplement aplati. Par contre, les becs verseurs sont soigneusement réalisés par écrasement de la largeur d'un pouce. Le diamètre est de 8 à $10 \mathrm{~cm}$, la hauteur peut être estimée à une dizaine de $\mathrm{cm}$, ce qui implique une contenance de $250 \mathrm{~cm}^{3}$ environ, soit $2 \mathrm{~kg}$ de métal.

On dénombre 7 fonds et 14 rebords dont 4 comportent un bec verseur.

Analyse des creusets

L'étude de ces creusets fera l'objet d'une publication spécifique par M.-C. Bailly-Maître, mais d'ores et déjà on peut être certain qu'elle apportera des informations intéressantes sur les pratiques métallurgiques, en particulier la composition des alliages. En effet, selon la teneur en étain, les propriétés mécaniques du métal varient et la fonte en dépend.

L'analyse chimique en cours se fait dans le cadre du programme Artémise-scories et produits associés (4). Elle permettra de définir les éléments majeurs et les éléments traces contenus dans les coulures vertes et les vitrifications qui couvrent les parois des creusets, ainsi que l'imprégnation du métal au sein de l'argile réfractaire qui les compose.

Ces objets ayant été découverts dans une fosse-dépotoir, il n'est malheureusement pas possible de connaître les installations associées. Seuls quelques fragments de bronze ont été recueillis dans le même contexte. Ils seront étudiés comparativement.

La nature de ces objets est assez identique aux creusets mis au jour à Fort-Harrouard à Sorel-Moussel (Eure-etLoire) (Queixalos 1987). L'horizon chronologique bien daté $\mathrm{du} V^{e} s$. ajoute à l'intérêt de la découverte, car les résultats pourront s'intégrer à une étude plus large de la métallurgie du bronze (5), notamment à partir des résidus de creusets dont la typologie est en cours de constitution (6).

\subsubsection{Les moules}

Les très nombreux morceaux de moules (fig. 66) qui à première vue paraissaient informes se sont révélés fort intéressants à l'examen. Ils sont en terre légère, très poreuse et mêlée de restes végétaux visibles à l'état de traces en négatif. On serait tenté de rappeler que, pour la fabrication des moules à cloches, les anciennes méthodes préconisent l'adjonction de fumier de cheval afin de réduire les problèmes posés par le retrait de la forme lors du séchage. Cela entraîne une porosité qui pourrait aussi faciliter l'évacuation des gaz lors de la coulée du métal en l'absence d'évent (voir notamment Donati 1981 et Cat. expo. 1987, 38).

La technique de fonte à la cire perdue implique la destruction des moules après refroidissement, afin d'en extraire les objets, ce qui explique leur extrême fragmentation. Seulement 25 morceaux présentent des restes de formes. Quelques-uns correspondent à des objets simples, disques (?) et boucle de ceinture (fig. 66, $\mathrm{n}^{\circ} 5-7$ ). Les autres appartiennent à deux séries bien représentées :

- partie femelle : moule externe, de forme bitronconique avec parfois orifice de coulage du métal $\left(n^{\circ} 1-4\right)$;

- partie mâle : moule interne, de forme ovoïde ( $\left.n^{\circ} 8-11\right)$.

A ces séries s'ajoute un objet unique : un gros fragment hémisphérique, moule interne ou matrice, avec un ressaut pour le rebord à la base et un orifice central de section carrée dans sa partie supérieure, trace de l'axe lors de la fabrication de la forme $\left(\mathrm{n}^{\circ} 12\right)$ ?

Ces éléments mâles et femelles ne semblent pas s'emboîter, ou constituer les deux parties du moule d'un même objet. Ils sont à attribuer à des moules de pièces de mobilier dont la tôle devait être épaisse de 2 à $3 \mathrm{~mm}$. De tels éléments sont connus pour la période romaine, notamment au Maroc (Boucher 1971 ; Boube-Piccot 1975, 7 à 25 et pl. 1, 6, 7, 47, 50, 108, 144 ; Tassinari 1975 ; Boucher, Tassinari 1976), et il s'agit très vraisemblablement du même type de pièces de candélabres et aussi d'éléments de jambages de lit, hauts d'une dizaine de centimètres pour un diamètre de 10 à $20 \mathrm{~cm}$, destinés à être emboîtés sur des axes de bois.

Les restes métalliques en rapport avec cet artisanat sont rares : seulement trois petits fragments de bronze scoriacé.

Le travail du fer est aussi attesté par une dizaine de scories de forge (7), mais cela est tout à fait normal dans le cadre d'une villa. Quelques dizaines de clous, de morceaux de ferrures et de fers à cheval, des fragments rouillés ainsi que de petits débris ou objets de bronze (dont une épingle, un anneau, une tige plate, des morceaux de tôle fine), constituent les déchets que l'on trouve habituellement dans les dépotoirs d'habitat. Pour mémoire, il faut aussi signaler quelques petites coulées de plomb.

\subsection{LE MOBILIER EN VERRE}

Outre la vaisselle en terre, la fosse contenait un lot de verres. Celui-ci bien, que d'importance numérique modeste (160 fragments), est cependant doublement intéressant : d'une part son homogénéité nous autorise à avancer une datation assez précise, d'autre part la présence de quelques déchets et surtout de 3 fragments de céramique vitrifiés, identifiés comme des creusets, permet de penser que les verres retrouvés, ou du moins une partie d'entre eux, sont d'origine locale. 

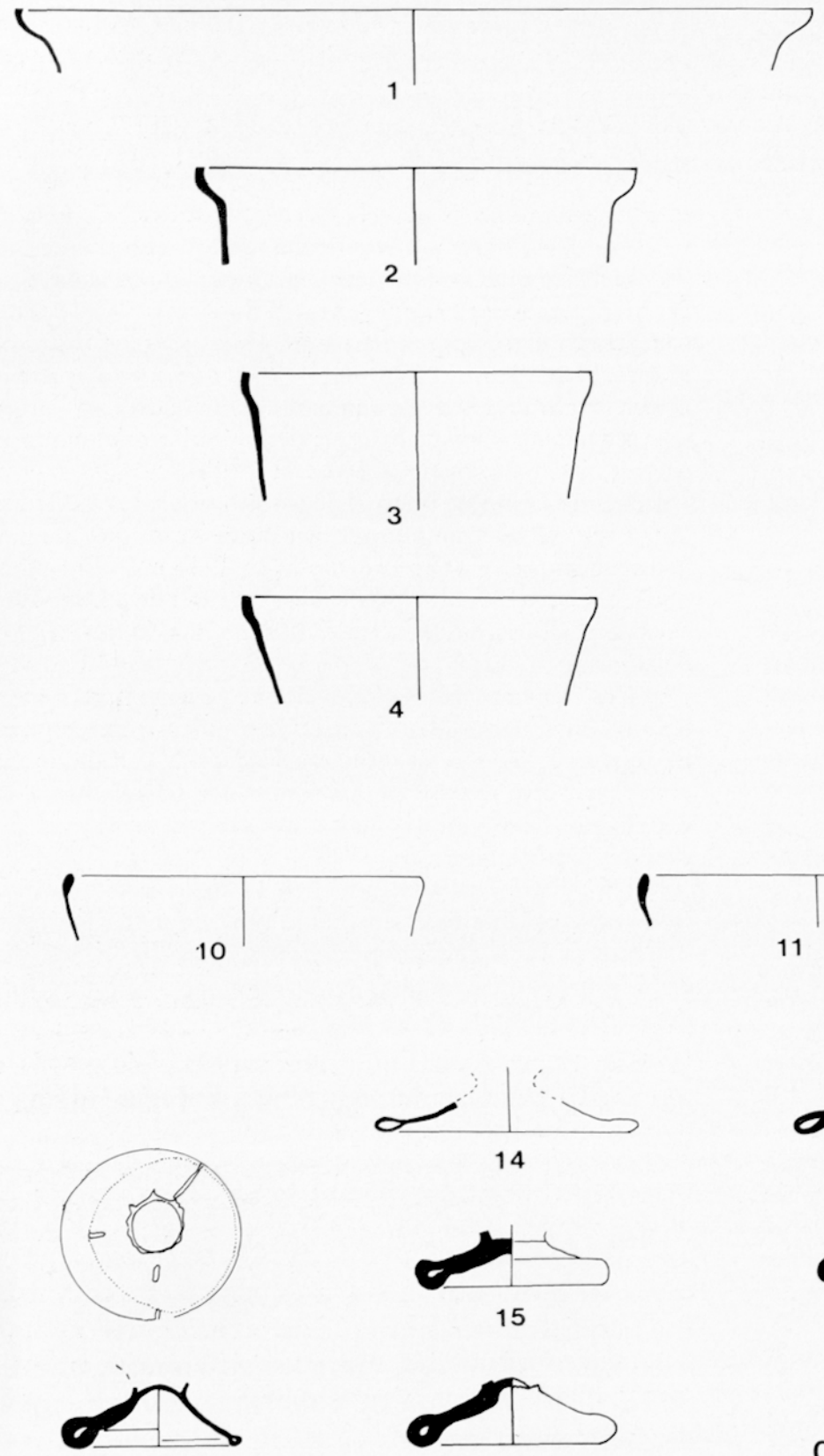

13
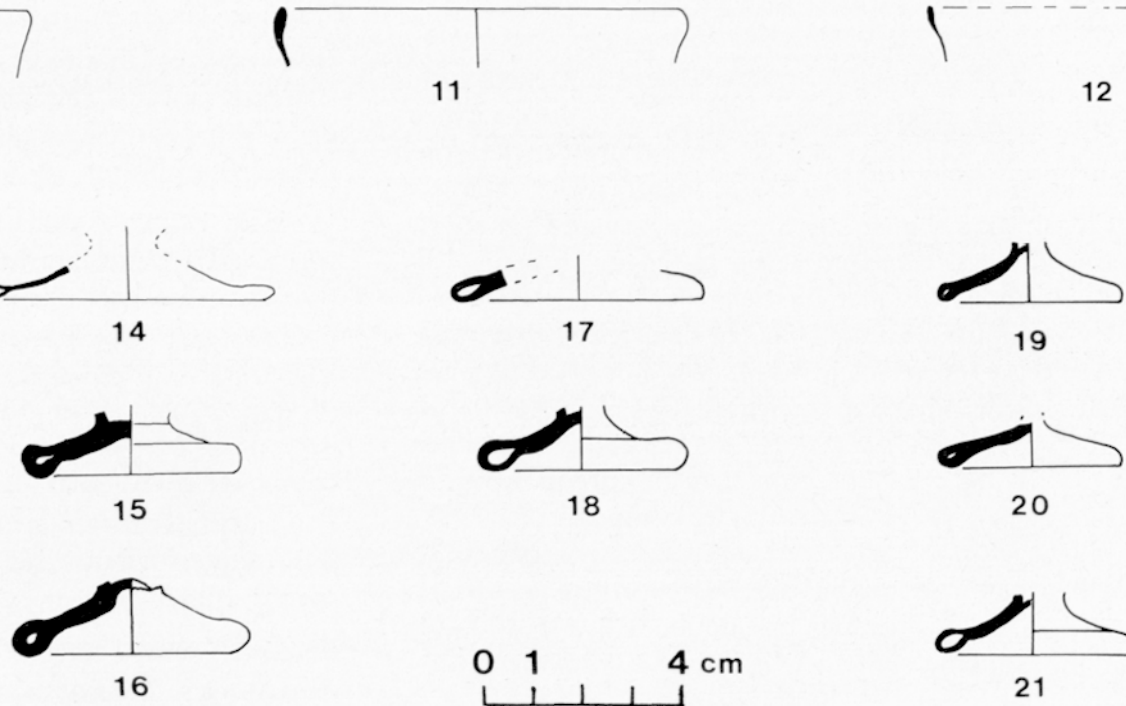

19

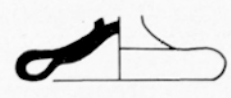

18
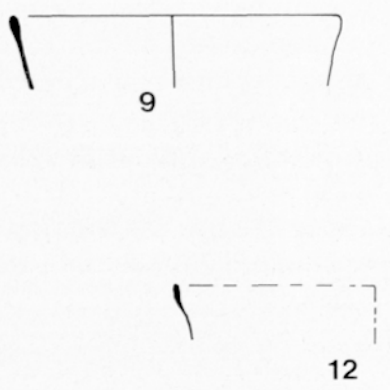

12
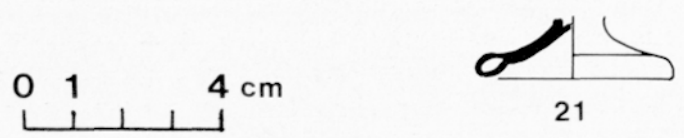

21

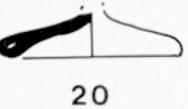

0
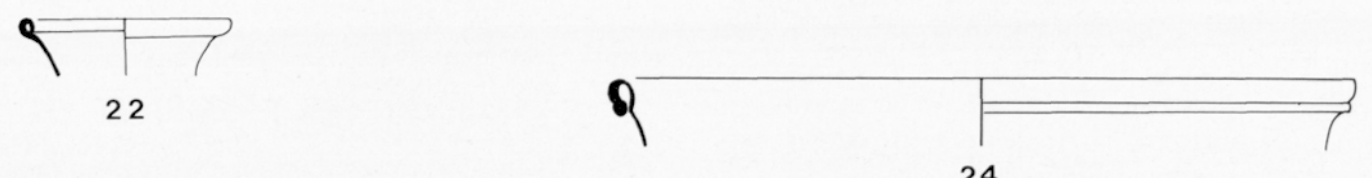

24

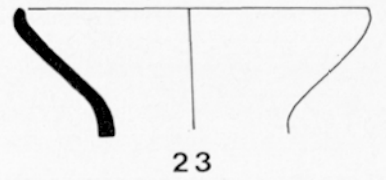

$a^{-}$ 
L'étude a porté sur une centaine de fragments ; un tiers du matériel comprenant surtout des éléments de paroi atypiques a donc été écarté. Il est difficile de comptabiliser le nombre d'objets à partir de la documentation sélectionnée. Il y a un minimum de 33 récipients. Les creusets de verrier en céramique sont représentés par 3 tessons dont 2 seulement proviennent du même contexte que les verres. Mais le fragment de fond trouvé hors de la fosse (fig. 69, $\mathrm{n}^{\circ}$ 64) appartient très vraisemblablement au même ensemble et peut-être à la même pièce que le rebord issu de la fosse (fig. 69, $\mathrm{n}^{\circ}$ 62).

\subsubsection{Inventaire et interprétations}

\section{- Verres à rebord sans lèvre}

Les pièces les plus anciennes sont probablement les rebords à arête vive, sans lèvre, de 4 récipients (fig. 67). Trois font partie de verres à boire (gobelet ou verre à pied); ils sont de teinte vert clair $\left(n^{\circ} 3,4\right)$ ou plus sombre, olivâtre $\left(n^{\circ} 2\right)$. Le rebord le plus large $(180 \mathrm{~mm})$ est celui d'une coupelle; le verre très fin, presque incolore, est bleuté $\left(n^{\circ} 1\right)$.

Ces rebords, façonnés rapidement sans que l'on ait pris le soin d'épaissir l'extrémité pour former une lèvre, sont très communs dans tout l'Empire au IVe et encore dans la première moitié du $\mathrm{V}^{\mathrm{e}} \mathrm{s}$. ; ils peuvent appartenir à des formes diverses : coupelles, bols, verres à pied ou gobelets. Il n'est pas rare d'en retrouver aux époques suivantes, mais alors ils ne sont plus prépondérants et dans bien des cas doivent être considérés comme résiduels. L'étude des verreries exhumées sur divers sites languedociens et provençaux du $\mathrm{IV}^{\mathbf{e}}$ et $\mathrm{du}$ $\mathrm{V}^{\mathrm{e}} \mathrm{s}$., permet aussi quelques remarques quant à la teinte de la matière. Au IVe s. le verre clair verdâtre, bleuté ou presque incolore semble le plus fréquent alors qu'au $\mathrm{V}^{\mathrm{e}} \mathrm{s}$. domine la teinte olivâtre, couleur qui d'ailleurs n'est pas propre à ces objets : elle se retrouve dans de nombreuses formes, en particulier sur des cruches.

Ces observations résultent essentiellement des études faites d'une part sur les verres du IVe s. trouvés à Lunel-Viel (Raynaud 1990) et à Arles (recherches en cours), et d'autre part sur le matériel exhumé des fouilles de la basilique du Clos de La Lombarde à Narbonne dans un contexte daté des premières décennies du $\mathrm{V}^{\mathrm{e}} \mathrm{s}$. (Foy 1991) et sur les verres du chantier de la Bourse à Marseille venant de niveaux attribuables à la période 1 , correspondant à la dcuxic̀me moitié du $V^{e}$ s. et plus précisément - les études ultérieures le soulignent - au second quart du Ve s. (Foy 1987, 291-294).

\section{$\checkmark$ Verres à pied conique et verres à tige}

Des petits pieds coniques formés d'une double épaisseur de verre sont au nombre de 6 ou 7 (fig. 67). Ils sont soufflés dans une matière bleutée $\left(\mathrm{n}^{\circ} 16,17,18,14\right)$ ou vert clair $\left(n^{\circ} 13,15\right)$. L'un d'eux $\left(n^{\circ} 13\right)$ a la particularité d'avoir 3 petits segments de fils de verre posés à égale distance sur la face supérieure. Il est impossible de dire s'il s'agit d'un reste de décor ou d'un accident dû à la maladresse de l'artisan ; l'irrégularité de ce verre au pourtour tantôt épais, tantôt mince, plaide plutôt en faveur de la seconde hypothèse. Ces pieds pourraient être associés aux rebords à lèvre arrondie, de même teinte $\left(n^{\circ} 5,9,10\right)$, mais il n'est pas possible de restituer le profil de ces verres en raison de la taille réduite des tessons.
Ce même mauvais état de conservation ne permet pas toujours de distinguer sans problème ces pieds, appartenant sans doute à une variante du type 109 de Isings, des supports des verres à tige (Isings 111) plus tardifs. Les premiers sont en effet reconnus habituellement dans les contextes du $\mathrm{V}^{\mathrm{e}}$ s., surtout dans la deuxième moitié du siècle (Foy 1987, $n^{\circ}$ 56-58) alors que le verre à tige (Isings 111), exceptionnel dans la deuxième partie du Ve s., ne semble vraiment utilisé qu'au VIe s.

Ce sont bien évidemment les proportions de chacun des types qui nous autorisent à avancer des datations. Ainsi les fouilles de Marseille, relativement riches en vaisselle de verre, montrent que le verre à tige succède immédiatement au verre à pied conique, les deux étant utilisés concurremment pendant une courte période : la première moitié du VIe $\mathrm{s}$. Ensuite le verre à tige devient le récipient à boire le plus commun dans les pays méditerranéens durant la deuxième moitié du $\mathrm{VI}^{e}$ et tout le VII $\mathrm{s}$. au moins. En Provence, l'étude du matériel des fouilles de Marseille, de Saint-Blaise (Foy à paraître) et de Toulon (Bérato 1986) atteste bien la prépondérance de cette forme à cette époque. A Gardanne, nous reconnaisssons deux pieds de verre à tige vert jaunâtre ( $n^{\circ} 19$ et 20 ), un troisième $\left(n^{\circ} 21\right)$, incolore peut-être, mais plus incertainement, identifié aussi à un verre à tige. Quelques rebords jaunâtres $\left(n^{\circ} 6,7,8\right.$ et 11$)$ doivent être associés à ces disques de verre qui portaient des tiges.

\section{$\checkmark$ Goulots}

Deux fragments d'embouchure étroite faisaient partie de goulots (fig. 67). L'un, épais et olivâtre ( $n^{\circ} 23$ ), est comparable à une pièce de la Bourse datée du deuxième quart du $V^{e} s$. (Foy 1987, $n^{\circ} 27$ ) mais ici le rebord à lèvre arrondie et non pas à arête vive comme à Marseille pourrait être un peu plus récent. Le second, en verre fin, de teinte très légèrement verdâtre $\left(n^{\circ} 22\right)$, est, par sa matière, beaucoup plus proche des verreries décorées de filets de verre blanc opaque (voir fig. 68 , $\left.n^{\circ} 37\right)$. Son rebord est replié en dedans.

Cet élément réduit interdit toute attribution certaine à un type connu. Notons seulement que deux bouteilles dans la typologie de Isings ont un rebord rabattu à l'intérieur (forme 101) ou à l'extérieur (forme 133), cette dernière étant la plus tardive. Des bouteilles de formes diverses dont le goulot se termine par un rebord ourlé ont été anciennement trouvées à Karanis (Harden 1936, pl. XVII à XIX) et à Rome dans un contexte fin IVe-début $V^{e} s$. (Sternini 1989, $n^{\circ} 55$ ).

\section{- Coupes à rebord ourlé}

Deux rebords de coupes (fig. 67), incolore ( $\left.n^{\circ} 24\right)$ et bleuté $\left(n^{\circ} 25\right)$, sont aussi ourlés mais cette fois-ci à l'extérieur; ils se rattachent sans doute à la forme 115 de Isings ou à un type proche. Mais ces bords peuvent appartenir à des pièces différentes, ce qui expliquerait leur datation incertaine.

Ils sont signalés entre autres à Tarragone (Benet 1989, $n^{\circ}$ 9-17) et à Narbonne (Foy 1991, fig. 6) dans des contextes du début du $\mathrm{V}^{e} \mathrm{~s}$., à Rome dans un niveau du milieu du $\mathrm{V}^{e} \mathrm{~s}$. (Sternini 1989, $n^{\circ}$ 5). A Saint-Blaise (Foy à paraître, fig. 159, $\left.n^{\circ} 123\right)$, ce rebord est daté du début du VI ${ }^{e}$ s., et dans les fouilles de Tours, ces bords nombreux se situent entre la deuxième moitié du VII et le milieu du VII ${ }^{e}$ s. (Motteau 1985, $\mathrm{n}^{\circ} 116$ à 120).

\section{- Verreries à décor émaillé}

Les diverses formes partiellement décrites ci-dessus ne sont représentées que par un petit nombre d'exemplaires : 2 bouteilles non comparables, 2 bols ou coupelles au rebord ourlé, 4 pièces au bord sans lèvre, 2 à 4 verres à tige et 5 à 7 verres à pied conique. 

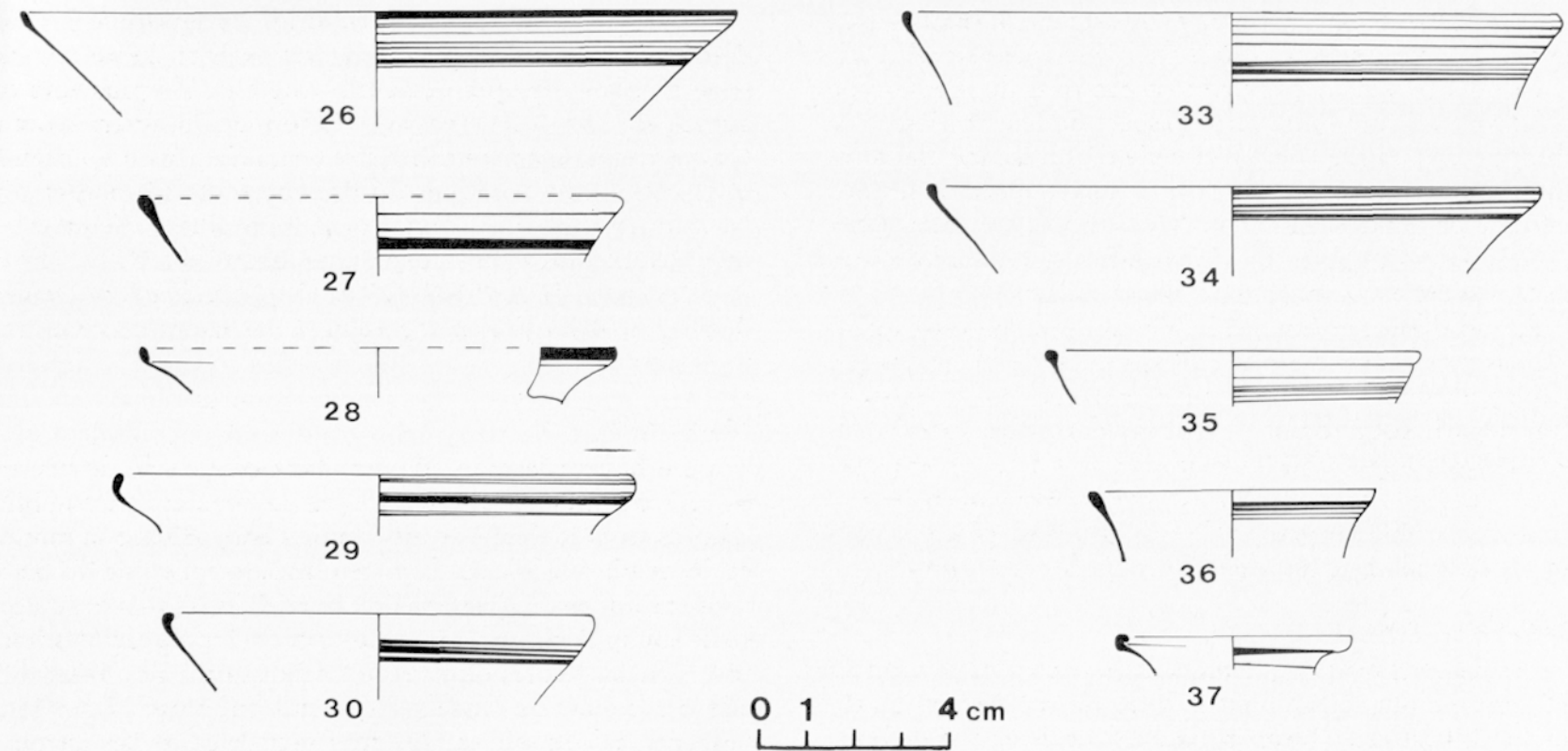

35
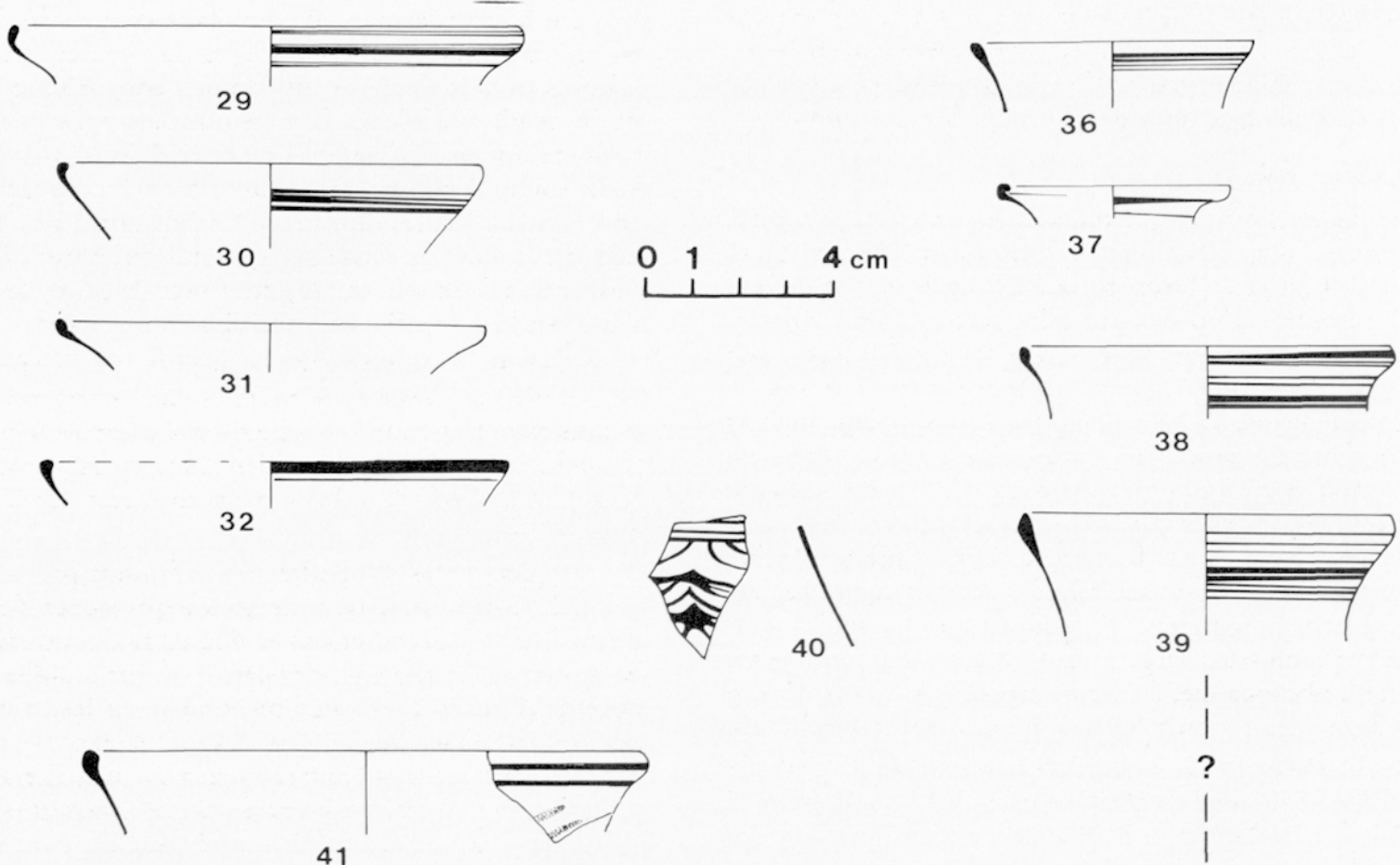

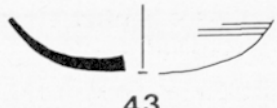

43
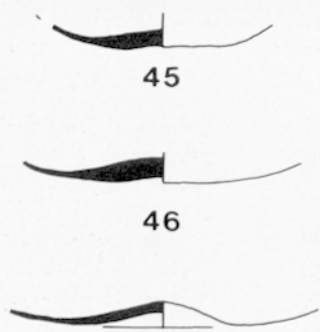

47

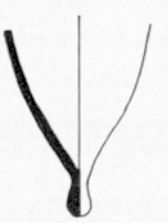

49

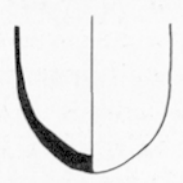

51

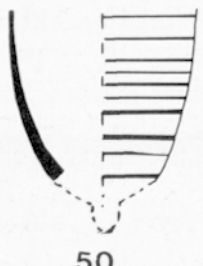

50

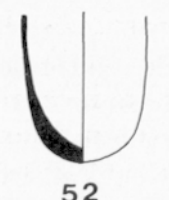

52

D $68 \mathrm{~N}^{\circ} 26$ à 32, 35 à 43, 50 : verres à décor rapporté blanc opaque ; 33 et 34 : à décor bleu ; 49 à 52 : lampes [éch. 1/2]. 
Le matériel le plus abondant consiste en un ensemble de fragments de verres souvent très fins, de teinte vert pâle, irisés. Ces débris qui ne proviennent pas tous du même type d'objet, ont en commun, outre l'aspect de leur matière, une technique décorative (fig. 68) : celle d'application de verre blanc opaque sous forme de cordons horizontaux, de festons $\left(n^{\circ} 40\right)$ ou de gouttes. Les filets d'émail blanc ont été rapportés sur la paroi des objets à peine soufflés et se sont incrustés dans la matière encore incomplètement solidifiée.

Aujourd'hui ce décor est le plus souvent émoussé quant il n'a pas totalement disparu : les fils de verre blanc opaque enroulés plusieurs fois autour des embouchures n'existent qu'en négatif sous forme de sillons parallèles au rebord. Nous avons comptabilisé 14 rebords ayant cette ornementation $\left(n^{\circ} 32\right.$ à $\left.39 ; n^{\circ} 41,42\right)$ un fragment de paroi $\left(n^{\circ} 40\right)$ et 4 fonds. Le profil et le gabarit de ces tessons suggèrent la présence de bouteilles, de gobelets, de coupelles et de lampes.

\section{- Bouteilles et gobelets}

Les deux rebords les plus modestes atteignent environ 50 et $60 \mathrm{~mm}$. Le plus étroit dont la lèvre est repliée vers l'intérieur ( $\left.n^{\circ} 37\right)$ est probablement un élément de goulot de bouteille. L'autre ( $\left.\mathrm{n}^{\circ} 36\right)$ peut aussi faire partie d'une forme fermée ou bien d'un gobelet tout comme les rebords légèrement plus larges, d'environ $80 \mathrm{~mm}$ de diamètre ( $\mathrm{n}^{\circ} 35,38$ et 39 ).

Nous ignorons la forme générale de ces objets. Doit-on les assimiler aux verres campaniformes à panse carénée et fond terminé par un bouton, tels ceux qui ont été fréquemment exhumés des nécropoles mérovingiennes ? Ce rapprochement est tentant dans la mesure où la matière de teinte vert d'eau et le profil de ces rebords sont comparables aux exemplaires mérovingiens. Il faudrait alors associer à ces embouchures un fond large à profil d'accolade, terminé par un petit bouton opaque, de même aspect que les fils déposés en spirale sur les rebords $\left(n^{\circ} 48\right)$. La double ornementation émaillée est largement connue depuis fort longtemps dans les trouvailles des cimetières mérovingiens (à titre d'exemple on peut citer les découvertes des nécropoles de Trivières, Ciply, Maine Saint-Paul ; Faider-Feytmans 1940, ${ }^{\circ}$ 27, 48, 49, $52 . .$.$) . Ces verreries sont datées habituellement de la première$ moitié du VIe $s$.

\section{- Lampes?}

Un autre bouton terminal émaillé est visible sur un fond de profil différent du précédent $\left(n^{\circ} 49\right)$. Conique, très effilé, ce fond est peut-être celui d'une lampe ou bien celui d'une petite amphore de verre au corps cylindrique tout à fait différente de celle répertoriée par Isings (forme 60). Les amphores en verre tubulaires, rappelant par leurs proportions les spatheion en terre cuite, ont été reconnues dans des fouilles à Ephèse (Czurda-Ruth 1989, $\mathrm{n}^{\circ} 70$ ) et Tarragone (Benet 1989, $n^{\circ}$ 9-47), mais ces deux trouvailles n'ont aucune ornementation émaillée. La pièce tarragonnaise datée du début du $\mathrm{V}^{\mathrm{e}} \mathrm{s}$. porte un décor caractéristique de cette période : des figures géométriques obtenues par une gravure superficielle, sorte d'abrasion. Dans les fouilles de la Bourse, les fonds à bouton terminal identifiés comme des lampes se trouvent à la fois dans des niveaux du début du VI ${ }^{e}$ s. (Foy 1984, $n^{\circ} 73$ ) et du début du VII ${ }^{\circ}$ s. (Ibid., $n^{\circ} 111,112$ et 113).

Deux autres fonds, cette fois-ci tubulaires, à l'extrémité arrondie, doivent être considérés comme des éléments de cornets servant de vases à boire ou plus vraisemblablement de luminaire ( $\mathrm{n}^{\circ} 50$ et 51 ). Des fonds comparables ont été recueillis dans les fouilles de Marseille sur des sols du VII $s$. (Foy 1984, $\mathrm{n}^{\circ} 139$ ).
Une pièce très fragmentée, dont on ignore à la fois le diamètre et la terminaison, a la particularité d'être couverte d'un fil blanc opaque serpentiforme enroulé plusieurs fois autour de son corps $\left(n^{\circ} 50\right)$. Cette pièce pouvait avoir un fond arrondi ou au contraire être terminée par un bouton. En effet, des fonds coniques décorés de filets d'émail et d'un bouton terminal sont connus dans les fouilles de Saint-Blaise où ils sont datés de la première moitié du VI ${ }^{\mathrm{e}} \mathrm{s}$. (Foy à paraître $b$, fig. $160, n^{\circ} 128,129$ et 132). Aussi ce fond de Gardanne peut-il aussi bien appartenir à une lampe qu'à une petite amphore.

\section{- Coupelles}

Le bol ou la coupelle est, dans ce groupe de verrerie ornées de filets d'émail, le type le plus fréquent. Neuf rebords dont le diamètre est compris entre 90 et $168 \mathrm{~mm}$ ont ce décor bien visible sur la lèvre ou au dessous ; les fils blancs forment un faisceau de parallèles très fines ou un cordon plus épais souligné par une ligne plus mince. La plupart des bords sont rentrants et les lèvres sont toujours épaissies. Il est difficile d'associer des fonds à ces embouchures. En effet, à l'exception d'un seul $\left(n^{\circ} 43\right)$, les fonds de coupelles retrouvés n'ont pas le décor d'émail spécifique ( ${ }^{\circ} 44$ à 47).

A cette série de rebords, il convient d'ajouter deux autres pièces, évasées, de même taille et en verre fin mais ornées cette fois-ci, non pas de fils blancs, mais d'applications serpentiformes colorées en bleu; elles sont analogues à un fragment trouvé à la Bourse dans un niveau du VII ${ }^{e} s$. (Foy $\left.1984, n^{\circ} 96\right)$.

Le décor des coupelles ne se limitait pas toujours au rebord. Un petit tesson de paroi qui porte des réseaux d'émail $\left(n^{\circ} 40\right)$ laisse imaginer une verrerie enrichie de guirlandes émaillées à la base des parois ou sur toute la surface de l'objet, à l'instar de ce que l'on connaît sur les trouvailles des nécropoles du nord de la Gaule. A titre d'exemple, nous pourrons mentionner les découvertes anciennes de Trivières où les pièces plus récemment exhumées dans le cimetière de Vieuxville (Alenus-Lecerf 1986, fig. 5) et datées du début du VI $s$.

Quelquefois, deux procédés décoratifs coexistent sur le même objet. Nous en avons pour preuve deux rebords munis de filets d'émail et de côtes inclinées obtenues au moule ( $n^{\circ} 41$ et 42 ). Ce décor tronqué suffit cependant pour deviner l'aspect originel de ces coupes : les nervures obliques devaient créer un relief de stries sur toute la paroi.

Les fragments de verre ornés d'émail sont connus dans le midi de la France principalement sur les sites de la Bourse, de Saint-Blaise et de Nîmes. A Marseille, ce décor qui apparaît dans le second quart du $V^{e} s$. représente à cette époque seulement $3 \%$ de la verrerie. Il n'est pas plus abondant dans la seconde moitié du siècle. En revanche, il est présent sur plus de $50 \%$ des verres au début du siècle suivant, pour disparaître presque totalement dans la deuxième partie du VI ${ }^{e} s$. (environ $6 \%$ des verres). Les résultats de l'étude des verres de Saint-Blaise (Foy à paraitre) sont moins significatifs quant à la chronologie de ce décor : en effet il se répartit presque équitablement entre la première et la deuxième moitié du $\mathrm{VI}^{e}$ s. Il faut cependant tenir compte ici des multiples remaniements de ce site, qui expliquent peut-être l'abondance de cette ornementation émaillée encore à la fin du $\mathrm{VI}^{e} \mathrm{~s}$. A Nîmes, la verrerie trouvée dans les fouilles de la rue Sauve n'est pas très importante (Raynaud 1990, fig. 128). Il est cependant intéressant de noter, dans un contexte daté par l'auteur de la fin du $V^{e}$ ou plutôt de la première moitié du $\mathrm{VI}^{e}$ s., la présence de pieds coniques et de rebords émaillés. 


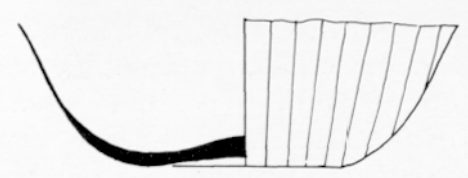

52

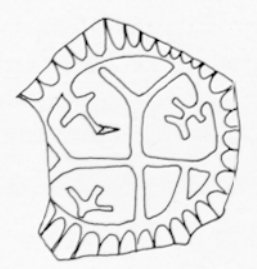

55

$9^{-}$

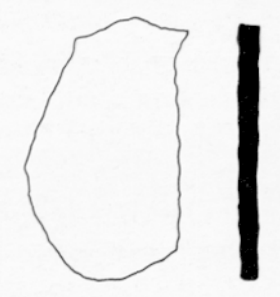

56

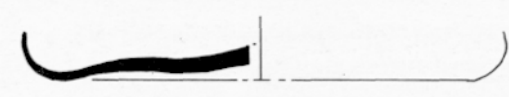

58

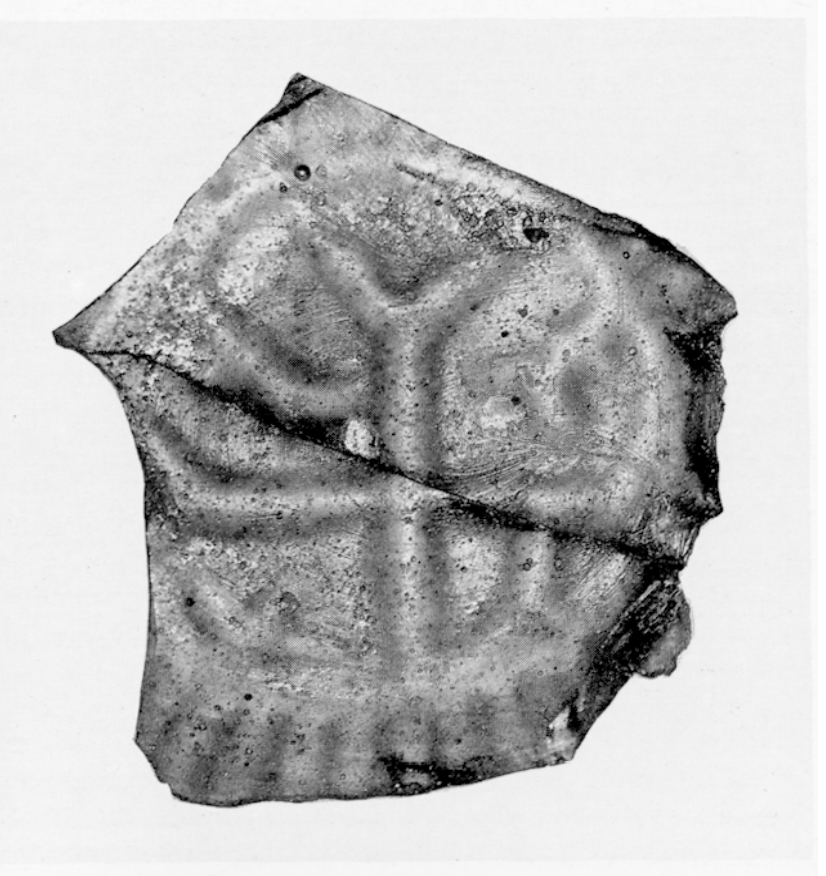

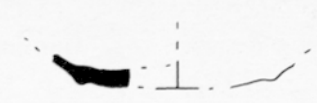

53
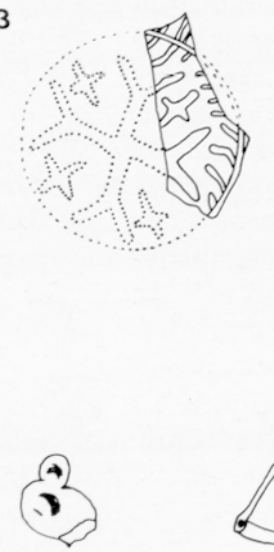

60

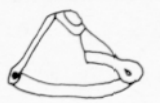

61
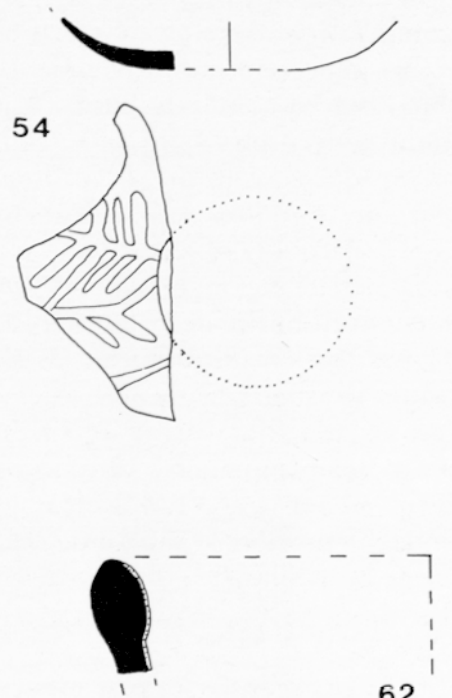

62

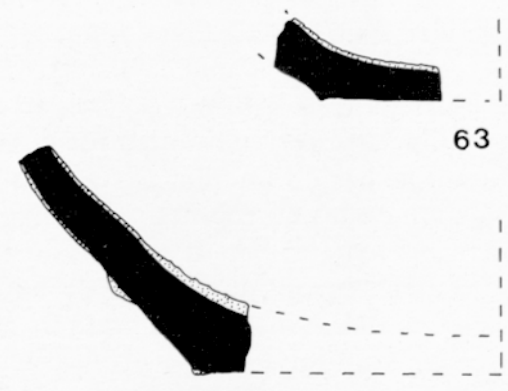

64

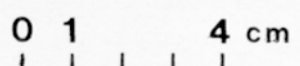

59

$\triangle 69 N^{\circ} 52$ et 53 : fonds de verres à décor chrétien; 60 et 61 : déchets de verre ; 62 à 64 : creusets vitrifiés [éch. 1/2].

70 Fond de verre à décor chrétien (voir figure 69, $n^{\circ} 52$ ).

\section{- Coupelles à décor chrétien}

Il n'est pas certain que la distinction entre coupelles à décor d'émail rapporté et coupelles à décor chrétien moulé soit pertinente. En effet les deux fragments précédents (fig. $68, \mathrm{n}^{\circ} 41,42$ ) montrent bien que les deux techniques ornementales (moulage et application à chaud) peuvent figurer sur le même objet ; ainsi il n'est pas impossible que les coupes dont le fond portait un décor chrétien aient aussi des fils d'émail sous le rebord. 
Deux pièces au moins, probablement trois (fig. 69), ont été soufflées dans un moule qui a imprimé un décor sous le fond et sur la totalité, ou seulement la base des parois. La coupelle la plus complète est de teinte vert d'eau ( $n^{\circ} 52$ et fig. 70). Le diamètre du fond atteint $45 \mathrm{~mm}$ et les parois sont conservées sur $30 \mathrm{~mm}$. L'évasement de la partie supérieure semble indiquer la proximité du rebord. L'objet complet ne devait guère excéder $40 \mathrm{~mm}$ de hauteur, et le diamètre de l'ouverture devait être de l'ordre de $110 \mathrm{~mm}$. Sous le fond, à l'intérieur du médaillon circulaire, on observe une croix dont 3 des extrémités sont fourchées. En revanche il est plus difficile de distinguer entre les 4 branches les petites figures qui pourraient être interprétées comme des croisettes mal imprimées. L'estampage est en effet de mauvaise qualité : la croix est décentrée et de ce fait une des extrémités a été tronquée ; quant aux croisettes, elles sont en partie confondues avec la bordure du médaillon. A l'extérieur du cercle qui limite le décor chrétien, rayonnent environ 36 côtes serrées qui remontent, verticales sur les parois de la coupe.

Le second fond $\left(n^{\circ} 53\right)$ beaucoup plus fragmenté est verdâtre comme le précédent. A l'intérieur du médaillon apparaît un $\mathrm{V}$ en relief que nous interprétons comme la fourche du bout d'une branche d'une grande croix ; nous y voyons aussi la dent de l'extrémité d'un autre bras de la croix et, entre les deux, une croisette. Nous restituons donc un schéma décoratif assez proche du fond précédent. En revanche, le décor ornemental périphérique est différent : ce ne sont plus des côtes mais, disposés en échelle de part et d'autre du pourtour du médaillon, des feuillages.

C'est aussi un décor de feuillages stylisés qui est moulé sur un fragment situé à la base de la paroi d'une coupe, juste à la jonction avec le fond ( $\left.n^{\circ} 54\right)$. Nous ne savons rien de l'ornementation imprimée sur le fond.

Ce n'est pas la première fois que l'on découvre en Provence, dans des contextes de la fin de l'Antiquité, des fonds de verrerie portant un décor chrétien moulé. Les trouvailles de Gardanne ( 2 ou 3 pièces) s'ajoutent aux 5 pièces marseillaises, aux 7 ou 8 verreries de Saint-Blaise, à l'exemplaire de Toulon (Bérato 1986, fig. $15, \mathrm{n}^{\circ} 78$ ) et au fond inédit provenant d'anciennes fouilles sur l'oppidum de Constantine (8).

On comptabilise donc, à ce jour, au minimum 16 fonds de coupelle à décor chrétien en Provence. Les symboles imprimés sont des chrismes ou des croix de différents modèles. Le chrisme a été identifié sur deux pièces très fragmentées exhumées du chantier de la Bourse (Foy 1977, pl. IV, $\mathrm{n}^{\circ} 28 ; 1987, \mathrm{n}^{\circ} 79$ ).

Une croix unique, à 4 branches, est moulée sur 4 pièces. Ces croix pattées ou aux extrémités arrondies sont le plus souvent cantonnées de quatre globules. Sur les autres fonds, la croix principale est flanquée de croisettes, et parmi ceux-ci domine le schéma de la croix aux branches fines et fourchées contenant à l'intérieur de celles-ci des croisettes de même type : 4 pièces au décor correspondant à cette dernière description proviennent de Saint-Blaise (Foy à paraître $b$, fig. 157 et $158, n^{\circ} 101$ à 104) et le motif du fond de Gardanne $\mathrm{n}^{\circ} 52$, en dépit de sa mauvaise impression semble devoir s'apparenter à ce modèle. Il est plus difficile d'y rattacher le fond $n^{\circ} 53$ du fait de son extrême fragmentation.

Tous les fonds provençaux proviennent de fouilles d'habitat. Les excavations anciennes ne peuvent fournir des éléments de datation, les plus récentes en revanche permettent de situer ces verres à partir du tout début du VI ${ }^{e} s$. Les deux verres de la Bourse retrouvés en stratigraphie sont dans des contextes du $\mathrm{VI}^{\mathrm{e}} \mathrm{s}$. : l'un est du commencement du siècle, l'autre du milieu. A Saint-Blaise 7 fragments sont datables : cinq sont dans la première moitié du $\mathrm{VI}^{\mathrm{e}} \mathrm{s}$., les deux autres dans l'autre moitié. Enfin le fond très mutilé, trouvé au quartier de Besagne à Toulon, est dans une fosse de l'extrême fin du VI ${ }^{e} s$., mais ce dernier verre se différencie des précédents par sa matière verdâtre et moins fine.

Ces symboles chrétiens rappellent bien évidemment le décor des coupes mérovingiennes trouvées dans les nécropoles du nord de la Gaule dans le Namurois (Dasnoy 1956 ; Werner 1956 ; Alenus-Lecerf 1986), l'Aisne (Périn 1972), l'Oise (Legoux 1978, 231-240) et plus exceptionnellement dans les habitats (Docquier 1984) ainsi que dans les cimetières de Westbere, Kent (Jessup 1946, 11-21) et Darent Park, Dartford (Wesbster 1980) en Grande-Bretagne. Les datations avancées s'étagent entre le troisième quart du $V^{e} s$. et le premier quart du $\mathrm{VI}^{e} \mathrm{~s}$., mais les indices chronologiques les plus fiables réduisent cette fourchette de datation entre 475 et 525 . La carte de répartition et l'étude typologique de ces objets ont conduit les archéologues à situer au moins deux ateliers dans les Ardennes et la région de Namur (Périn 1972). D'autres centres existaient probablement, comme le suggère la découverte ancienne à Autun d'un moule en pierre avec décor de chrisme et inscription destiné au soufflage-moulage des coupes en verre (Graillot 1899).

Il n'est pas impossible que les trouvailles provençales soient des productions régionales : leur nombre, la répétition d'un motif, ainsi que l'existence d'ateliers de verriers contemporains de ces coupes sont des indices convaincants pour étayer cette hypothèse.

\section{$\bullet$ Divers}

Pour en finir avec cette énumération, il reste à mentionner la présence d'un fragment de rebord vert bleuté étonnant dans la mesure où il présente une embouchure rectiligne avec l'annonce d'un retour ou d'un bec : peut-être s'agit-il d'une ouverture de section rectangulaire $\left(n^{\circ} 55\right)$.

A signaler aussi un large fond probablement d'une bouteille aux parois verticales ( $n^{\circ} 58$ ), ainsi qu'un fond exceptionnellement coloré en bleu dans la masse et orné de deux anneaux concentriques ( $\left.n^{\circ} 59\right)$. Nous ne savons pas à quelle forme rattacher une anse courte et plate, à peine coudée, et de teinte olivâtre ( $\left.n^{\circ} 57\right)$. Enfin un fragment de verre à vitre a la particularité d'être grugé sur un côté ( $\left.n^{\circ} 56\right)$.

\subsubsection{Un atelier de verrier}

Comme nous l'avons indiqué plus haut, quelques indices nous autorisent à situer dans le quartier actuel de la Z.A.C. Notre-Dame une installation pour le travail du verre. Les témoins de cet artisanat consistent en quelques fragments de creusets vitrifiés et en déchets de verre.

Les trois tessons de céramique : 2 fonds et un rebord, sont toujours vitrifiés à l'intérieur et parfois même sur leur paroi extérieure (fig. $69, \mathrm{n}^{\circ} 62$ à 64). La couche de verre plus épaisse dans les fonds, atteint $3 \mathrm{~mm}$. Formée de cristaux fortement irisés, elle présente un aspect craquelé. A l'origine le verre était de teinte verdâtre. La pâte hétérogène, de teinte orangée, violacée ou rouge brun, contient des grains blancs de calcite et des nodules ferrugineux rouges. 
La composition chimique de cette pâte n'est pas encore connue (9), mais on peut présumer, sans grand risque, qu'elle est réfractaire. Elle a probablement les mêmes propriétés que des autres creusets découverts sur plusieurs sites. Bien que réduits, ces fragments de céramique peuvent également être rattachés à des coupelles de type fort commun dans le répertoire de la vaisselle de terre de la fin de l'Antiquité. Une parenté entre les profils des creusets et celui de quelques céramiques culinaires a été maintes fois observée. Ainsi nous avons pu supposer que les creusets ne sont que des céramiques domestiques réemployées. Cette réutilisation a été prouvée : des analyses faites sur les creusets trouvés à Saint-Jeande-Garguier (B.-du-Rh.) ont établi une concordance entre la matière de ces pots et celles de certaines céramiques communes produites régionalement. Les travaux de laboratoire corroborent l'étude typologique et ne laissent aucun doute sur cette pratique de reconversion d'ustensiles domestiques en instruments pour le verrier (Foy 1990).

Les seconds indices d'un travail du verre consistent en une demi douzaine de déchets ou de ratés de verre. Ces rebuts portent souvent la marque d'un outil (fers ou ciseaux) mais ne nous renseignent guère sur la production. Un seul fragment a été identifié : c'est un pied à double épaisseur, en partie replié $\left(n^{\circ} 61\right)$ : il s'agit probablement d'un pied conique.

Les restes ténus de l'atelier de verrier de Gardanne permettent d'enrichir la liste des officines de l'Antiquité tardive répertoriées en Provence et Languedoc uniquement à partir de creusets et parfois de déchets. Les creusets trouvés sur les sites urbains, périurbains, ruraux ou monastiques, servaient à fondre les matières premières vitrifiables ou plus vraisemblablement un produit semi-fini : la fritte ou des lingots de verre. Il faut sans doute imaginer de petites fabriques dont les productions étaient réservées à une consommation locale ou régionale. Il est malheureusement impossible de situer ici l'atelier en fonction de l'habitat. Cependant il est intéressant de noter la présence, dans le même contexte, de témoins de deux artisanats du feu : le verre et la métallurgie. La concentration de plusieurs activités artisanales est en effet assez banale pour les périodes du Haut Empire ou de la fin de l'Antiquité, en particulier dans les zones suburbaines.

Rien ne nous autorise à assurer que tous les verres trouvés dans le même contexte que les témoins de fabrication proviennent de l'atelier local. Nous sommes pourtant tentés de voir, dans cette association de produits finis et de traces d'artisanat, un indice de plus en faveur de la production régionale de certains verres, en particulier les coupelles à décor chrétien qui, jusqu'à ce jour, ne sont signalées dans les pays méditerranéens qu'en Provence.

\section{Datation de la fosse-dépotoir}

\subsection{LES CERAMIQUES SIGILLEES CLAIRES D ET LES LUISANTES}

La quasi-absence des sigillées claires $\mathrm{D}$ dans la fosse réduit leur valeur chronologique. Le fragment de plat carré peut être ancien : première moitié du $\mathrm{V}^{e}$ s., mais ces pièces luxueuses sont fréquemment conservées. L'unique forme de sigillée orientale phocéenne, elle, est attestée à partir du milieu du $\mathrm{V}^{\mathrm{e}} \mathrm{s}$. Quant au matériel hors fosse, il se rattache à la seconde génération des productions tunisiennes, datée de la dcuxic̀mc moitié du $\mathrm{V}^{\mathrm{e}} \mathrm{s}$. jusqu'au milieu $\mathrm{du} \mathrm{VI}^{\mathrm{e}}$ s. Il ne date que le milieu environnant et non la fosse.

La catégorie des céramiques luisantes qui perdure dans la première moitié du $\mathrm{V}^{e} s$. est absente de la fosse. Les cinq tessons recueillis (fig. 9) proviennent des alentours.

\subsection{LES CERAMIQUES DERIVEES-DES- SIGILLEES PALEOCHRETIENNES}

En Provence, les DS.P. ne peuvent être raisonnablement utilisées comme éléments de datation pertinents, et ce pour des raisons qui tiennent à leur nature propre comme à celle des lieux où elles sont découvertes. Aucun site gaulois de leur époque n'est historiquement daté et rares sont ceux dont l'occupation ait été de courte durée : deux éléments rencontrés par exemple dans les camps du limes où fut datée à l'époque impériale une grande partie des terres sigillées.

Contrairement à celles-ci, des ateliers multiples d'importance variable fabriquèrent des DS.P. de caractéristiques voisines et il est par ce fait plus difficile d'en distinguer les évolutions, chaque lieu de découverte ayant pu être alimenté par des fournisseurs différents établis dans des lieux encore inconnus, et aucun changement technologique ou esthétique ne paraissant avoir accompagné les grands bouleversements historiques. La majorité des DS.P. de la fosse de Gardanne pouvant être attribuée à une production originale, des références précises sont en conséquence absentes. A cela s'ajoutent les incertitudes quant à la durée d'utilisation d'un objet et les pollutions résiduelles pratiquement toujours inévitables, dans la stratigraphie la mieux épluchée comme dans le dépotoir qui nous occupe ici. Seule demeure possible la possibilité d'établir parfois un 
terminus post quem par la présence de monnaies comme ce fut le cas pour Saint-Blaise. Un grand handicap dans l'études des DS.P. est aussi le faible nombre recensé de formes entières, ou tout au moins graphiquement complètes.

Avec la prudence à laquelle incitent ces considérations il n'en est pas moins utile de rechercher, pas à pas, des éléments qui, s'ils n'autorisent pas des datations, permettent de situer dans le temps des périodes d'une durée raisonnable. Ces repères peuvent être soit des objets isolés, soit des ensembles d'objets. Dans ce lot, les renseignements fournis sont contradictoires : le matériel donnant quelques indications se rattachant à des productions soit précoces soit tardives.

Paraissent anciens :

- avec certitude, le bol de forme 6 (fig. 19, $n^{\circ} 110$ ) orangé attribuable à l'atelier de Saint-Julien dont le style de la production est languedocien (Rigoir 1985);

- les deux bords de forme 18 (fig. $20, \mathrm{n}^{\circ} 146$ et 149 ) qui appartiennent à la production classique de l'atelier "marseillais";

- les formes $3 c$ (fig. 18, $n^{\circ} 31$ et 32) dont un exemplaire est signalé à Marseille-Bourse (Cavaillès-Llopis 1986 , fig. $19, n^{\circ} 29$ ), période $1: \pm 400$. Encore faut-il peut-être considérer le profil du marli, plus classique sur cet exemplaire que celui conservé sur la pièce trouvée hors fosse à Gardanne.

Paraissent tardifs :

- les formes 29 (fig. 24) dont les profils abâtardis se retrouvent période $3\left(\mathrm{VI}^{\mathrm{e}} \mathrm{s}\right.$.) à Marseille-Bourse et particulièrement à Saint-Blaise, à la même époque, sur des formes comparables ;

- indiscutablement, le poinçon 2015 , la double rouelle fissurée, imprimée obligatoirement sur une pièce plus tardive que les pièces classiques - signalées dans le répertoire des décors - où elle fut utilisée intacte;

- la croix latine, poinçon 4544, qui n'apparaît pas dans les inscriptions avant la deuxième moitié du $\mathrm{V}^{\mathrm{e}} \mathrm{s}$., postérieurement au chrisme et à la croix monogrammatique (Bayard 1990, 293).

Des comparaisons avec Marseille-Bourse et Saint-Blaise peuvent être établies sur deux ensembles d'éléments : le pourcentage des pièces décorées et celui des vaisselles par rapport aux ustensiles culinaires.

Les stratigraphies établies dans plusieurs sondages à Marseille montrent, au fur et à mesure que l'on avance dans le temps, une raréfaction très nette des décors au poinçon sur les pièces issues de l'officine établie dans - ou proche de - cette cité. A Saint-Blaise, des pourcentages équivalents sont relevés dans les couches tardives. Si l'on tente d'y com- parer le contexte de Gardanne, on constate la même pauvreté décorative : $4,3 \%$, qui peut être attribuée à une dégénérescence similaire.

Le pourcentage de vaisselle, important, semblerait contredire cette datation : il est plus faible, en effet, dans les DS.P. des couches tardives des deux sites de référence. Mais, si l'on prend en compte qu'une part importante (jusqu'à $50 \%$ ) de la vaisselle y est représentée par la Sigillée Claire D et si l'on établit les pourcentages globalement sur les deux catégories, on s'aperçoit que les résultats sont comparables pour les trois sites, assiettes, coupelles et bols de DS.P. venant combler à Gardanne l'absence des formes de Sigillée Claire $\mathrm{D}$, où les ustensiles culinaires se limitent à quelques mortiers. Contrairement au précédent, ce type de comptage n'est donc pas significatif pour des contextes non comparables.

En conséquence, ce lot paraît appartenir à une deuxième génération de cette céramique, ce qui correspond au tournant du $\mathrm{V}^{e}$ au VI ${ }^{e} s$.

\subsection{LA CERAMIQUE COMMUNE GRISE}

L'évolution des objets étant liée à des techniques de fabrication et des habitudes culinaires qui ne se modifient pas de façon importante pendant des dizaines d'années, il ne faut pas rechercher une datation très fine à partir de la céramique commune grise. Nous avons ici affaire à un faciès local ou micro-régional pour la presque totalité des objets, et les comparaisons avec des types analogues découverts sur d'autres sites plus ouverts au commerce, donc plus soumis à d'autres influences ou euxmêmes à l'origine de nouvelles influences, doivent inciter aussi à une certaine prudence.

Les chronologies fournies par la stratigraphie de Saint-Blaise et les comparaisons régionales ne seront pas reprises ici en détail, mais leurs conclusions réutilisées. Les différents types existant à Gardanne sur les objets les plus courants sont connus dans la deuxième moitié du $\mathrm{V}^{\mathrm{e}}$ et au VI ${ }^{\mathrm{e}} \mathrm{s}$. : $\mathrm{A} 1, \mathrm{~A} 2, \mathrm{~A} 2 \mathrm{~A}$, $\mathrm{A} 3, \mathrm{~B} 1, \mathrm{~B} 2, \mathrm{~B} 3, \mathrm{D}, \mathrm{E} \ldots$ Les types $\mathrm{A} 4$ sont déjà bien répandus à Saint-Blaise à partir du deuxième quart du VI ${ }^{e}$ s., mais comme il n'y a pas de séquence stratigraphique immédiatement antérieure, on peut donc en déduire qu'ils sont apparus dans le courant du premier quart du $\mathrm{VI}^{\mathrm{e}} \mathrm{s}$. D'autres formes moins fréquentes apportent aussi quelques précisions. Les amphorettes $\mathrm{F}$ ne sont connues qu'à partir de la fin $\mathrm{du} \mathrm{V}^{e}$ s., les cruches $\mathrm{K}$ à partir du $\mathrm{VI}^{\mathrm{e}}$ s. seulement, comme les formes $O$. Les "tians" ou jattes de forme $C$, que l'on ne rencontre que dans le courant du $\mathrm{V}^{\mathrm{e}}$ s., sont absentes ici. Absents aussi les rebords A6, 
type tardif assez caractéristique de la deuxième moitié ou de la fin du $\mathrm{VI}^{\mathrm{e}} \mathrm{s}$.

Il parait donc logique d'en déduire une datation des premières décennies du VI $s$.

\subsection{LE MOBILIER EN VERRE}

L'examen de l'ensemble des verres de Gardanne nous permet d'avancer une datation pour le comblement de la fosse qui les contenait. Les différents types de verreries recueillis se situent dans une fourchette comprenant le $\mathrm{V}^{e}$ et le $\mathrm{VI}^{\mathrm{e}}$ s. Il est cependant possible d'affiner cette datation en prenant en compte les séries les mieux représentées ainsi que les pièces les plus récentes, à savoir : les verres à pied conique, les objets décorés à l'émail, les fonds dotés d'un symbole chrétien et les supports des verres à tige.

Les pieds coniques sont connus pendant tout le $\mathrm{V}^{e}$ et la dernière partie du VI ${ }^{e} s$. ; le décor d'émail apparaît dès le milieu du $\mathrm{V}^{\mathrm{e}}$ mais connaît son plus grand développement au début du $\mathrm{VI}^{\mathrm{e}} \mathrm{s}$. ; l'ornementation chrétienne sous les fonds est circonscrite entre le dernier quart du $\mathrm{V}^{\mathrm{e}}$ et le premier quart du $\mathrm{VI}^{\mathrm{e}} \mathrm{s}$.; enfin les verres à tige exceptionnels à la fin du $\mathrm{V}^{\mathrm{e}}$, un peu plus répandus dans la première moitié du $\mathrm{VI}^{e}$, sont les verreries les plus communes en Méditerranée à partir du milieu du $\mathrm{VI}^{\mathrm{e}} s$.

Il apparaît ainsi que la période où tous ces verres peuvent coexister ne peut être que la fin $d_{u} V^{e} s$. ou le début du VI ${ }^{e} s$. Deux observations plaident cependant en faveur de l'hypothèse de datation la plus récente : c'est d'abord la présence de deux ou trois spécimens de verre à tige, forme très exceptionnelle avant le $\mathrm{VI}^{\mathrm{e}}$ s., c'est ensuite l'importance numérique des verres ornés d'émail. Cette ornementation prépondérante dans le mobilier recueilli à Gardanne nous suggère ainsi comme datation les deux premières décennies $\mathrm{du} \mathrm{VI}^{e} \mathrm{~s}$.

\subsection{BILAN}

En conclusion, les datations fournies par les matériels issus d'ateliers régionaux de poteries et verreries concordent. Pour une fois, dans un contexte de l'Antiquité tardive, ce ne sont pas les céramiques importées, Sigillées Claires tunisiennes ou orientales, les amphores ou les monnaies qui apportent des critères chronologiques relativement précis.

Les céramiques régionales et les verres moins connus ayant bénéficié d'études récentes conduites avec une méthodologie rigoureuse apparaissent à présent d'une utilité comparable. Mais ce type de démarche n'a pu se concevoir que dans le cadre d'une recherche en équipe, soumise constamment au contrôle de l'échange des éléments d'analyse et à la confrontation des résultats.

La richesse de ces matériels variés résulte d'une activité artisanale importante. Sur place, l'activité des bronziers et des verriers est attestée par les moules et les creusets.

Une partie des DS.P. est de production marseillaise, celles d'Apt ont été évoquées à plusieurs reprises, mais une proportion notable de vaisselles paléochrétiennes ne ressemble pas à ce que l'on trouve à Marseille, à Saint-Blaise, à Aix, à Saint-Jean-deGarguier ou à Apt. On peut en conséquence envisager une production locale ou régionale proche. Cette quasi-autarcie semble confirmée par l'absence de Sigillées Claires D importées. De plus, à l'époque qui nous intéresse ici, l'atelier de Marseille ne produisait que de rares formes de vaisselle. Ainsi les carences d'approvisionnement extérieur apparaissent compensées par une fabrication dont l'importance est anormale à cette époque et qui ne peut être que locale.

La commune grise présente des caractères qui plaident en faveur d'une fabrication relativement proche. Elle reprend des éléments de formes bien répandus dans la région, mais avec un faciès que l'on

\begin{tabular}{|c|c|c|}
\hline Espèces & NRD & NMI \\
\hline Bœuf (Bos taurus) & 219 & 6 \\
\hline Caprinés (Ovis aries et Capra hircus) & 1037 & 32 \\
\hline Porc (Sus scrofa domesticus) & 281 & 10 \\
\hline Cerf (Cervus elaphus) & 35 & 2 \\
\hline Lièvre (Lepus europaeus) & 2 & 2 \\
\hline Lapin (Oryctolagus cuniculus) & 11 & 3 \\
\hline Coq (Gallus domesticus) & 14 & 5 \\
\hline Oie (Anser domesticus) & 2 & 1 \\
\hline Pigeon ramier (Colomba palumbus) & 1 & 1 \\
\hline Perdrix rouge (Alectoris rufa) & 3 & 1 \\
\hline Grive musicienne (Turdus philomelos) & 1 & 1 \\
\hline Oiseaux indéterminés (Aves sp.) & 4 & \\
\hline Poissons & 2 & \\
\hline Cheval (Equus caballus) & 29 & 4 \\
\hline Ane (Aquus asinus) & 3 & 1 \\
\hline Chien (Canis familiaris) & 65 & 3 \\
\hline Amphibiens & 1 & \\
\hline Sanglier (Sus scrofa scrofa) & $1 *$ & \\
\hline Homme (Homo sapiens sapiens) & 30 & 1 \\
\hline Nombre de restes déterminés & \multicolumn{2}{|c|}{1741} \\
\hline Nombre de restes indéterminés & \multicolumn{2}{|c|}{531} \\
\hline Nombre des restes total & \multicolumn{2}{|c|}{2272} \\
\hline
\end{tabular}

* défense avec poli d'usage

Tabl. X Espèces animales représentées (nombre de restes déterminés et nombre minimum d'individus). 
peut qualifier de local. La comparaison avec le matériel de Saint-Jean-de-Garguier, autre site rural de l'arrière-pays marseillais, distant d'une vingtaine de kilomètres, met en évidence d'autres exemples de variations de détail des types principaux. Ces variations, différentes dans chacun des deux sites, se manifestent notamment dans la forme des rebords à la mode régionale qui revêtent des caractères particuliers.

On ne sait rien de précis sur l'habitat qui a utilisé le matériel qui nous est parvenu dans cette fosse. Il devait être relativement important pour concentrer, parallèlement à une activité domestique représentée par la céramique et les déchets culinaires, des ateliers consacrés aux arts du feu qui ne se limitaient pas à la fabrication d'ustensiles d'usage courant comme cela parât être le cas pour la forge. Les éléments mobiliers issus des moules de bronzier témoignent d'un travail bien particulier pour une clientèle soucieuse d'un certain luxe, lequel se manifeste aussi par la qualité des décors émaillés et moulés des verreries.

\section{Les déchets culinaires : ossements et coquillages}

\subsection{LA FAUNE TERRESTRE}

La fouille partielle de la fosse de Gardanne a livré 2272 restes fauniques (à l'exclusion des restes de mollusques et d'un objet manufacturé en os). C'est un échantillon modeste, qui a malgré tout permis la détermination de 17 espèces animales, homme non compris (tabl. $\mathrm{X}$ ).

Le régime alimentaire assez varié des habitants et l'utilisation mixte de la fosse expliquent la relative diversité des espèces présentes et l'état de conservation contrasté des vestiges osseux. Des restes très endommagés, le plus souvent réduits à l'état d'éclats et d'esquilles, se mêlent à des parties squelettiques bien conservées, parfois intactes, présentant des connexions anatomiques. Il ne s'agit donc pas à proprement parler d'une fosse à déchets domestiques spécialisée, mais plutôt d'une fosse de décharge à utilisations multiples, recueillant aussi bien des résidus de cuisine que des cadavres d'animaux domestiques non consommés, des chutes de débitage de bois de cerf ou des résidus d'un artisanat de bronziers.

Le mauvais état de conservation des restes alimentaires - attesté par l'état de fragmentation de la presque totalité des métapodes de caprinés, qui d'ordinaire résistent assez bien à l'érosion, par les traces du travail intense des chiens sur ces déchets, largement responsables de leur destructionindique sans ambiguïté que ces restes de table n'ont pas été jetés rapidement dans la fosse après consommation, à l'exception de certains d'entre eux, mais qu'ils y ont été rassemblés après avoir séjourné quelque temps ailleurs. Ils proviennent sans doute en grande part de balayages et de curages de zones de déchets plus proches des habitations. Il y a donc fort peu de chance de se trouver ici en présence des restes de quelques repas, des artisans bronziers par exemple. L'échantillon exprime plus probablement le régime alimentaire des occupants de cet habitat sur une séquence de quelques mois.

Ceci invite à considérer avec prudence l'éventail des espèces consommées, qui peut simplement témoigner de la contribution modeste de gibier à l'alimentation sur une assez longue période, et non pas révéler l'opulence de quelques copieux repas.

Sur les 2272 restes recueillis, 1741 restes ont pu être déterminés, dont 1605 proviennent d'espèces consommées, domestiques ou sauvages.

\section{$\square$ La chasse et la pêche}

Comme il est habituel dans les contextes gallo-romains, la part des produits sauvages dans le régime alimentaire est réduite : elle est représentée ici par 3,4\% des restes osseux, ce qui indique une présence certes faible mais sans doute constante de l'appoint fourni par la chasse et la pêche. On y remarque du lièvre mais surtout du lapin, inconnu à cette période dans le reste de la Gaule, mais dont la présence ne surprend pas sur le pourtour méditerranéen. Si le faible nombre de restes autorise ce rapprochement, on peut relever que la viande de lagomorphe paraît consommée aussi fréquemment que celle de poule (équivalence tant en nombre de restes qu'en nombre d'individus).

A côté de ce petit gibier, le cerf est représenté par au moins 2 individus et 28 restes (compte non tenu des restes de bois). Plusieurs éléments en connexion anatomique indiquent que des parties non consommables de l'animal ont été jetées dans la fosse aussitôt après sa découpe. A la différence des caprinés, le cerf semble être traité rapidement, sans doute à la faveur de l'événement un peu exceptionnel que représentc la préparation d'une grosse pièce de gibier. Ainsi retrouve-t-on intacts les éléments de l'articulation du membre antérieur, portant la trace franche du coup de couperet qui l'a isolée, ou les bas de pattes jetés entiers dans la fosse (métapodes, épiphyses distales et phalanges en connexion).

Les oiseaux sauvages n'apparaissent qu'en très petit nombre : il s'agit d'un pigeon ramier, d'une perdrix rouge et d'une grive musicienne. Il faut ici tenir compte de la fragmentation intense de la faune, préjudiciable aux ossements d'oiseaux, et d'une maille de tamisage qui n'a peut-être pas permis de conserver toute la microfaune (absence de rongeurs et d'insectivores par exemple).

Pour les mêmes raisons, le chiffre de 2 restes de poissons ne permet pas de conclure à un apport négligeable de la pêche dans l'alimentation, d'autant que le nombre important de restes de mollusques marins (huîtres en particulier) atteste le recours aux ressources de la mer, distante de $20 \mathrm{~km}$ de Gardanne. 

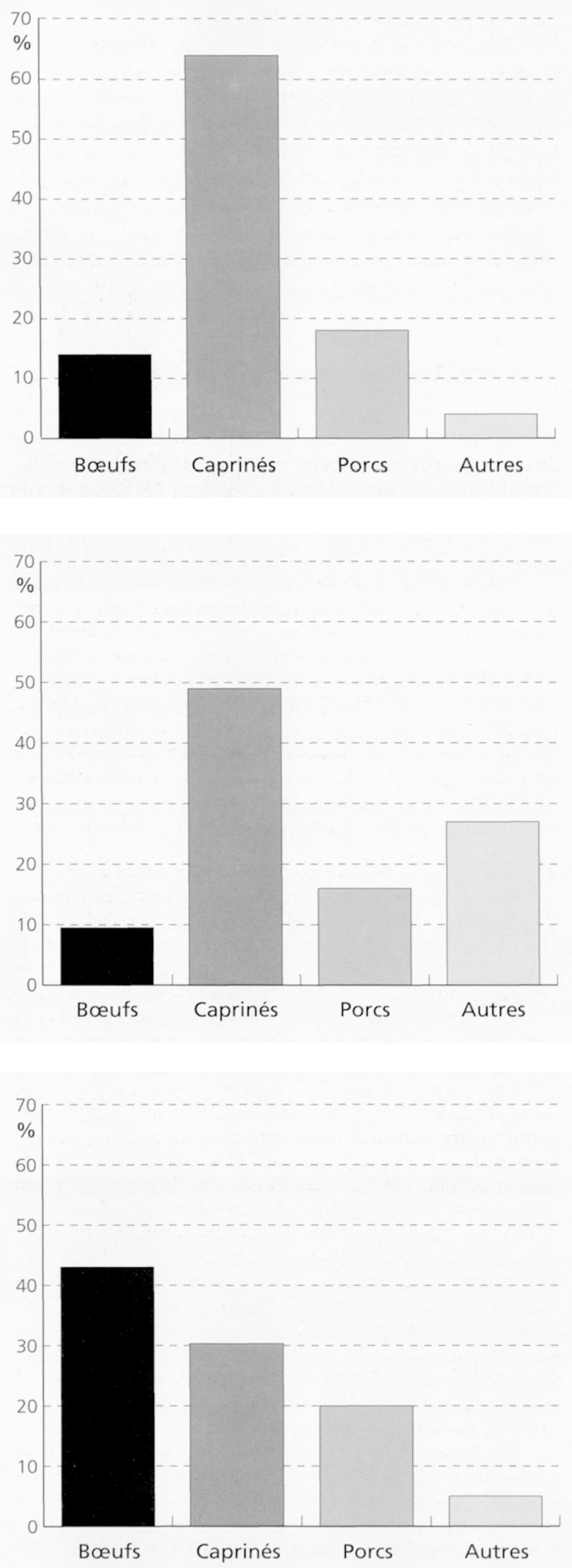

\section{$\square$ L'importance des caprinés}

Bœuf, caprinés et porc constituient bien entendu la base du régime alimentaire carné, formant $95 \%$ du nombre de restes des espèces consommées.

Pour l'époque antique, les témoins osseux disent assez clairement que bœuf et porc se disputent la première place dans les régimes alimentaires - en nombre d'individus consommés et non pas en poids de viande. Le bøuf domine le plus souvent sur les sites militaires et urbains, le porc est plus présent sur les sites ruraux ou pré-urbains.

En France, les sites antiques du Midi méditerranéen font exception à cette règle, et ce sont les caprinés qui y occupent souvent la première place en nombre d'individus. C'est ainsi le cas, pour les Bouches-du-Rhône, à Marseille-Bourse (II'$V^{e}$ s.) (Jourdan 1976), à Calissanne II (III s.) (Poulain 1964), pour l'Aveyron, à La Graufesenque (I er-II $^{\mathrm{e}}$ s.) (Ibid.), pour le Gard, à La Condamine (IVe s.) (Ibid.). En revanche, la faune du site d'Ambrussum dans le Gard ( ${ }^{\text {er }}$ s. av. J.-C.- $\mathrm{II}^{\mathrm{e}}$ s. ap. J.-C.) (Columeau 1989) montre une prédominance classique des suidés. Une telle suprématie des caprinés peut se rencontrer, mais très rarement, en d'autres régions, comme sur le vicus de Lewarde dans le Nord par exemple ( $\left(\mathrm{I}^{\text {er }}-\mathrm{II}^{\mathrm{e}} \mathrm{s}\right.$.) (Vadet 1979), mais elle est surtout caractéristique des terres pauvres propices à l'élevage ovin.

La faune de Gardanne confirme cette place particulière prise par l'élevage des caprinés dans l'économie du Midi méditerranéen : moutons (et chèvres) y dominent largement avec $67,5 \%$ des restes, suivis par le porc $(18,3 \%)$ et par le bœuf $(14,2 \%)$ (fig. 71$)$. On retrouve cette même hiérarchie en nombre minimum d'individus, les caprinés représentant $66,6 \%$ des 48 bovins, caprinés et porcs dénombrés dans la fosse, les porcs $20,8 \%$ et les bovins $12,5 \%$ (fig. 72 ). En termes de poids de viande consommés (10), les résultats diffèrent : les caprinés n'occupent plus que la seconde place de la trilogie, apportant un tiers des ressources alimentaires carnées (fig. 73). L'importance de la viande de mouton et de chèvre dans le régime témoigne bien d'un système alimentaire soumis aux dispositions pastorales de la région et différant ainsi des coutumes de table ordinaires du monde romanisé.

Elevés avant tout pour leur laine - ce dont témoignent les agronomes de l'Antiquité -, les ovins sont en règle générale abattus assez tardivement sur les sites antiques. C'est le cas à Gardanne où la majorité des bêtes est abattue après 2 ans : une partie entre 2 et 3 ans, une autre, plus importante, vers 3-4 ans. La présence ici de quatre jeunes d'environ 3 mois (pour 32 individus) fait contraste avec leur rareté sur un site urbain comme celui de La Bourse, où l'on ne relève que trois jeunes de 3 mois pour 193 individus (Jourdan 1976). Ils témoignent très probablement ici d'une consommation directe des produits de l'exploitation agricole, fournissant un registre d'animaux différent de celui rencontré dans les milieux urbains. L'abattage de ces jeunes ne trahit pas forcément un luxe de table, mais peut résulter de sacrifices nécessaires à pratiquer au sein du troupeau. En revanche, la

171 Pourcentages des espèces consommées (en nombre de restes déterminés).

172 Pourcentages des espèces consommées (en nombre minimum d'individus).

- 73 Pourcentages des poids de viande consommés. 
consommation de quelques animaux abattus vers $12-18$ mois peut évoquer un recours épisodique à une viande de boucherie de qualité.

Le nombre de chèvres est difficilement quantifiable au sein de ce matériel fortement esquillé, mais leur présence est aisément repérable - et minoritaire - parmi les chevilles osseuses (8 éléments de chevilles de chèvres pour 42 éléments de chevilles de mouton, essentiellement des mâles).

\section{$\square$ Les porcs}

En nombre minimum d'individus, on abat à Gardanne plus de porcs que de bovins, fait plus caractéristique des sites ruraux que des sites militaires et urbains. Mais le porc y est, en termes de poids de viande, l'espèce la moins consommée. Il ne représente en effet que $21,5 \%$ de l'apport en viande, soit deux fois moins que le bœuf et un tiers de moins que les caprinés.

Selon le cas général rencontré sur les sites antiques, les porcs sont abattus le plus souvent avant 2 ans, âge où ils atteignent un état optimal d'engraissement qui les rend les mieux propres à la consommation. Quelques individus, sans doute conservés comme reproducteurs, ont été abattus plus tard, vers 3 ans et demi. Deux porcelets et un jeune de moins de 1 an apportent en outre à la table des viandes plus fines. De la même façon que pour les jeunes caprinés, leur présence ne signale pas forcément un choix gastronomique mais peut résulter d'une nécessité.

\section{$\square$ Les bovins}

Les bovins fournissent presque la moitié de la viande consommée à Gardanne (46,5\%). Cet apport est très minoritaire en comparaison des moyennes observées sur d'autres sites. Le contexte pastoral particulier au pourtour méditerranéen et le caractère rural du site ont en effet limité l'importance que prend ordinairement la viande de bœuf à la table gallo-romaine.

Selon l'analyse synthétique réalisée par R.-M. Luff (1982), les bovins sont abattus après 3 ans et plus sur la majorité des sites de l'empire romain. A Gardanne, deux individus ont été consommés entre 3 ans et demi et 4 ans, et deux autres au-delà. Deux animaux ont cependant été abattus à moins de 2 ans- 2 ans et demi. Mais on ne remarque aucun reste de très jeune veau, ce qui n'a rien pour étonner : sa consommation n'entre pas dans les habitudes avant les temps modernes, et sa présence est aléatoire sur les sites antiques comme médiévaux.

Cet abattage assez diversifié, constaté aussi bien pour les caprinés que pour les porcs ou les bovins, fournissant une majorité d'animaux adultes mais aussi un contingent non négligeable de bêtes immatures ou subadultes, parle en faveur d'une consommation autarcique basée sur l'élevage pratiqué sur place plutôt que d'un approvisionnement extérieur.

\section{$\square$ La volaille}

L'apport de la volaille paraît assez limité, coqs et oies n'ayant livré que 16 restes osseux. Ils témoignent de cette prédominance habituelle de la consommation de coq sur celle d'oie. Là encore, il est possible que la sévère érosion subie par le matériel osseux ait joué en la défaveur des restes d'oiseaux domestiques, et qu'il faille surévaluer la participation des poules et des poulets au régime alimentaire.
L'ensemble de ces éléments dessine une table où l'on ne consomme pas les rebuts trop âgés des troupeaux, ni non plus des bêtes de boucherie abattues systématiquement avant leur maturité. Néanmoins, la présence de viande de très jeunes ou de jeunes animaux y semble plus qu'épisodique. Le régime trouve en outre une certaine variété dans l'appoint, marginal mais non exceptionnel, de viandes de petit ou gros gibier. C'est semble-t-il une table à caractère rural, qui paraît assez aisée, probablement sans luxe, mais dont les viandes de qualité ne sont pas exclues.

\section{$\checkmark$ La taille des animaux d'élevage}

Même en faible nombre, les restes animaux de la fosse de Gardanne témoignent de cette situation pastorale bien particulière que l'on connaît à l'époque romaine dans les provinces de l'Empire : la juxtaposition des troupeaux indigènes dc pctitc staturc ct des bctcs romaincs de plus haute taille, probablement importées (Audoin-Rouzeau 1991). Au sein de ce mouvement d'importation, le cheval semble avoir eu la priorité, suivi du bœuf, puis du mouton (Audoin-Rouzeau à paraître).

A Gardanne, la présence de mouton de grande taille n'est pas décelable : les quelques estimations de taille (11) qui ont pu être effectuées (sur 2 métapodes et 2 calcanéums) indiquent des animaux de $57,3 \mathrm{~cm}$ de hauteur au garrot moyenne (var. 52,5/63,5 cm), c'est-à-dire d'un petit gabarit conforme à celui des ovins indigènes (voire même légèrement inférieur, mais les données sont ici trop rares pour l'affirmer). Il est probable que la consommation importante qui est faite de viande de capriné à Gardanne se fonde en majeure partie sur les produits de l'élevage local traditionnel.

Le jeune âge des suidés et la fragmentation de leurs ossements n'ont pas permis d'estimer leur gabarit.

En revanche, les mensurations des restes de bovins témoignent de l'existence de deux populations bien contrastées (12) : une diaphyse de métatarsien révèle un individu de moins de $90 \mathrm{~cm}$ de hauteur au garrot. A côté de cet animal de très petit format, figurent un veau qui atteint $136,2 \mathrm{~cm}$ avant même sa maturité (bas des pattes postérieures en connexion) - et qui se range ainsi parmi les plus grands animaux romains - et un autre individu de $126,2 \mathrm{~cm}$ de hauteur au garrot, soit un animal "romain" de taille moyenne.

La même juxtaposition de deux types d'animaux se constate chez les chevaux. Les restes très incomplets d'au moins quatre individus (trois adultes et un poulain) ont été évacués dans la fosse, ne portant, bien sûr, aucune trace de consommation. Pour un certain nombre de pièces (tarsiens, métacarpiens et dents jugales) s'est posé le problème de leur attribution au cheval ou au mulet, ou encore à l'âne. Ces ambiguïtés ont pu être levées grâce à la contribution de V. Eisenmann. Deux chevaux atteignent respectivement d'après leurs métacarpiens les tailles au garrot (13) de $143,6 \mathrm{~cm}$ et $149,4 \mathrm{~cm}$, s'inscrivant ainsi dans la variation normale des chevaux romains. En revanche, un radius témoigne d'un individu de $127,2 \mathrm{~cm}$ au garrot, une taille rapportable à celle des chevaux de l'Age du fer, ayant coexisté avec les races romaines après la Conquête.

L'âne est représenté par deux tarsiens et par un humérus dont le petit format et l'aspect gracile permettent de l'attribuer à cette espèce avec une quasi-certitude. Endommagé dans sa partie proximale, il ne permet pas une estimation précise de la taille de l'animal, qui ne saurait cependant avoir 
dépassé quelque $113 \mathrm{~cm}$ au garrot. Cette nouvelle identification de l'âne en contexte gallo-romain vient compléter les informations, encore peu nombreuses, connues sur son expansion : espèce introduite dans l'Empire par les Romains, on en trouve semble-t-il les premières traces en France au $I^{\text {er }} s$. av. J.-C., mais de manière plus certaine à partir du $\mathrm{I}^{\text {er }} \mathrm{s}$. ap. J.-C. Dans l'ensemble de l'Europe à l'époque antique, ses découvertes restent rares, même s'il est probable que la difficulté posée par sa différenciation d'avec les chevaux de petite taille, réduit les chances de l'identifier et minimise peut-être sa présence réelle.

En France, il est entre autres reconnu sur les sites antiques de Cazères (Poulain 1966), de Saint-Marcel (Ibid.), de Valflaunès (Poulain 1972), d'Ambrussum (Columeau 1989), de Levroux (Derville, Poplin 1977) et de Mariana en Corse (Vigne 1989). Hors de France, l'âne est également rare, mais attesté par exemple sur les sites romains de Tac-Fövenypuszta en Hongrie (Bökönyi 1974)), et de Bad-Kreuznach (Johansson 1987), Abusina-Eining (Lipper 1981-82) et Kempten en Allemagne (Boessneck 1957).

\section{$\square$ Les chiens}

Les trois individus dont on retrouve 65 restes dans la fosse appartiennent à des animaux adultes. Leurs squelettes sont loin d'être complets, mais de nombreuses connexions anatomiques et le très bon état de conservation attestent qu'ils y ont été enfouis assez rapidement après leur mort, même si quelques traces de crocs montrent que des chiens ont eu le temps d'y porter la dent. On ne trouve pas dans cette fosse de ces très petits chiens "de compagnie", caractéristiques des sélections opérées à la période romaine. Il s'agit ici de trois animaux de gabarit moyen, d'une hauteur au garrot de 50 à $55 \mathrm{~cm}$ (14)

\section{$\square$ Les animaux intrusifs}

La fosse n'a livré aucun reste d'insectivore ou de rongeur parasite des habitations humaines. Le seul élément de microfaune à signaler - à l'exception des poissons et des oiseaux - consiste en un reste d'amphibien.

\section{$\square$ Manufacturation}

Le travail du bois de cerf se signale par la présence de cinq pointes d'andouiller dont l'une porte une trace de sciage, et d'une base de bois de chute sciée à quelques centimètres au-dessus de la perlure, attestant l'activité de récupération de cette matière première.

Les métacarpiens et radius des équidés, qui font souvent l'objet d'une récupération privilégiée, se retrouvent intacts dans la fosse. Mais le travail sur os est attesté par une petite chute de débitage, présentant une trace de sciage.

Il faut également signaler un objet façonné en os, en forme de cabochon, ainsi qu'une défense de sanglier fendue longitudinalement et dont toute la face de fracture a été polie par un frottement répété, comme si la pièce avait servi de “lissoir”.

Les 1741 restes osseux déterminés de la fosse de Gardanne représentent un petit ensemble. Mais, outre les principaux aspects du régime alimentaire qu'ils permettent de restituer, ils témoignent bien, à leur échelle, des traits ambivalents de l'économie de l'Antiquité tardive dans laquelle ils s'inscrivent : influence romaine dans l'importation et l'élevage de bœufs et de chevaux de grande taille, ou dans l'intro- duction de l'âne sur l'exploitation agricole, et persistance des habitudes et des contraintes locales dans l'utilisation des petits bovins et chevaux locaux, comme dans le recours aux caprinés pour fonder le tiers des ressources alimentaires carnées (15).

\subsection{LES COQUILLAGES MARINS}

La fosse a livré une certaine quantité de coquillages marins : 174 exemplaires, nombre minimum d'individus (NMI), composés de 211 coquilles et 54 fragments. Ce nombre ne représente qu'une infime partie du matériel conchyliologique réellement stocké dans cette fosse. En effet, seuls les coquillages récupérés par la Société Archéologique Méditerranéenne, à partir de critères probablement visuels : quelques gros fragments, ont été étudiés ici. Il convient de garder ce fait à l'esprit et de ne voir dans ces lignes, qu'une étude ponctuelle sur un échantillonnage des coquillages marins.

\section{$\square$ Biotope}

Tous ces coquillages marins ont été ramenés volontairement sur le site de Notre-Dame. Pour se faire une idée du lieu de leur cueillette, il convient d'étudier le biotope dans lequel vivent actuellement les mêmes espèces, à l'état naturel.

\section{- Biotope des coquillages les plus nombreux}

Les espèces dominantes sont les huîtres : ostrea lamellosa Brocchi 1814 et sa variante cyrnusi Payr. 1826 qui représentent $62,6 \%$ de l'ensemble, et les peignes (autres noms vernaculaires : vanneaux, pétoncles...) : proteopecten glaber L. 1766 , soit 33 \%. Les huîtres vivent en grappes dans les eaux marines à de faibles profondeurs (étage médiolittoral) près des estuaires des fleuves côtiers. Une seule valve portait des vermets : ce chiffre est trop faible pour permettre d'affiner l'image du biotope des huîtres. Les peignes vivent librement sur les fonds sableux et détritiques depuis la surface jusqu'à $200 \mathrm{~m}$ (étages infralittoral et circalittoral). Ces deux espèces ont grandi sur des fonds ne contenant pas une énorme masse de fragments de coquilles-épaves. En effet, aucun fragment de coquillage mort ayant été amalgamé à la valve d'un coquillage vivant n'a été retrouvé. Les huîtres ont vécu accrochées à des rochers.

\section{- Biotope des autres espèces faiblement représentées}

Les autres espèces aux quantités anecdotiques ( 1 ou 2 exemplaires) proviennent de faibles profondeurs ; c'est la biocénose de la roche à algues photophiles : des rochers, recouverts d'algues et pouvant être découverts quelques heures, servent de point d'ancrage à la patella caerulea L. 1766, les fonds vaseux sont parcourus par le murex trunculus L. 1758 (nom vernaculaire : rocher), les spondylus gaederopus L. 1766 (huîtres à charnière) vivent accrochés à des rochers ou à des pieux immergés en permanence. Les sables bien calibrés ou les vases abritent la tapes decussata L. 1758 ou pullastra Montagu 1803 (palourde ou clovisse). Seul le triton nodiferum Lamarck 1822 ou conque a un biotope différent : il vit au large des côtes rocheuses mais on peut trouver des exemplaires plus ou moins fragmentés, rejetés sur le rivage après les tempêtes. 


\section{- Conclusions}

Le biotope cerné par l'examen de ces coquillages correspond mieux à des étangs ou une côte sableuse qu'à une côte rocheuse. L'étang de Vaïne, partie orientale de l'étang de Berre, correspond assez bien à ce biotope ; il est situé à vol d'oiseau à $16 \mathrm{~km}$ de Gardanne, avec le lieu-dit moderne des Cadestaux (commune de Vitrolles) et ses petits fleuves côtiers. La mer libre la plus proche est rocheuse : l'Estaque et ses collines protégeant la baie située à vol d'oiseau à $20 \mathrm{~km}$ de Gardanne. Seuls les trois fragments de triton nodiferum Lamarck 1822 pourraient à la rigueur en provenir.

Les coquillages ont été ramassés, soit à pied, soit en plongée en apnée légère, dans des eaux peu profondes, et sans outillage particulier. En effet, les bancs naturels d'huitres sont peu profonds et le peigne peut être ramassé, quand il est abondant, en piétinant le sable comme cela se pratique encore sur certaines côtes de France.

\section{$\square$ But alimentaire}

\section{- Intérêt diététique}

La quasi-totalité $(95,4 \%)$ des coquillages recueillis dans cette fosse appartient à deux espèces comestibles d'excellente qualité : l'huître et le peigne dont l'aspect et le goût rappellent celui de la coquille Saint-Jacques. Ces coquillages étaient réputés chez les Grecs et les Romains et consommés dès la Protohistoire dans la région de l'étang de Berre (Brien 1988).

Outre leur intérêt gustatif certain, ces coquillages ont une richesse, intéressante pour la nutrition, en éléments minéraux (oligo-éléments) et même en vitamines (dont une quantité importante de vitamine $C$ pour l'huître par exemple), en suspension ou en solution dans les $80 \%$ d'eau, ainsi que des protéines (10 à $15 \%$ pour $100 \mathrm{~g}$ de chair), des lipides et des sucres. Une étude précédente avait démontré que deux douzaines d'huîtres égalaient $100 \mathrm{~g}$ de porc ou $200 \mathrm{~g}$ de poisson du point de vue calorique mais apportaient davantage de vitamines et d'oligo-éléments (Brisou 1985, 110). L'apport des coquillages marins pouvait compenser de notables déficits dans l'alimentation des populations.

\section{- Taille du coquillage}

Ces coquillages ont été ramassés après avoir été choisis en fonction de leur taille. Celle-ci est dans la normale ou même assez élevée pour l'espèce. La valve supérieure de l'ostrea lamellosa Brocchi 1814 a une moyenne de 6 à $7 \mathrm{~cm}$ de haut pour une largeur de 5,7 à $6,8 \mathrm{~cm}$; la moyenne de l'ostrea cyrnusi Payr. 1826 se situe à $9 \mathrm{~cm}$ de haut et $5,7 \mathrm{~cm}$ de large avec un maximum de $11,5 \mathrm{~cm}$ de haut, $7 \mathrm{~cm}$ de large et un poids actuel de $90 \mathrm{~g}$ pour une seule valve supérieure. Les proteopecten glaber L. 1766 sont, eux aussi dans la moyenne de leur espèce : $6 \mathrm{~cm}$ de haut pour $5,9 \mathrm{~cm}$ de large.

Ces chiffres indiquent un ramassage à l'état adulte du coquillage pour en espérer le maximum de chair. Ils ne suggèrent en aucun cas une surconsommation du coquillage quelle que soit sa taille, jeune ou adulte, entrainant à plus ou moins brève échéance l'épuisement des bancs naturels. Le poids de cette cueillette effectuée par l'homme n'est pas un facteur d'éventuelle destruction des espèces naturelles.

\section{$\square$ Techniques d'ouverture}

\section{- Le peigne}

I.es traces d'ouverture des coquillages sont les plus nettes dans le cas des proteopecten glaber L. 1766. Sur un total de 67 valves, seules 4 ne présentaient aucune trace visible, soit moins de $6 \%$. Toutes les autres valves ont été ouvertes en sectionnant un coin de l'oreillette, ce qui permettait de faire passer une lame plate à l'intérieur afin de couper le muscle adducteur qui retient serrées les deux valves. La coupure est réalisée en biais sur une petite dimension, avec un instrument tranchant.

Cet emploi si régulier du couteau, rappelle les sites languedociens de la même période : Lunel-Viel et Loupian (Brien 1990 et à paraître). La cassure de la totalité de l'oreillette antérieure de la valve droite, technique de la Protohistoire, est nettement moins fréquente (Brien 1988). Ces coquillages ont donc été consommés crus puisqu'il a été nécessaire de couper le muscle adducteur de l'animal encore vivant.

\section{- L'buître}

Le cas de l'huître est moins net. On ne remarque pas de traces de pointes de couteau sur le bord ventral comme à Loupian et sur les sites de l'étang de Thau (Brien à paraître). Quelques coquilles ont subi une élévation de température entraînant une carbonatation de la valve, indice d'une ouverture à chaud d'un coquillage consommé cru ou cuit suivant le temps d'exposition à la chaleur. Quelques coquilles ont un aspect cendreux ou même brûlé : 3 valves d'huitres brûlées et 1 cendrée (pour le peigne : 2 cendrées et 1 brûlée). Plutôt que d'une technique culinaire, il s'agirait de coquillages jetés dans le feu ou dans la cendre encore chaude puis stockés avec les valves ouvertes en force, avec le reste des déchets dans le dépotoir.

\section{$\square$ Culture des coquillages?}

Parce qu'il vit librement sur les fonds, le peigne se prête très mal à la culture, de nos jours, malgré la sophistication des méthodes employées. On peut considérer qu'il n'y avait aucune culture de ce coquillage dans l'Antiquité tardive. Les huîtres ne présentent pas de traces nombreuses d'agglutination autour d'une cavité : pieu, fascine ou même tuile immergés. Cinq valves inférieures avaient l'empreinte d'une cavité, ce qui est fort peu : 8,47\%. Rares aussi sont les blocs d'huîtres : seules deux huîtres avaient grandi soudées à une autre valve $(3,38 \%)$. On peut donc affirmer que ces coquillages proviennent de bancs naturels prospectés à pied ou en plongée légère.

\section{$\square$ Transport et commerce}

La présence des coquillages à Gardanne soulève diverses questions. Qui a ramassé ces coquillages : des femmes et des enfants comme encore au début du $\mathrm{XX}^{\mathrm{e}} \mathrm{s}$. sur les côtes françaises ? Ces ramasseurs, riverains des étangs, vendaient-ils eux-même directement aux populations avoisinantes ou bien à des commerçants qui en assuraient le transport puis la redistribution au détail ?

Ces coquillages ont été acheminés sans techniques de conservation (stockage d'une seule valve dans une amphore par exemple), les bivalves ont été transportés avec leurs deux valves car le nombre des valves droites et gauches est sensiblement le même pour les huîtres et les peignes. Cela devait entraîner un poids élevé des marchandises transportées. En effet, si l'ostrea lamellosa Brocchi 1814 est relativement légère, les ostrea cyrnusi Payr. 1826 sont assez lourdes, avec un poids actuel à sec de $90 \mathrm{~g}$ pour une seule valve. La plus grosse des deux valves inférieures du spondylus gaederopus L. 1766 pèse à elle seule et actuellement, $140 \mathrm{~g}$. Comme la source d'approvisionnement est à plus de $16 \mathrm{~km}$ à vol d'oiseau, des coquillages pesants ont été régulièrement transportés dans des vanneries (matériau qui s'altère le moins avec le sel), assez 
rapidement pour éviter une altération dangereuse pour la santé du consommateur sur des routes reliant Gardanne à la mer. C'est un exemple d'exploitation à peu de frais, sans grande technique ni connaissances de la faune (coquillages) et de la flore (joncs) du bord de mer, et pouvant être réalisée pcndant toutc l'annéc car les tempêtes sont rares sur l'étang de Berre.

\section{$\square$ Comparaisons}

Des sites de la même région et de la même période chronologique ont livré beaucoup de matériel conchyliologique : Saint-Blaise et sa masse énorme de coquillages marins (Brien à paraître), et quelques sites encore en cours de fouilles dans la zone de l'étang de Berre. A Saint-Blaise, le choix est plus large avec des huîtres, des peignes, des coques et des moules, mais l'huître représente, à elle seule, la moitié des coquillages consommés. La Pousaraque de Gignac a livré exclusivement des huîtres et des peignes alors que ces coquillages ont vécu dans un biotope où croissaient d'autres espèces (fragments de coquilles-épaves sur les coquillages consommés). Les deux seules espèces : huîtres et peignes, ont été retrouvées sur le site des Clapières à Saint-Mitre-les-Remparts et au Jardin du Théâtre de Verdure de Vitrolles. La plus grande variété dans les espèces recueillies à Saint-Blaise pourrait s'expliquer par la proximité immédiate des étangs, les autres sites ne sont pas directement riverains du rivage. Elle pourrait être due aussi au fait que Saint-Blaise a été une agglomération importante (où vivaient les ramasseurs de coquillages ?), alors que les autres sites ne sont que des exploitations agricoles, des villae abritant une population moindre et ayant d'autres activités. La proximité du rivage et la nature du site seraient des paramètres importants dans la sélection des coquillages, entraînant une alimentation d'origine marine différente.

\begin{tabular}{|l|c|c|}
\hline \multicolumn{1}{|c|}{ Espèces } & NMI & $\%$ \\
\hline Mactra glauca Born 1778 & 1 & 0,57 \\
Murex truncullus L. 1758 & 2 & 1,14 \\
Ostrea lamellosa Brocchi 1814 & 109 & 62,64 \\
Proteopecten glaber L. 1766 & 57 & 32,75 \\
Spondylus gaederopus L. 1766 & 2 & 1,14 \\
Tapes decussata L. 1758 & 1 & 0,57 \\
ou pullastra Mont. 1803 & & \\
Triton nodiferum Lamarck 1822 & 1 & 0,57 \\
Patella caerulea L. 1766 & 1 & 0,57 \\
\hline
\end{tabular}

Une comparaison avec les sites du Languedoc peut être établie mais avec prudence car les destins de la Provence et du Languedoc sont différents dans l'Antiquité tardive. Un site urbain, Lunel-Viel, a livré de nombreuses espèces même si le pourcentage des coquillages de chaque espèce après l'huître est faible (Brien 1990, 309). D'autres sites, Loupian et Balaruc-les-Bains, sont directement sur l'étang de Thau : la masse des huitres, des peignes ou des coquilles Saint-Jacques est énorme, cet apport alimentaire était tel qu'il semblait, peut-être, inutile d'aller prospecter d'autres biotopes pour se nourrir.

\section{$\square$ Conclusions}

Cette étude ponctuelle sur un échantillonnage restreint montre une consommation de deux espèces de coquillages : l'huître surtout et le peigne. Mais la présence d'autres espèces laisse supposer une plus grande variété. Les conditions de la fouille font que les archéologues ont sauvé de la pelle-mécanique en premier lieu les céramiques et ensuite, les coquillages les plus gros. Quelques valves : tapes decussata L. 1758 ou pullastra Montagu 1803, des fragments de mactra glauca Born 1778 et une patella caerulea sont les rares témoins d'un choix beaucoup plus large.

Il est curieux cependant de constater l'absence de la moule mytillus galloprovincialis Lamarck 1799 et du cerastoderma edule L. 1766. Ont-ils échappé aux fouilleurs ou bien n'ont-t-ils figuré qu'en très petite quantité au menu des habitants de NotreDame? Serait-ce l'indice d'un goût alimentaire, d'une coutume laissant les moules et les coques sur les sols, les coquillages plus gros étant stockés ailleurs dans des fosses ? Il se peut aussi que seuls certains coquillages, les huîtres et les peignes, aient été l'objet d'un commerce à l'époque gallo-romaine et dans l'Antiquité tardive.

Tabl. XI Inventaire des espèces de coquillages marins.

\section{Notes de commentaire}

* Laboratoire d'Archéologie Médiévale Méditerranéenne. C.R.A. E.R.A. 6. Université de Provence. 29 av. Robert Schuman 13621 Aix-en-Provence.

* S.A.M. Résidence Beausoleil, Bât. 6-13120 Gardanne.

*** E.R.A. 38. C.R.A., CNRS, Sophia-Antipolis - 06565 Valbonne cédex (et U.R.A. 1415, Paris).

***** Les Cyprès, 82 avenue de la Mascotte - 83140 Six-Fours-les-Plages.

Les contributions des auteurs sont les suivantes : Le mobilier en verre (chap. 3.3.), D. Foy ; La faune terrestre (chap. 5.1.), F. Audoin-Rouzeau ; Les coquillages marins (chap. 5.2.), Fr. Brien-Poitevin.

Crédit des illustrations : cl. S.A.M. (fig. 4, 5 haut); cl. Y. Rigoir (fig. 3, 5 bas, 32, 54, 59, 60, 61) ; cl. A. Chéné, CNRS (fig. 70). 
1 - Renseignement fourni par M. Porte, concierge de l'école.

2 - Ouvrage collectif sous la direction de G. Démians d'Archimbaud, à paraître dans la collection des Doc. Archéol. Franç. ; pour plus de simplicité, les références à ce travail étant nombreuses, nous renverrons dans la bibliographie à : Démians d'Archimbaud et al. à paraître, suivi éventuellement des numéros d'illustratiøns.

3 - Au Laboratoire de céramologie de Lyon (E.R.A. 3 du C.R.A.), par Mauricè Picon.

4 - Banque de données travaillant en collaboration avec le programme H3 du Conseil Supérieur de la Recherche Archéologique :"Mines et métallurgie anciennes".

5 - Parmi les contacts pris pour l'étude de ces creusets, citons : MM. J.-P. Mohen, conservateur du Musée des Antiqutés Nationales de Saint-Germain-en-Laye ; Ph. Andrieux, archéologue départemental du Val-de-Marne ; M. Pernot, du Laboratoire de Recherche des Musées de France (CNRS).

6 - Par Mme E. Rabeisen, conservatrice du musée d'Alise Sainte-Reine.

7 - Authentifiées par M. Mangin et en cours d'analyse.

8 - Documentation fournie par Y. et J. Rigoir.

9 - Analyses en cours au Laboratoire de Céramologie de Lyon.

10 - On choisit ici, pour un mouton compté pour 1 , d'estimer à 7 fois plus important le rendement en viande moyen d'un bœuf et à 2,5 fois celui d'un porc. Les agneaux, veaux et porcelets sont respectivement comptés pour 0,25, 1,75 et 0,6.

11 - Calculs de hauteur au garrot effectués selon les facteurs de Teichert 1975.

12 - Calculs de hauteur au garrot effectués selon les facteurs de Fock 1966 (Driesch, Boessneck 1974).

13 - Calculs de hauteur au garrot effectués selon les facteurs de Kiesewalter 1888.

14 - Calculs de hauteur au garrot effectués selon les facteurs de Koudelka 1885.

15 - Je souhaite remercier V. Eisenmann qui a bien voulu m'apporter son concours pour affiner la détermination des restes d'équidés, J. Pichon qui s'est chargée de l'identification des restes d'oiseaux, et F. Poplin pour les utiles suggestions qu'il m'a apportées.

\section{Renvois bibliographiques}

Alenus-Lecerf 1986 : ALENUS-LECERF (J.) - Le cimetière de Vieuxville : quelques considérations préliminaires. In $: \mathrm{La}$ Civilisation mérovingienne dans le bassin mosan. Actes du colloque international d'Amay-Liège (22-24 août 1985). Liège, éd. Notte et Willems, 1986, pp. 181-193.

Atlante 1981 : Atlante delle forme ceramiche. I- Ceramica fine romana nel bacino del Mediterraneo (medio e tardo impero) (A. Carandini dir.). Roma, 1981, 270 p., 165 pl. (Enciclopedia dell'Arte antica).

Audoin-Rouzeau 1991 : AUDOIN-ROUZEAU (F.) - La taille du bouf domestique de l'Antiquité aux Temps Modernes. Juan-lesPins, éd. C.R.A.-CNRS/A.P.D.C.A., 1991, pp. 3-40 (Fiches d'Ostéologie animale pour l'Archéologie, série B, 2).

Audoin-Rouzeau à paraître : AUDOIN-ROUZEAU (F.) - La taille des animaux d'élevage à l'époque romaine et leur exportation. Actes du Colloque Piganiol (Nantes, 1991). A paraitre dans les Publ. du Centre de Recherches A. Piganiol.

Bayard 1990 : BAYARD (D.) - L'ensemble du grand amphithéâtre de Metz et la sigillée d'Argonne au Ve siècle. Gallia, 47, 1990, pp. 271-319.

Benet 1989 : BENET i ARQUE (C.), SUBIAS i PASCUAL (A. et E.) - Els Vidres. Un abocador del segle V D.C. en el forum provincial de Tarraco. Tarragona, 1989, pp. 329-349 (Memories d'excavacio ; Taller Escola d'Arqueologia, 2).

Benoit 1934 : BENOIT (F.) - Forma Orbis Romani. Carte archéologique de la Gaule Romaine, Bouches-du-Rhône. Paris, 1934, 232 p.

Bérato 1986 : BERATO (J.), BORREANI (P.), LECACHEUR (P.), PASQUALINI (M.), THEVENY (J.-M.), RIGOIR (J. et Y.) Fouilles récentes à Toulon (Var), Quartier Besagne, 1985-1986. Doc. Archéol. Mérid., 9, 1986, pp. 135-166.

Boixadéra 1987 : BOIXADERA (M.), BONIFAY (M.), PELLETIER (J.-P.), RIGOIR (J. et Y.), RIVET (L.) - L'habitat de hauteur de Sainte-Propice (Velaux, B.-du-Rh.). L'occupation de l'Antiquité tardive. Doc. Archéol. Mérid., 10, 1987, pp. 91-113.

Bökönyi 1974 : BÖKÖNYI (S.) - History of Domestic mammals in
Central and Eastern Europe. Budapest, Akademiai Kiado, 1974, $597 \mathrm{p}$.

Bonifay 1983 : BONIFAY (M.) et coll. - Fléments d'évolution des céramiques de l'Antiquité tardive à Marseille d'après les fouilles de la Bourse. $R$. Archéol. Narb., 16, 1983, pp. 285-346.

Bonifay 1986 : BONIFAY (M.) et RIGOIR (Y.) - Les formes de Dérivées-des-Sigillées Paléochrétiennes à bec tubulaire. Doc. Archéol. Mérid., 9, 1986, pp. 216-219.

Boube-Piccot 1975 : BOUBE-PICCOT (C.) - Les bronzes antiques du Maroc. II- Le Mobilier. Et. et trav. d'archéol. marocaine, V, 1975,465 p. et $286 \mathrm{pl}$.

Boucher 1971 : BOUCHER (St.) - Vienne. Bronzes antiques. Inventaire des collections publiques françaises, Paris, éd. Musées Nationaux, 1971, 232 p. et 908 pl.

Boucher 1976 : BOUCHER (St.) et TASSINARI (S.) - Bronzes antiques du Musée de la civilisation gallo-romaine à Lyon. IInscriptions, statuaire, vaisselle. Lyon, Diff. De Boccard, 1976, $155 \mathrm{p}$.

Brien 1988 : BRIEN (Fr.) - Les coquillages. In : Martigues, village gaulois (J. Chausserie Laprée et N. Nin dir.). Dossiers Hist. et Archéol., 128, 1988, pp. 96-97.

Brien 1990 : BRIEN (Fr.) - Etude conchyliologique. In : Le village gallo-romain et médiéval de Lunel Viel (Hérault). La fouille du quartier ouest (1981-1983) (Cl. Raynaud dir.). Paris, éd. les Belles Lettres, 1990, 353 p. (A. Litt. Univ. Besançon, 422).

Brien à paraître : BRIEN (Fr.) - Etude conchyliologique. In : L'oppidum de Saint-Blaise (commune de Saint-Mitre-les-Remparts, Bouches-du-Rhône). La réoccupation aux $V^{e}-V_{I I}{ }^{c} s$. d'après les fouilles récentes (G. Démians d'Archimbaud dir.). A paraître dans les Doc. d'Archéol. Franç.

Brisou 1985 : BRISOU (J.) - Les coquillages dans l'histoire des hommes. Toulouse, éd. Ouest-France, 1985, $140 \mathrm{p}$.

Bucquoy 1886 : BUCQUOY (E.), DAUTZENBERG (Ph.) et DOLFUS (G.) - Les mollusques marins du Roussillon (2 vol.). Paris, éd. Baillière, 1882-1886, 1455 p., 165 pl. 
Buxeda à paraître : BUXEDA (J.), CAU (M.-A.) et TUSSET (F.) Appréciation macroscopique et détermination des fabriques. In : Actes du Congrès de Cognac 1991, S.F.E.C.A.G. (à paraître).

Cat. expo 1987 : Autun-Augustodunum. Capitale des Eduens. Catalogue d'exposition (Musée Rolin, 16 mars/27 octobre 1985). Autun, 1987, $411 \mathrm{p}$.

C.A.T.H.M.A. 1986 : Association C.A.T.H.M.A - La céramique du Haut Moyen Age en France méridionale : éléments comparatifs et essai d'interprétation. In : La ceramica medievale nel mediterraneo occidentale, $3^{\mathrm{e}}$ colloque international (Sienne, 1984). Florence, 1986, pp. 27-50.

C.A.T.H.M.A. 1989 : Association C.A.T.H.M.A - Réunion du 15 décembre 1989. Compte-rendu ronéotypé.

C.A.T.H.M.A. 1990 : Association C.A.T.H.M.A - Quantification et chronologie : quelques applications sur les contextes de l'Antiquité tardive et du Haut Moyen Age dans le midi de la Gaule : méthodes, intérêts et limites. In: Actes du Congrès de Mandeure-Mathay, S.F.E.C.A.G., 1990, pp. 149-159.

C.A.T.H.M.A. à paraître : Association C.A.T.H.M.A - Importations de céramiques communes méditerranéennes dans le midi de la Gaule ( $\mathrm{V}^{\mathrm{c}}$-VII ${ }^{\mathrm{c}} \mathrm{s}$.). In : La ceramica medieval do mediterraneo occidental, $4^{\mathrm{c}}$ colloque international, Lisbonne, 1987 (à paraître).

Cavaillès-Llopis 1986 : CAVAILLES-LLOPIS (M.-T.) - Céramiques de l'Antiquité tardive à Marseille (sondages D II 11 et D II 15). Doc. Archéol. Mérid., 9, 1986, pp. 167-195.

Chaillan 1910 : CHAILLAN (M.) - Recherches archéologiques et historiques sur Gardanne. Paris, éd. Picard, 1910, 78 p.

Codou 1989 : CODOU (Y.) - Saint-Pantaléon (Vaucluse). In : L'église, le terroir (M. Fixot et E. Zadora-Rio dir.). Paris, éd. du CNRS, 1989, pp. 146-149 (Monographie du C.R.A., 1).

Codou 1991 : CODOU (Y.) - L'église de Saint-Pantaléon (Vaucluse). Approche des églises à deux nefs. In : Histoire et archéologic religieuse en pays d'Apt. Actes du II' Colloque d'Apt (1983). Apt, éd. Archipal, 1991, pp. 83-97.

Columeau 1989 : COLUMEAU (Ph.) - Les vertébrés. In : L'oppidum d'Ambrussum et son territoire (J.-L. Fiches dir.). Paris, éd. du CNRS, 1989, pp. 209-218 (Monographic du C.R.A., 2).

Congès 1983 : CONGES (G.), BONIFAY (M.), BRUN (J.-P.) et PASQUALINI (M.) - Un dépotoir de la fin de l'Antiquité dans la grotte de la Fourbine, Saint-Martin-de-Crau (Bouches-duRhône). R. Archéol. Narb., 16, 1983, pp. 347-364.

Czurda-Ruth 1989 : CZURDA-RUTH (C.) - Zu den römischen Gläsern aus den Hanghäusern von Ephesus. Glas der Caesaren, Römisches Glas des 2-bis 6. Jahrhunderts der Archäologische Befund (Berichte auf dem Glassymposium in Köln, 1988). Köln, 1989, pp. 129-140 (Kölner Jahrbuch für Vor-und Frühgeschichte, 22).

Dasnoy 1956 : DASNOY (A.) - Coupes en verre ornées de symboles chrétiens. A. Soc. archéol. de Namur, 48, 1956, pp. 360-373.

Démians d'Archimbaud 1980 : DEMIANS D'ARCHIMBAUD (G.) - Les fouilles de Rougiers. Contribution à l'archéologie de l'habitat rural médiéval en pays méditerranéen. Paris, éd. du CNRS, 1980 (1982), 724 p.

Démians d'Archimbaud et al. à paraître : DEMIANS D'ARCHIMBAUD (G.) (dir.) (avec notamment la participation de D. Foy pour l'étude du verre, de J-P. Pelletier, Y. et J. Rigoir et $L$. Vallauri pour l'étude des céramiques) - L'oppidum de Saint-Blaise (commune de Saint-Mitre-les-Remparts, Bouchesdu-Rhône). La réoccupation aux $V^{c}-V_{1}{ }^{c} s$., d'après les fouilles récentes. A paraître dans les Doc. Archéol. Franç.

Derville, Poplin 1977 : DERVILLE (M.) et POPLIN (F.) - La faune. In : Deux puits gallo-romains à Levroux (Indre) (O. Buschenschutz et A. Ferdière). R. A. E., 16, 1-2, 1977, pp. 45-48.

Desbat 1986 : DESBAT (A.) et PICON (M.) - Sigillée claire B et "luisante" : classification et provenance. Figlina, 7, 1986, pp. 5-18.
Docquier 1984 : DOCQUIER (J.), STRAUS (J.-A.), THIRION (E.) et WILLEMS (J.) - Huy au temps de la christianisation et des mérovingiens. Catalogue d'exposition, Huy, 1984.

Donati 1981 : DONATI (P.) - Il campanato. Quaderni d'informazione, 8, 1981.

Driesch, Boessneck 1974 : DRIESCH (A. Van den) et BOESSNECK (J.) - Kritische Amerkungen zur Widerristhöhenberechnung aus Längenmaßen vor-und frühgeschichtlicher Tiervenochen. Münich, 1974, pp. 325-348 (Säugetierkundliche Mitteilungen, 22).

Eisenmann 1986 : EISENMANN (V.) - Comparative Osteology of Modern and Fossil Horses, Half-Asses, and Asses. In : Equids in the Ancient World (Reihe A.). Wiesbaden, éd. Meadow and Uerpmann, 1986, pp. 67-116 (Beihefte zum Tübingen Atlas des vorderen orients NR 19/1).

Eisenmann, Beckouche 1986 : EISENMANN (V.) et BECKOUCHE (S.) - Identification and Discrimination of Métapodials from Pleistocène and Modern Equus, Wild and Domestic. In : Equids in the Ancient World (Reihe A.). Wiesbaden, éd. Meadow and Uerpmann, pp. 117-163 (Beihefte zum Tübingen $\Lambda$ tlas des vorderen orients NR 19/1).

Faider-Feytmans 1940 : FAIDER-FEYTMANS (G.) - Les verreries des époques romaine et mérovingienne au Musée de Mariemont. $R$. belge archéol. et hist. de l'art, 10, 1940, pp. 211-289.

Faider-Feytmans 1970 : FAIDER-DEYTMANS (G.) - Les collections d'archéologie régionale du musée de Mariemont. II- Les nécropoles mérovingiennes. Morlanwelz-Mariemont, éd. Musée de Mariemont, 1970 (2 vol.), 270 p. et 151 pl.

Foy 1977 : FOY (D.) - Verreries paléochrétiennes découvertes récemment à Marseille. $R$. Archéol. Narb., 10, 1977, pp. 273-286.

Foy 1984 : FOY (D.) et BONIFAY (M.) - Eléments d'évolution des verreries de l'Antiquité tardive à Marseille d'après les fouilles de la Bourse (1980). R. Archéol. Narb., 17, 1984, pp. 289-308.

Foy 1990 : FOY (D.) (avec la collaboration de J.-B. Féraud, L.-Fr. Gantès, M. Moliner, M. Picon) - Creusets de verrier de l'Antiquité tardive et du haut Moyen Age découverts dans le Sud-Est de la France. In: Annales du $11^{\circ}$ Congrès de l'Association Internationale pour l'Histoire du verre (Bâle 1988). Liège, 1990, pp. 199-216.

Foy 1991 : FOY (D.) - Les verres. In : La basilique paléochrétienne du Clos de La Lombarde à Narbonne. Cadre archéologique, vestiges et mobilier (Y. Solier dir.). Paris, éd. du CNRS, 1991, pp. 255-271 (Suppl. à la R. Archéol. Narb., 23).

Foy à paraître : FOY (D.) - Les verres. In : L'oppidum de SaintBlaise (commune de Saint-Mitre-les-Remparts, Bouches-duRhône). La réoccupation aux $\mathrm{V}^{\mathrm{c}}-\mathrm{VII}^{\mathrm{c}} \mathrm{s}$. d'après les fouilles récentes (G. Démians d'Archimbaud dir.). A paraître dans les Doc. Archéol. Franç.

Gandolfi 1981 : GANDOLFI (D.) - Terre sigillata chiara D di Albintimilium. R. Et. Lig., 47, 1981 (Homm. à N. Lamboglia), pp. 53-149.

Gandolfi 1986 : GANDOLFI (D.) - La pietra ollare a Ventimiglia. R. Et. Lig., 52, 1986, pp. 269-302.

Granier 1984 : GRANIER (J.) et PENEZ (A.) - Catalogue des mollusques marins et saumâtres observés, récoltés ou signalés sur le littoral de la Camargue et de la partie orientale du Golfe d'Aigues-Mortes. B. Soc. Et. Sci. Nat. Vaucluse, 1982-1984, pp. 51-164.

Guérard 1857 : GUERARD (M.) - Cartulaire de l'abbaye de SaintVictor de Marseille. Paris, 1857.

Guéry 1972 : GUERY (R.) - Nouveaux fragments de plats rectangulaires en terre cuite. BABesch, XLII, 1972, pp. 114-124.

Guéry 1990 : GUERY (R.) - A propos de quelques fragments de plats rectangulaires en terre cuite découverts en Narbonnaise. Doc. Archéol. Mérid., 13, 1990, pp. 266-271.

Guild 1980: GUILD (R.), GUYON (J.) et RIVET (L.) - Recherches archéologiques dans le cloître Saint-Sauveur d'Aix-en-Provence. 
Bilan de quatre campagnes de fouilles (1976-1979). R. Archéol. Narb., XIII, 1980, pp. 115-164.

Guild 1983 : GUILD (R.), GUYON (J.) et RIVET (L.) - Les origines du baptistère de la cathédrale Saint-Sauveur. Etude de topographie aixoise. R. Archéol. Narb., XVI, 1983, pp. 171-232.

Graillot 1899 : GRAILLOT (M.) - Objets d'archéologie chrétienne trouvés à Autun. M. Soc. Eduenne, XXVII, 1899, pp. 46-56.

Grosso-Paglieri 1986 : GROSSO-PAGLIERI (G.) et PAOLI-MAINERI (M.-C.) - Observazioni sulla pietra ollare trovata ad Albenga. R. Et. Lig., 52, 1986, pp. 253-268.

Harden 1936 : HARDEN (D.B.) - Roman glass from Karanis found by the University of Michigan, Archaeological expedition in Egypt (1924-29). 1936 (Univ. of Michigan Studies Humanistic Series, XLI).

Hayes 1972 : HAYES (J.W.) - Late Roman Pottery. Londres, The British School at Rome, 1972, 477 p.

Hayes 1976 : HAYES (J.W.) - Pottery Report. In : Excavation at Carthage 1975 conducted by the University of Michigan (J.H. Humphrey éd., vol. IV). Tunis, éd. Ann Arbor, 1976.

Hayes 1980 : HAYES (J.W.) - Problèmes de la céramique des VII ${ }^{c}$ $\mathrm{IX}^{\mathrm{c}} \mathrm{s}$. à Salamine et à Chypre. In : Salamine de Chypre, Histoire et archéologie. Paris, éd. du CNRS, 1980, pp. 375-380 (Colloques internationaux du CNRS).

Ising 1957 : ISING (C.) - Roman Glass from dated finds, archeologica Traiectina, Il. Groningen- Djakarta, 1957.

Jessup 1946 : JESSUP (R.F.) - An Anglo-saxon cemetery at Westbere, Kent. The Antiquaries Journal, 26, 1946, pp. 11-21.

Johansson 1987 : JOHANSSON (F.) - Zoologische und kulturgeschichtliche Untersuchungen an den Tierresten aus der römischen Palastvilla in Bad Kreuznach. Kiel, 1987, 180 p. (Schriften aus der Archäologisch-Zoologischen Arbeits-gruppe SchleswigKiel, 11).

Jourdan 1976 : JOURDAN (L.) - La faune du site gallo-romain et paléochrétien de La Bourse (Marseille). Paris, éd. du CNRS, 1976, 338 p.

Kauffmann 1987 : KAUFFMANN (A.), PELLETIER (J.-P.) et RIGOIR (J. et Y.) - Les céramiques de l'Antiquité tardive au $\mathrm{XI}^{\mathrm{c}}$ s. dans les fouilles de la place Jean Jaurès à Apt (Vaucluse), premières études. Archéol. Midi Médiév., 5, 1987, pp. 61-84.

Kiesewalter 1888 : KIESEWALTER (L.) - Skelettmessungen an Pferden als Beitrag zur theoretischen Grundlage der Beurteilungslehre des Pferdes. Leipzig, Diss, 1888.

Koudelka 1885 : KOUDELKA (F.) - Das Verhältnis der Ossa longa zur Skeletthöhe bei den Säugetieren. Verhandl. d. Natforsh. Ver. in Brünn, 24, 1885, pp. 127-153.

Lamboglia 1950 : LAMBOGLIA (N.) - Gli scavi di Albintimilium e la cronologia della ceramica romana. I- Campagne di scavo 19381940. Bordighera, Ist. Intern. di St. Lig., 1950, 200 p., 6 pl. (Coll. di Monogr. preist. ed archeol., II).

Legoux 1978 : LEGOUX (Y. et R.) - Verrerie, vaisselle de bronze et céramique de la nécropole mérovingienne de Sainte-Fontaine à Bulles (Oise). Cab. Archéol. Picardie, 5, 1978, pp. 231-240.

Lipper 1981-82 : LIPPER (E.) - Die Tierknochenfunde aus dem römischen Kastell Abusina-Eining, Stadt Neustadt A. D. Donau, Lkr. Kelheim, 1981-82, 160 p. (Bericht der Bayerischen Bodendenkmalpflege, 22/23).

Luff 1982 : LUFF (R.-M.) - A zooarchaeological study of the roman north-western provinces. Oxford, 1982, 338 p. (BAR International Series, 137).

Marco 1989 : MARCO (V.) et MOCCI (Fl.) - Histoire de l'habitat et occupation du sol dans la moyenne vallée de l'Arc. Carte archéologique du canton de Gardanne. Mém. de maîtrise, Univ. de Provence, 1989, 166 et 196 p. (dactyl.).

Motteau 1985 : MOTTEAU (J.) - Aspects de la vaisselle de verre médiévale. Recherches sur Tours, 4, 1985, pp. 9-38..
Murialdo 1986 : MURIALDO (G.), FOSSATI (A.), FALCETTI (C.) et BONORA (E.) - La pietra ollare nel Finale. $R$. Et. Lig., 52, 1986, pp. 217-242.

Olcese 1989 : OLCESE (G.) - La ceramica comune di Albintimilium : notizie preliminari sull'indagine archeologica e archeometrica. R. St. Lig., 1-4, 1989, pp. 149-228.

Parenzan 1976 : PARENZAN (P.) - Carta d'identita delle conchiglie del mediterraneo. Tarente, éd. Bios Taras (3 vol.), 1970-1976, $543 \mathrm{p} ., 53 \mathrm{pl}$.

Paunier 1987 : PAUNIER (D.) - La pierre ollaire dans l'Antiquité en Suisse orientale. In : La Pietra ollare della preistoria all'età moderna, Atti del convegno (Como, 1982). Como, Archeologia dell'Italia settentrionale, 5, 1987, pp. 47-57.

Pelletier 1983 : PELLETIER (J.-P.) - La céramique commune à la pâte grise. In : Les origines du baptistère de la cathédrale SaintSauveur. Etude de topographie aixoise (R. Guild, J. Guyon et L. Rivet). R. Archéol. Narb., 16, 1983, pp. 214-216.

Pelletier 1984 : PELLETIER (J.-P.) - La céramique commune à pâte grise, étude préliminaire. In : L'établissement gallo-romain de Saint-Julien-les-Martigues (L. Rivet). Rapport de fouille dactylographié, 1984, pp. 179-185.

Perin 1972 : PERIN (P.) - Deux verreries exceptionnelles provenant de la nécropole mérovingienne de Mézières, la corne à boire de la tombe $\mathrm{n}^{\circ} 74$ et la coupe à décor chrétien de la tombe $\mathrm{n}^{\circ} 89$. Journal of Glass Studies, XIV, 1972, pp. 67-76.

Pfeifer 1986 : PFEIFER (H.-R.) et SERNEELS (V.) - Inventaire des gisements de pierre ollaire au Tessin et dans les régions voisines : aspects minéralogiques et miniers. In : 2000 anni di pietra ollare. Quad. d'inf., 11, 1986, pp. 147-235.

Poulain-Josien 1964 : POULAIN-JOSIEN (Th.) - Les animaux domestiques et sauvages en France du Néolithique au Galloromain. Etude d'ethnozoologie à partir de vestiges osseux ; équidés, suidés, bovidés. Thèse de III ${ }^{c}$ cycle, Univ. de Paris, 1964, 351 p. et 8 fig.

Poulain-Josien 1966a : POULAIN-JOSIEN (Th.) - Etude de la faune. In : Un dépotoir augustéen à Argentomagus (Saint-Marcel, Indre) (Th. Poulain-Josien, J. Allain, A. Cotheney, M. Vauthey). R.A.C., V, 1, 1966, pp. 17-39

Poulain-Josien 1966b : POULAIN-JOSIEN (Th.) - La faune. In : Un puits funéraire de la fin du $\mathrm{I}^{\mathrm{er}} \mathrm{s}$. aux Aquae Siccae (Cazères, Haute-Garonne) (G. Manière). Gallia, 24, 1966, pp. 147-159.

Poulain-Josien 1972 : POULAIN-JOSIEN (Th.) - La faune des grands mammifères des couches paléochrétiennes de la grotte de l'Hortus (Valflaunès, Hérault). Et. Quat., 1972, pp. 209-228 (Mémoire, 1).

La pietra ollare... 1986 : La pietra ollare in Liguria. Atti della giornata di studi in ricordo di Lella Massari (Finale Ligure, 22 giugno 1985). R. Et. Lig., 52, 1986, pp. 151-319.

La pietra ollare... 1987 : La pietra ollare della preistoria all'età moderna. Atti del convegno (Como 16-17 ottobre 1982), Museo Civico Archeologico "Giovio". Como, éd. New Press, 1987 (Archeologia dell' Italia Settentrionale, 5).

Queixalos 1987 : QUEIXALOS (I.), MENU (M.) et MOHEN (J.-P.) - Creusets pour la fonte des alliages à base de cuivre du Bronze final au Fort Harrouard à Sorel-Moussel (Eure-etLoire). B. Soc. Prébist. Franç., 84, 1, 1987, pp. 23-30.

Raynaud 1990 : RAYNAUD (Cl.) - Le village gallo-romain de Lunel Viel (Hérault). Paris, les Belles Lettres, 1990, 353 p. (A. Litt. Univ. Besançon, 422).

Rigoir 1985 : RIGOIR (Y. et J.) et RIVET (L.) - Les Dérivées-desSigillées Paléochrétiennes. Exportations et influences entre le groupe provençal et le groupe languedocien. Doc. Archéol. Mérid. 8, 1985, pp. 87-99.

Rigoir 1987 : RIGOIR (Y. et J.) et RIVET (L.) - Cruches et pots en Sigillée Paléochrétienne. In : Actes du Congrès de Caen. S.F.E.C.A.G., 1987, pp. 183-206. 
Salomonson 1969 : SALOMONSON (S.-W.) - Spätrömische rote Tonware mit Reliefverzierung aus nordafrikanischer Werkstätten. BABesch, 44, 1969, pp. 4-109.

Statistique 1824 : VILLENEUVE (Comte de) - Statistique du département des Bouches-du-Rhône, II. 1824. Marseille, éd. A. Ricard, 1824, $1212 \mathrm{p}$.

Sternini 1989 : STERNINI (M.) - Una manifattura vetraria di V secolo a Roma. Quad. del dipart. di archeol. e st. delle arti, sez. archeol., 1989.

Tassinari 1975 : TASSINARI (S.) - La vaisselle de bronze romaine et provinciale au Musée des Antiquités Nationales. Paris, éd. du C.N.R.S., 1975 (Suppl. à Gallia, 29).

Tatton-Brown 1984 : TATTON-BROWN (V.-A.) - In : The glass in Excavations at Carthage : the British mission. Vol. I, 1. The Avenue du Président Habib Bourguiba, Salammbo : the site and finds other than pottery (H.R. Hurst et S.P. Roskams). British Acad. from the Univ. of Sheffield, Depart. of Prehist. and Archaeol., 1984, pp. 194-212.

Teichert 1975 : TEICHERT (M.) - Osteometrische Untersuchungen zur Berechnung der Widerristhöhe bei Schafen. Amsterdam, éd.

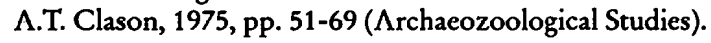

Thiriot 1986 : THIRIOT (J.) - La production de la céramique com- mune grise du Haut Moyen Age en Uzège et Bas-Rhône : état de la question. In : La ceramica medievale nel mediterraneo occidentale, $3^{\mathrm{c}}$ colloque international (Sienne, 1984). Florence, éd. All'insegna del Giglio, 1986, pp. 235-250.

Tylecote 1962 : TYLECOTE (R.F.) - Metallurgy in Archaology. Londres, 1962 (sp. pp. 130-141).

Tylecote 1976 : TYLECOTE (R.F.) - History of metallurgy. Londres, 1976, (sp. pp. 16-19).

Vadet 1979 : VADET (A.) - Les ossements animaux. In : Le site gallo-romain des "Terres noires" à Lewarde (Nord) (P. Demolon, M. Tuffreau-Libre et A. Vadet). R. du Nord, 61, 243, 1979, pp. 898-916.

Vigne 1989 : VIGNE (J.-D.) - Les mammifères post-glaciaires de Corse, étude archéozoologique. Paris, éd. du CNRS, 1989, 337 p., 1 pl. microfiches (Suppl. à Gallia-Prébist., 26).

Webster 1980 : WEBSTER (L.), HARDEN (D.) et HASSAL (M.) The Darenth Park Bowl. The Antiquaries Journal, LX, 1980, part. II, pp. 338-341.

Werner 1956 : WERNER (J.) - Les écuelles de verre soufflé en moule. In : Le cimetière franc de Maillot (J. Brever et $\mathrm{M}$. Roosens). A. Soc. Archéol. Namur, 48, 2, 1956, pp. 307-311. 